\title{
Alternate Modal Combination Methods in Response Spectrum Analysis
}

Manuscript Completed: September 1990

Date Published: October 1990

Prepared by

P. Bezler, J. R. Curreri, Y. K. Wang, A. K. Gupta

Brookhaven Irational Laboratory

Upton, NY 11973

Prepared for

Division of Engineering

Office of Nuclear Regulatory Research

U.S. Nuclear Regulatory Commission

Washington, DC 20555

NRC FIN A3955

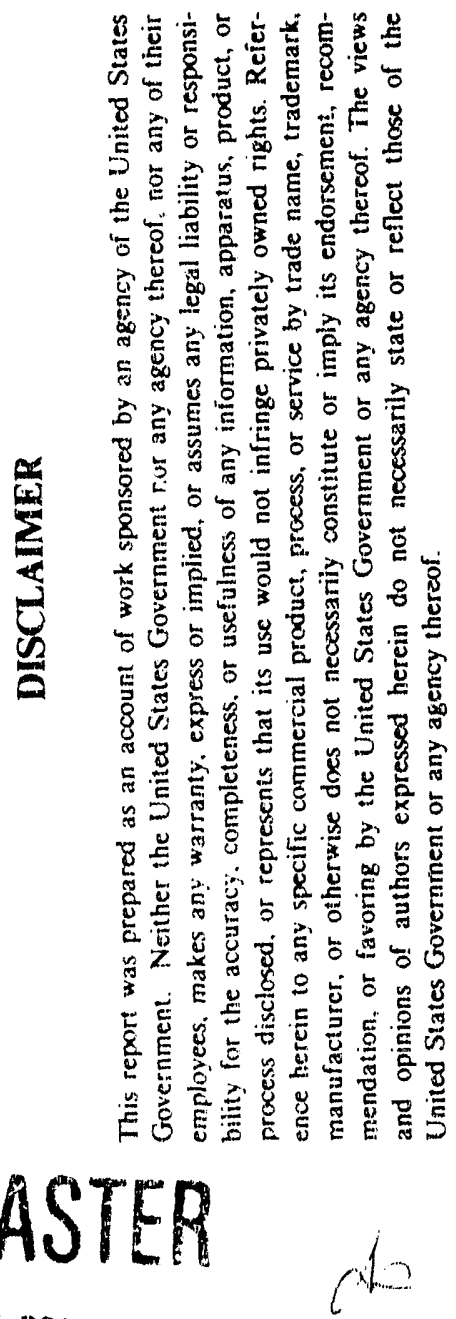




\section{ABSTRACT}

In piping analyses using the response spectrum method square Root of the sum of the squares (SRSS) with clustering between closely spaced modes is the combination procedure most commonly used to combine between the modal response components. This procedure is simple to apply and normally yields conservative estimates of the time history results. The purpose of this study is to investigate alternate methods to combine between the modal response components. These methods are mathematically based to properly account for the combination between rigid and flexible modal responses as well as closely spaced modes. The methods are those advanced by Gupta, Hadjian and Lindley-Yow to address rigid response modes and the Double sum Combination (DSC) method and the complete Quadratic Combination (CQC) method to account for closely spaced noc's. A direct comparison between these methods as well as the SRSS procedure is made by using them to predict the response of six piping systems. The results provided by each method are compared to the corresponding time history estimates of results as well as to each other. The degree of conservatism associated with each method is characterized. 


\section{EXECUTIVE SUMMARY}

The response spectrum method is most commonly used to estimate the seismic response of piping systems. In this method a response quantity is computed for each mode, each direction of excitation and each excitation input. The total response is then formed by summing over all modes, inputs and directions of excitation. Since in this method all time phasing information between response components is lost, no "exact" way to combine the response components to form the total response exists.

To assure acceptable design, the Nuclear Regulatory Commission (NRC) has defined explicit rules to perform these combinations. Specifically the NRC accepts square-root-sum-of-the-squares (SRSS) combination, corrected to account for closely spaced modes, for the summation of modal response components and SRSS combination for the summation of the directional components. However, although it has procedures extant, the NRC recognizes the need to evaluate new and alternate methods and, in this study, has supported the evaluation of modal combination methods that address frequency dependent correlation effects and methods purported to more correctly treat closely spaced modes.

Three methods to address frequency dependent correlation effects and three methods to treat closely spaced modes are evaluated. For frequency effects, the methoc.s advanced by A.K. Gupta, A.H. Hadjian and D.W. Lindley-J.R. Yow are considered. For closely spaced modes, the Rosenblueth Double sum (DSC) method, the complete Quadratic (CQC) method and the SRSS ( $i . e .$, no correction for close spacing) are considered. Additionally, for comparison, an estimate of response based on the NRC accepted procedure is made.

The adequacy or impact of these alternate methods are established by the comparison of the estimates of response developed using these methods to time history estimates of the same response quantities, with the time history estimates being accepted as the true response. Response estimates were made for six different piping systems. Two of these systems were the RHR and AFW systems from zion Niclear Power Plant, and for these, thirty three different seismic excitation records yielding thirty three sets of response estimates were developed. These data give a statistic basis to the study. The remaining four problems ranged from simple to complex, involved the consideration of only one seismic excitation each and were used to round out the problem set.

All evaluations were performed at BNI. In all evaluations only uniform support excitation in the $x$ coordinate direction and a cut of frequency approximation of $100 \mathrm{~Hz}$ is used. For the time history evaluations, the modal superposition method is used. For the response spectrum solutions, the modal method is used and the 
excitation spectra are the unbroadened spectra derived from the corresponding excitation time history records for the same level of damping.

The study results are summarized in both tabular and figure format. They show that all of the methods provide estimates of response that range from small underestimates to large overestimates of the time history response. Regarding the methods to address frequency correlation effects, the response estimates deveioped using these methods provide results that exhibit smaller mean error and dispersion than the method currently accepted by NRC. Regarding the methods to treat closely spaced modes, the study results were inconclusive. It is concluded that the use of either the Gupta, Hadjian or Lindley-Yow methods to address frequency correlation effects in conjunction with either the DSC or $C Q C$ methods to treat closely spaced modes are desirable alternates to the methods currently accepted by the NRC and their use is recommended. Application of these methods will provide estimates of response that closely approach the time history response and never exhibit the gross conservatism typically evident with application of the accepted procedure. 
ABSTRACT. ................................

EXECUTIVE $\operatorname{SUMMARX} \ldots \ldots \ldots \ldots \ldots \ldots \ldots \ldots \ldots \ldots \ldots \ldots \ldots \ldots$

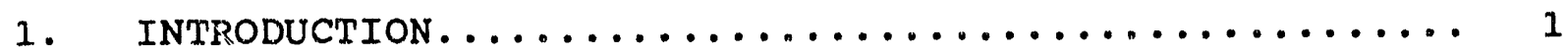

2. DESCRIPTION OF CANDIDATE METHODS............... 3

2.1 Frequency Dependent correlation Effects........ 3

2.1 .1 Gupta Method.................... 4

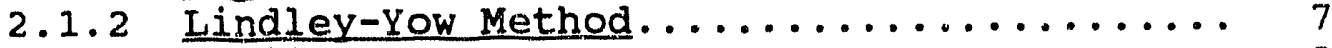

2.1 .3 Hadjian Method.................... 8

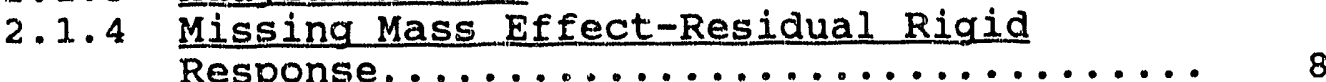

2.2 Closely-spaced Modes..................... 9

2.2.1 Square-Root-Sum-of-Squares (SRSS) Method.. 10

2.2 .2 Rosenblueth Double sum Combination (DSC)

2.2 .3 Complete Quadratic Combination (CQC)

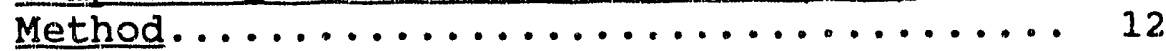

3. PROBLEM SET DESCRIPTION.................... 13

4. STUDY DESCRIPTION......................... 21

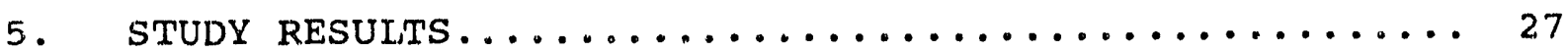

5.1 RHR and AFW - Degree of Exceedance Results...... 29

5.2 BNL Problems - Degree of Exceedance Results..... 29

6. DISCUSSION OF RESULTS .................... 45

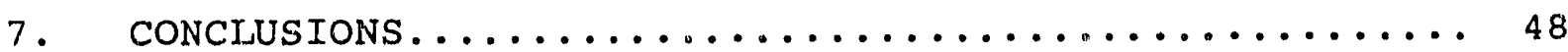

8. REFERENCES........................... 50

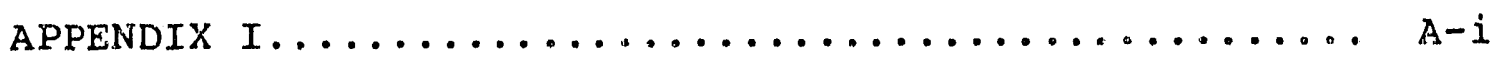

ARPENDIX II ......................... AA-

vii 


\section{LIST OF FIGURES}

\section{FIGURE}

PAGE

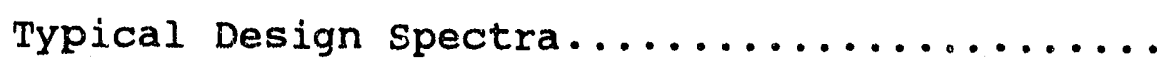

RHR Piping Model...................

AFW Piping Model..................... 15

$\mathrm{z}$ Bend Finite Element Model............. 17

$\mathrm{BM} 1$ and $\mathrm{BM} 2$ Piping Model............... 18

6

BM3 Piping Model...................... 19

7

Input Excitation Records, RHR Problem........

Input Excitation Records, AFW Problem........

Input Excitation Records, $z$ Bend and BMI

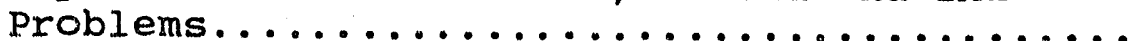

Input Excitation Records, BM2 and BM3 Problems RHR Problem statistical Results...........

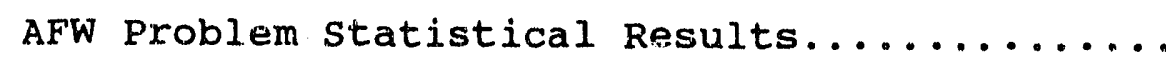

$\mathrm{Z}$ Bend Problem Distribution of Results.......

BMI Problem Distribution of Results.......... 


\section{LIST OF TABLES}

\section{TABLES}

PAGE

Model Parameters..................... 20

2

Representative Table of Results...........

RHR Support Force Mean Value Degree of

Exceedance Results...................

3b RHR Support Force stan. Dev. Degree of

Exceedance Results...................

RHR Pipe End Moment Mean Value Degree of

Exceedance Results...................

3d RHR Pipe End Moment Stan. Dev. Degree of

Exceedance Results....................

AFW Support Force Mean Value Degree of

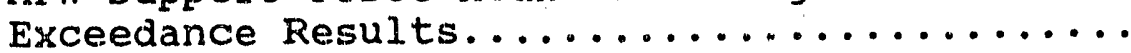

4b AFW Support Force stand. Dev. Degree of

Exceedance Results...................

4c AFW Pipe End Moment Mean Value Degree of Exceedance Results...................

4d AFW Pipe End Moment stand. Dev. Degree of Exceedance Results................... 
The dynamic response of piping systems is most commonly estimated using the response spectrum method. In this method a response quantity is computed for each mode, each direction of excitation and each input, if multiple inputs are considered. The total response is then formed by summing over all modes, inputs and directions of excitation using combination rules designed to provide estimates of system response suitable for design qualification. Although the United states Nuclear Regulatory Commission (USNRC) has defined explicit rules to perform these combinations, new and alternate combination methods, which potentially provide better estimates of system response, have been advanced $[6,7,8]$.

In 1984 the USNRC Piping Review Committee completed a comprehensive evaluation and review of the Seismic Design Requirements for Nuclear Power Plant Piping. The committee's endorsements and recommendations to improve the dynamic load design of piping are presented in NUREG-1061 [1]. Among the items recommended for additional study were: an assessment of the Independent support Motion (ISM) method when combined with Code Case N-411 damping, an investigation of methods which address frequency dependent correlation effects between modes and an evaluation of more rigorous methods to address closely spaced modes. The assessment of the ISM method when combined with Code Case N-411 damping was completed by BNL and reported in NUREG/CR-5105 [2]. The investigation of methods to address frequency dependent correlation effects and to accommodate closely spaced modes are the subject of this report.

Specifically, three methods to address frequency dependent correlation effects and three methods to treat closely spaced modes are evaluated in this study. For frequency effects, the methods advanced by A.K. Gupta, A.H. Hadjian and D.W. Lindley - J.R. Yow are considered. For closely spaced modes, the Rosenblueth Double Sum combination (DSC) method, the complete Quadratic combination (CQC) method and the conventional square-root-sum-of-squares (SRSS) method are considered.

The adequacy or impact of these alternate methods are established by the compaxison of response spertrum estimates of response developed using these methods to time history estimates of the same response quantities. It is noted that the response spectrum method is a design method and is not expected to give results that are in total agreement with the time history results. Just as a design response spectrum represents an average of several response spectra based on several ground motions that among themselves exhibit considerable scatter, the response spectrum method results are likely to compare with the corresponding time history results only approximately. However, on the average, the error between the response spectrum and the time history results are likely to be quite small, tending to zero as the number of 
ground motions considered in the analysis are increased. This will be true only when the modal combination methods used in the analysis are rational and applied correctly.

A description of the candidate methods, the study problem set and the study approach are given in sections 2, 3 and 4. A summary description of the study results are given in section 5 with a complete listing of all study results inciuded as Appendix $I$. The discussion of results is provided in section 6 with a summary of the study conclusions presented in section 7 . Appendix II presents the results of a separate investigation which support the study conclusions. 


\section{DESCRIPTION OF CANDIDATE METHODS}

When the response spectrum method is used to compute inertial response, estimates of response quantities are developed for each mode, each direction of excitation and each input, if multiple inputs are considered. The total response is then formed by summing over all modal component, spatial component and excitation component responses. Unfortunately, since in the response spectrum method all time phasing information between response components is lost, no "exact" way to form the sums exists. For this reason the Nuclear Regulatory Commission (NRC) has specified summing procedures which it deems acceptable for the design of nuclear power plant piping. These include square-root-sum-of-the-squares (SRSS) combination over spatial components, SRSS combination, corrected to consider the interaction of closely spaced modes, over modal components and absolute combination over excitation components $[3,4]$. These methods are considered to provide conservative estimates of the response.

To improve the dynamic load design of piping, the NRC Piping Review Committee recommended that investigations be undertaken to evaluate modal combination methods that properly treat frequency dependent correlation effects and the interaction between closely spaced modes. The recommendations are consistent with the recommendations for change presented by R.P. Kennedy in NUREG/CR1161 [5] and recognize that, realistic and technically sound rules to address these issues have become available, the accepted sRSS method does not address the frequency dependent correlation issue and closely spaced modes are addressed in a simplistic and possible overly conservative fashion. This study was performed to examine the recommendations.

\subsection{Frequency Dependent Correlation Effects}

Three methods to address frequency dependent correlation effects have been evaluated. These are methods advanced by A.K. Gupta [6], A.H. Hadjian [7] and D.W. Lindley - J.R. Yow [8].

In each of these methods the modal responses are considered to consist of two components, a rigid component, $R R_{1}$, and a damped periodic component, $R_{1}$, where $i$ is the mode number. All the rigid components are considered to be perfectly correlated with the input ground motion and are thus perfectly correlated with each other. The total rigid response, $R R$, is then appropriately given by the algebraic sum of all the modal components of rigid response. That is:

$$
\mathrm{RR}=\sum_{i=1}^{\mathrm{n}} \mathrm{RR}_{1}
$$

where $n$ is the total number of modes. 
The damped periodic components, on the other hand, are considered to exhibit the variable degree of correlation normally associated with modal responses. Their sum is provided by any of the candidate modal combination rules which properly consider the interaction of modes as a function of frequency. That is the total damped periodic response, RP, is given by:

$$
R P=\left(\sum_{j=1}^{n} \sum_{k=1}^{n} c_{j k} R P_{j} R P_{k}\right)^{3 / 2}
$$

where $C_{j k}$ are modal coupling factors, the expression for which varies with the combination rule. In this study the DSC, CQC and SRSS combination rules are used to form this sum, as described below.

In forming the above sums, it should be realized that in the response spectrum method, the response in mode $i\left(R_{1}\right)$, and its rigid and damped periodic components ( $R R_{1}$ and $\left.R P_{1}\right)$, all have their unique signs, and those signs are retained throughout the analysis. The uniqueness comes from the fact that the participation factor $\gamma_{1}$ is related to the sign of the modal vector $\{\phi\}$. Thus, the product $\gamma_{1}\left\{\phi_{1}\right\}$, to which all responses $R_{1}$ are related, has unique signs. Some computer programs incorrectly put an absolute sign on $\gamma_{1}$.

The three methods evaluated differ in the specific definition of the modal components of rigid resfonse and damped periodic response and in the method used to sum between the total rigid body and total damped periodic responses to form the total response. A description of each method, as implemented in this study, is provided below. A comparison of the methods, with a discussion of the implication of the differences, is provided in the position paper [9] prepared by R.P. Kennedy for the USNRC Fiping Review committee.

\subsubsection{Gupta Method}

In this method [6] the modal responses are considered to be segregated into three categories according to their associated frequencies. These categories or regimes are differentiated by three frequencies:

$$
\begin{aligned}
& f_{1} \quad \text { - a frequency below which a modal response is assumed } \\
& f_{2} \quad-\quad \text { a frequency above which a modal response is assumed } \\
& \text { to be wholly a rigid component }\left(\mathrm{RP}_{1}=0\right) \\
& \mathrm{f}_{\tau} \quad \text { - the frequency at which the spectral acceleration of } \\
& \text { the excitation returns to the zero period acceler- } \\
& \text { ation, } \mathrm{ZPA}\left(\mathrm{S}_{\mathrm{a}}=\mathrm{ZPA}\right) \text {. }
\end{aligned}
$$


Specifically:

$$
\begin{aligned}
& f_{1}=S_{2 \max } /\left(2 \pi S_{v \max }\right) \\
& f_{2}=\left(f_{1}+2 f_{r}\right) / 3
\end{aligned}
$$

where $S_{a_{\max }}$ and $S_{v_{\max }}$ are the maximum spectral acceleration and velocity of the input. These parameters are illustrated in Figure 1 which shows a typical design spectra in tripartite formate. Using these frequencies modal coefficients, $\alpha_{1}$, are defined such that:

$$
\begin{aligned}
& \alpha_{1}=0, f_{1}<f_{1} \\
& \alpha_{1}=\left(\log \left(f_{1} / f_{1}\right)\right) / \log \left(f_{2} / f_{1}\right), f_{1} \leq f_{1} \leq f_{2} \\
& \alpha_{1}=1.0, f_{1}>f_{2}
\end{aligned}
$$

The above equations for the coefficient $\alpha_{1}$ are based on the average values from several earthquake ground motions. The equation of $\alpha_{1}$ between $f_{1}$ and $f_{2}$ is idealized as a straight line on a semi-log chart. Just as the response spectra from several ground motions used to obtain the design spectrum show considerable scatter, the distribution of $\alpha_{1}$ and the values of $f_{1}$ and $f_{2}$ show scatter from earthquake to earthquake. The idealized semi-log straight line is, however, a rational simplification of a complex problem.

When comparing results from a single time history with those from the corresponding response spectrum, it would be more appropriate to calculate the $\alpha_{1}$-values from the time history responses numerically. The values of $\alpha_{1}$-calculated from the above formula will in general not agree with the numerically calculated values for a single time history. However, in the present study, the $\alpha_{1}$-values from the formula were used based on the values of the key frequencies $\left(f_{1}, f_{2}\right)$ estimated from the appropriate equations above. This procedure would give a poorer agreement between the response spectrum and the time history response quantities than would the procedure where the numerically calculated $\alpha_{1}$-valuas are used in the response spectrum modal combination.

The rigid and periodic response components are then given by:

$$
\begin{aligned}
& R R_{1}=R_{1} \alpha_{1} \\
& R P_{1}=R_{1}\left(1-\alpha_{1}{ }^{2}\right)^{1 / 2}
\end{aligned}
$$

where $R_{1}$ is the predicted modal response corresponding to frequenc' $f_{1}$. The total rigid and periodic responses are then:

$$
R R=\sum_{i=1}^{n} R_{1}
$$




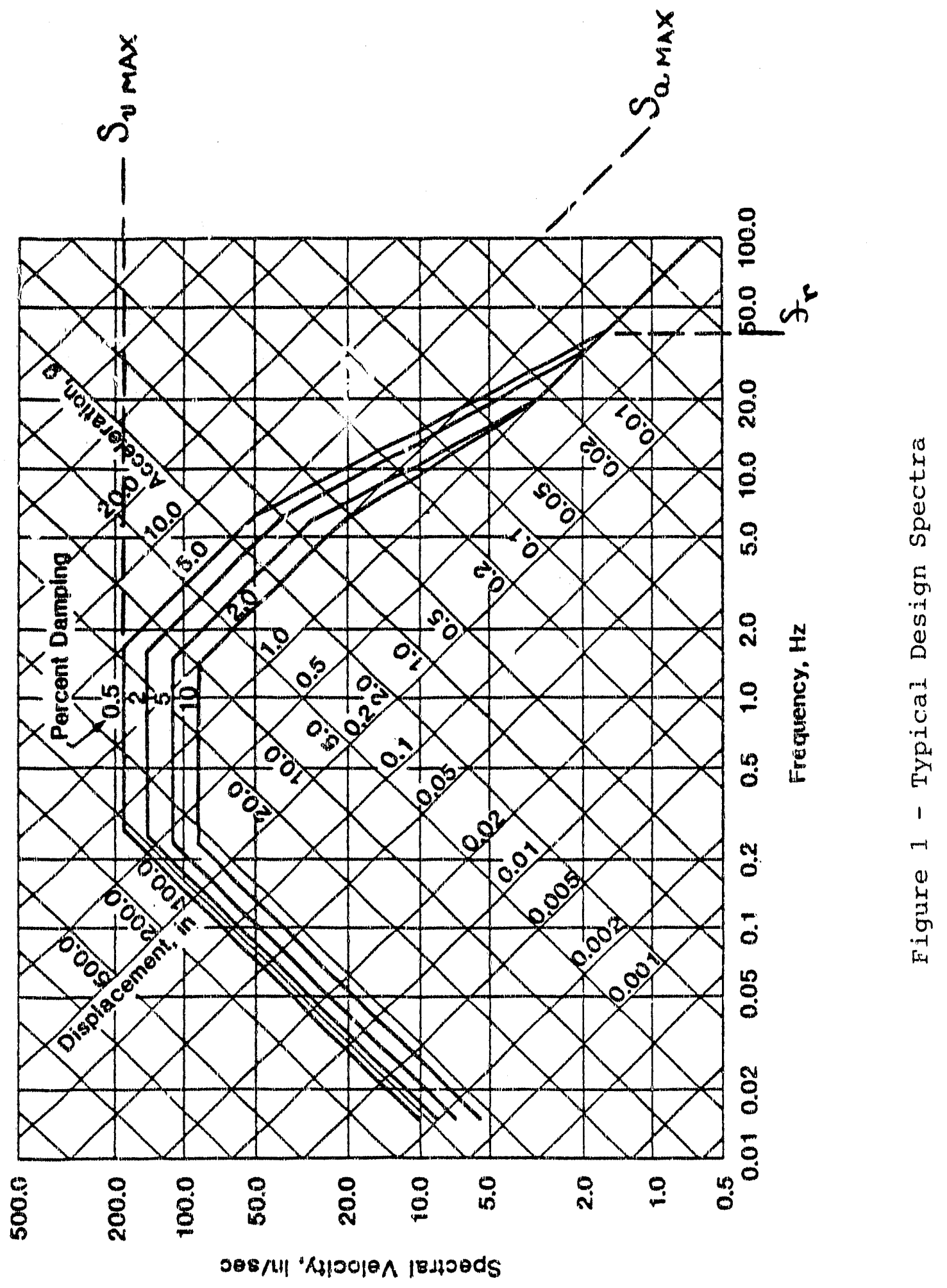




$$
R P=\sum_{j=1}^{n} \sum_{k=1}^{n} c_{j k} R P_{j} R P_{k}
$$

where the $U_{j k}$ are the combination method specific, modal coupling coefficients, as defined in the following sections, and $n$ is the total number of modes being considered. Finally the total response, $R$, is formed by combining the total rigid and periodic responses. In this method these two components of response are considered to be uncorrelated. Therefore:

$$
R=\left(R R^{2}+R P^{2}\right)^{\frac{1}{2}}
$$

\section{1 .2 Iindley-Yow Method}

This method [8] follows the Gupta method except in the definition of the modal coefficients. These are given by:

$$
\alpha_{1}=Z \mathrm{PA} / \mathrm{S}_{\mathrm{al}} \text { and } 0.0 \leq \alpha_{1} \leq 1.0
$$

where $Z P A$ is the zero period acceleration and $S_{a 1}$ is the spectral acceleration for the mude $i$. The modal and total components of rigid and damped periodic response are then given by:

$$
\begin{aligned}
& R R_{1}=R_{1} \alpha_{1} \\
& R P_{1}=R_{i}\left(1-\alpha_{i}{ }^{2}\right)^{\frac{1}{2}} \\
& R R=\sum_{i=1}^{n} R R i \\
& R P=\sum_{j=1}^{n} \sum_{j=1}^{n} c_{j k} R P_{j} R P_{k}
\end{aligned}
$$

In this method the two components are again considerea to be uncorrelated and the total response is therefore:

$$
R=\left(R R^{2}+R P^{2}\right)^{3 / s}
$$

In the low frequency range $\left(f_{1}<2 \mathrm{~Hz}\right)$ the value of $\alpha_{1}$ given by $\mathrm{ZPA} / \mathrm{S}_{\mathrm{al}}$ continues to have a significant value that is physically not rational. If a structural or mechanical systen has significant modes in the low frequency range, a careful evaluation of this method of calculating $\alpha_{i}$ would be desirable. All the problems used in the present study have fundamental frequencies that are greater than $2 \mathrm{~Hz}$ and therefore do rot run into this type of difficulty. 


\subsubsection{Hadjian Method}

This method [7] can also be cast in the samie format as the Gupta method. The definition of the modal coefficients is identical to the definition used in the Lindley-Yow method:

$$
\alpha_{1}=\mathrm{ZPA} / \mathrm{S}_{21} \text { and } 0.0 \leq \alpha_{1} \leq 1.0
$$

With this definition of $\alpha_{1}$ lais method would exhibit the same type of difficulty with problems having significant modes in the low frequency range as the Lindley-Yow method.

The modal and total rigid and periodic response components are respectively:

$$
\begin{aligned}
& R R_{1}=R_{1} \alpha_{1} \\
& R P_{1}=R_{1}\left(1-\alpha_{1}\right) \\
& R R=\sum_{i=1}^{n} R R_{1} \\
& R P=\sum_{j=1}^{n} \sum_{k=1}^{n} C_{j k} R P_{j} R P_{k}
\end{aligned}
$$

The above definition of corresponding rigid and damped periodic modal response components is based on the assumption that these components are additive or in phase. To be consistent with this assumption, the total response is given by:

$$
R=|R R|+|R P|
$$

\section{1 .4 Missing Mass Effect-Residual Rigid Response}

The right hand side of the equation of motion of a multidegree of freedom system is given by $-[M]\left\{U_{b}\right\} \ddot{u}_{b}$, in which $[M]$ is the mass matrix of the system, $\left\{U_{b}\right\}$ is the influence vector representing the static response of the system when subjected to a unit: displacement in the direction of the input motion, and $\ddot{u}_{g}$ is $t$ he input acceleration history. Assuming there are $\mathrm{N}$-total degrees of freedom in the system, there will be $\mathrm{N}$-normal modes $\left\{\phi_{1}\right\}$. The corresponding participation factors are $\gamma_{1}=\left\{\phi_{1}\right\}^{\mathrm{T}}[\mathrm{M}]\left\{U_{\mathrm{b}}\right\}$. The following identity can be easily established [6].

$$
\left\{U_{b}\right\} \sum_{i=1}^{N} \gamma_{L}\left\{\phi_{1}\right\}
$$


The right hand side can thus be written as:

$$
-\sum_{i=1}^{N}[M] \quad \gamma_{1}\left\{\phi_{1}\right\} \quad \ddot{u}_{B}
$$

When only n-modes of vibration are included, $n<N$, the "missing" part of the right hand side can be written as:

$$
-[M]\left\{U_{b}-\sum_{i=n+1}^{N} \gamma_{1} \phi_{1}\right\} \ddot{i}_{g} \text { or }-[M]\left\{U_{b o}\right\} \ddot{u}_{g} \text {. }
$$

The term [M] $\left\{U_{b o}\right\}$ has been called the "missing mass". If all the significant terms of the missirig mass vector are negligibly small as compared to the total mass vector [M] $\left\{U_{b}\right\}$, the missing mass effect would also be negligible and can be ignored. Such is the case in tall buildings and the missing mass effect is not considered.

In many mechanical systems, including piping systems, the missing mass can be significant for practical number of modes $(n$, $n<N)$ included in the analysis. Assuming that the frequency of the last mode included in the analysis is greater than or equal to the rigid frequency $f_{r}$, the missing mass would only generate rigid response. This response is called the "residual rigid response." The relative displacement vector for the residual rigid response is $\left\{U_{0}\right\}=-\{K\}^{-1}[M]\left\{U_{b o}\right\} \ddot{u}_{g}$ in the time history methor or simply $[\mathrm{K}]^{-1}[\mathrm{M}] \quad\left\{\mathrm{U}_{\mathrm{bo}}\right\} \mathrm{ZPA}$ in the response spectrum method. If the corresponding response is $R_{0}$ the above equations for the total rigid response (in all the three methods) should be modified to:

$$
R R=R_{0}+\sum_{i=1}^{n} R R_{1}
$$

Since the concepts of missing mass and the residual rigid responise are well established in the scientific literature [6, 12], and since the effect is common to the three combination methods (Gupta, Hadjian, Lindley-Yow), it was not considered in the present stuay. Both the time history and the response spectrum analyses were performed without considering the residual rigid response. However, in an actual design problem, the residual rigid response must always be considered, and included when significart.

\subsection{Closely-Spaced Modes}

Numerous methods to perform the combiration of peak modal responses are available. The methods deemed acceptable tc the NRC are described in Regulatory Guide 1.92 [4]. These are the Grouping Method, the Ten Percent Method and the Double sum Method. The Grouping and Ten Percent Methods are typically used by industry and 
these rules are essentially sRS combination corrected with absolute combination between modes with associated natural frequencies spaced within 10 percent of each other. These are simple rules that account for the weak correlation cr coupling between modes that have widely different natural freruencies, widely spaced mcides, and the strong correlation or coupling letween closely spaced modes. The Double sum Method is a more sophisticated method that is a conservative variant of the Rosenblueth Double Sum Combination Method considered in this study.

other methods that are available are more sophisticated and are founded in random vibration theory. These methods, although more complex to apply, are considered to more correctly define the coupling between modes. A concise description and comparison of available methods is presented in the above referenced position paper prepared by R.P. Kennedy for the USNRC.

In this study, the combination between peak modal responses is performed $u=i n g$ three alternate combination rules. These include SRSS combination with no correction for closely spaced modes, the Rosenblueth Double Sum combination rule and the compiete Quadratic Combination rule. A brief description of each of these methods is provided below.

\section{2 .1 Square-Root-Sum-of-Squares (SR.SS) Method}

If the peak modal responses are assumed to be randomly phased they should exhibit weak correlation. In that instance, combination of the peak modal response by the simple sRss combination rule is appropriate. Recalling that in this study the rigid body component of the peak modal responses are being treated separately, the combination rule under discussion is used to combine only the damped periodic components of the peak modal responses.

The generic form of the equation for the combination of the damped periodic components is:

$$
R P=\left(\sum_{j=1}^{n} \sum_{k=1}^{n} C_{j k} R P_{j} R P_{k}\right)^{\frac{1}{2}}
$$

where the $c_{j k}$ are the modal coupling coefficients. For simple sRss combination

$$
\begin{aligned}
& c_{j k}=1.0 \text { for } j=k \\
& c_{j k}=0.0 \text { for } j * k
\end{aligned}
$$

and the combination can be expressed as: 


$$
R P=\left(\sum_{j=1}^{n}\left(R P_{1}\right)^{2}\right)^{k}
$$

In the NRC accepted Grouping and TAn weicent Methods, the combination rule is identical to the above with the following addition being made to the sum:

$$
\sum_{j=1}^{n} \sum_{k=1}^{n} R P_{j} R_{k}
$$

where the summation is crer all $\mathrm{j}$ and $\mathrm{k}$ modes whose frequencies are closely spaced. The definition of closeness is slightly different for the two methods but is essentially all modes within ten percent of each otiner.

In this study a base line response spectrum estimate is derived using the Reg. Guide 1.92 grouping method and used to gauge the results of the candidate methods. It is referred to as the Reg, Guide 1.92 solution.

\section{2 .2 Rosenblueth Double sum Combination (DSC) Method}

This method [10] is theoretically based in random vibration theory. As noted above, a variant of it, designated the double sum method, is one method deemed acceptable by the NRC staff for combining peak modal responses. For the DSC method the combination rule is:

$$
R P=\left(\sum_{j=1}^{n} \sum_{k=1}^{n} C_{j k} R P_{j} R_{k}\right)^{\frac{1}{2}}
$$

with the coupling coefficients defined as:

$$
C_{j k}=\left(1+\left(\left(\omega^{\prime},-\omega_{k}^{\prime}\right) /\left(\beta^{\prime}, \omega_{j}+\beta_{k}^{\prime} \omega_{k}\right)\right)^{2}\right)^{-1}
$$

where

$$
\begin{aligned}
& \omega_{j}^{\prime}=\omega_{j}\left(1-\beta_{j}^{2}\right)^{3 / 2} \\
& \beta^{\prime}{ }_{j}=\theta_{j}+2 / t \omega_{j}
\end{aligned}
$$

and $\omega_{1}$ and $\beta_{1}$ are the modal frequency and the damping ratio for the $j$ th mode respectively and $t$ is the duration of the strong motion segment of the seismic excitation. The coupling coefficient is based on representing the earthquake by a finite segment (duration 
$t)$ of the white noise. The value of duration must be specified for this method. In this study uniform damping was assumed (all $\beta_{1}$ are identical) and the duration was estimated from the Husid plot [13] for each seismic excitation record.

In the NRC accepted version of the procedure, the combination is given by:

$$
R P=\left(\sum_{j=1}^{n} \sum_{k=1}^{n} C_{j k}\left|R P_{j} \| R P_{k}\right| !^{3 / 2}\right.
$$

with the coupling coefficients as defined above. As can be seen the accepted version is identical to the Rosenblueth Method except for the use of the absolute summation within the radical. As such, the NRC Double sum method can be assumed to provide response estimates similar tco, but more conservative than the DSC method. The NRC Double sum method was not evaluated in this study.

\subsubsection{Complete Quadratic Combination (CQC) Method}

This method [11] is also theoretically based in random vibration theory. It differs from the DSC method in the definition of the coupling coefficients. That is:

$$
R P=\left(\sum_{j=1}^{n} \sum_{k=1}^{n} C_{j k} R P_{j} R P_{k}\right)^{\frac{1}{2}}
$$

and

$$
C_{j k}=\frac{8\left(\beta_{j} \beta_{k} \omega_{j} \omega_{k}\right)^{3 / 2}\left(\beta_{j} \omega_{j}+\beta_{k} \omega_{k}\right) \omega_{j} \omega_{k}}{\left(\omega_{j}^{2}-\omega_{k}^{2}\right)^{2}+4 \beta_{j} \beta_{k} \omega_{j}\left(\omega_{k}\left(\omega^{2}{ }_{j}+\omega^{2}{ }_{k}\right)+4\left(\beta^{2}{ }_{j}+\beta_{k}^{2}\right) \omega_{j}^{2} \omega_{k}^{2}\right.}
$$

where $\omega_{j}$ and $\beta_{j}$ are the natural frequency and damping ratio respectively for the $j$ th mode. The coupling coefficient is based on representing the earthquake by white noise of infinite duration. It can be shown that when the two modes have identical damping values, and $t=\infty$ is substituted in the Rosenblueth, equation the coefficients developed in the CQC or DSC methods are identical.

Although it is not obvious from the equations, the $C Q C$ method treats the effect of modal dainping more correctiy than the DSC method. It can be numerically shown that the effect of different damping for each mode on the coupling coefficient is significant, that the CQC equation accounts for it, and the Rosenblueth equation does not. A modified Rosenblueth equation that considers the effect of different damping values (and the finite duration) is given in reference 6 . 
The problem set consisted of the same six piping systems used in the BNL evaluations of the Independent support Motion (ISM) method. The systems are identified as; the RHR piping model, the AFW piping model, the z-bend piping system model, and the BM1, BM2 and BM3 piping system models. Each of these system models are fully described in the ISM reports, NUREG/CR-3811 [14] and NUREG/CR-5105 [2]. A brief description will be provided here.

The RHR and AFW piping systems are housed in the containment structure of the zion Nuclear Power Plant in Illinois. The containment structure consists of the containment shell and a separate concrete internal structure supporting a four-loop PWR nuclear steam supply system. Details pertaining to this system were made available to BNL by LINL who analyzed it as part of the Zion SSMRP studies performed by Lawrence Livermore Laboratory for NRC [15]. The Zion RHR and AFW nodels were also used as problems in tile LLNL studies of PVRC damping, peak broadening [16] and response margins $[17,18]$.

The RHR model, shown in Figure 2, consists of a 12 inch, Sch. 40 and Sch. 160, pipe line header running from a wall anchor at the internal structure of the containment building to an anchor in the AFT complex and an 8-inch, Sch. 40, branch line from the Refueling Water storage Tank (RWST) nozzle to the 12-inch pipe. The pipe material for the entire system is stainless steel. The line is rated at a design temperature of $400^{\circ} \mathrm{F}$ and a design pressure of 75 psi. One isolation valve exists at the upper end of the model, near the containment wall. The system exhibits eighteen natural frequencies in the range from $4 \mathrm{~Hz}$ to $34 \mathrm{~Hz}$ with closely spaced modes appearing above $12 \mathrm{~Hz}$.

The AFW model, shown in Figure 3, consists of a 16-inch, Sch. 120, main feedwater (MFW) line running from a steam generator nozzle to a containment penetration, and a 3-inch, Sch. 160, Auxiliary Feedwater (AFW) branch line running from the 16-inch MRW line to a containment penetration. The entire line, rated for a design temperature of $400^{\circ} \mathrm{F}$ and a design pressure of $200 \mathrm{psi}$, is carbon steel. The spring supports and snubbers are shown in the figure. This system exhibited 37 natural frequencies in the range from $3 \mathrm{~Hz}$ to $34 \mathrm{~Hz}$ indicating that it had significant lower frequency content.

As noted above, both the RHR and AFW piping systems were evaluated in the Zion SSMRF studies. In those studies statistical methods were used to develop a data base of seismic excitation sets suitable for these problems. In the ISM study the response of these problems were determined for thirty three different sets of seismic excitation from that data set. In this evaluation, thirtythree, ID (X direction), uniform support motion, excitation sets were extracted form the ISM input data set. Solutions 


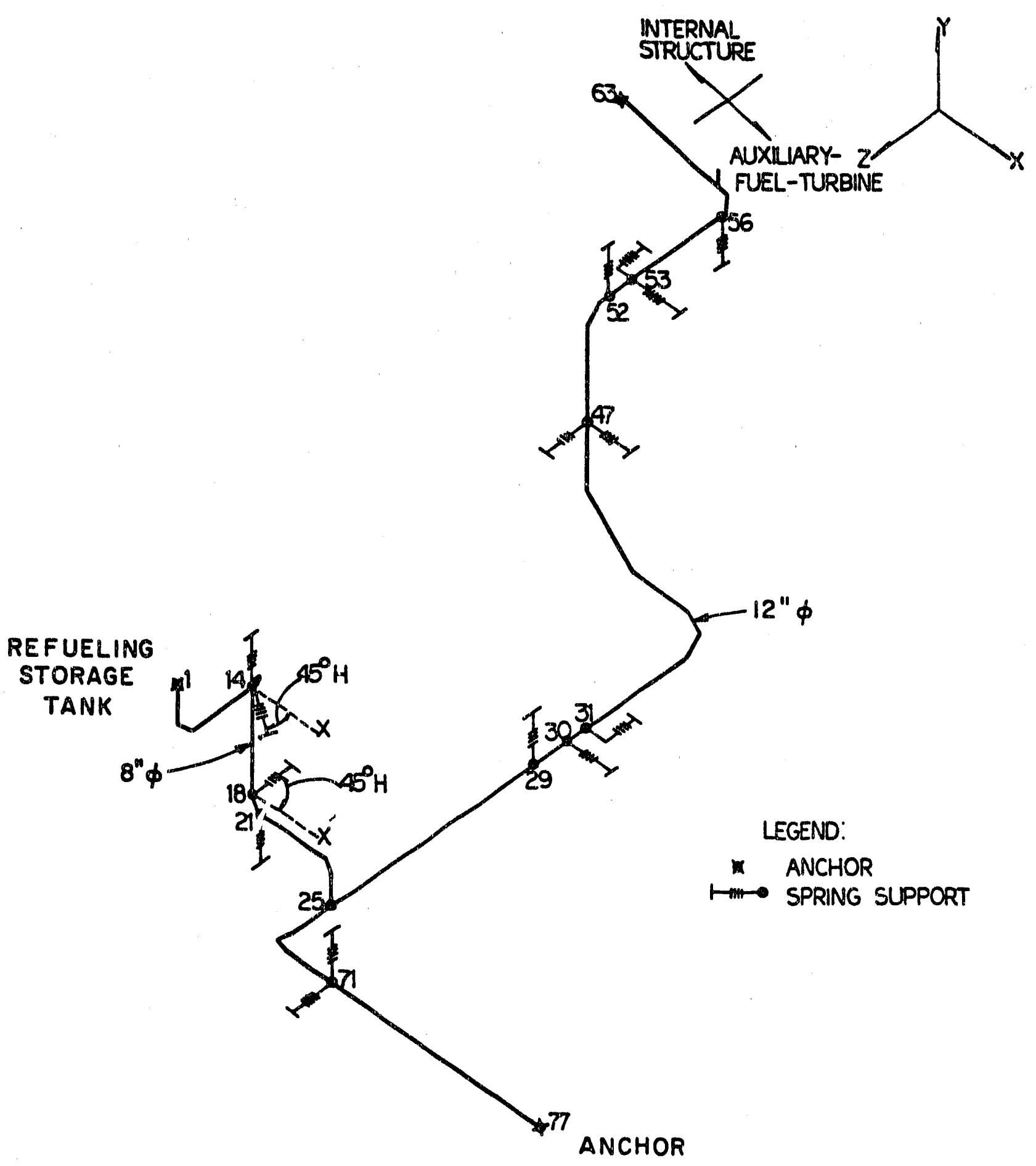

Figure 2 - RHR Piping Model 

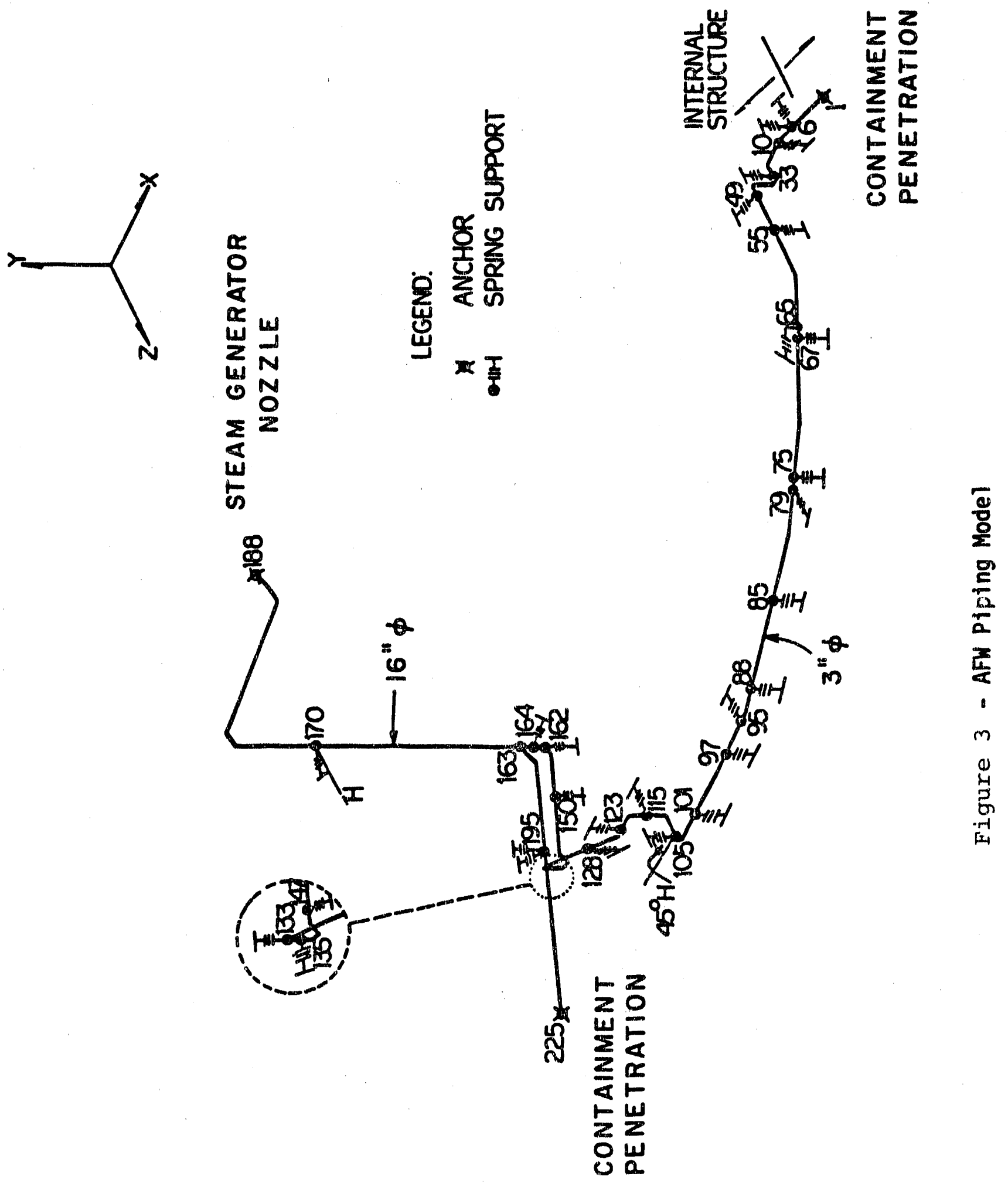
corresponding to each of these thirtythree inputs and 2 o uniform damping were developed for each problem providing some statistical basis to the study results.

The $\mathrm{Z}$ bend is a simple, planar configuration of 4-inch, Sch. 40 steel pipe consisting of two elbows and three straight lengths, as depicted in Figure 4. This system simulates a system used in laboratory tests to determine the seismic margins inherent in piping systems. It is a stiff system exhibiting 10 natural frequencies in the range from $9 \mathrm{~Hz}$ to $160 \mathrm{~Hz}$. For the tests the pipe was supported from and excited by three hydraulic actuators designed to drive the pipe uniformly. In this evaluation the test was simulated by imposing uniform excitation of the three supports in the $X$ coordinate direction and using $2 \%$ uniform damping.

The BMI and BM2 evaluations both involve the piping model shou'n in Figure 5. The piping consists of 2-inch and 6-inch, Sch. 40, carbon steel pipe, with a configuration corresponding to a line from a cooling water system in a nuclear power plant. It exhibited 27 natural frequencies ranging from $5 \mathrm{~Hz}$ to $9 \mathrm{~Hz}$. In BMl tile piping was considered to be part of a PWR system and the inputs to the model were derived from a 3D analysis of a PWR containment and internal structure. For BM2 the piping was assumed to be part of a BWR system. The inputs for this problem were derived from a $1 D$ stick model of a BWR structure. In this evaluation the response of the piping system was determined considering a uniform excitation of all supports in the $x$ coordinate direction that was first consistent with the inputs for the PWR model, BMI, and then corisistent with the inputs for the BMR model, BM2. In both cases 1\% uniform damping was used.

The BM3 model, Figure 6 , is a portion of a piping system from the HFBR Test Reactor at BNL. It consists of 3 inch, 4 inch and 8-inch, Sch. 40, stainless steel pipe. It exhibited 32 natural frequencies ranging from $3 \mathrm{~Hz}$ to $84 \mathrm{~Hz}$. The inputs for this model were derived from a two dimensional frame model with soil springs of the reactor building internal structures. For this evaluation, the response of the piping was determined for uniform excitation of all supports in the $X$ coordinate direction. The excitation was consistent with the inputs derived with the frame model and with if uniform damping.

A summary of model parameters are presented in Table 1. 


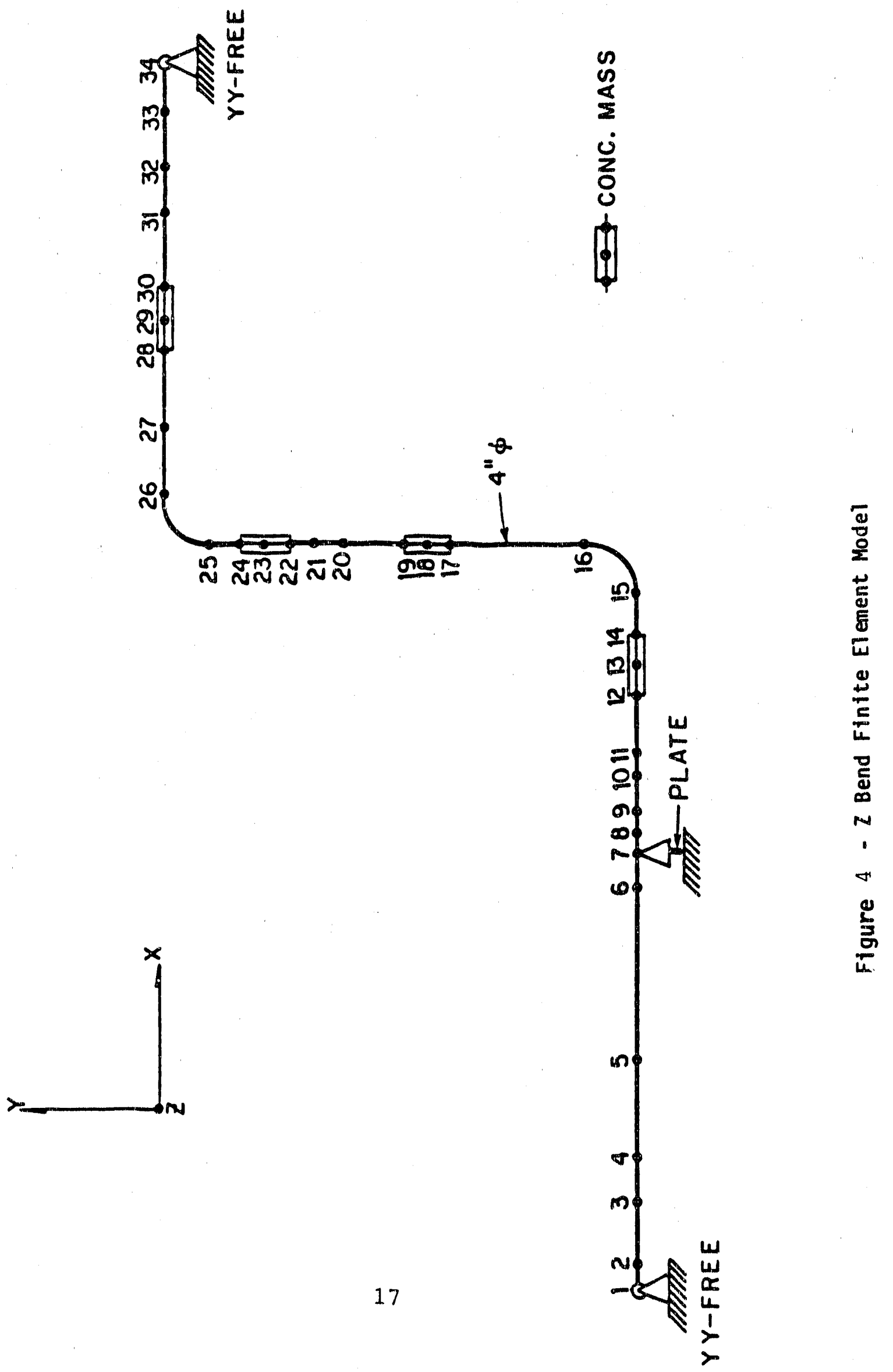



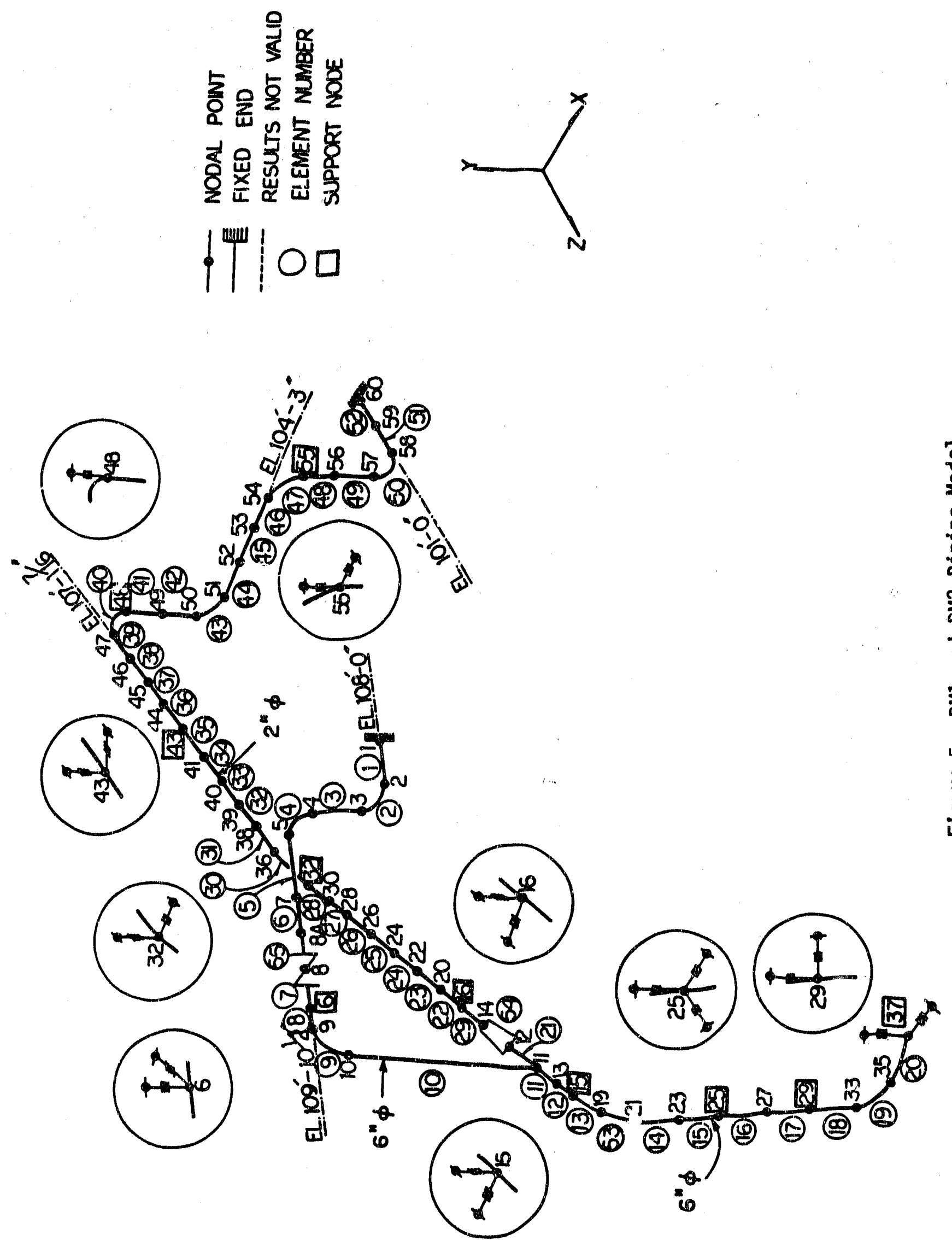


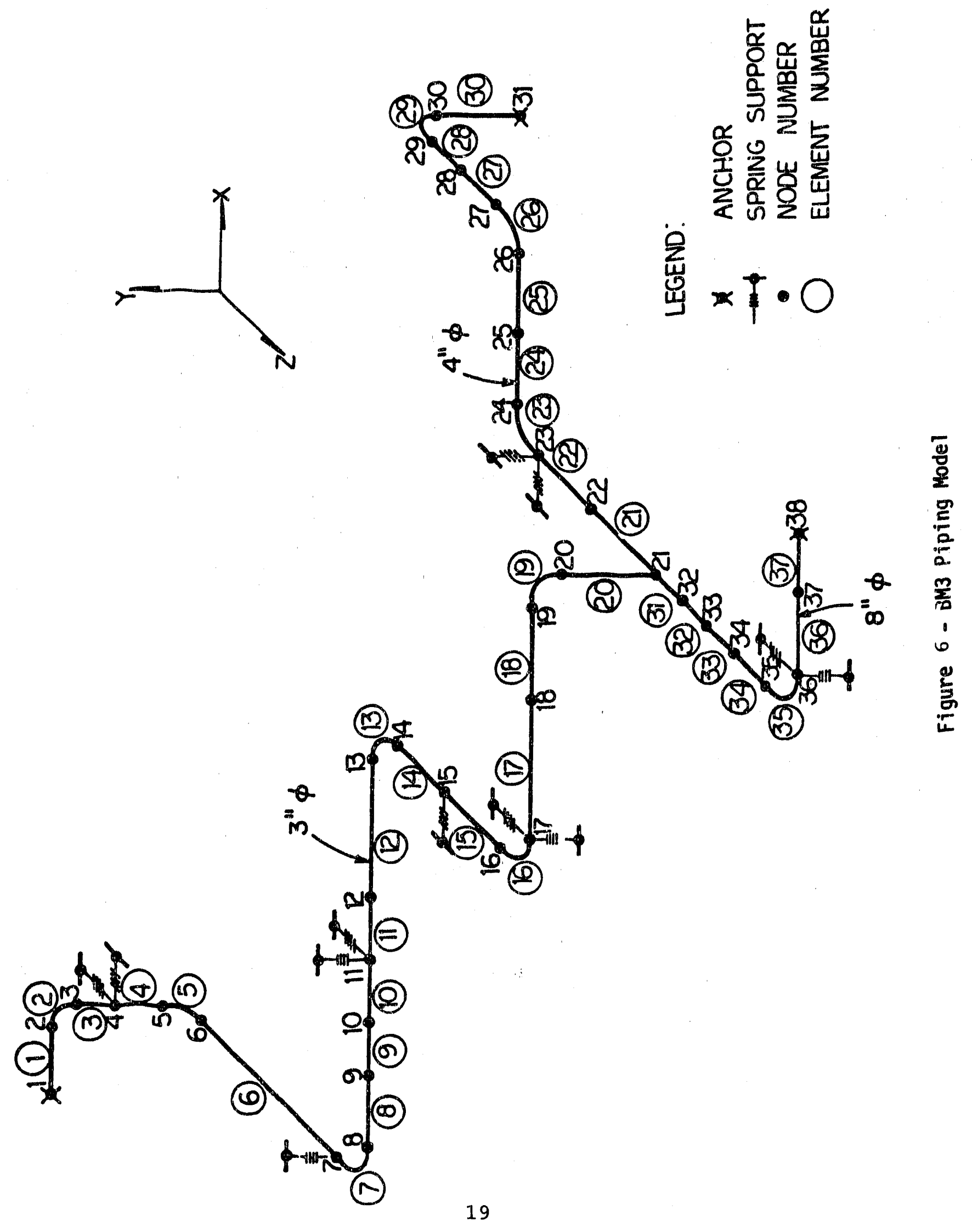




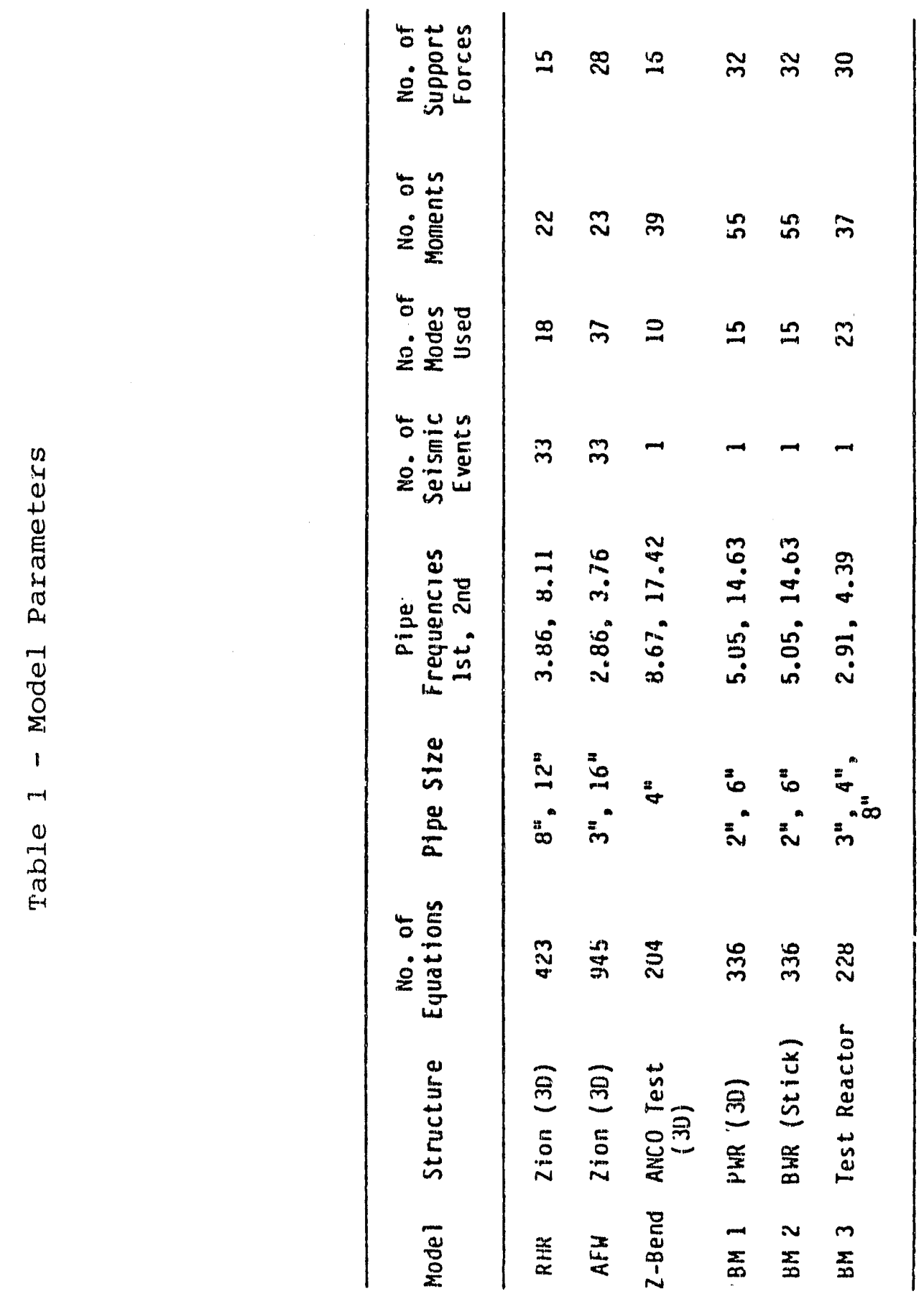


In this study, the adequacy and impact of alternate methods to combine between modal components are investigated. To assure that the impact of these methods and the differences between them is not obscured by other aspects of the calculational procedure, certain simplifications to the normal risponse spectrum calculational rnode were made. Specifically, in both the response spectrum and time history analyses, only uniform excitation of all supports in one coordinate direction, the $x$ direction, was considered. Further, in the response spectrum calculations, the input spectra was derived directly from the time history record used in the corresponding time history evaluation, with no broadening of that spectra being performed. Finally, the same level of uniform damping was used in both the time history and response spectrum evaluations.

By contrast, in a conventional response spectrum evaluation, broadened spectra would be defined for each coordinate direction, with each of these spectra being an envelope of the spectra for all supports in the system. In the conventional time history evaluation each support would be excited by its individual excitation. These excitations would be similar but not necessary identical.

As mentioned, uniform levels of damping were used in all response spectrum and time history evaluations. For the RHR, AFW and $Z$ bend problems, the level of damping used was $2 \%$ of critical. damping. For the BM1, BM2 and BM3 problems, the level of damping was $1 \%$ of critical damping.

All evaluations were performed at BNL. The time history evaluations for the RHR and AFW problems were developed using the SMACS computer code with all supports excited uniformly in the $x$ coordinate direction with a time history record that corresponded to the time history record used to excite the first support group in the earlier ISM studies [14]. The response spectrum evaluations for the RHR and $A F W$ problems and both the time history and response spectrum evaluations for the $Z$ bend, BM1, BM2 and BM3 problems were all performed using the computer code PSAFE2. Again for the $Z$ bend, BM1, BM2 and BM3 problems the excitation corresponded to that used to excite the first group for each problem in the ISM studies. In all response spectrum evaluations a cut off frequency of $100 \mathrm{~Hz}$ was used. For the time history evaluations, the modal superposition method of solution was used considering the same modes used in the response spectrum solutions. This was done to assure consistency between the time history and response spectrum solutions.

In the $\mathrm{Z}$ bend, $\mathrm{BM} 1, \mathrm{BM} 2$ and $\mathrm{BM} 3$ models, there are significant modes beyond the $100 \mathrm{~Hz}$ cut-off frequency used in the present study. For design purposes, the calculations for these models should have included the effect of missing mass - the residual 
rigid response (section 2.1.4). In this study it was not necessary because the same modes were considered in both the response spectrum and the time history methods. However if a correction for missing mass had been made in both the time history and response spectrum results developed in the study, the agreement between these solutions would have improved.

The inputs used in the evaluations are depicted in Figures 7 through 10. Figure 7 shows the support point acceleration time history record and the corresponding response spectra for the RHR problem. Two sets of records are shown on this figure corresponding to the response spectra exhibiting the minimum and maximum acceleration pears. The minimum peak acceleration is $0.74 \mathrm{G}$ occurring for record 19 while the maximum peak acceleration is 2.81G occurring for record 25. Similar data for the AFW problem are shown in Figure 8 . For that problem the minimum of $0.82 \mathrm{G}$ occurs for record 10 and the maximum of $2.81 \mathrm{G}$ again occurs in record 25. The support point acceleration time history record and corresponding response spectra for the $z$ bend are shown on the left side of Figure 9, while those for the BMI problem are shown on the right side of Figure 9. The input records for the BM2 and BM3 problems are shown on the left and right side, respectively, of Figure 10.

As will be noted when the results are reviewed, response spectrum estimates of response were dev'eloped based on the Gupta, Hadjian and Lindley-Yow methods to address frequency effects and the DSC, CQC and SRSS methods to combine over modes. In addition, response spectrum estimates of response based on the use of the Regulatory Guide 1.92 grouping method, were also developed. These were developed to permit a comparison of the new procedures to a currently accepted procedure. In reviewing the results based on Regulatory Guide 1.92 , it must be remembered that the results were developed using unbroadened spectra.

Again as will be noted when the results are reviewed, the specific candidate procedures are not evaluated separately. That is, there are no evaluations for the cupta meth d only or the DSC method only. Instead evaluations are made for each of the methods to address frequency correlation effects when coupled with each of the methods for treating closely spaced modes. Specifically, three response spectrum evaluations involving the Gupta method were performed for each time history record. Gupta coupled with DSC, Gupta coupled with $\mathrm{CQC}$ and Gupta coupled with SRSS. For each time history evaluation, then, there were nine response spectrum evaluations involving the candidate methods and an additional response spectrum evaluation involving the Regulatory Guide procedure. The result tables depict ten columns of response spectrum results corresponding to these evaluations. 

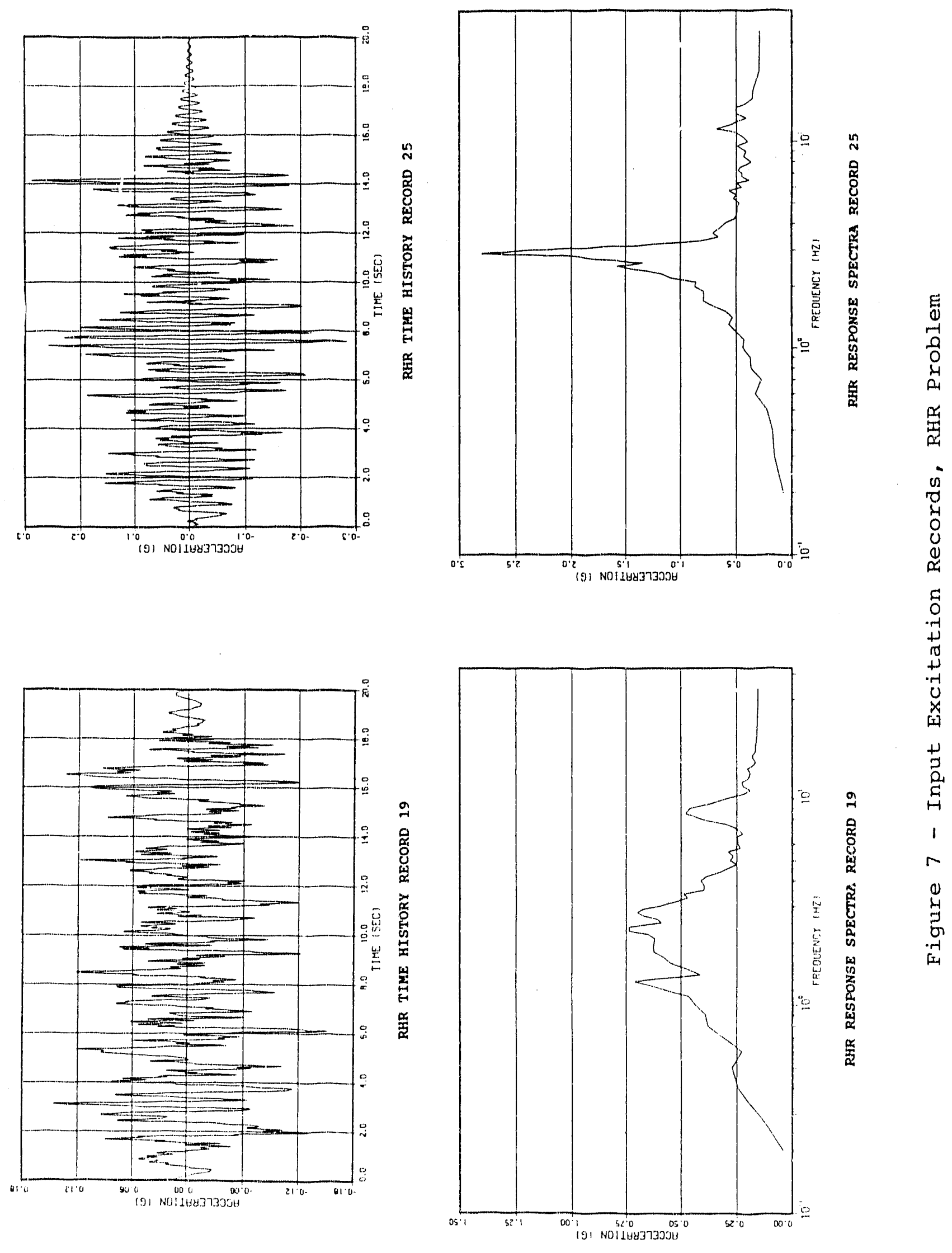

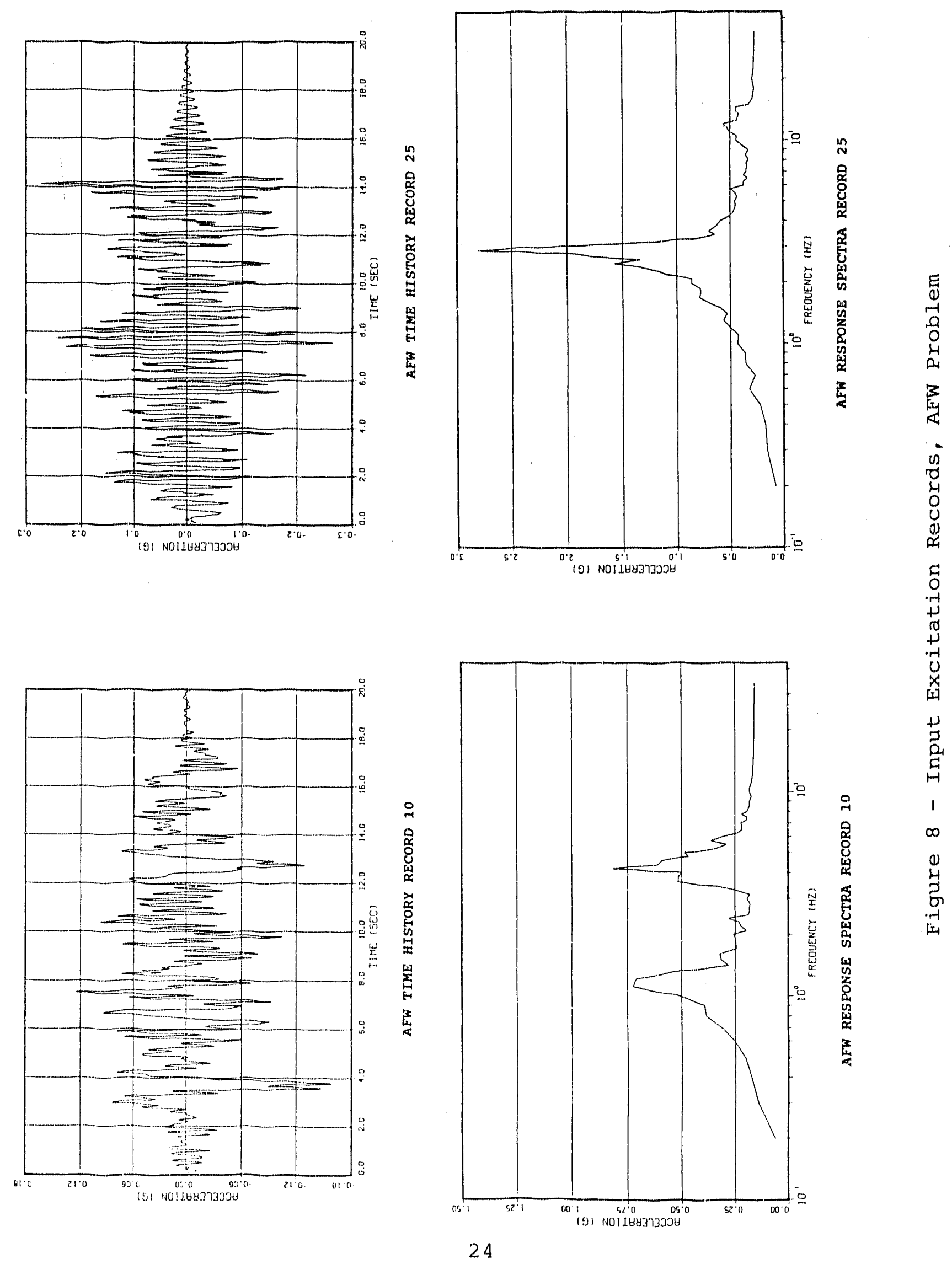

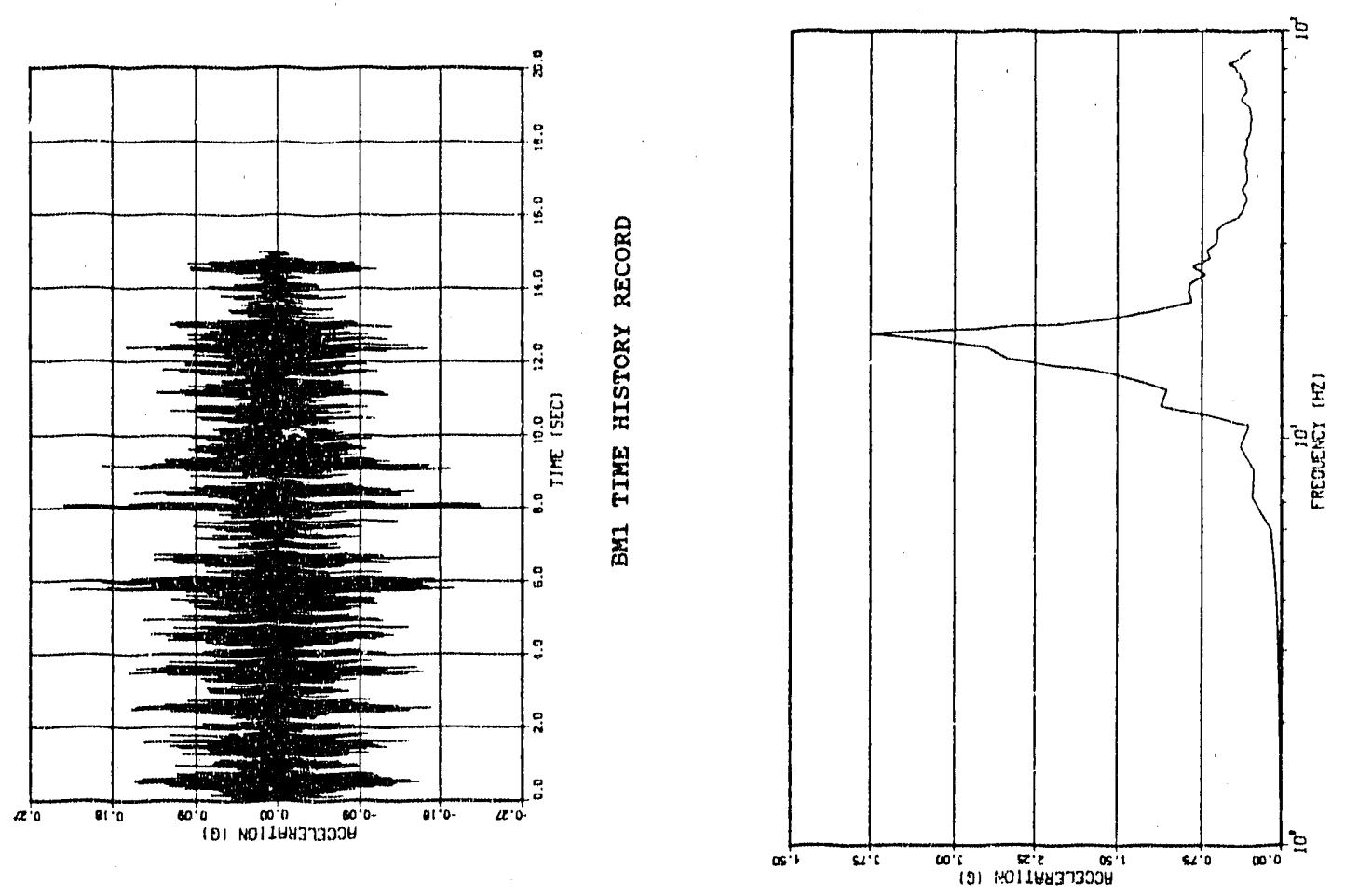

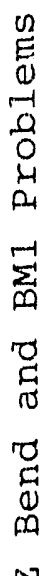
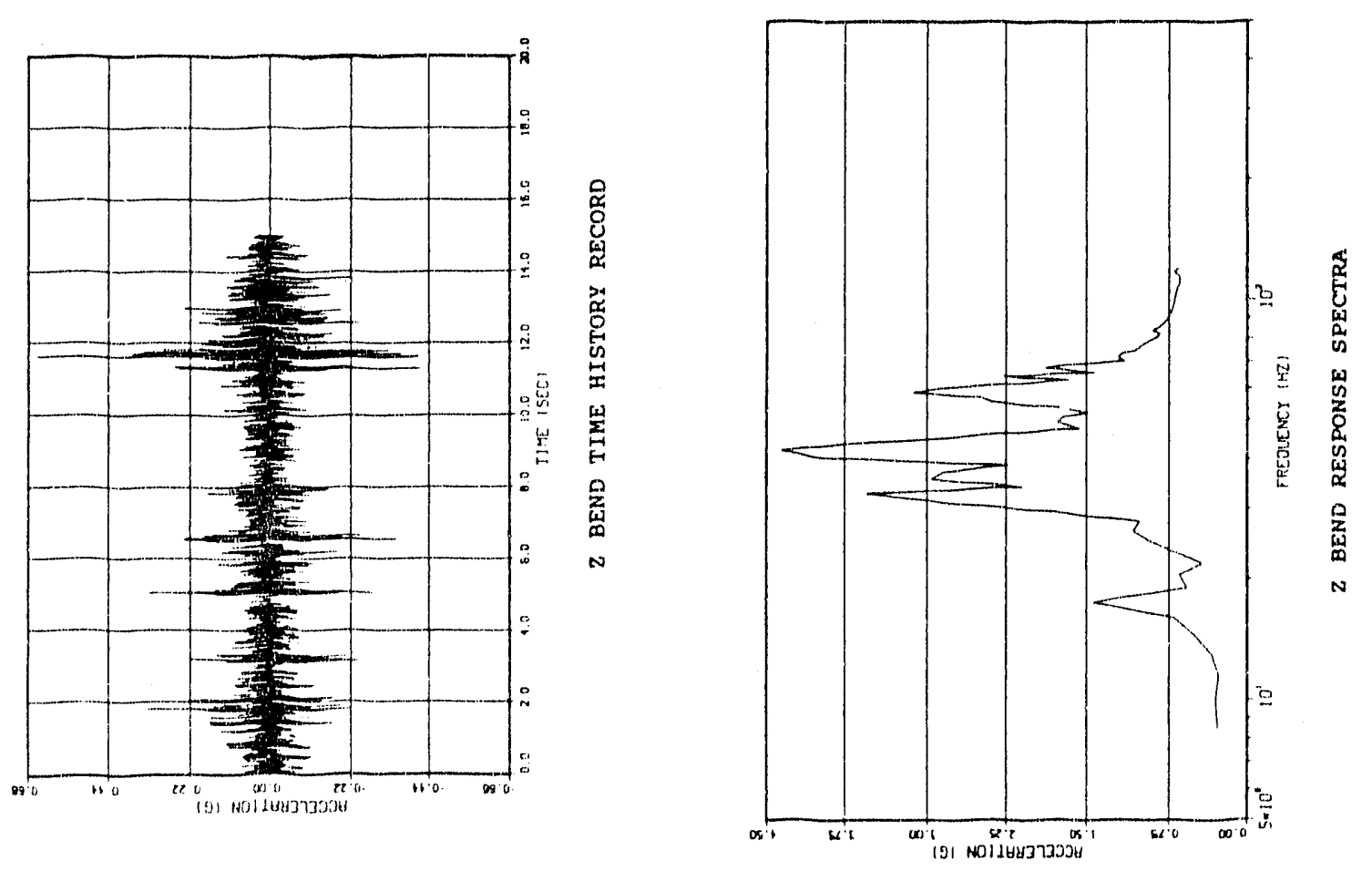

告

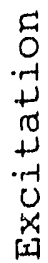

号

总 

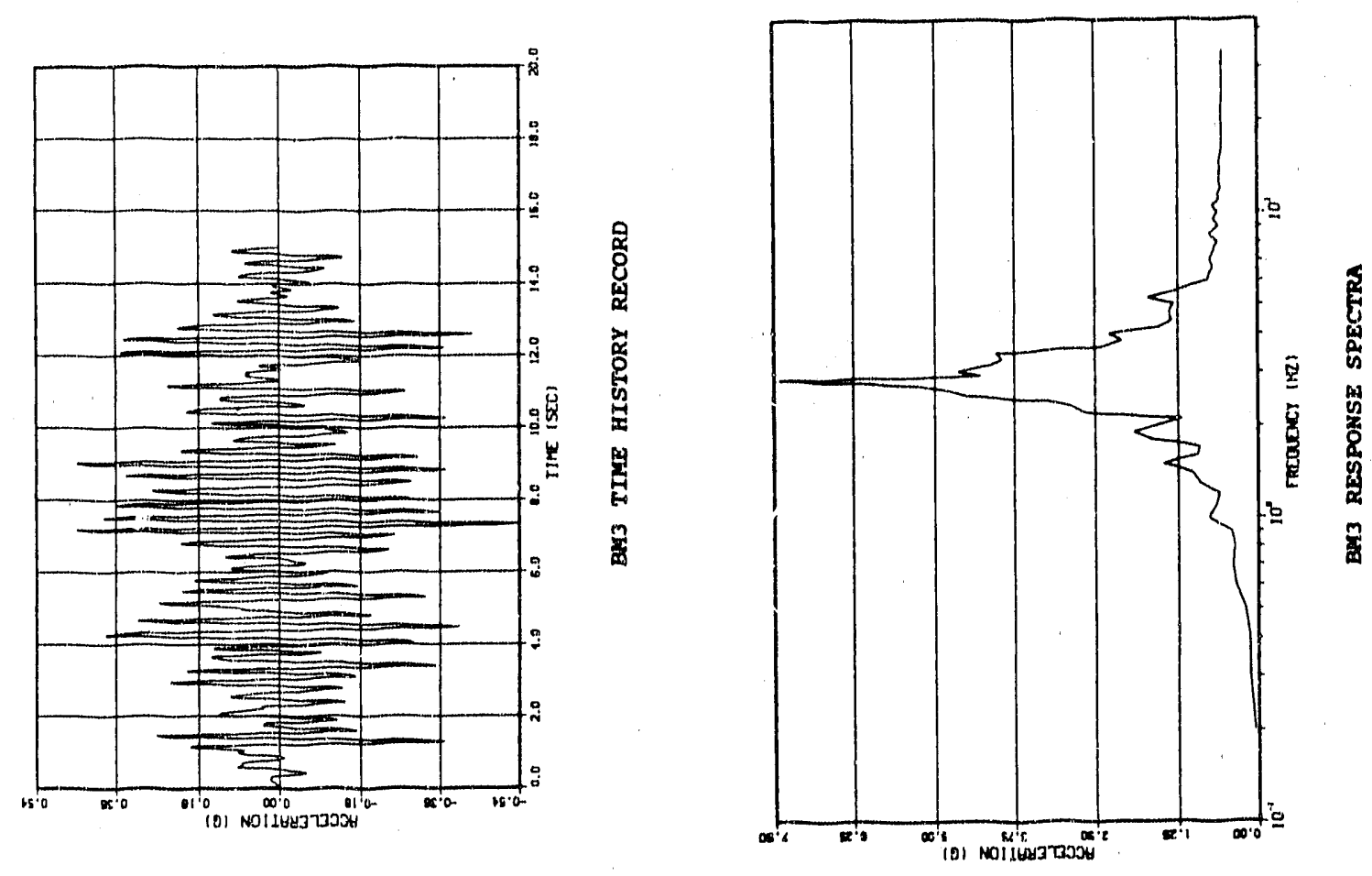

足
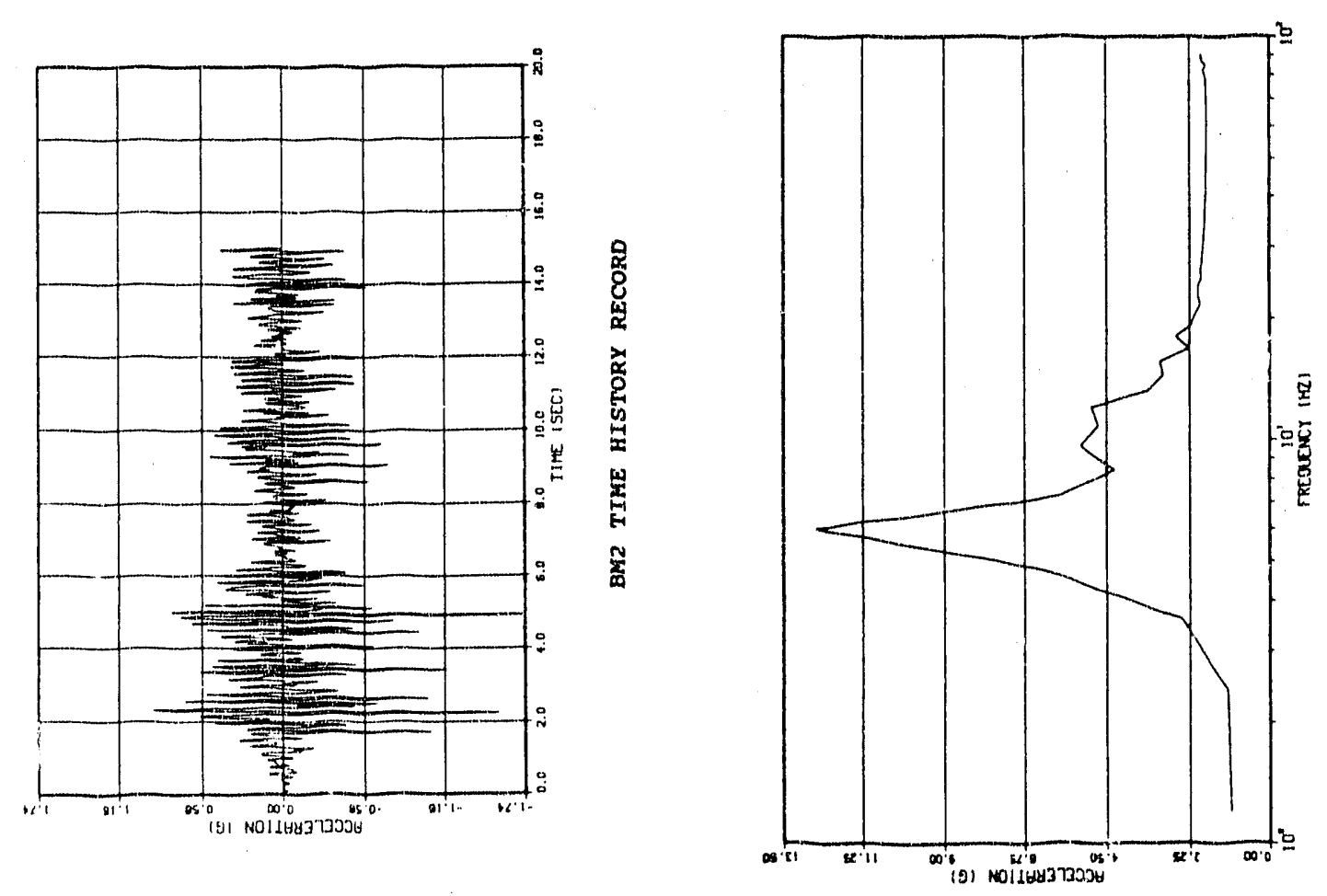

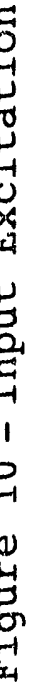




\section{STUDY RESULTS}

The basic results of the study are presented as tables in Appendix I. The tables list the maximum time history results as well as the corresponding response spectrum results for each candidate method and the Regulatory Guide Grouping procedure. separate tables are included for support force results and for pipe end moment results. As an example of these tables, the support force results for the RHR model and earthquake No. 3 are presented in Table 2 .

The first column indicates the element number for which the data applies while the second column lists a force code identifier (1 corresponds to a support force, 6,12 or 18 corresponds to a pipe end moment). The maximum value from the time history record of the response (force or moment) due to the particular earthquake is listed in the third column of the tables. Subsequent columns summarize the response spectrum results listed according to the particular methodology used to address frequency correlation effects, i.e., Gupta, Hadjian and Lindley-Yow. Three columns of values are shown under each of these methods corresponding to the method used to address closely spaced modes, i.e., DSC, CQC and SRSS. A last column shows the results that were obtained using the SRSS with clustering in accordance with Regulatory Guide 1.92. At the end of each column of response spectrum results, two more entries, corresponding to the mean value of the data in the column and the standard deviation of that data, are provided.

The response spectrum results presented in the tables are not actually the response quantities but rather the percentage by which the respective response spectrum (RS) estimate of a response quantity exceeded the time history (TH) estimate of that quantity. This percentage, termed the Degree of Exceedance is defined as:

$$
\text { Degree of Exceedance }=100(\mathrm{RS}-\mathrm{TH}) / \mathrm{TH}
$$

If this value is equal to zero then the response spectrum and time history estimates are the same. If the value is negative, the response spectrum estimate is less than the time history estimate.

The data in these tables were obtained at selected nodes and elements for each piping system. For the $\mathrm{Z}$ bend, BM1, BM2 and BM3 problems this included all nodes and elements. For the RHR and AFW problems, only a selection corresponding to points where peak stresses would be expected to occur, such as at elbows reducers and tees, were included. Since the data in these tables should be peak values, comparisons due to various excitations and to the results of different combination methods should be significant. 


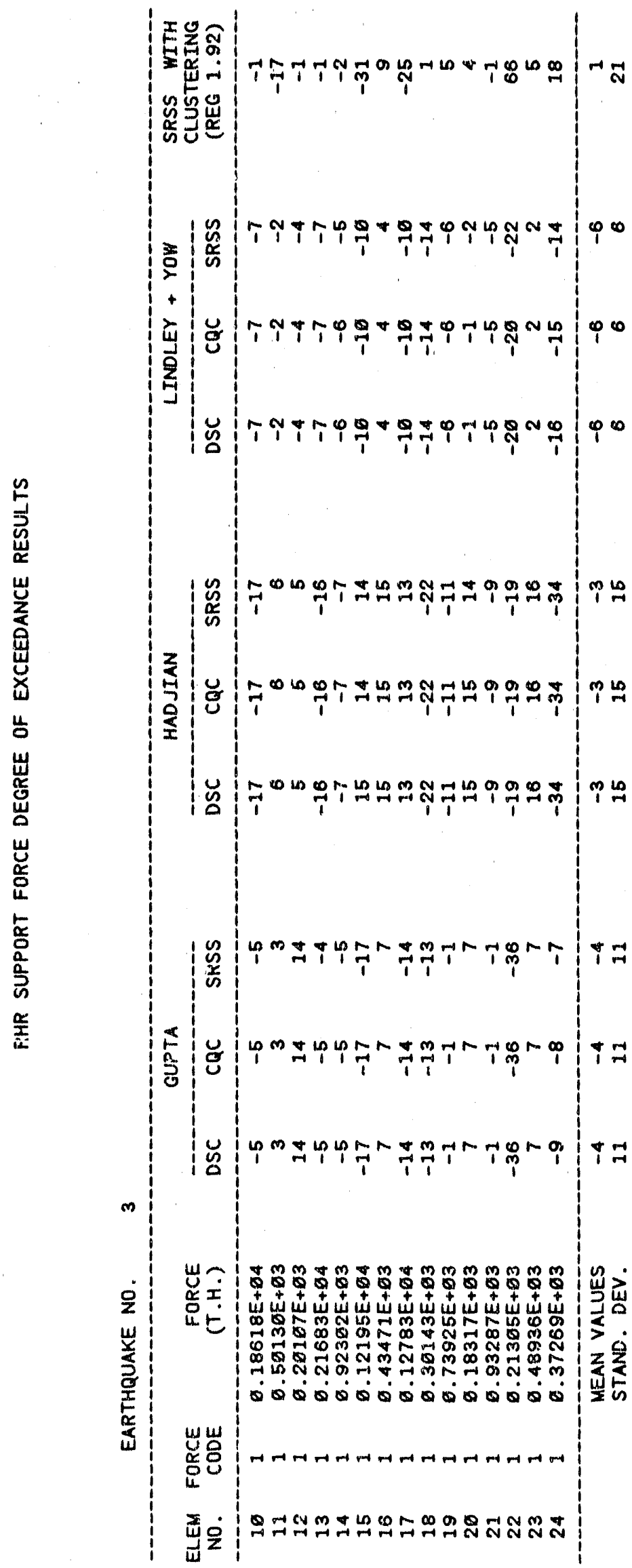




\subsection{RHR and AFW - Degree of Exceedance Results}

For each of these problems, sixty-six tables, corresponding to the thirty-three seismic events considered ior each problem, are included in Appendix I. These results were processed to obtain statistical properties. Table $3 a$ shows the mean value of the support force Degree of Exceedance results for the RHR problem. Table $3 \mathrm{~b}$ lists the corresponding standard deviations of the support force Degree of Exceedance results while Tables $3 \mathrm{c}$ and $3 \mathrm{~d}$ show the mean and standard deviation of the end moment Degree of Exceedance results for this problem. Tables 4 a through $4 \mathrm{~d}$ show the same type of information for the mean and standard deviation results for the AFW problem. The format of these tables is identical to that for the single seismic events, Table 2, with the column listing the time history values being deleted and the entries being the statistical estimates.

The mean value results for the RHR and AFW problems are also shown graphically in Figures 11 and 12 respectively. on each of these figures the support force results are shown on the upper plot while the end moment results are shown on the lower plot. Each point on each plot corresponds to a tabulated data point. All the data corresponding to a specific candidate method is shown in a column under a heading designating the method used to address frequency correlation effects and above a subheading designating the method used to treat closely spaced modes. The last column, with the subheading REG 1.92, depicts the estimates of results developed using the Regulatory Guide Grouping procedure.

These plots show the total range and dispersion of the data as a function of location since, for a candidate method, every plotted point corresponds to a different node or element. The distribution of results, and in particular the occurrence of equivalent results, is clearly depicted in the figures, with points corresponding to equivalent results being plotted adjacent to each other on a horizontal line. As an example, referring to the upper plot of Figure 12, six of the mean values of the support force degree of exceedance parameter, for the AFW problem, were essentially null for the Gupta method coupled with either the DSC, CQC or SRSS methods.

\subsection{BNL Problems - Degree of Exceedance Results}

Two tables are included in Appendix I for the $\mathrm{Z}$ bend, BM1, BM2 and the BM3 problems. One table presents the support force Degree of Exceedance results while the other table presents the end moment Degree of Exceedance results for each problem. The results are also shown graphically in Figures 13 through 16. These figures have the same format as Figures 11 and 12 with the difference being that in these figures the actual data for a single seismic event are shown. Results with magnitudes approaching zero are however not depicted in these figures. The elimination of these small 
responses was based on the considerations that first, they do not control design, and second, that they could introduce misleadingly large Degree of Exceedance values into the data plots. Inordinately large Degree of Exceedance values could occur since as the time history estimate of a response approached zero, division by zero could produce large values even for small differences between the response spectrum and time history estimates. It was felt that the inclusion of these large magnitude Degree of Exceedance estimates would cause a misleading increase in the dispersion of the data shown on the figures. 


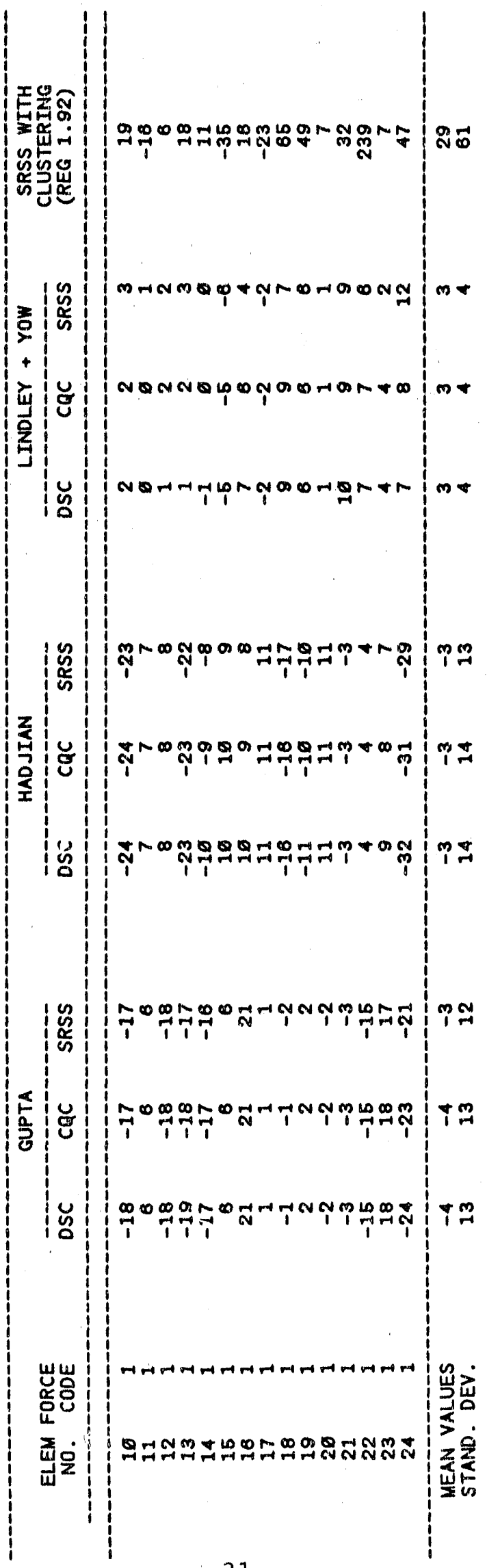




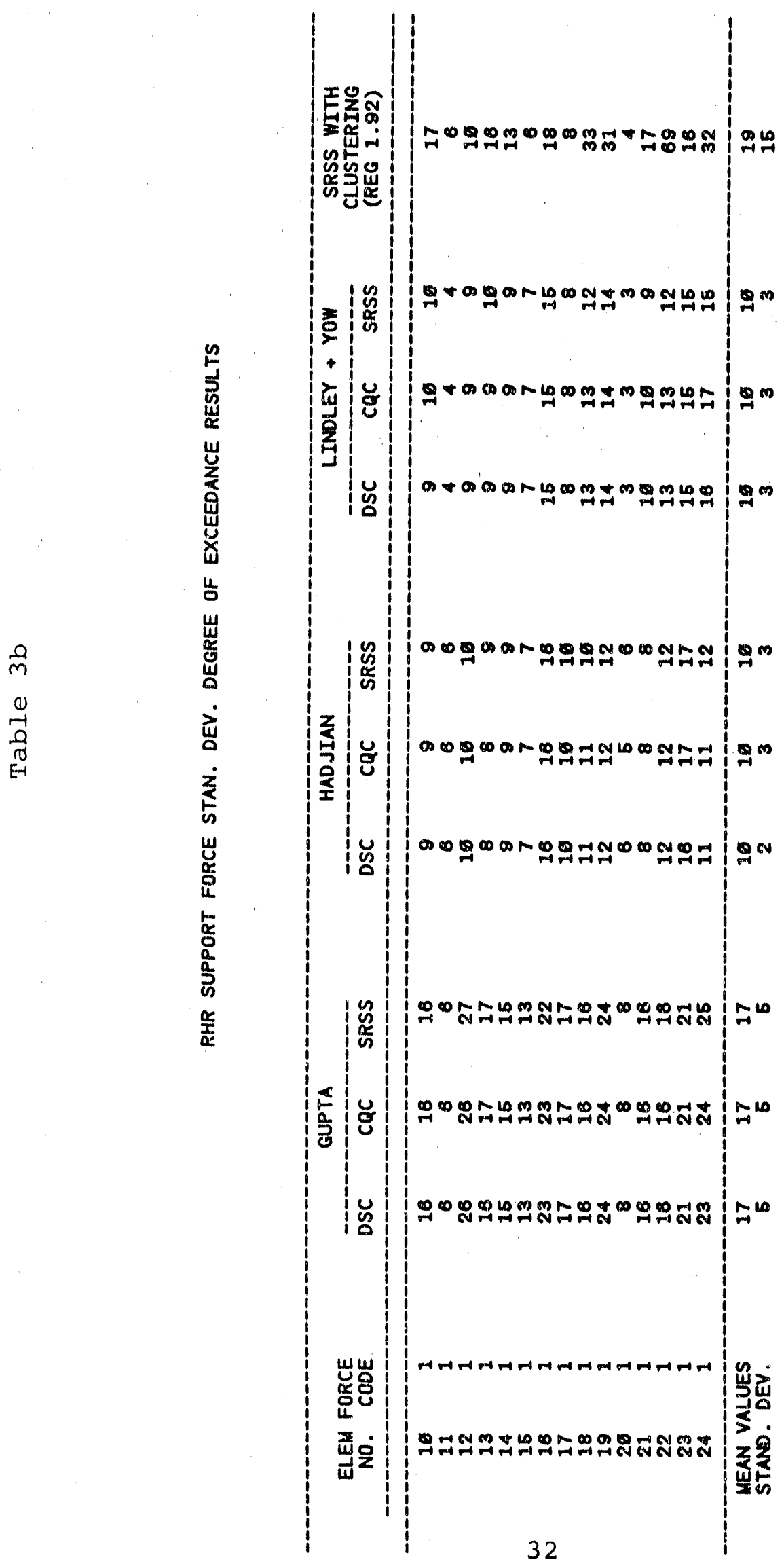




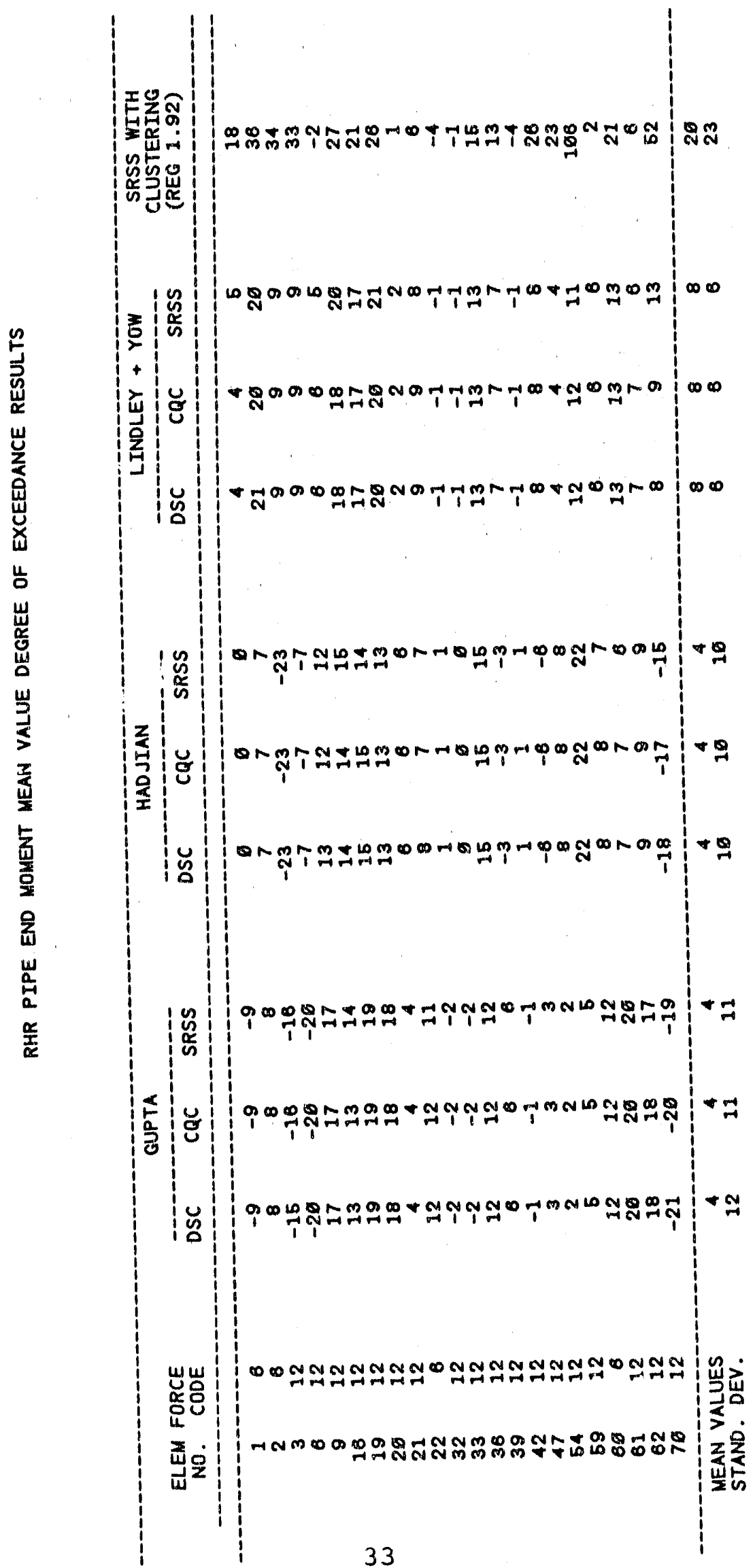




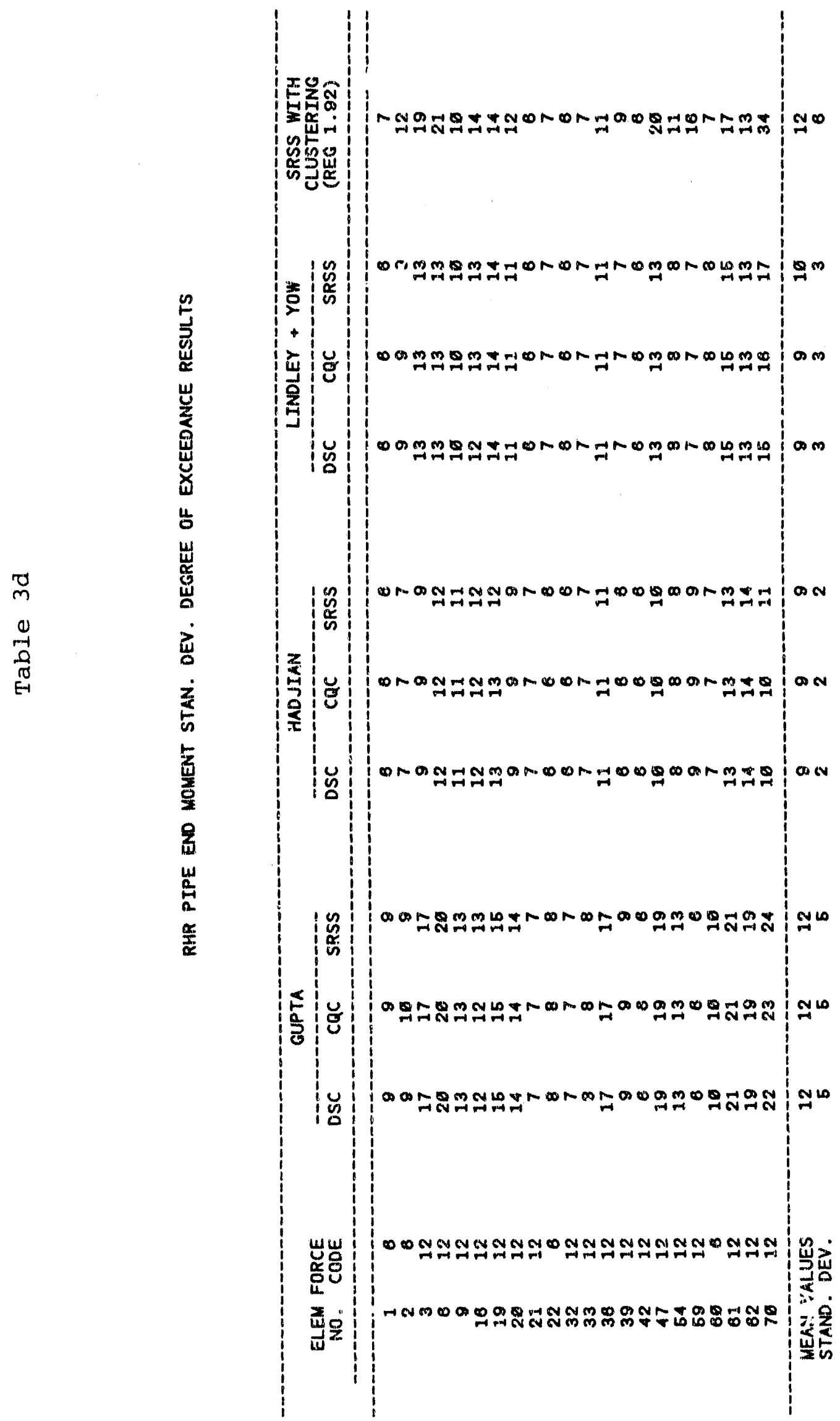




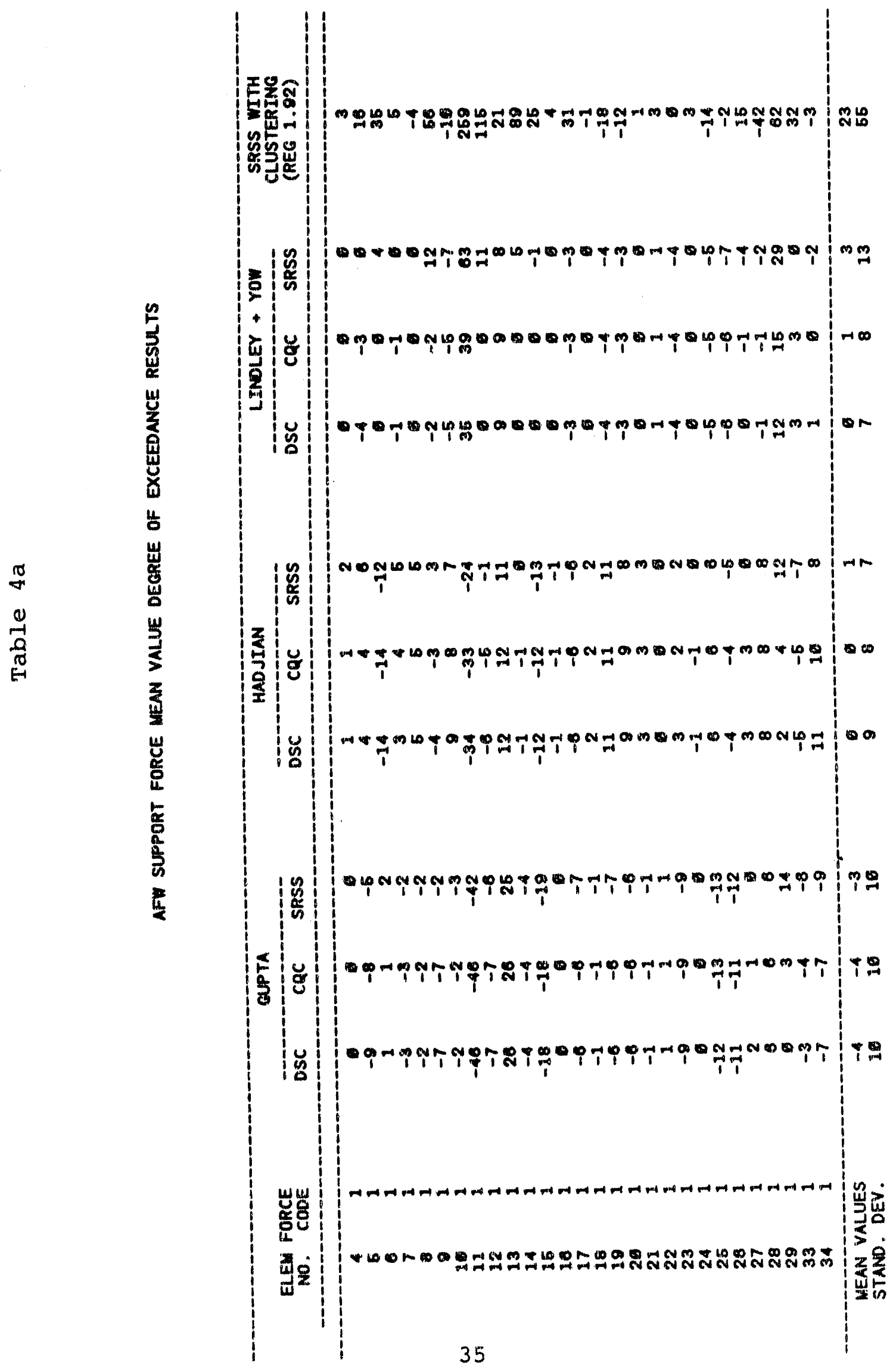




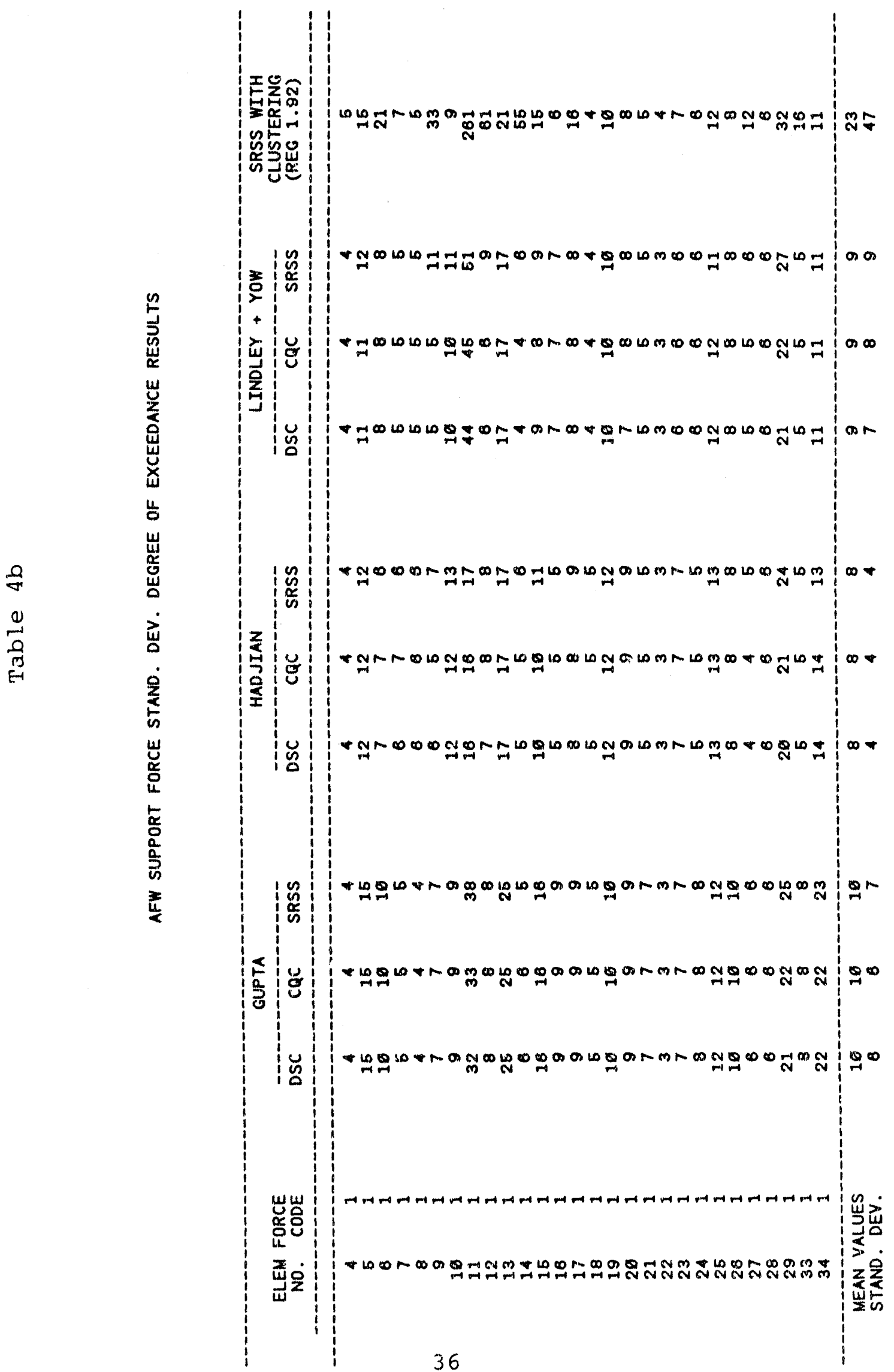




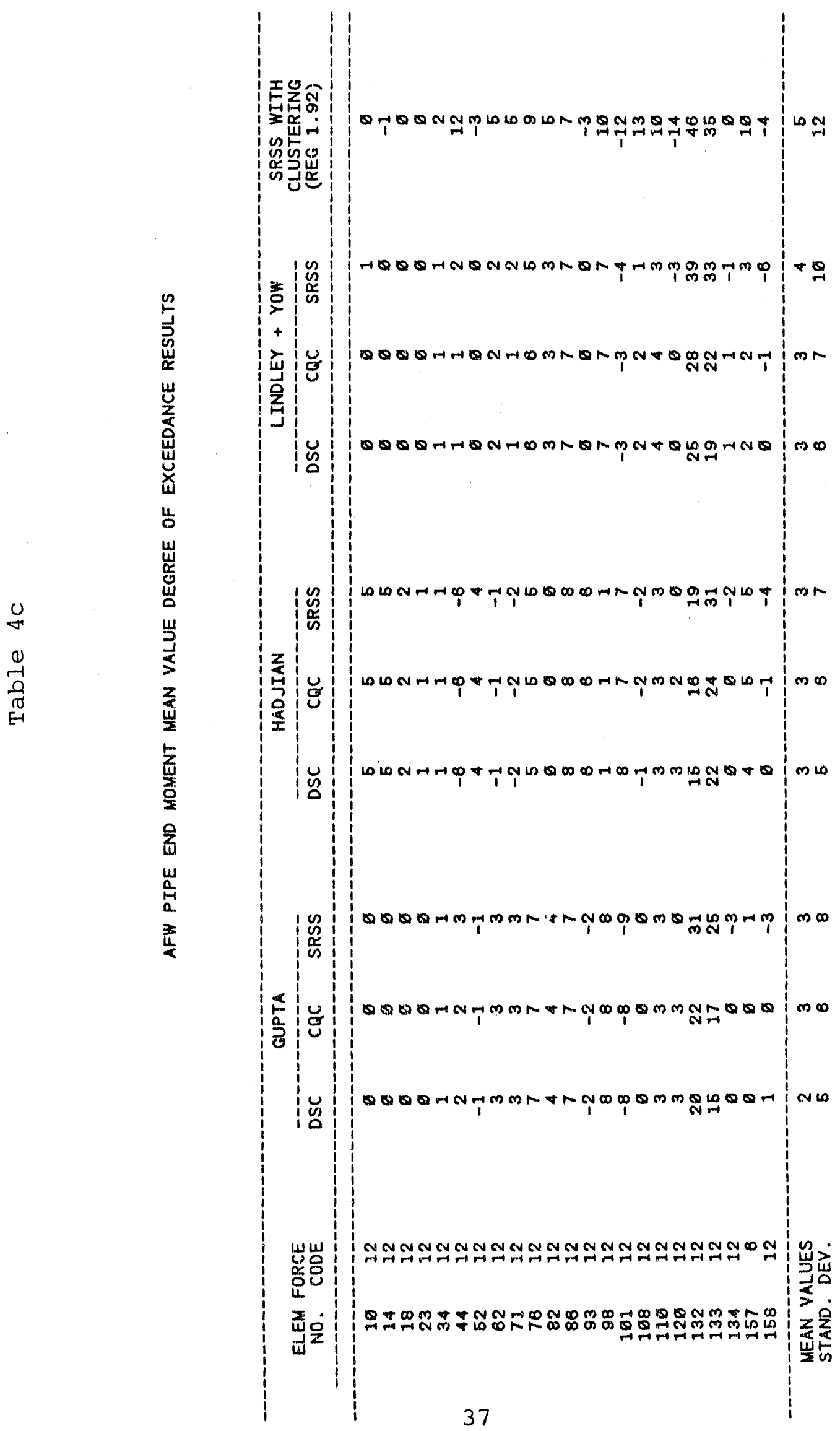




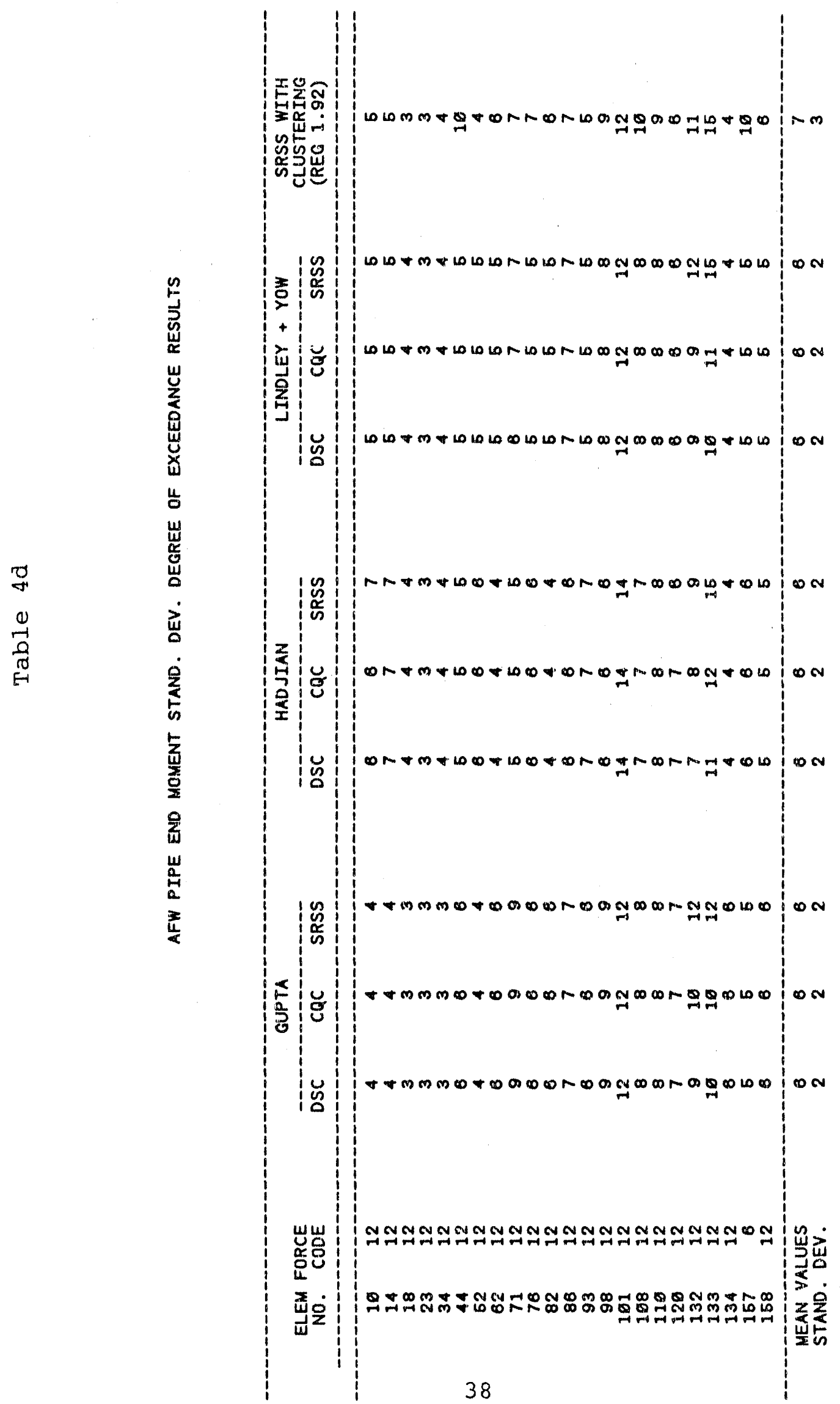




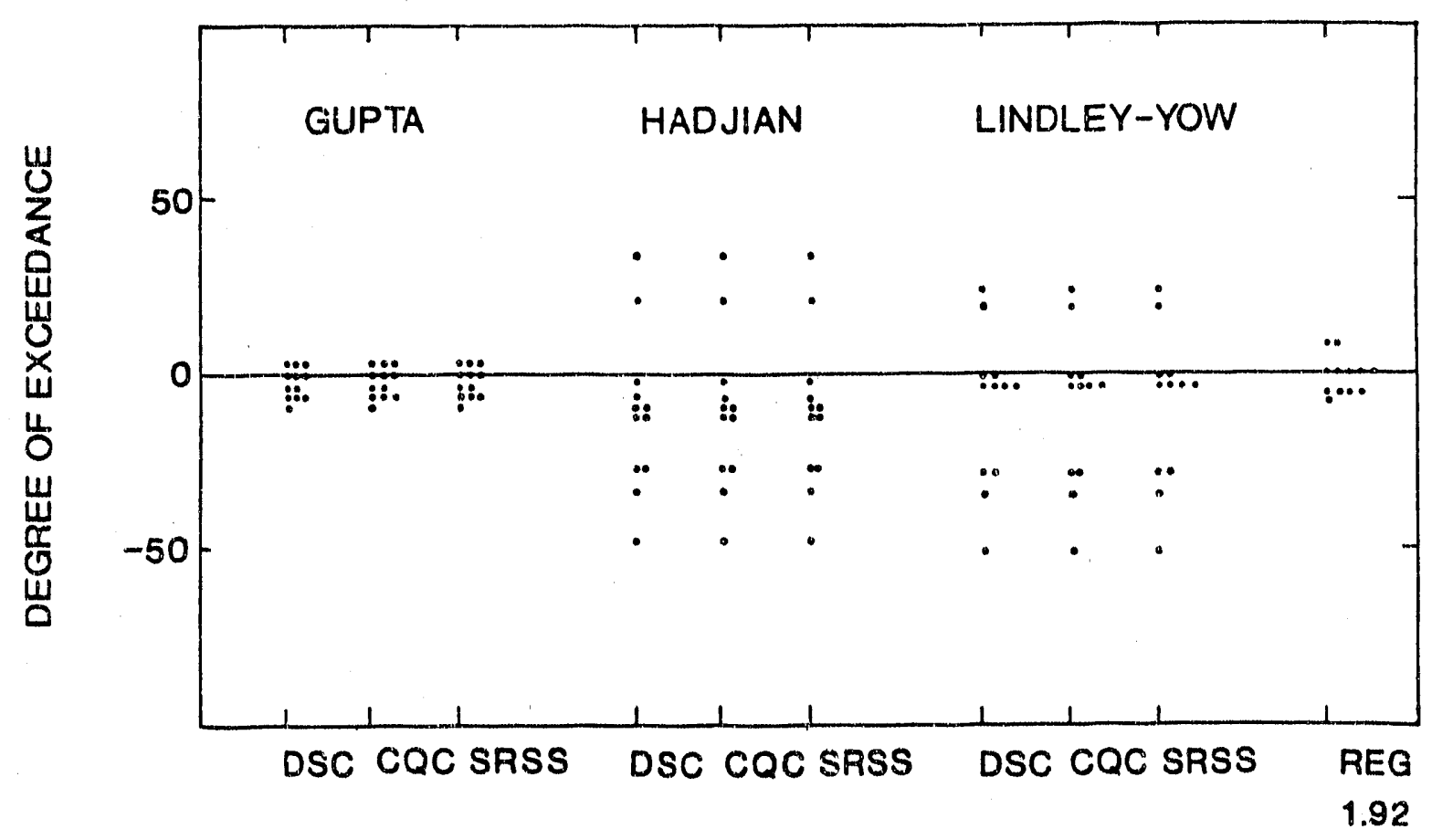

Z BEND SUPPORT FORCE RESPONSE

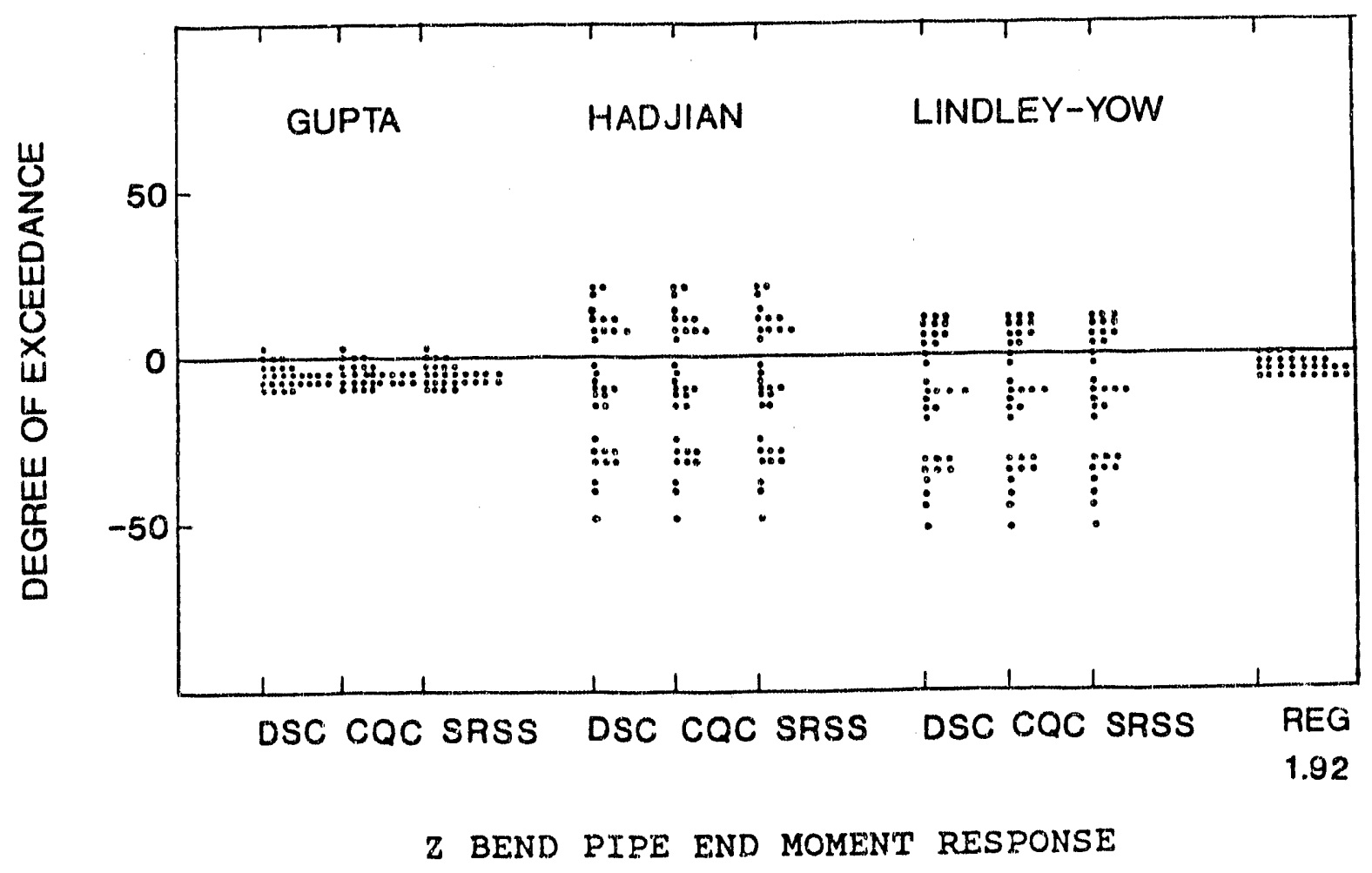

Figure $13-z$ Bend Problem Distribution of Results 

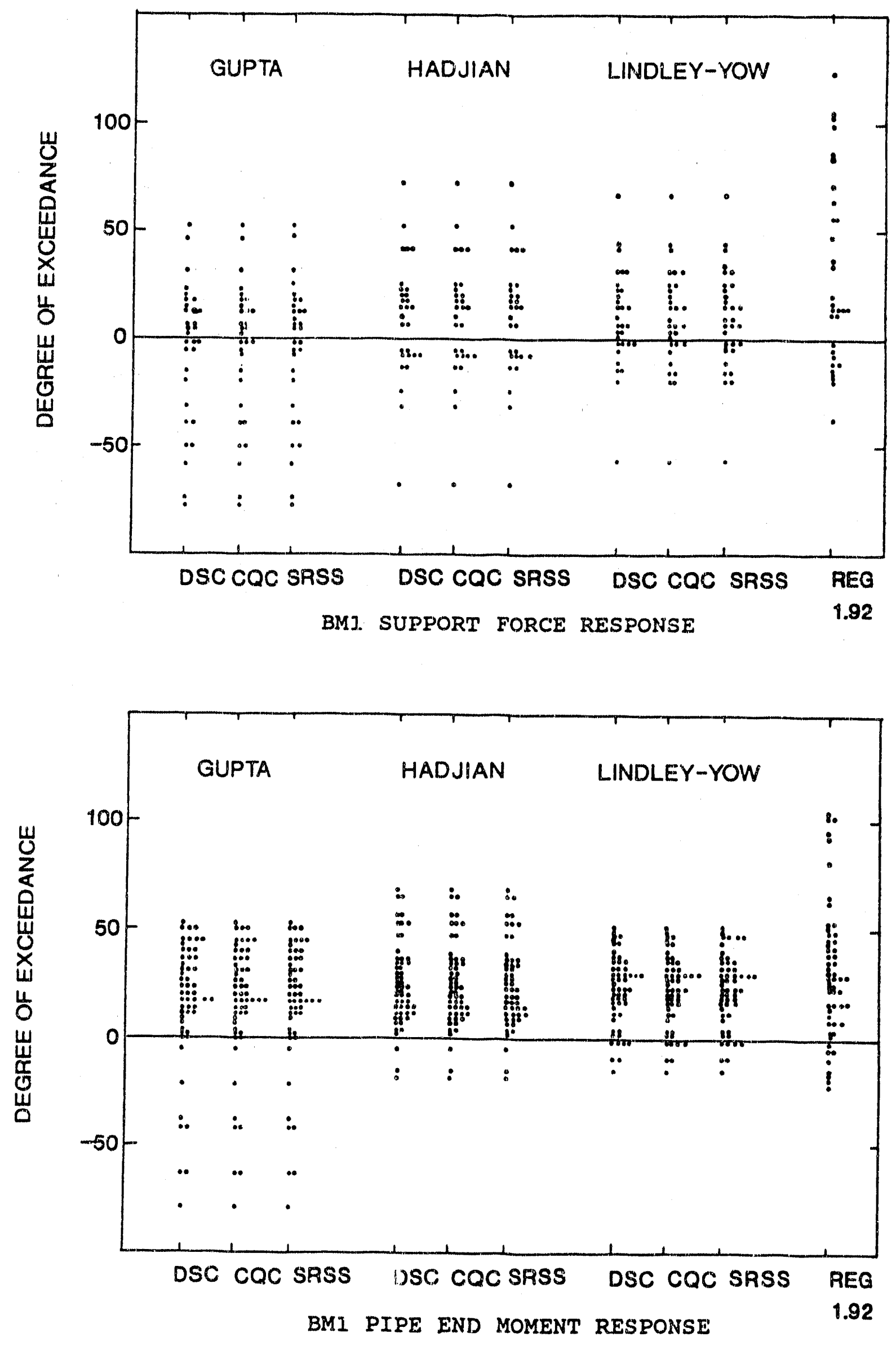

Figure 14 - BMi frólem Distribution ox Resuits 

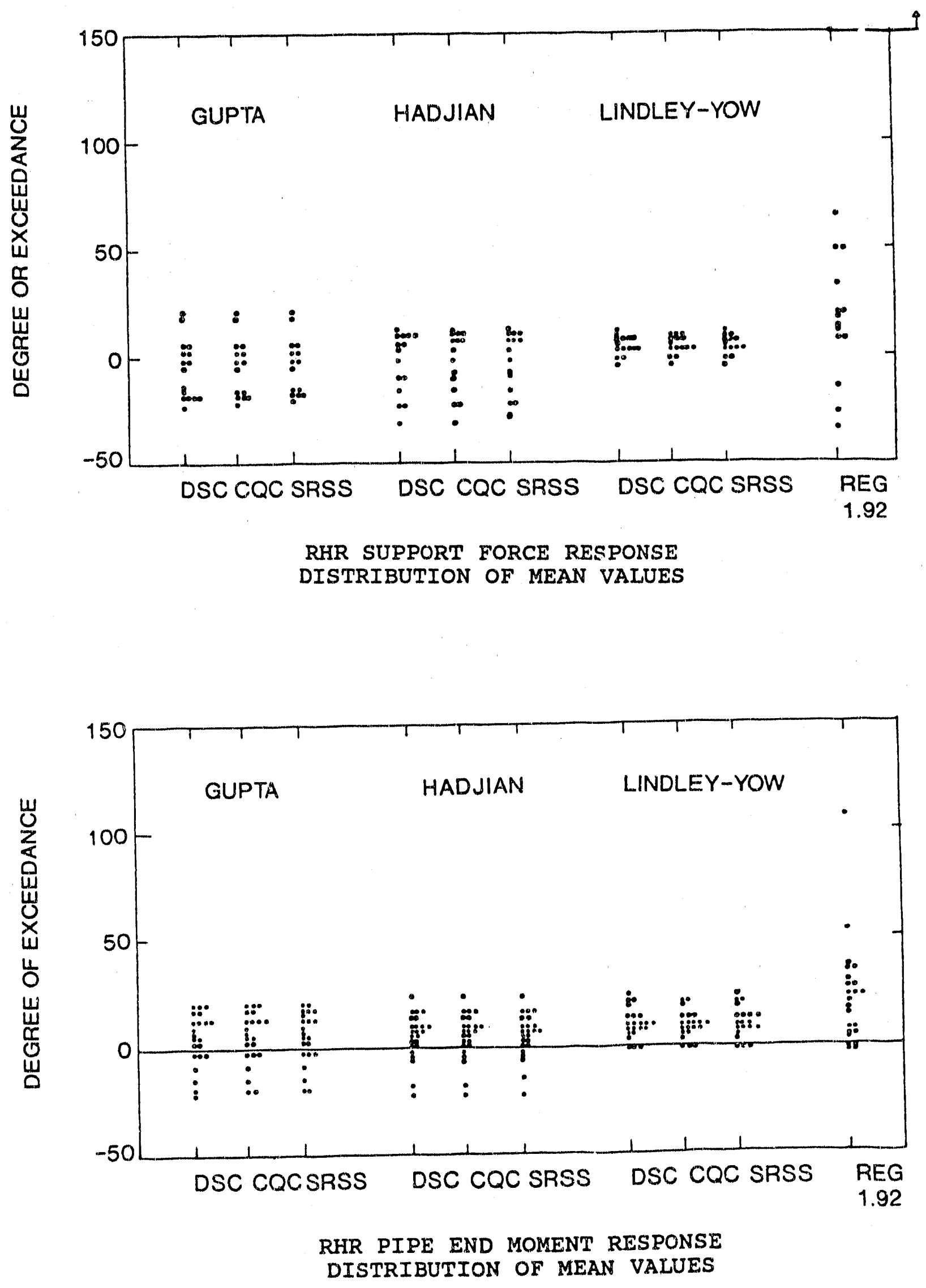

Figure 11 - RHR Problem Statistical Results 

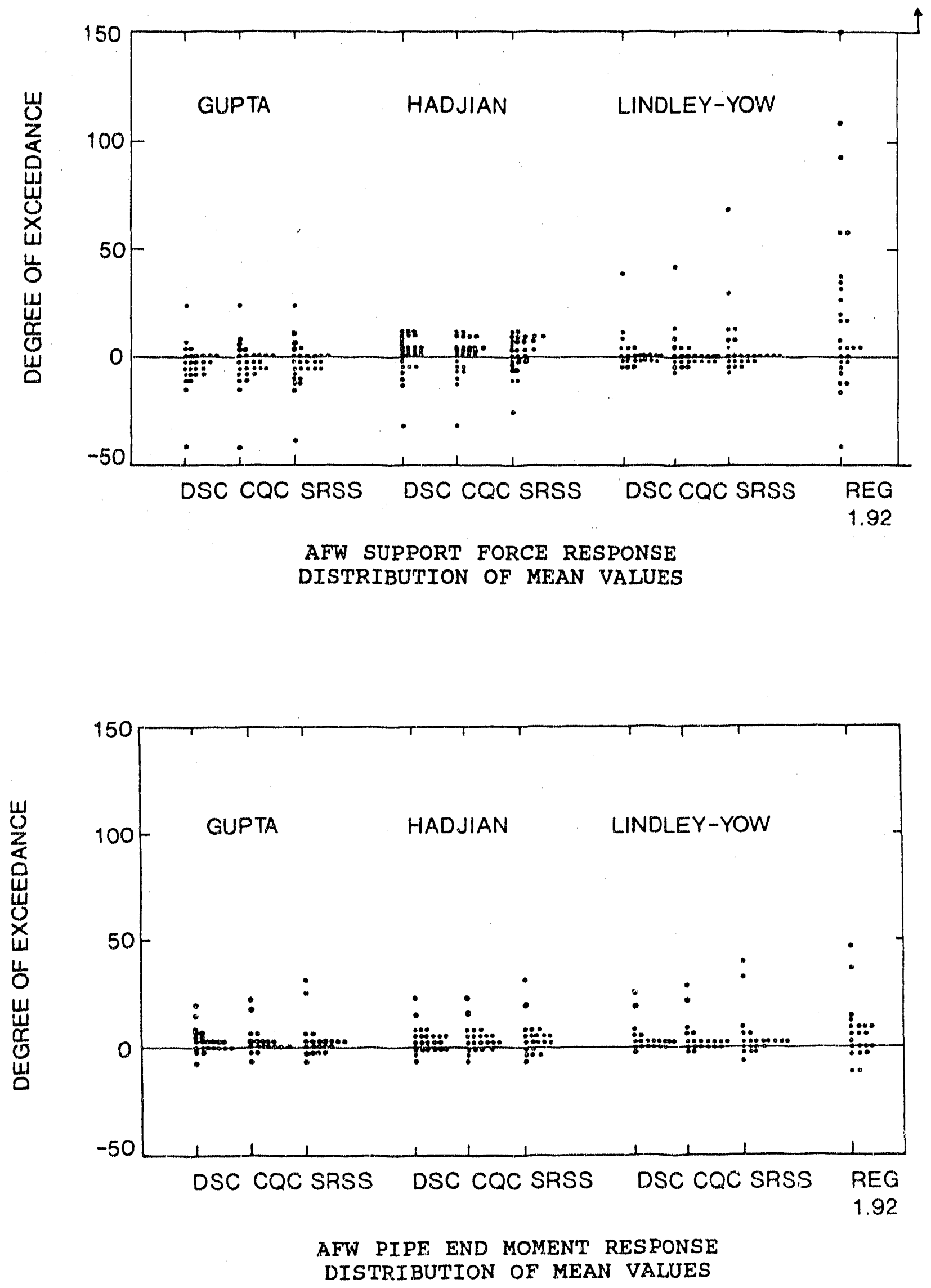

Figure 12 - AFW Problem Statistical Results 


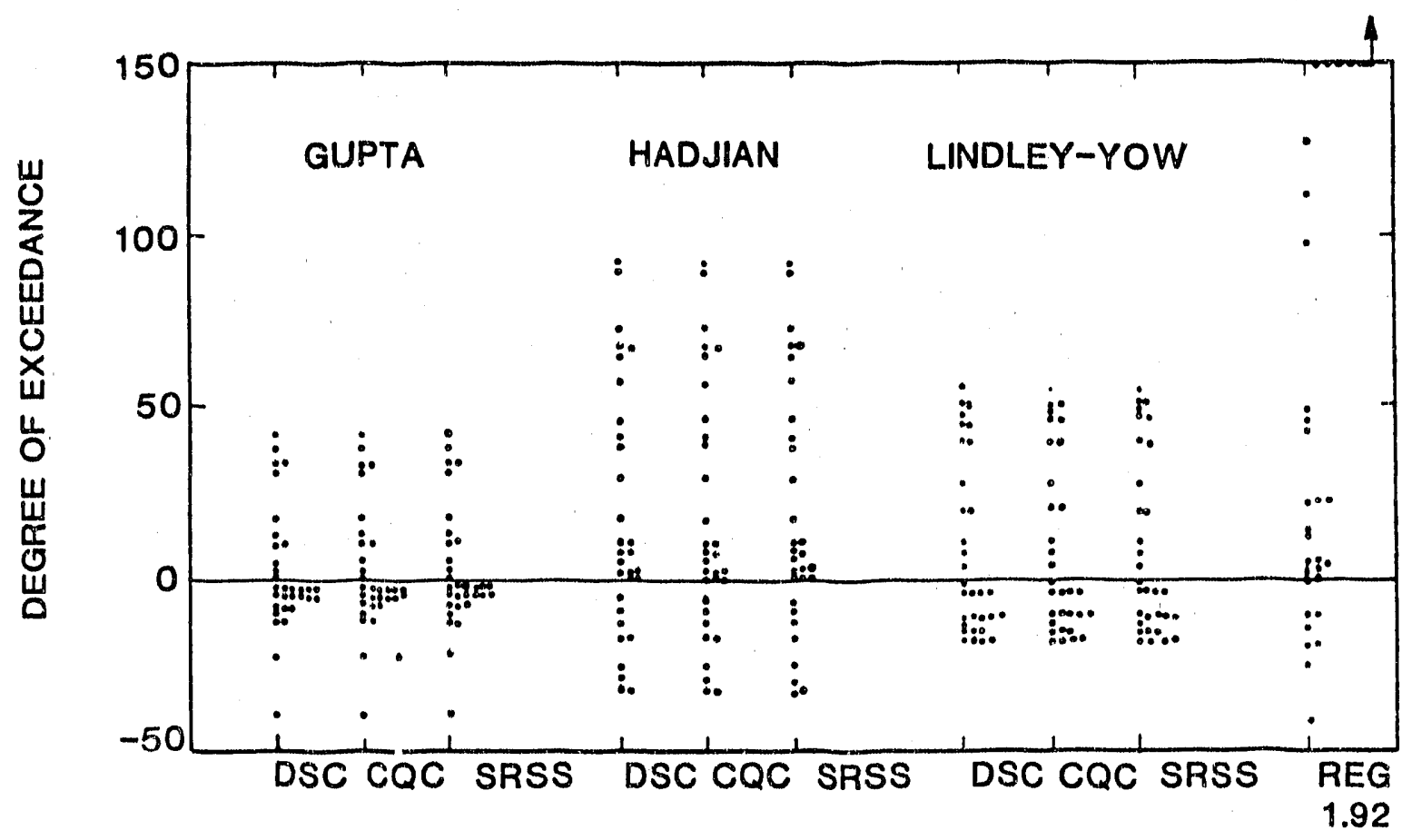

BM2 SUPPORT FORCE RESPONSE

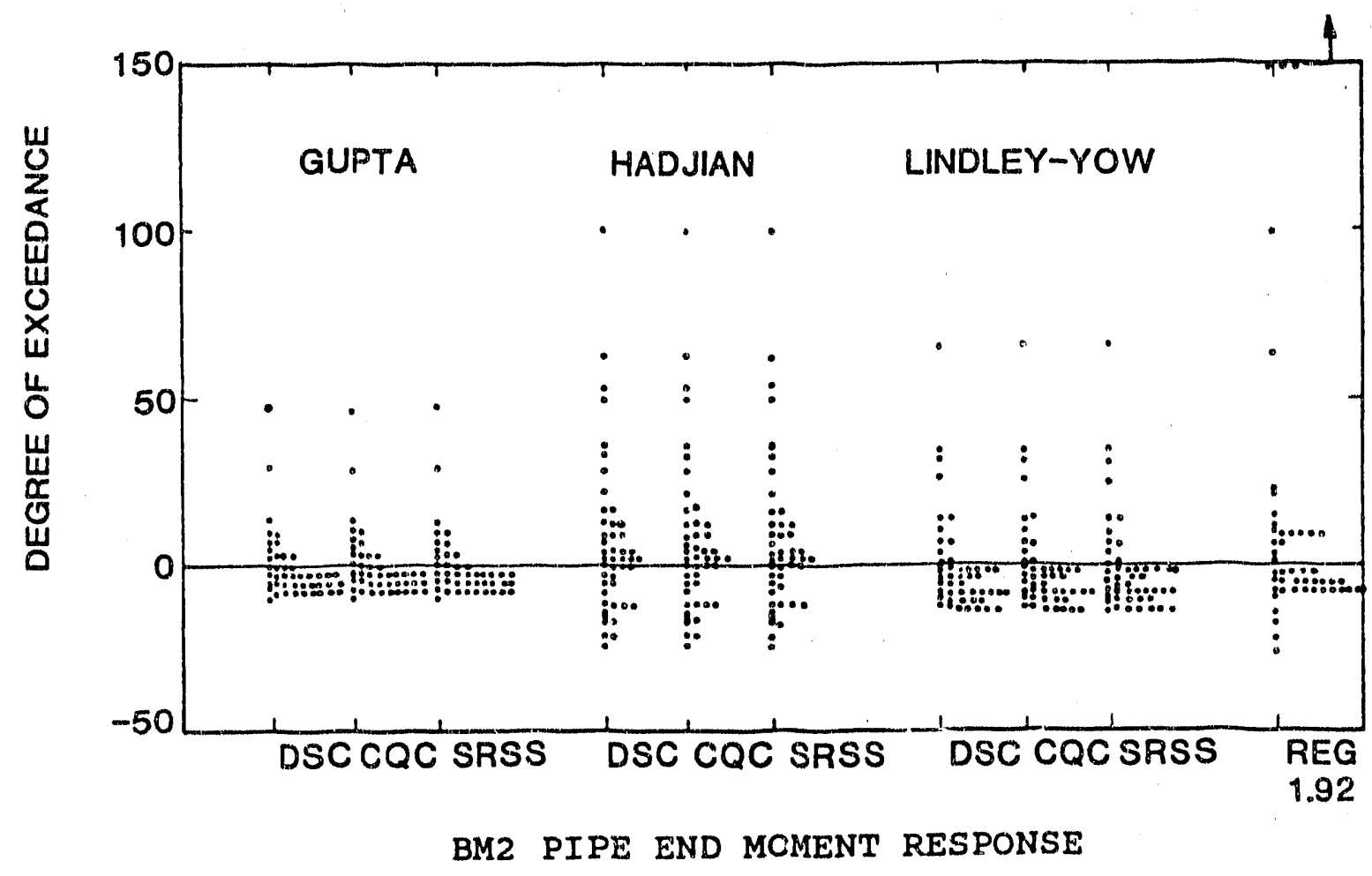

Figure 15 - BM2 Problem Distribution of Results 

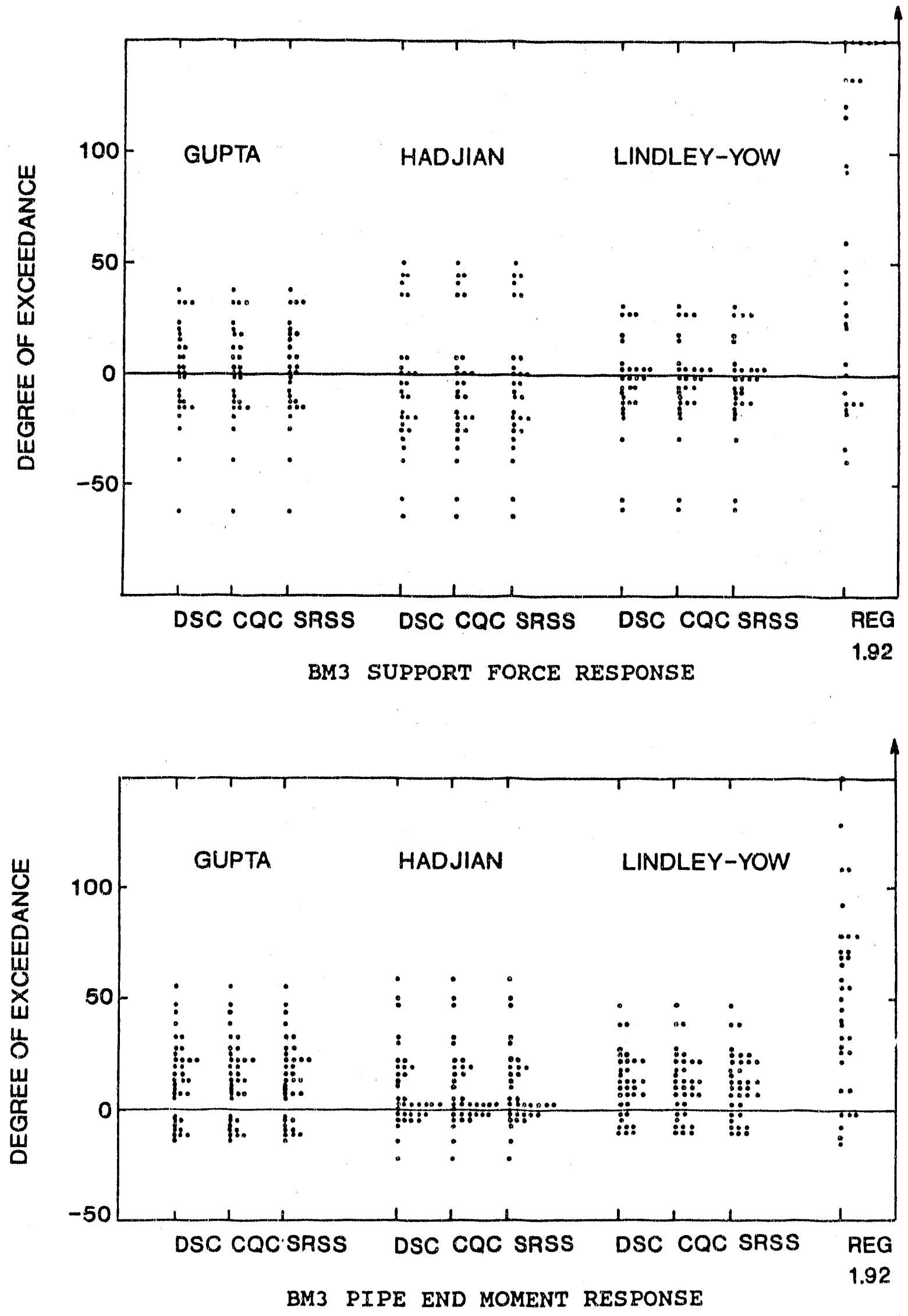

Figure 16 - BM3 Problem Distribution of Results 
The results of the study provide a body of data that can be used to assess the relative merit of each candidate method and the currently accepted method. An ideal method would have small mean error and a small standard deviation about the mean error.

With the exception of the detailed results for each earthquake event for the RHR and AFW problems, all the pertinent study results are depicted in Figures 11 through 16. Since the RHR and AFW results are a statistical set, Figures 11 and 12 can be considered to depict the overall trends to be expected. Figures 13 through 16, on the other hand, show case specific examples of results.

Referring first to Figures 11 and 12 , and accepting the time history estimate of response as the true response, the following observations can be made:

1. Each of the candidate methods can provide underestimates of time history response. The magnitude of the underestimates appears least for the Lindley-Yow procedure and comparable for the Gupta and Hadjian procedures.

2. The currently accepted procedure (REG 1.92) also provides underestimates of time history response. The magnitude of these underestimates are comparable to those exhibited by the candidate methods.

3. The dispersion of the mean value results for the candidate methods is not large. Most results are clustered near the time history response (Degree of Exceedance $=0$ ) and essentially all results are bounded between Degree of Exceedance values of $\pm 25 \%$. The dispersion of results appears least for the Lindley-Yow procedure and comparable for the Gupta and Hadjian procedures.

4. The REG 1.92 procedure shows a greater dispersion of results than the candidate methods. The results range from Degree of Exceedance values of $-25 \%$ to Degree of Exceedance values exceeding $150 \%$, with most results exhibiting positive Degree of Exceedance values.

5. Essentially no variation of results as a function of a method used to combine over periodic modal responses (DSC, CQC and SRSS) are evident.

Observations 3 through 5 can be substantiated by reference to Tables $3 \mathrm{a}, 3 \mathrm{c}, 4 \mathrm{a}$ and $4 \mathrm{c}$. The last rows on these tables list the average and standard deviation of the mean value results for the RHR and AFW problems. As can be seen, the means of the mean value results for the candidate methods are very comparable and show values between -4 and +8 . The corresponding standard deviation 
values are of the order of 10 indicating the results are all tightly clustered. The corresponding values for the REG 1.92 procedure are four to five times larger indicating a higher mean and greater dispersion for this method. Referring again to the mean value listings for the candidate methods only slight differences are evident between the DSC, CQC and SRSS methods.

Referring next to Figures 13 through 16 , the following observations can be made:

1. Greater variability between the results for the candidate methods in a given problem, and between the results for the candidate methods between problems, is evident. On the whole, the results developed with the Iindley-yow method show the most consistency over the problem set.

2. All methods provide underestimates of time history response. For these single seismic event evaluations, underestimates as large as $75 \%$ on the Degree of Exceedance scale are evident.

3. The REG 1.92 procedure shows the greatest dispersion of results, with some response estimates exceeding the time history response by a fartor of four.

4. Essentially no variation of results attributable to the DSC, CQC or SRSS methods is evident.

All these observations are consistent with those noted above for the RHR and AFW problems.

The magnitude of the error associated with the Gupta method can be reduced if a greater effort is given to the development of the modal coefficients $\left(\alpha_{1}\right)$. These coefficients, which quantify the correlation between each modal response and the input excitation, can be computed numerically from the input time history record. The formula for the coefficients given in section 2.1.1 and used in tnis study provide idealized estimates of the coefficients that may not give representative results in individual. cases. The formula was used in this study because it was anticipated that it would be used in most applications of the Gupta method.

The insensitivity of the results to the method used to combine over the periodic modal responses, or the apparent lack of any impact of closely spaced modes, leads to either of the following conclusions. Either, the spacing of modes has no impact on results, or, the problem set was deficient in such a way that this effect was not revealed. Since a large body of evidence exists to support the contention that closely spaced modes impact on response results, the second conclusion is felt to be the case in this study. 
To support this conclusion, an evaluation of the problem set was conducted. In particular, the frequency of occurrence of closely spaced modes within the problem set, their modal order, the magnitude and correspondence of their associated modal participation factors, their frequency magnitude relative to the input forcing spectra and the correspondence between the modal participation factors and the input excitation (X) direction, were investigated. Characteristics that would assure large impact include closely spaced modes at a low frequency, modes that exhibit large modal participation factors in the $\mathrm{x}$ direction, and clustered frequencies occurring in the amplified region of the input spectra.

The following observations were made:

i) For the RHR problem, the frequency of the first set of closely spaced modes occurs at a frequency corresponding to the ZPA or non-amplified region of the input. The associated modal participation factors are dominant in the $\mathrm{Y}$ and $\mathrm{Z}$ directions respectively which are not consistent with each other ox the input excitation. A null impact should be expected.

ii) For the AFW problem, closely spaced modes occur at a low frequency order corresponding to a frequency in the amplified region of the spectra. Associated modal participation factors are $X$ and $Z$ respectively, one corresponding to the input direction but the other not. Small impact should be expected.

iii) For the $z$ bend, their are no clustered modes and consequentially a null impact should be expected.

iv) For the BM1, BM2 and BM3 problems, clustering occurs for higher order modes at frequencies corresponding to the ZPA region of the input and with associated modal participation factors that do not correspond with each other or the input. A null impact should be expected.

A review of the data set shows that only for the AFW problem was there any indication of an influence of closely spaced modes. This is consistent with the observations above and confirms the conclusion that the problem set was deficient in characteristics that make an investigation of alternate methods to address closely spaced modes viable. Unfortunately, in order to make full use of existing data, the problem set was prescribed at the outset of the study precluding any evaluation or optimization of it for the purpose of this study. 


\section{CONCLUSIONS}

The Gupta, Hadjian and Lindley-Yow methods are shown to address frequency correlation effects rationally. All of these methods provide results that exhibit smaller mean error and standard deviation than the procedure currently accepted by the NRC. Regarding alternate methods to combine modes with closely spaced frequencies the study results were inconclusive. How .uver there is adequate information in the literature showing tnat closely spaced modes can have a significant impact on results. Both the Rosenblueth Double sum Method (DSC) and the complete Quadratic Sum Method (CQC) are considered to address the phenomenon more correctly than the simple procedure currently accepted. This is clearly demonstrated in the study performed by Maison, Neuss and Kasai [19], a description of which is presented in Appendix II. Therefore the use of the Gupta, Hadjian or Lindley-Yow methods to address frequency effects in conjunction with either the DSC or CQC methods for modes with closely spaced frequencies should be considered desirable alternates to the methods currently accepted by the NRC.

It is important that any modal analysis technique, including the response spectrum and the time history method, account for the residual rigid response. The residual rigid response was omitted fror the present calculations because it did not directly affect the comparison between the time history and the response spectrum method. Also discussed earlier in the report, in the response spectrum method and in the combination of modal responses, it is necessary to retain the unique signs of modal responses $\left(R_{1}\right)$. For example, this uniqueness will be destroyed if an absolute sign is applied to the participation factor as it is done in some computer programs.

As noted in the Introduction, the response spectrum method is a design method and is not expected to give results that are in total agreement with the time history results. On the average, the errors between the response spectrum and the time history results are likely to be quite small, tencing to zero as the number of ground motions considered in the analysis is increased. This will be true only when the modal combination methods are rational and applied correctly. Understanding of this concept is important when reviewing the results from the present study. There are many response spectrum calculated results that are less than (and others that are more than) those from the corresponding time history results. That does rut make the response spectrum method unconservative (or conservative). For design purposes, a rationally formulated response spectrum analysis is superior to a single time history analysis.

To put things in perspective, there are many other sources of uncertainty and conservatism in the seismic design of structures. These are, for example, a low probability design earthquake event 
and the corresponding definition of parameters (such as peak ground acceleration), use of a mean plus one standard deviation spectral shape (84.1\% probability of nonexceedance), peak broadening of floor response spectra, etc. All of the above assure that the likelihood of exceeding the calculated seismic stresses during an actual seismic event is extremely small. None of them assure that the calculated stresses will never be exceeded. 
8. REFERENCES

1. U... Nuclear Regulatory Commission, "Report of the U.S. Nuclear Regulatory Commission Piping Review Committee," NUREG1061, April 1985.

2. Bezler, P., Wang, Y.K., Reich, M., "Response Margins Investigation of Piping Dynamic Analyses Using the Independent Support Motion Method and PVRC Damping," NUREG/CR-5.105, March 1988.

3. U.S. Nuclear Regulatory Commission, "Dynamic Testing and Analysis of Systems, Components and Equipment," standard Review Plan, NUREG-0800, section 3.9.2.

4. U.S. Nuclear Regulatory Commission, "Combining Model Responses and spatial Components in seismic Response Analysis," Regulatory Guide 1.92, Rev. i, February 1976.

5. Lawrence Livermore Laboratory, "Recommended Revisions to Nuclear Regulatory Commission Seismic Design Criteria," NUREG/CR-1161, June 1979.

6. Gupta, A.K., Response Spectrum Method, Blackwell, 1990.

7. Hadjian, A.H., "Seismic Response of Structures by the Response Spectrum Method," Nuclear Engineering and Design, Vol. 66, No. 2. August, 1981.

8. Lindley, D.W., and Yow, J.R., "Modal Response Summation for Seismic Qualification," Proceedings 2nd ASCE Conference on Civil Engineering and Nuclear Power, Knoxville, Tennessee, september 1980 .

9. Kennedy, R.P., "Position Paper on Response Combinations," SMA 1.2211.02-R2-0, March 1984.

10. Rosenblueth, E., and Elorduy, J., "Responses of Linear Systems to Certain Transient Disturbances," Proceedings 4 th World Conference on Earthquake Engineering, Santiago, Chile, 1969.

11. Wilson, E.L., Der Kiureghian, A. and Bayo, E.P., "A Replacement for the SRSS Method in Seismic Analysis," Earthquake Engineering and Structural Dynamics, Vol. 9, 1981, pp. 187192.

12. American Society of Civil Engineers, "Sejsmic Analysis of Safety Related Nuclear structures and Commentary on standard for Seismic Analysis of Safety Related Nuclear structures," ASCE Standard 4-86, september 1986 . 
13. Nau, J.M. and Hall, W.J., "An Evaluation of Scaling Methods for Earthquake Response Spectra," Structural Research Series No. 499, Department of Civil Engineering, University of Illinois at Urbana-Champaign, Urbana, Illinois, May 1982.

14. Subudhi, M. Bezler, P., Wang, Y.K. and Alforque, R., "Alternate Procedures for the Seismic Analysis of Multiple Supported Piping Systems," NUREG/CR-3811, August 1984.

15. Bohn, M. et al., "Application of the SSMRP Methodology to the Seismic Risk at the Zion Nuclear Power Plant," NUREG/CR-342.9, January 1984 .

16. Chuang, T.Y., et al., "Impact of Changes in Damping and Spectrum Peak Broadening on the Seismic Response of Piping Systems," NUREG/CR-3526, March 1984.

17. Johnson, J.J., et al., "Response Margins of the Dynamic Analysis of Piping Systems," NUREG/CR-3996, September 1984.

18. Johnson, J.J. and Benda, B.J., "Quantification of Margins in Piping System Seismic Response Methodologies and Damping," NUREG/CR-5073, February 1988.

19. Maison, B.F., Neuss, C.F., and Kasai, K., "The Comparative Performance of Seismic Response spectrum Combination Rules in Building Analysis," Earthquake Engineering and structural Dynamics, Vol. 11, 1983, pp. 623-647. 
APPENDIX I

$$
A-i
$$




\section{APPENDIX I}

\section{Degree of Exceedance Result Tables}

Page

o Table Descriptors....................... A A

o RHR Model............................. A-2

o AFW Model............................ A-68

$\quad z$-Bend Model.......................... A

$\quad$ BM1 Model............................. A -137

$\quad$ BM2 Model............................. $A-140$

○ вмз Model............................ A-143 


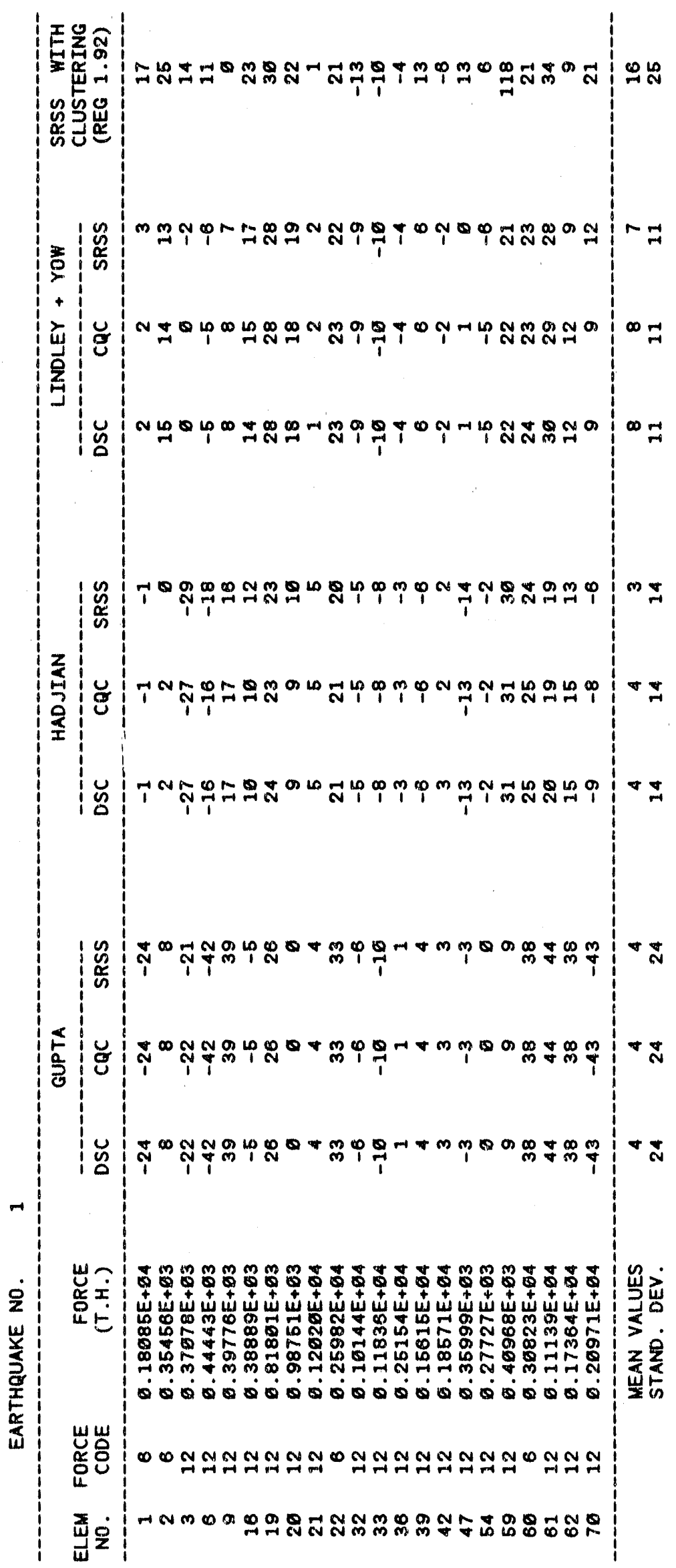




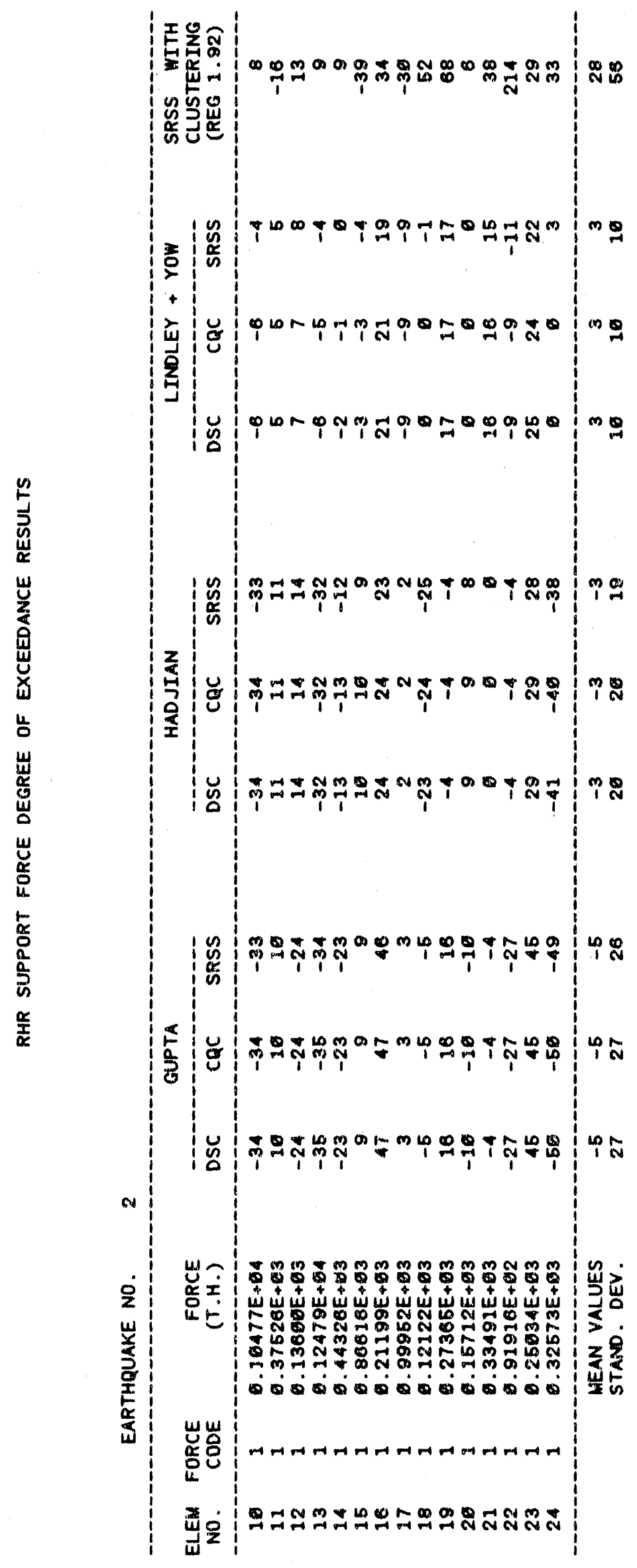

$$
\text { A-4 }
$$


Abbreviations and Symbols

TH

RS

Degree of Exceedance

DSC

CQC

SRSS

REG 1.92

Gupta

Hadjian

Inindley-Yow
Description

Time History Data

Response spectrum Estimate of Result

Degree of Exceedance = $100(\mathrm{RS}-\mathrm{TH}) / \mathrm{TH}$

Rosenblueth Double Sum Method for combining modal responses.

Complete Quadratic Combination Method for Combining Modal Responses.

Square root of sum of square Method for Combining Modal Responses.

NRC Accepted Grouping Method for Combining Modal Responses.

Gupta Method to account for frequency dependent correlation effects.

Hadjian Method to account for frequency dependent correlation effects.

Lindley-low Method to account for frequency dependent correlation effects. 


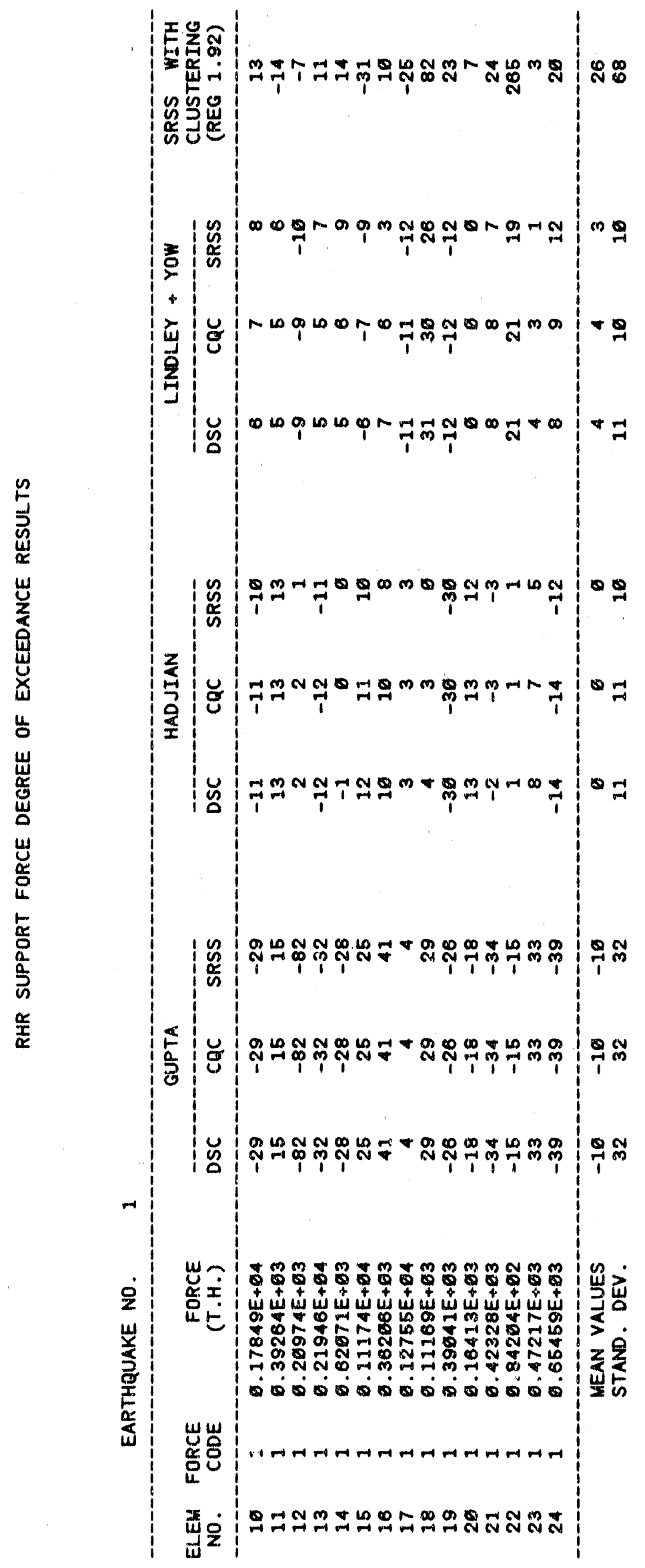




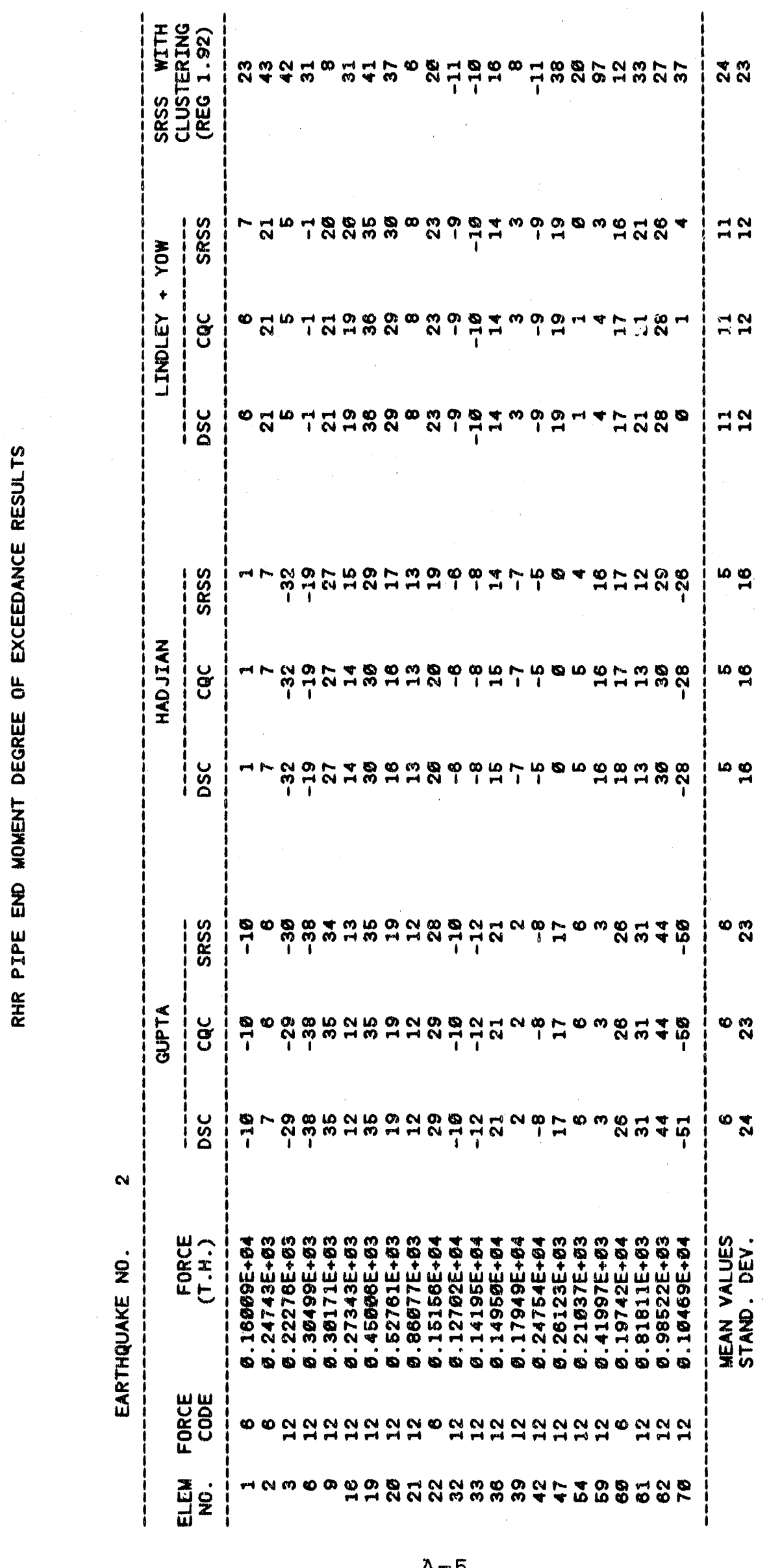




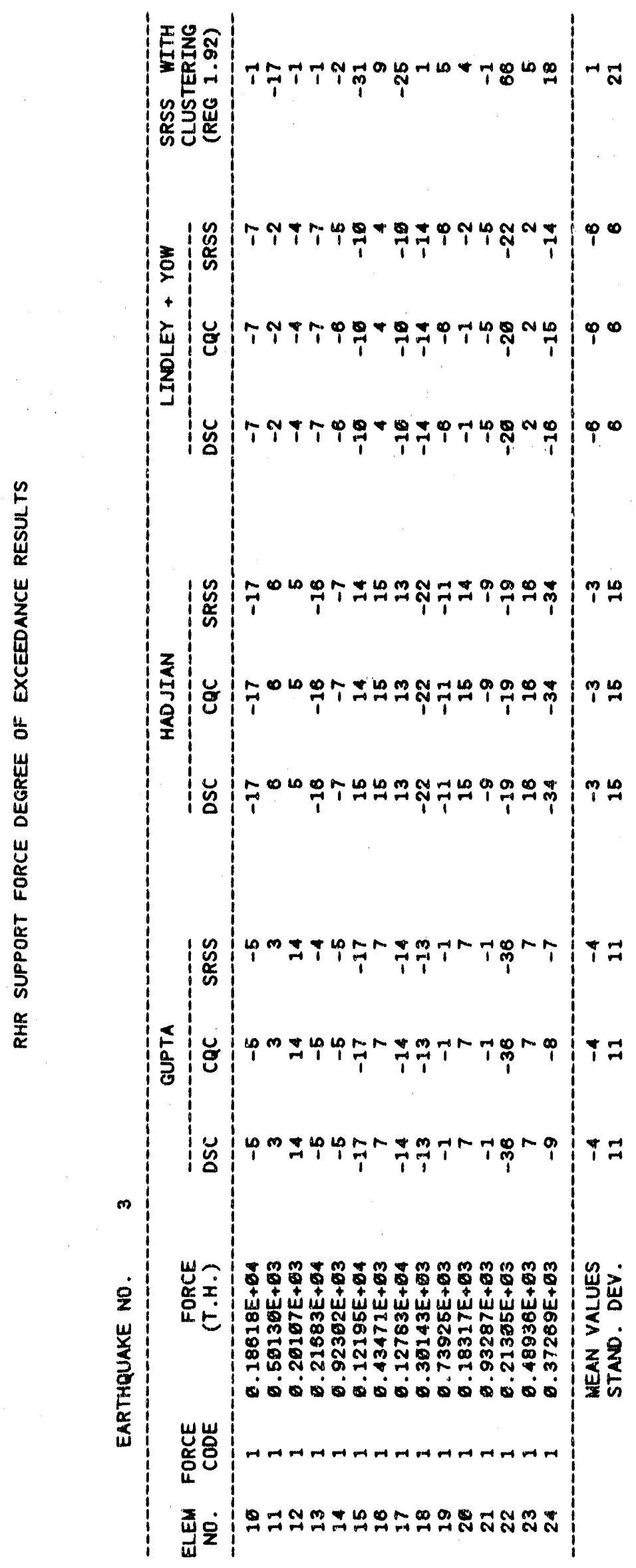

A- 6 


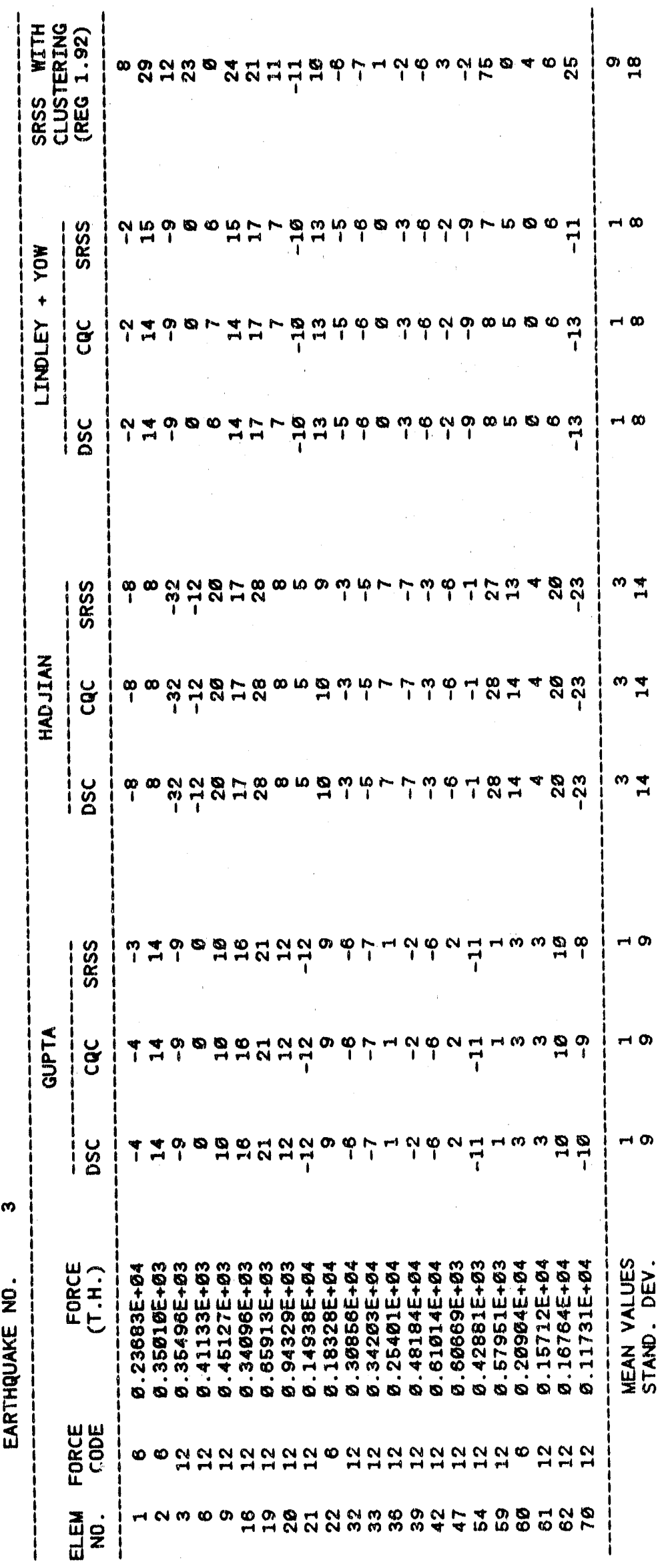




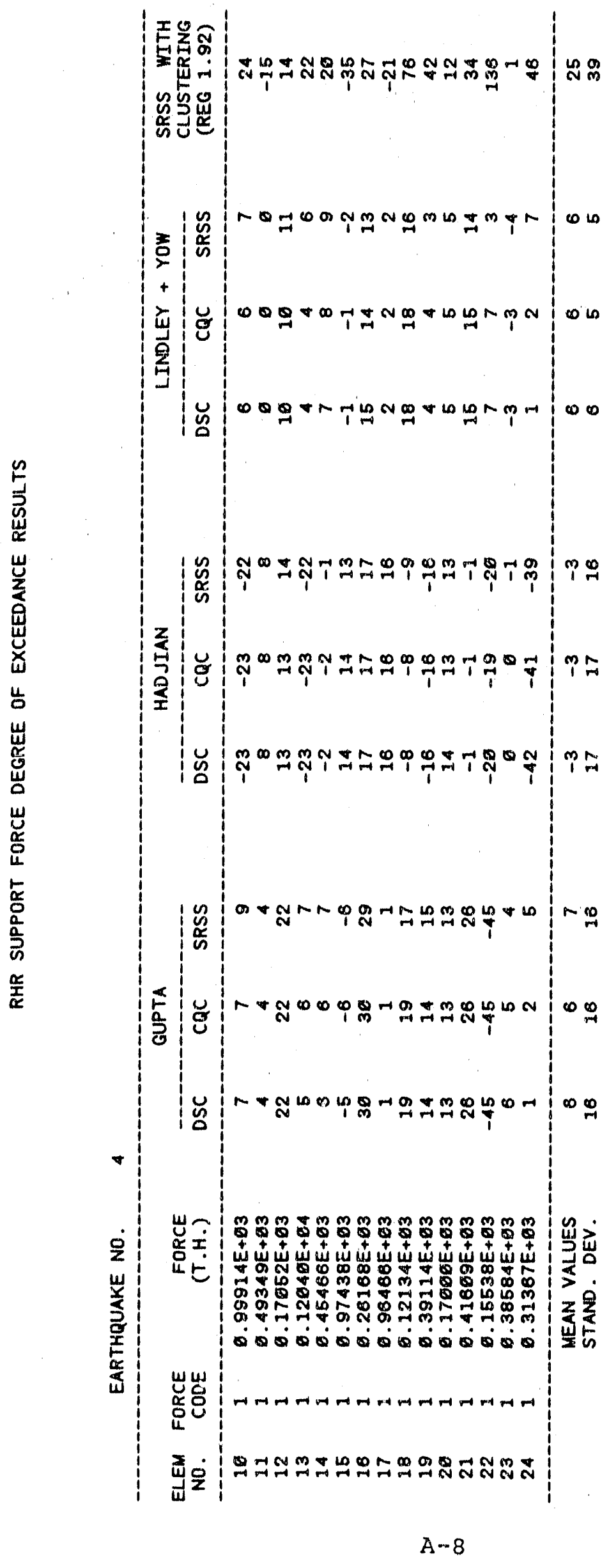




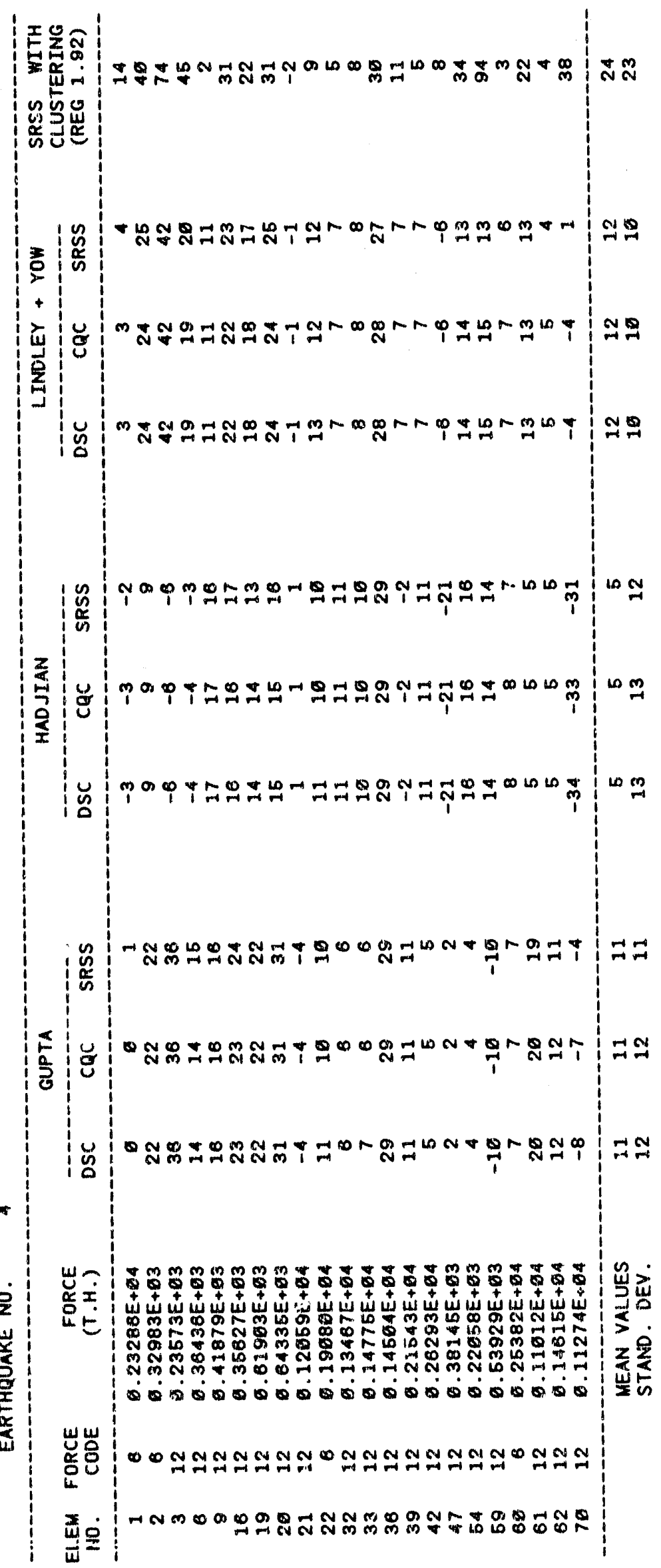

$$
\text { A }-9
$$




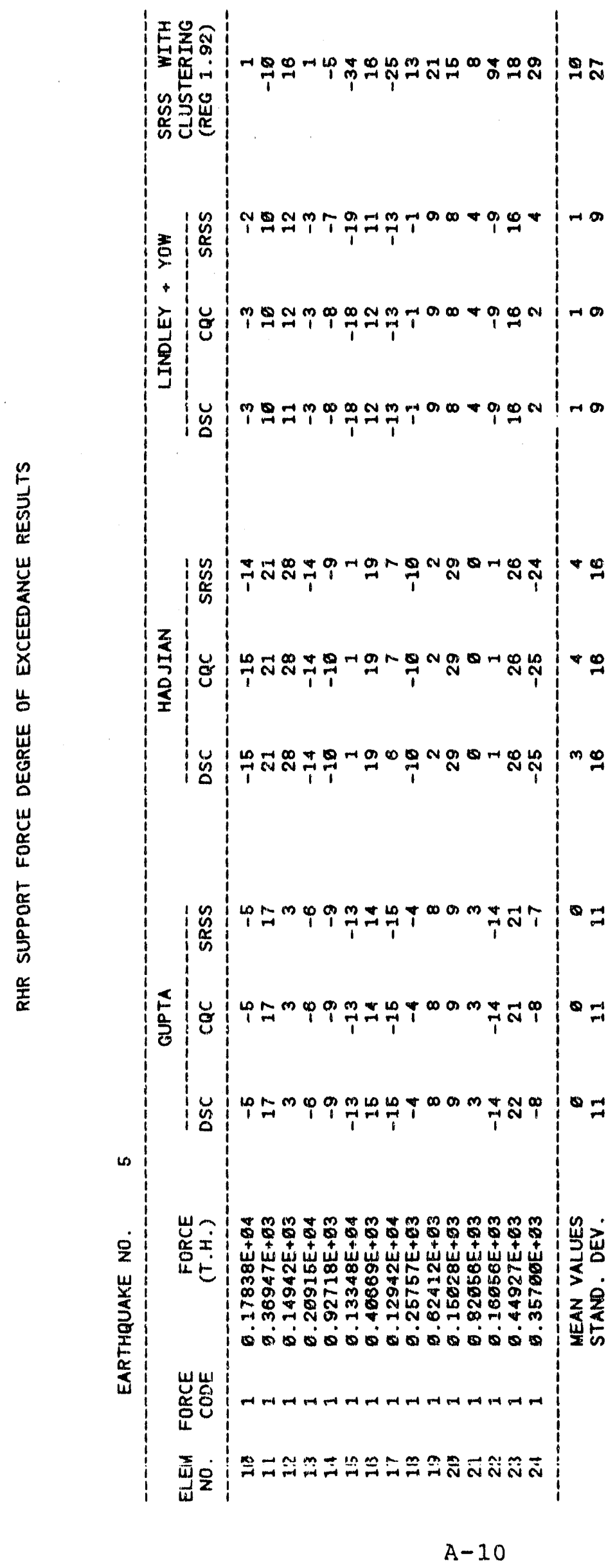




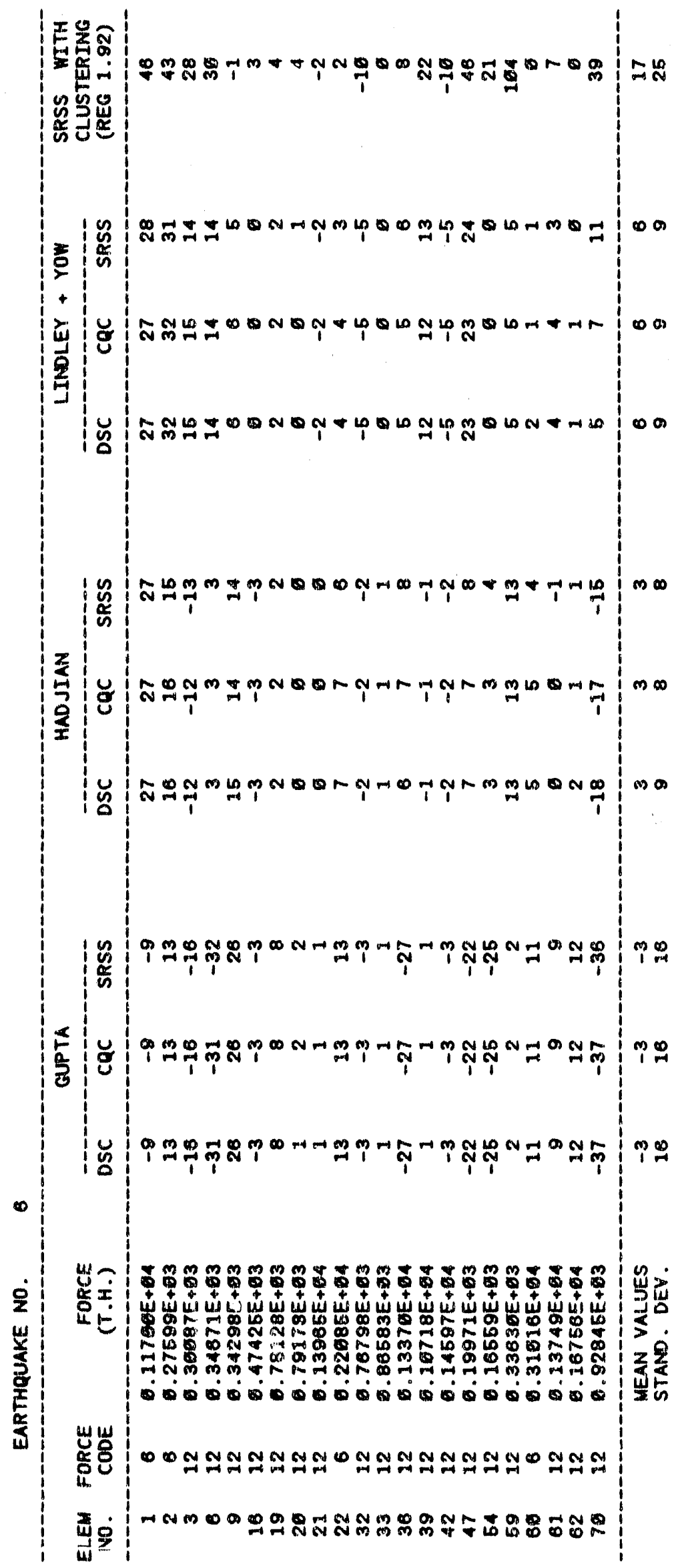

A-13 


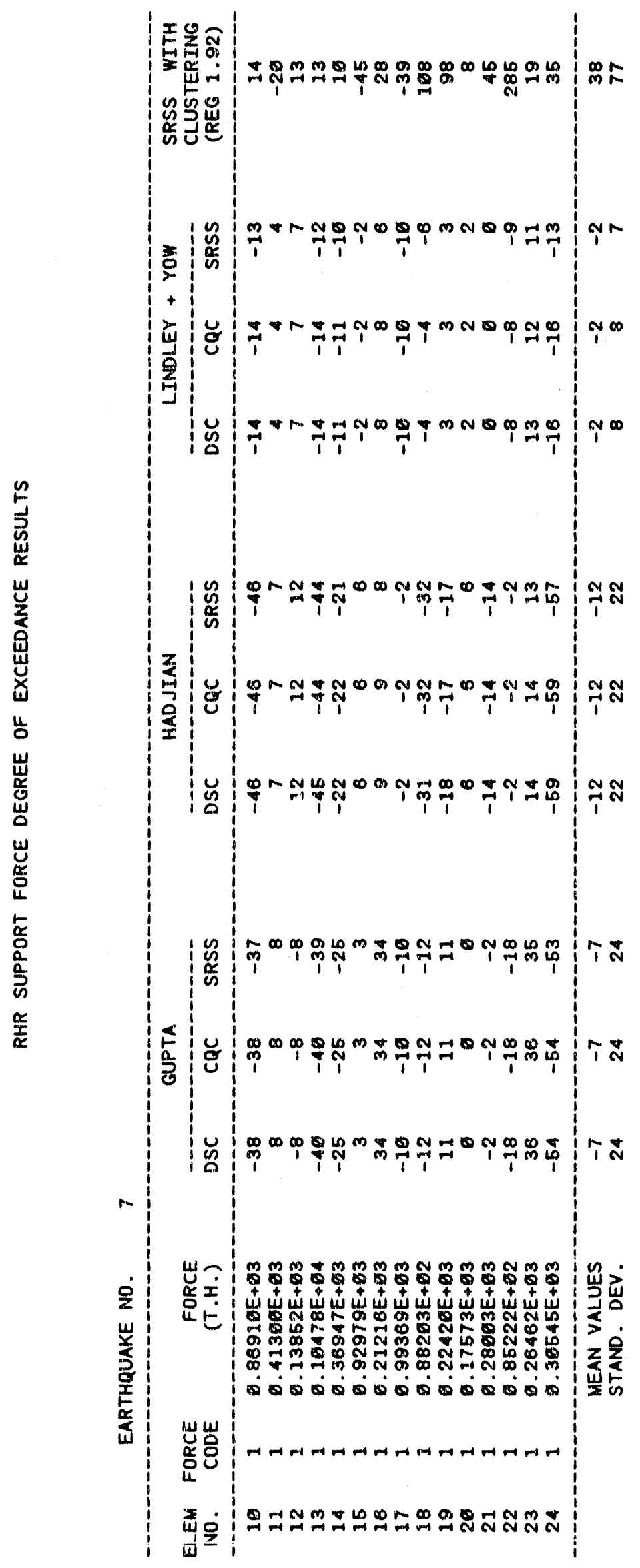

$A-14$ 


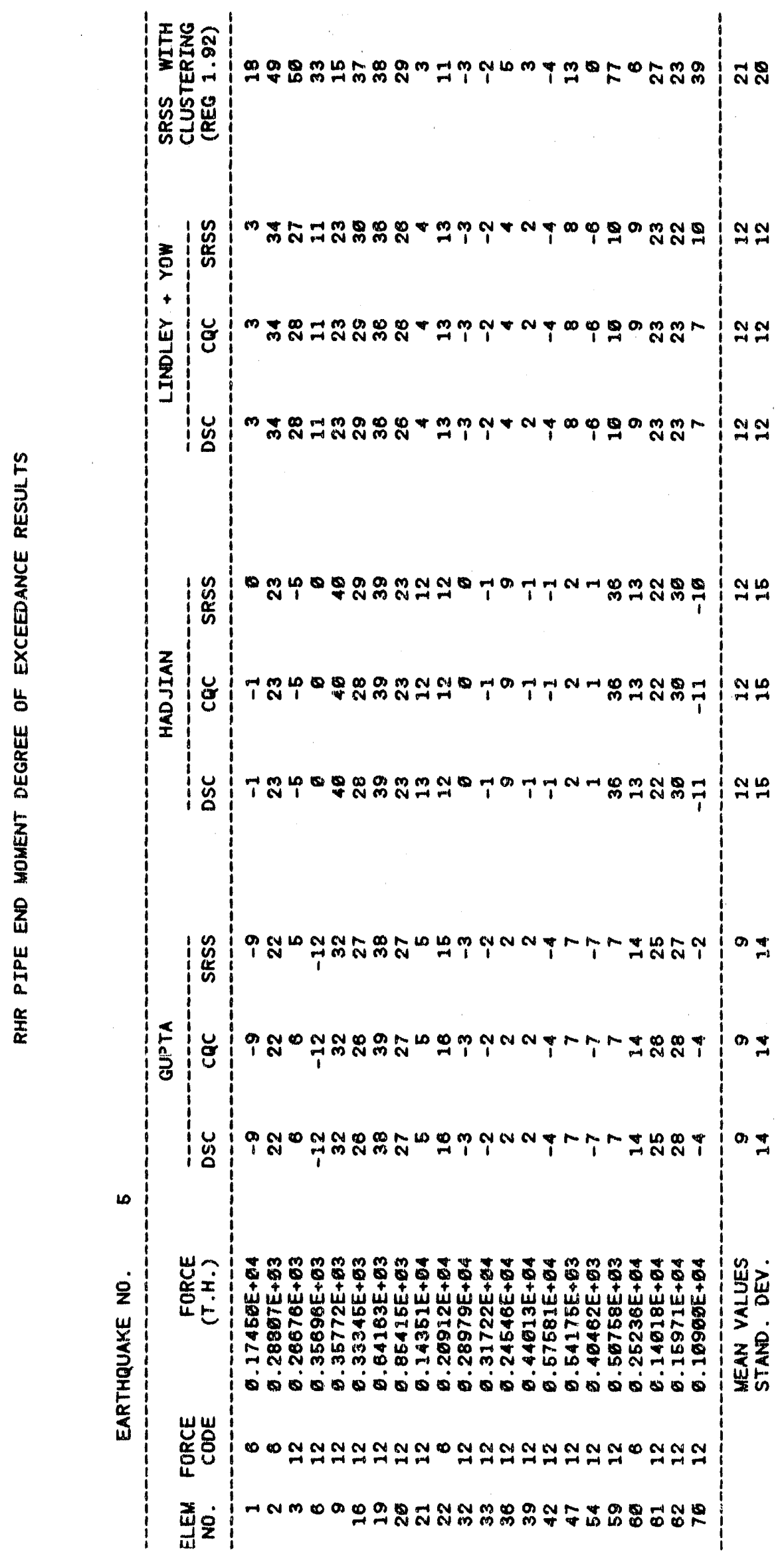




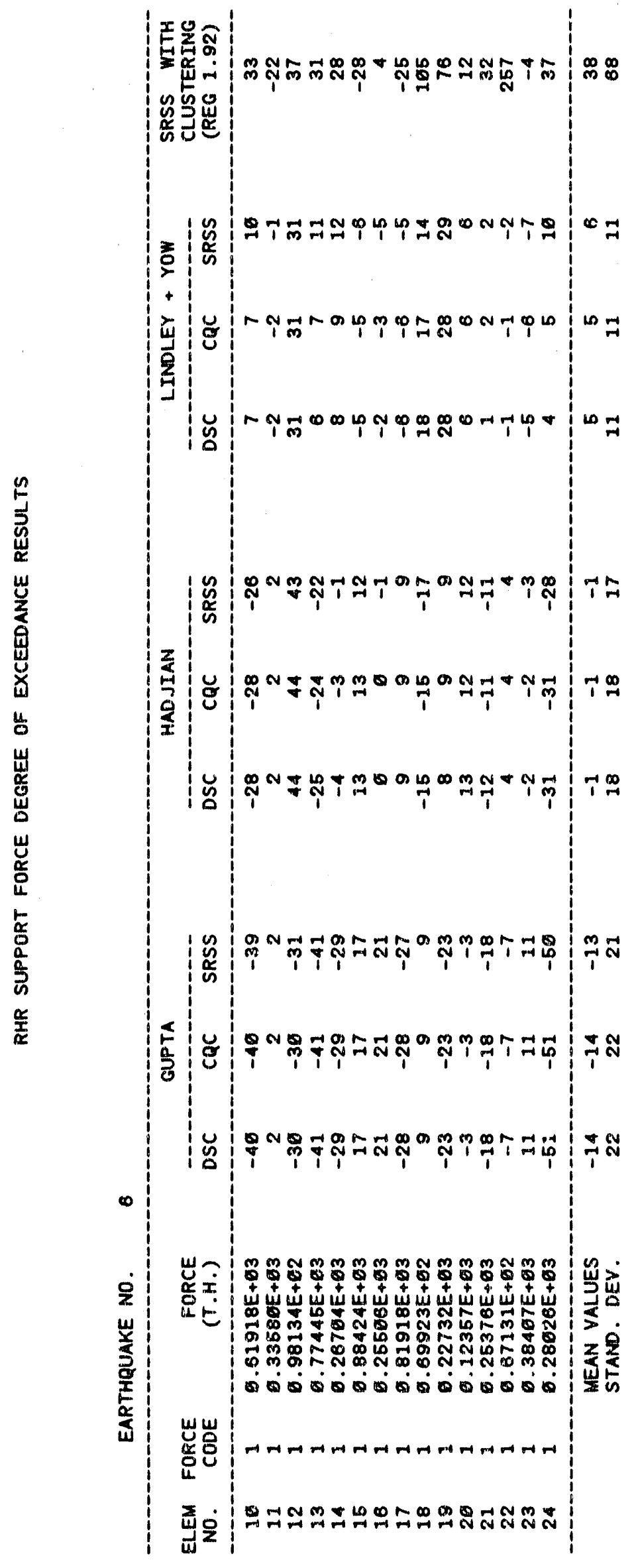

A -12 


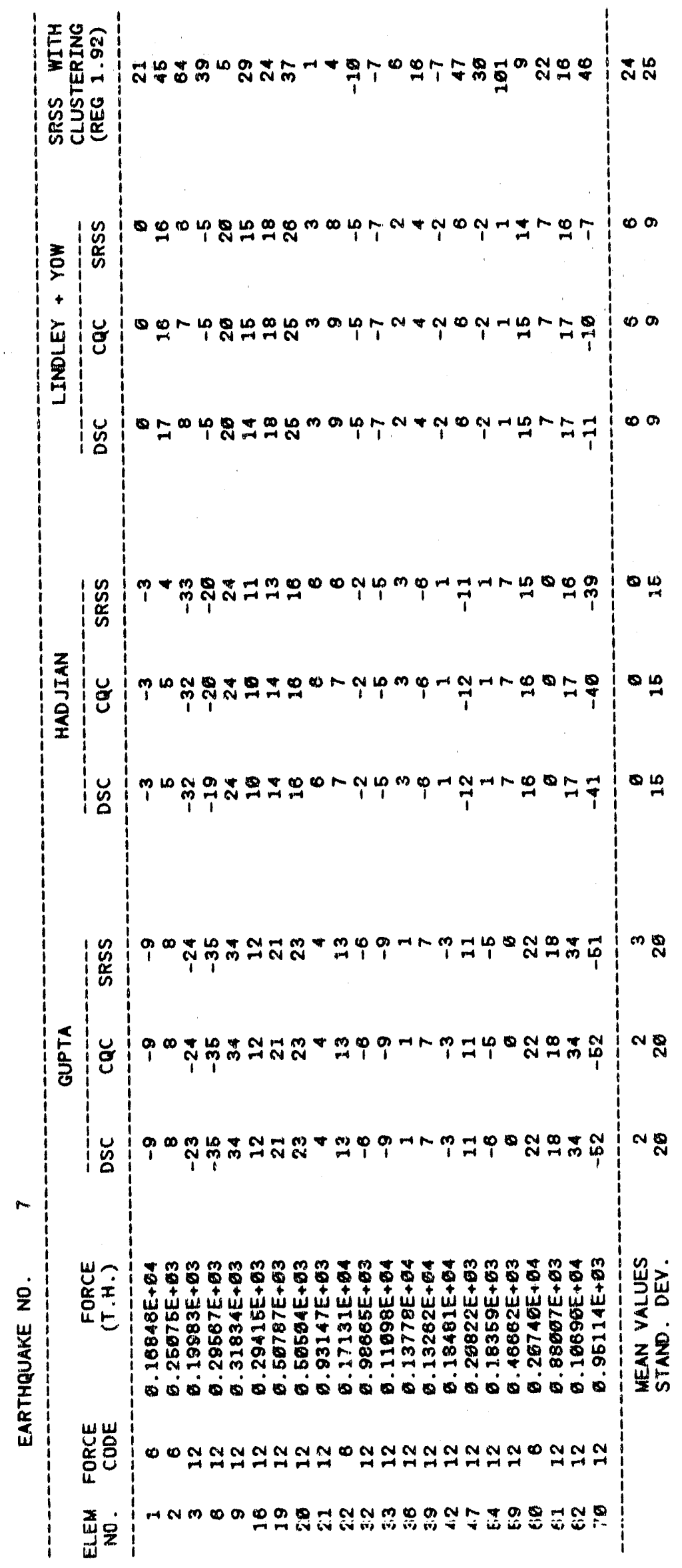

A -15 


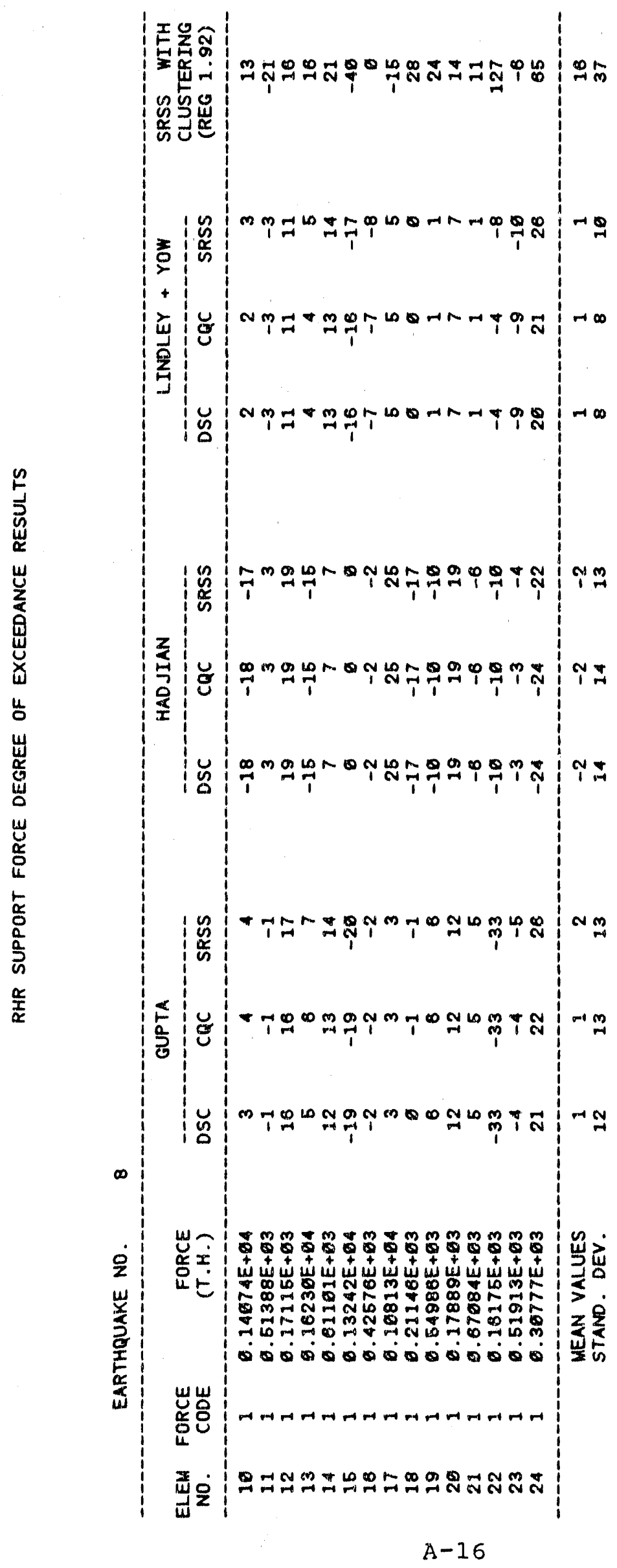




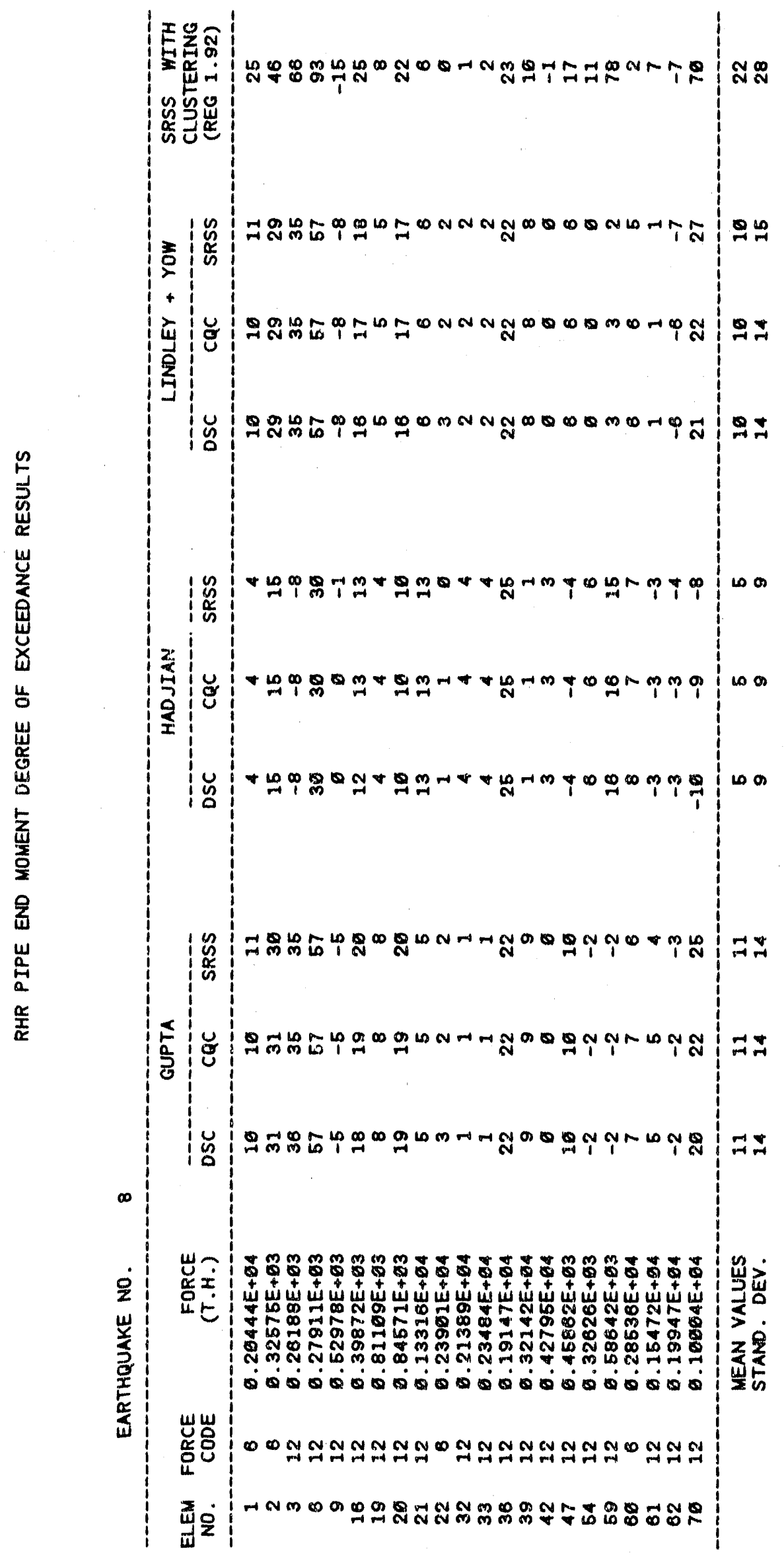

$A-17$ 


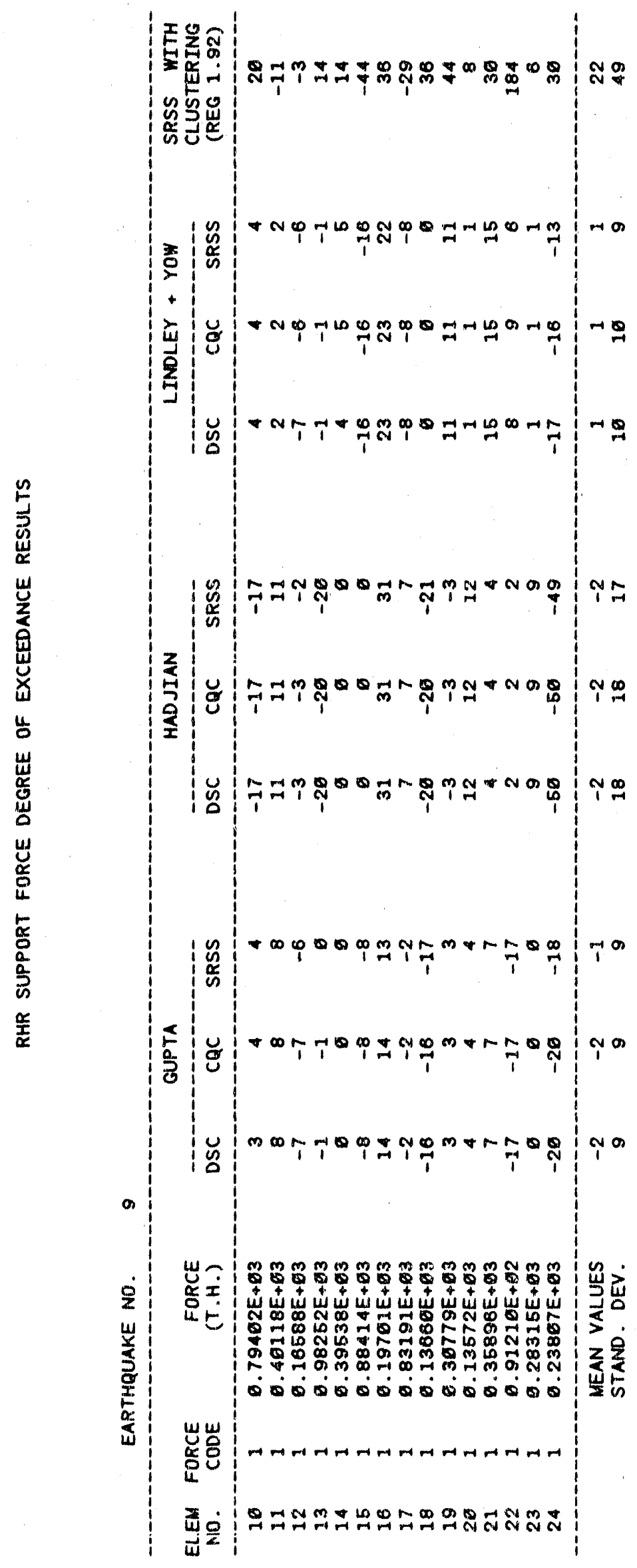

$A-18$ 


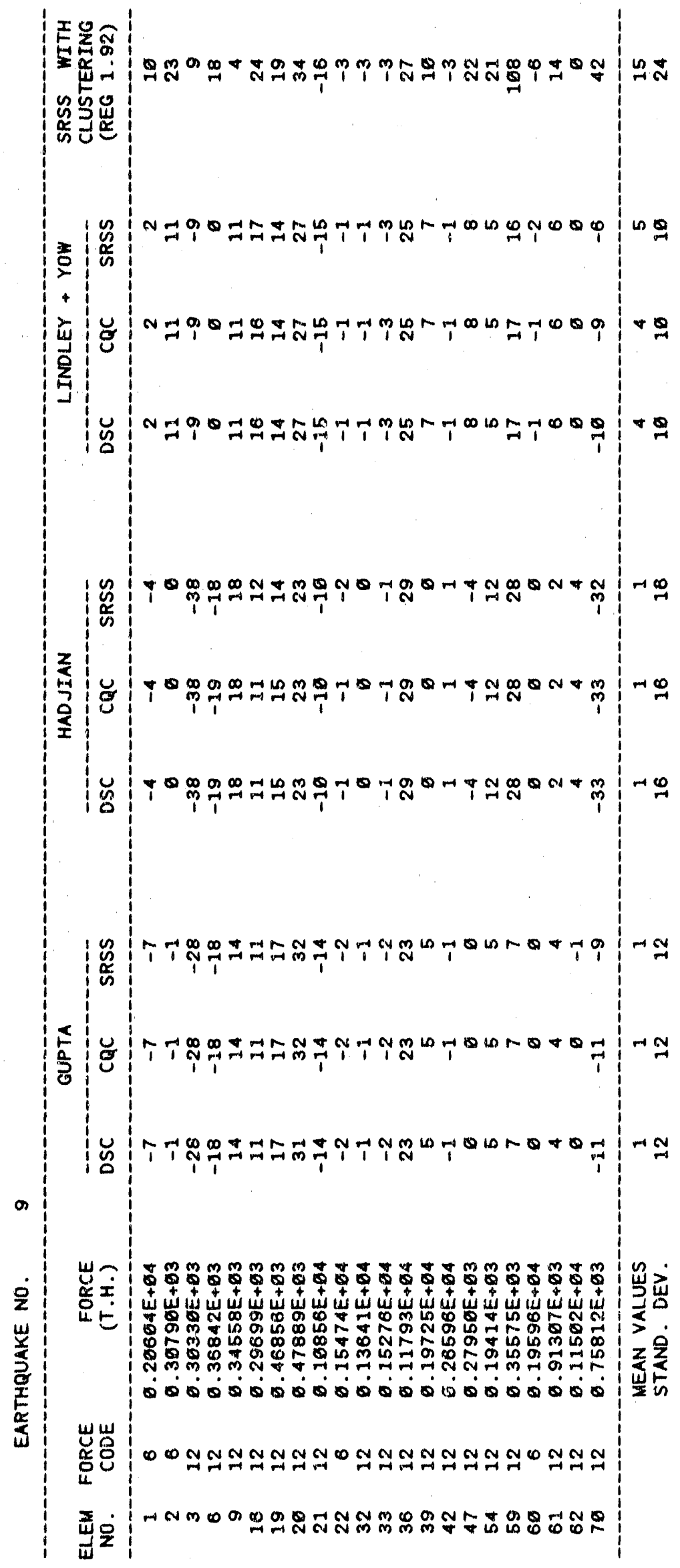

$$
\text { A-19 }
$$




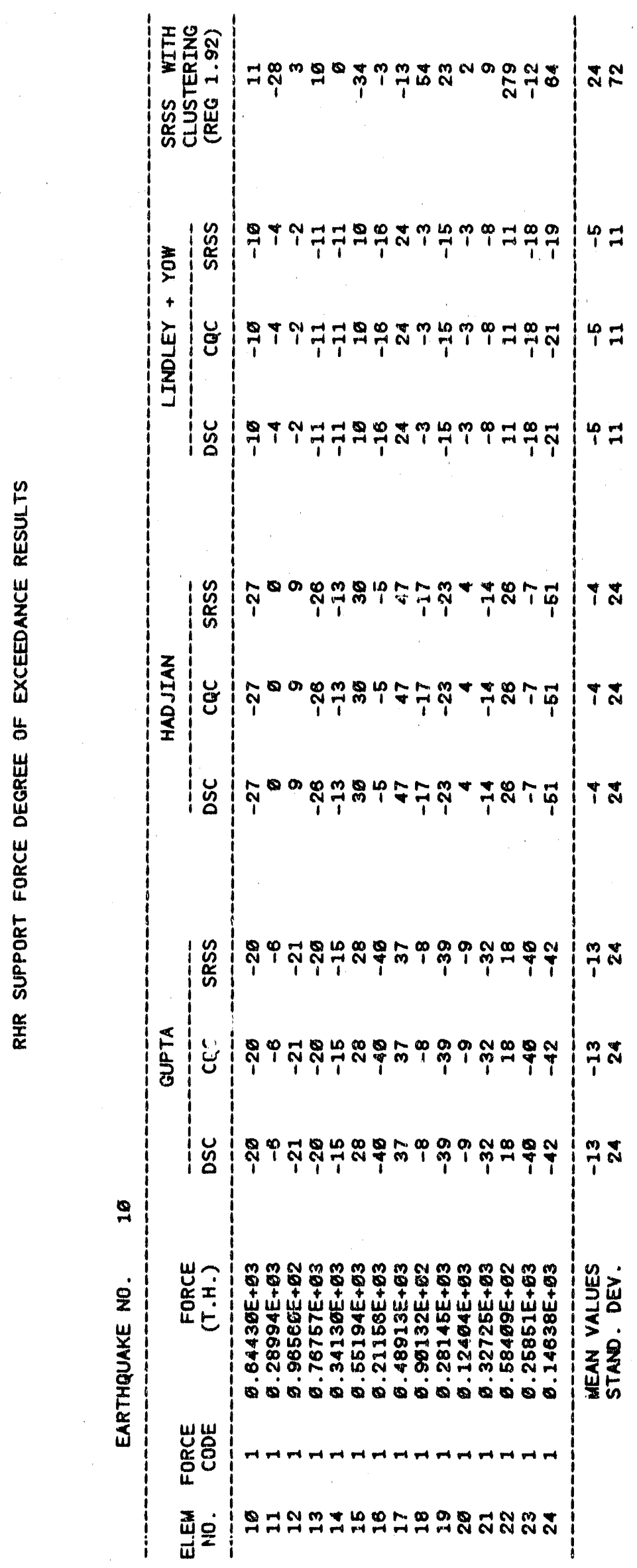

$A-20$ 


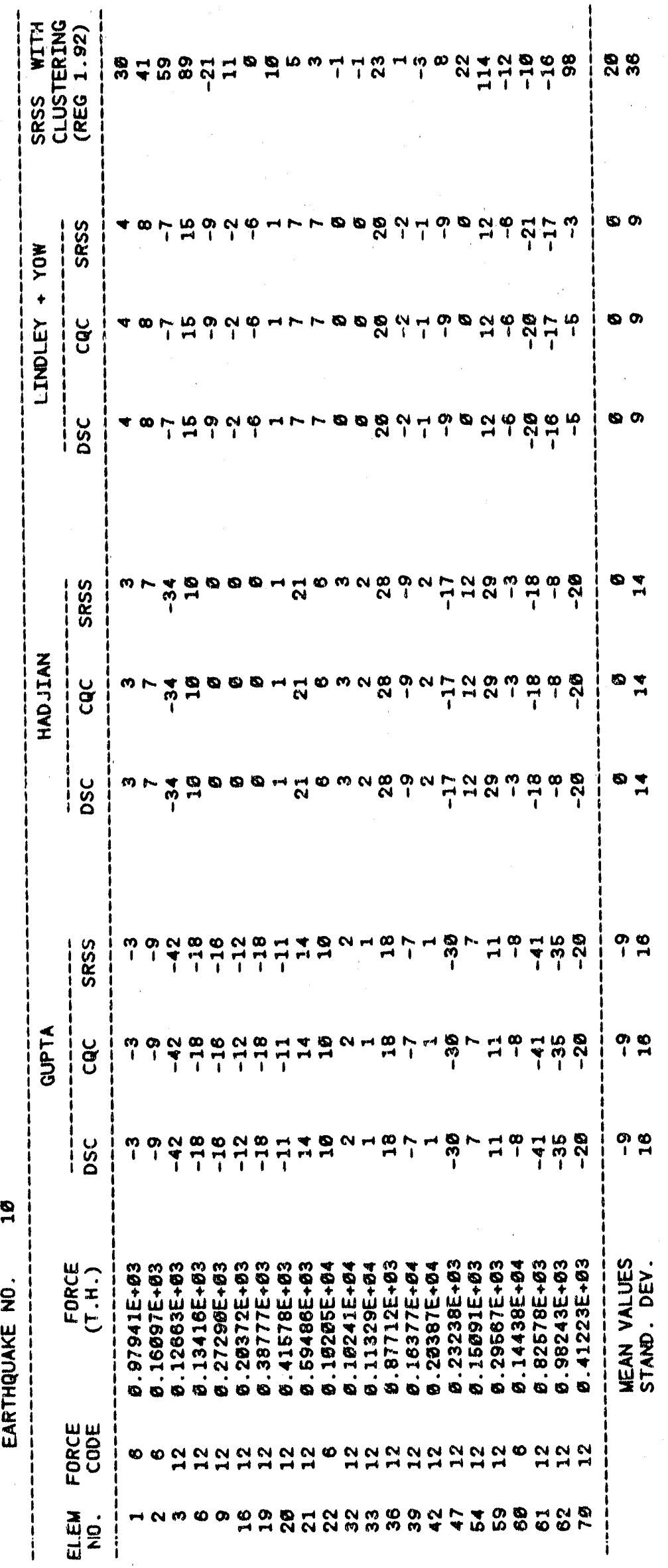

$$
A-21
$$




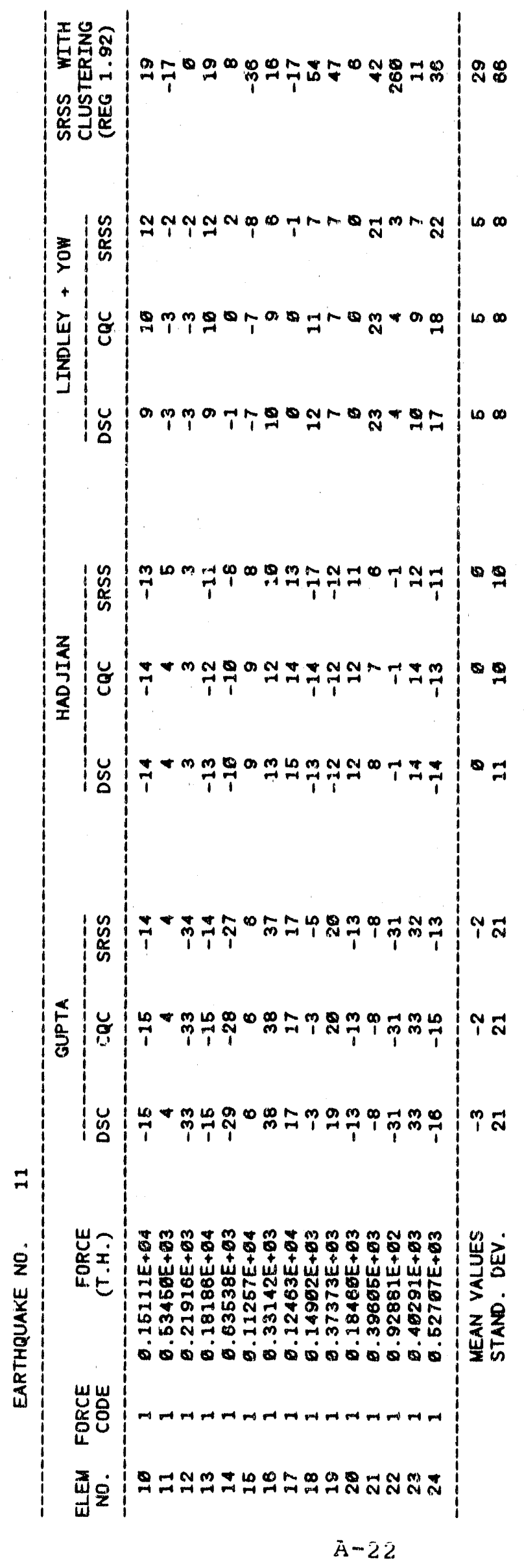




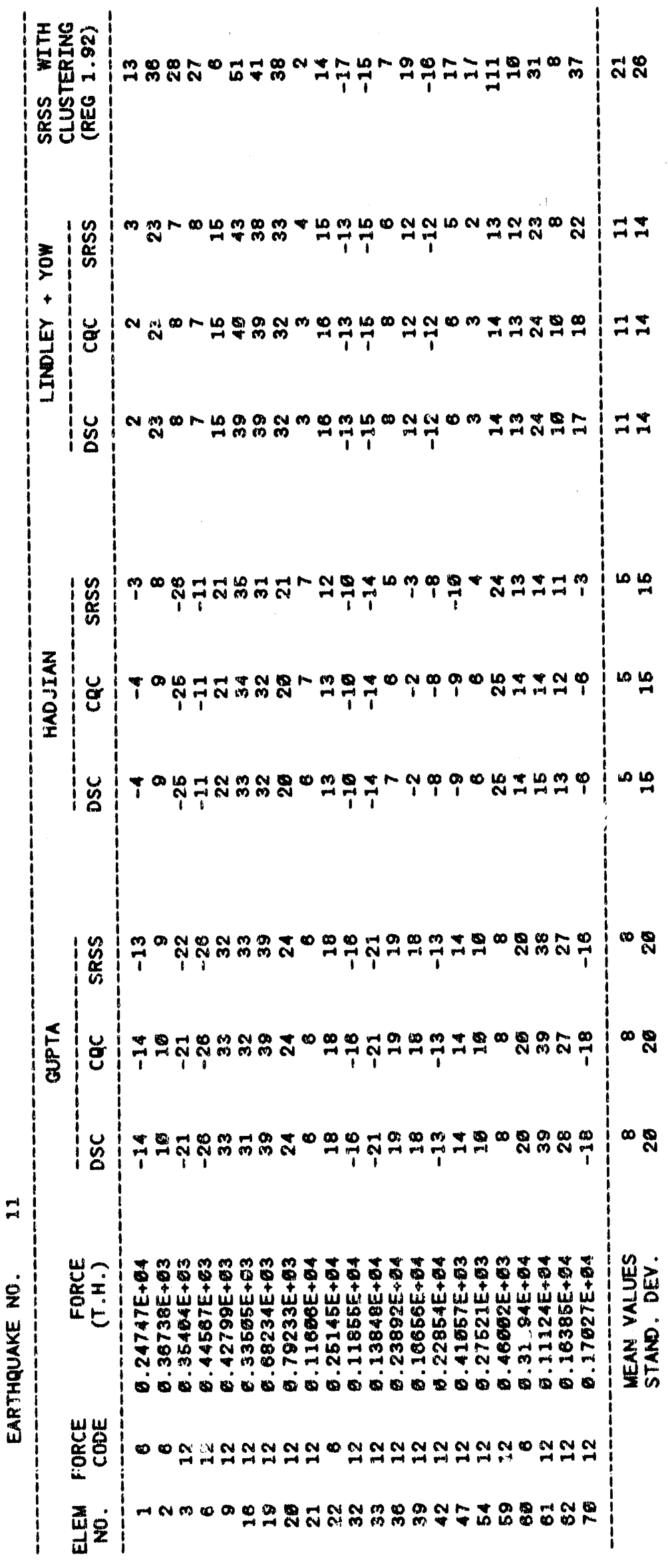

$$
A-23
$$




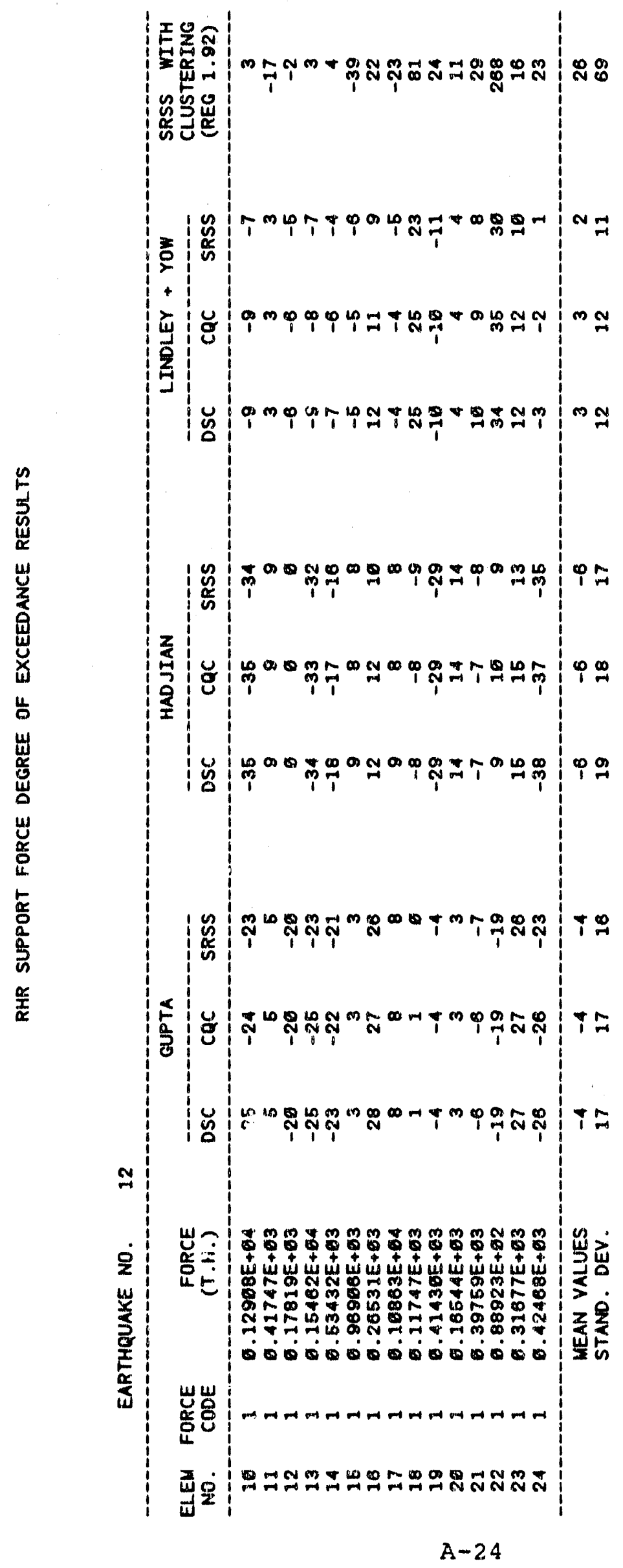




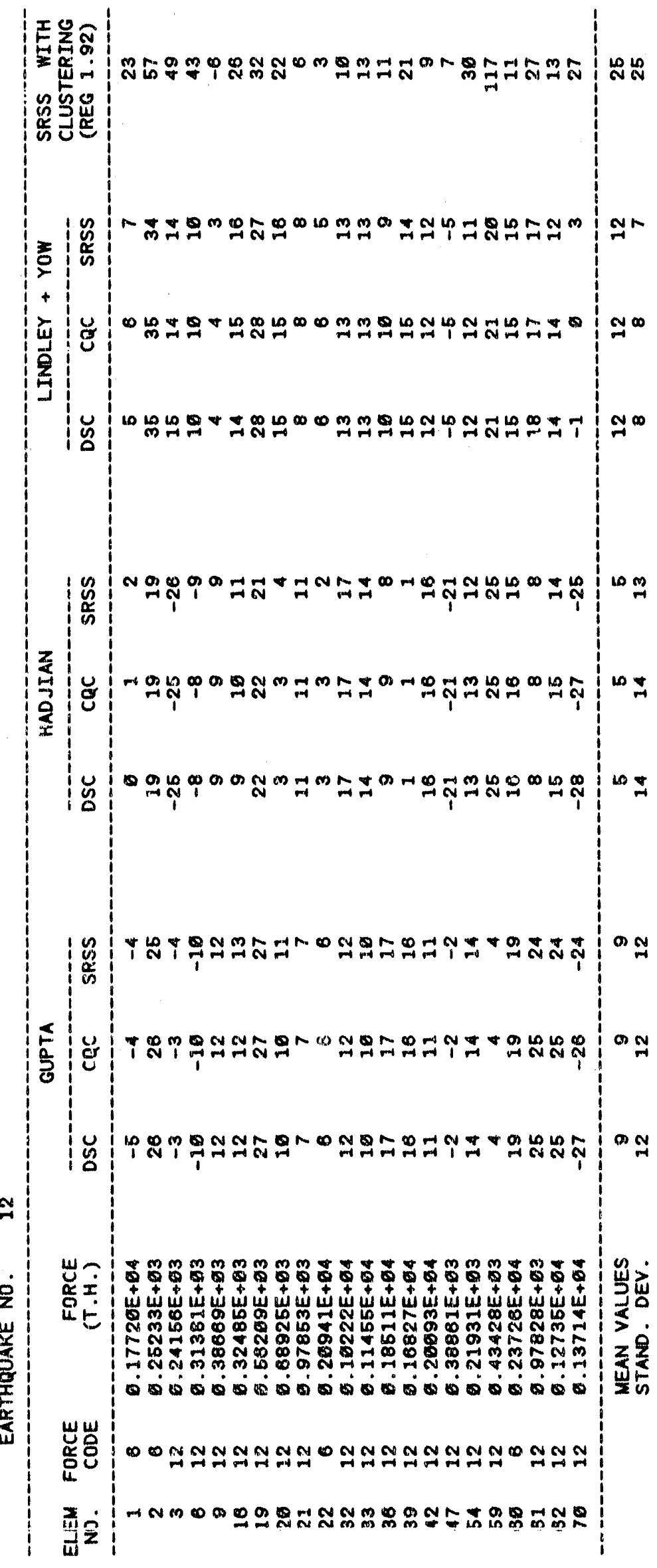

$A-25$ 


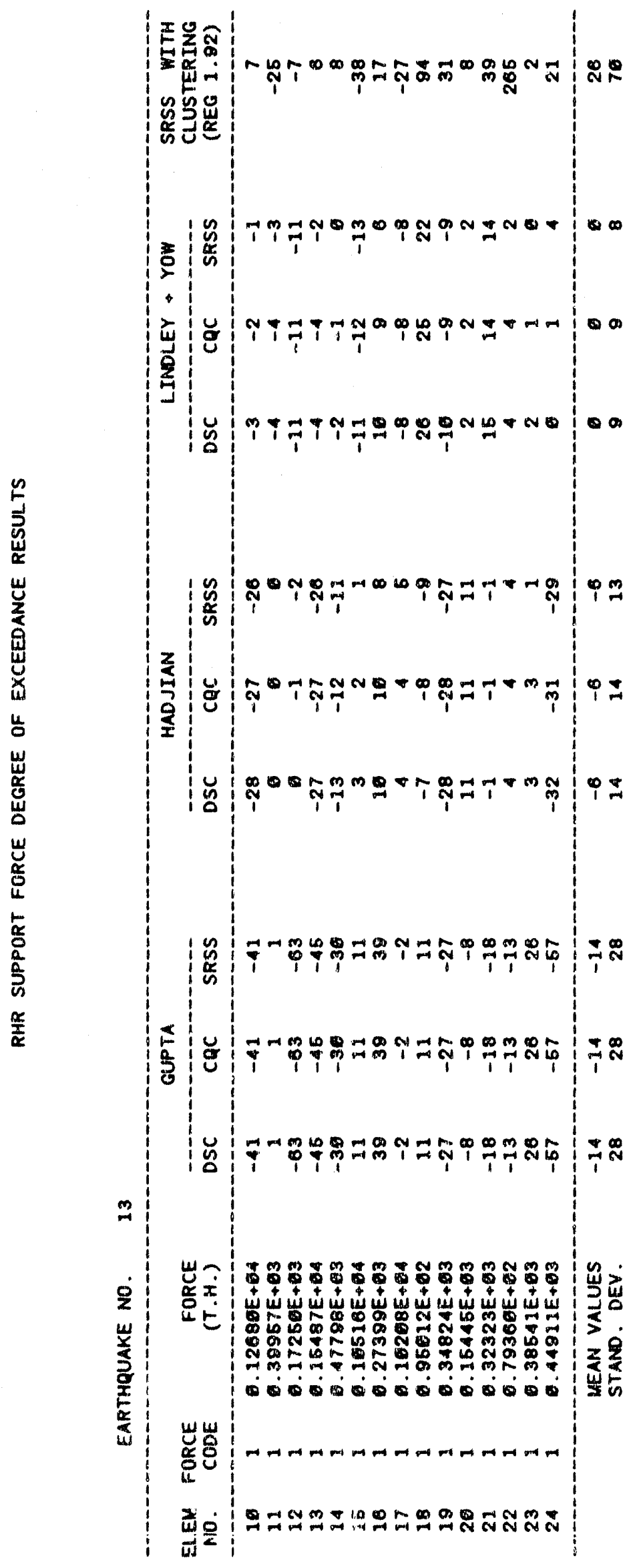

A -26 


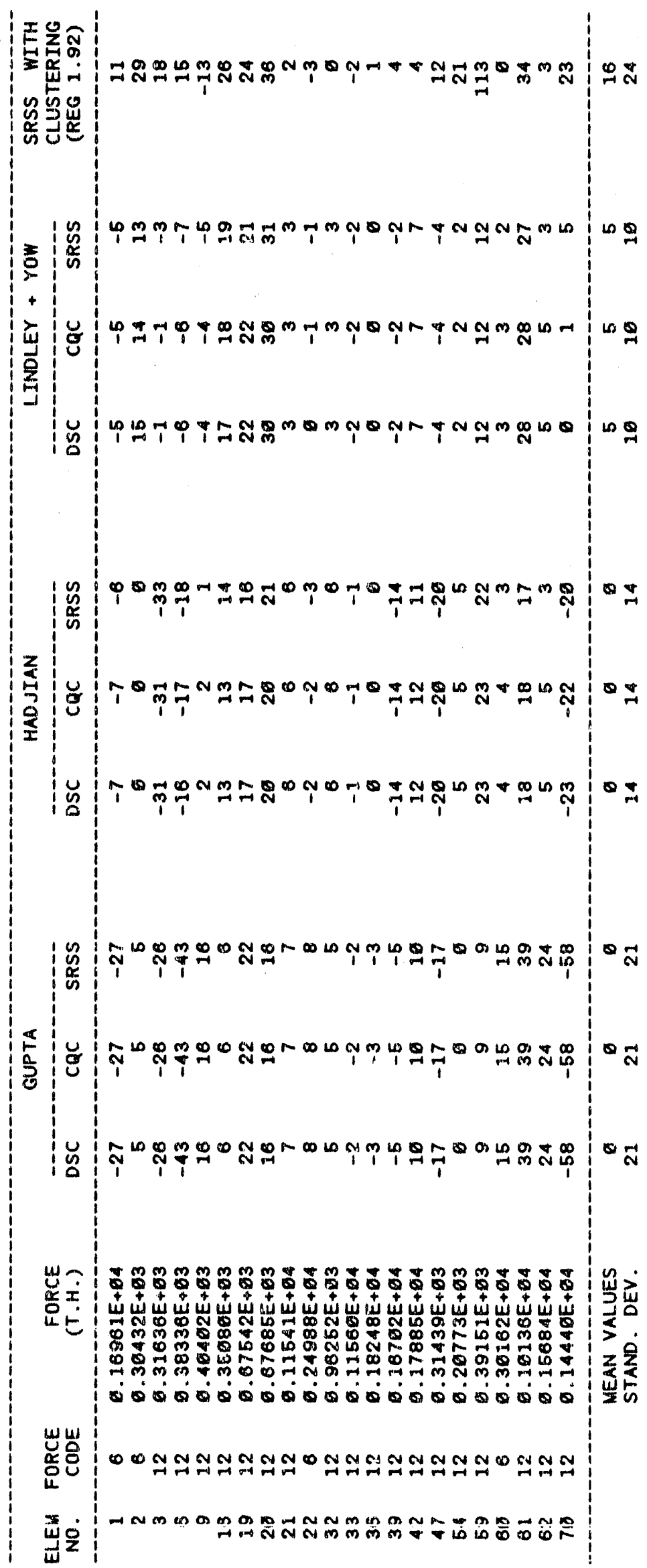




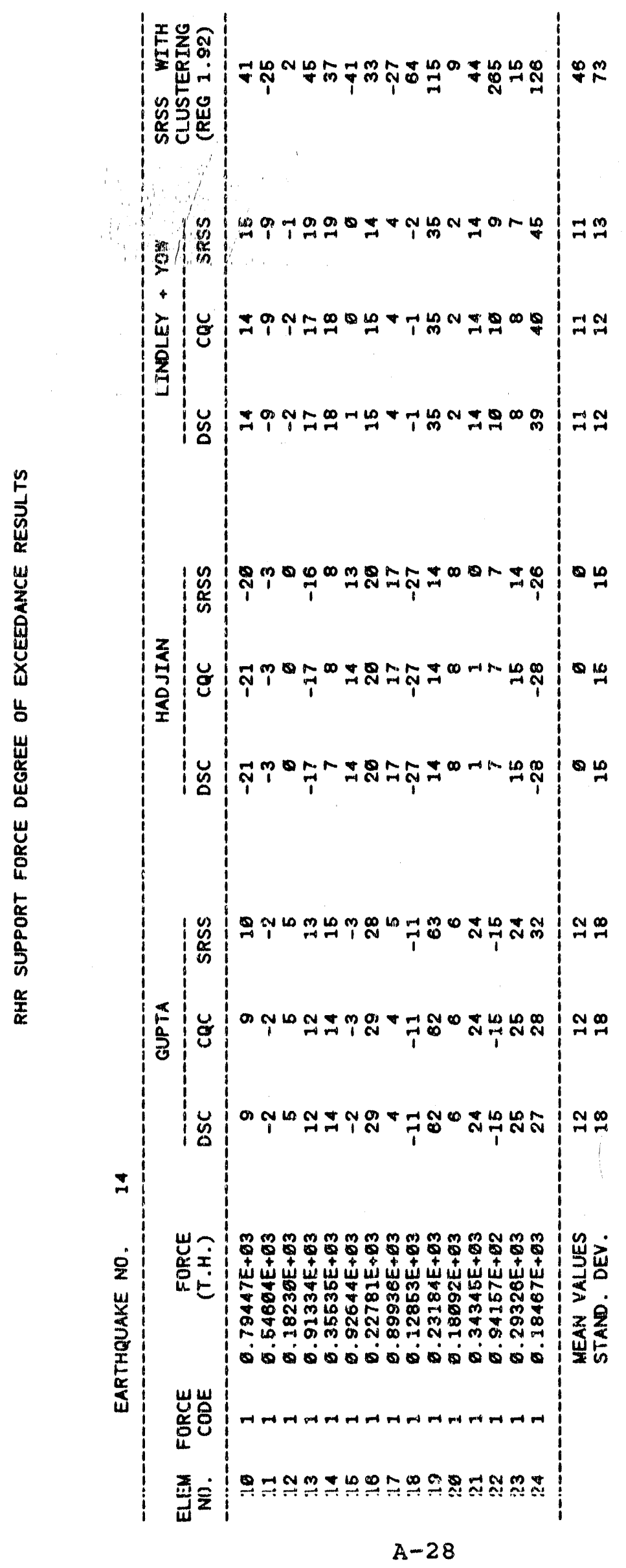




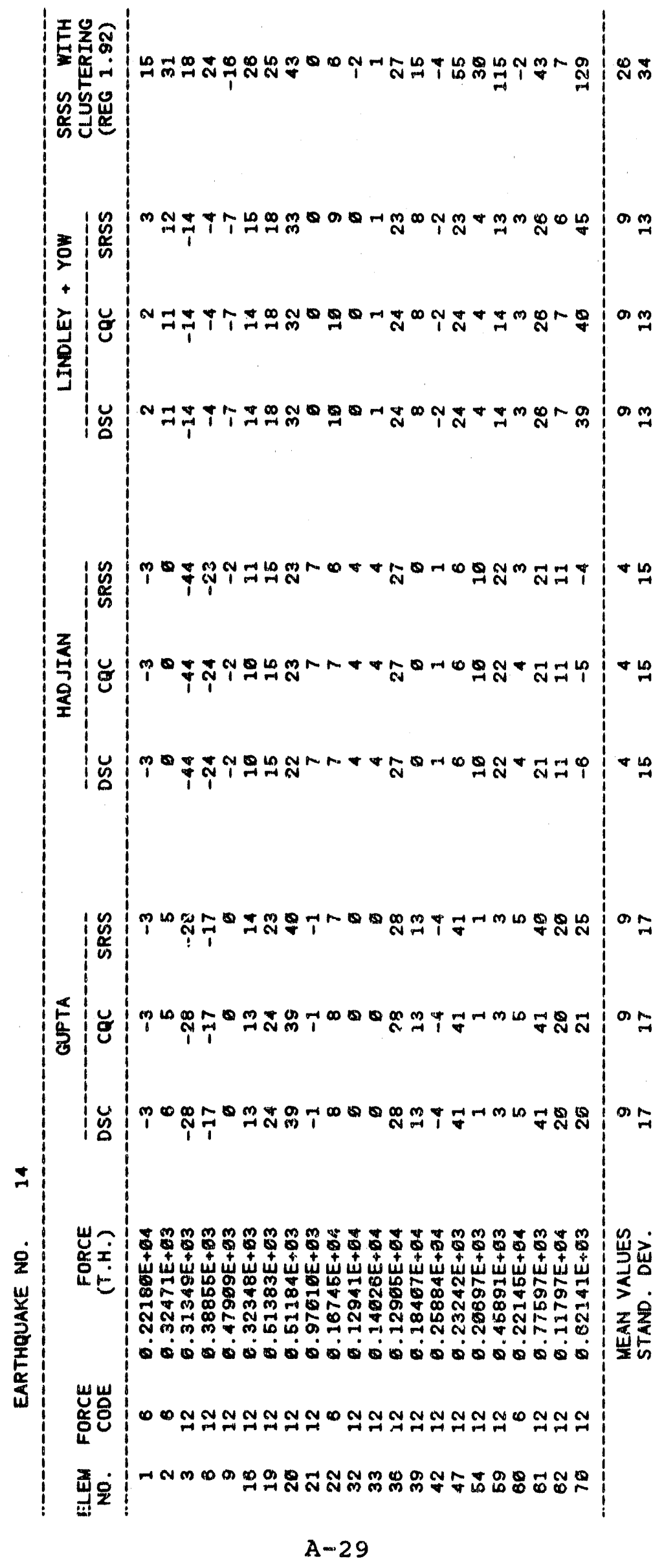




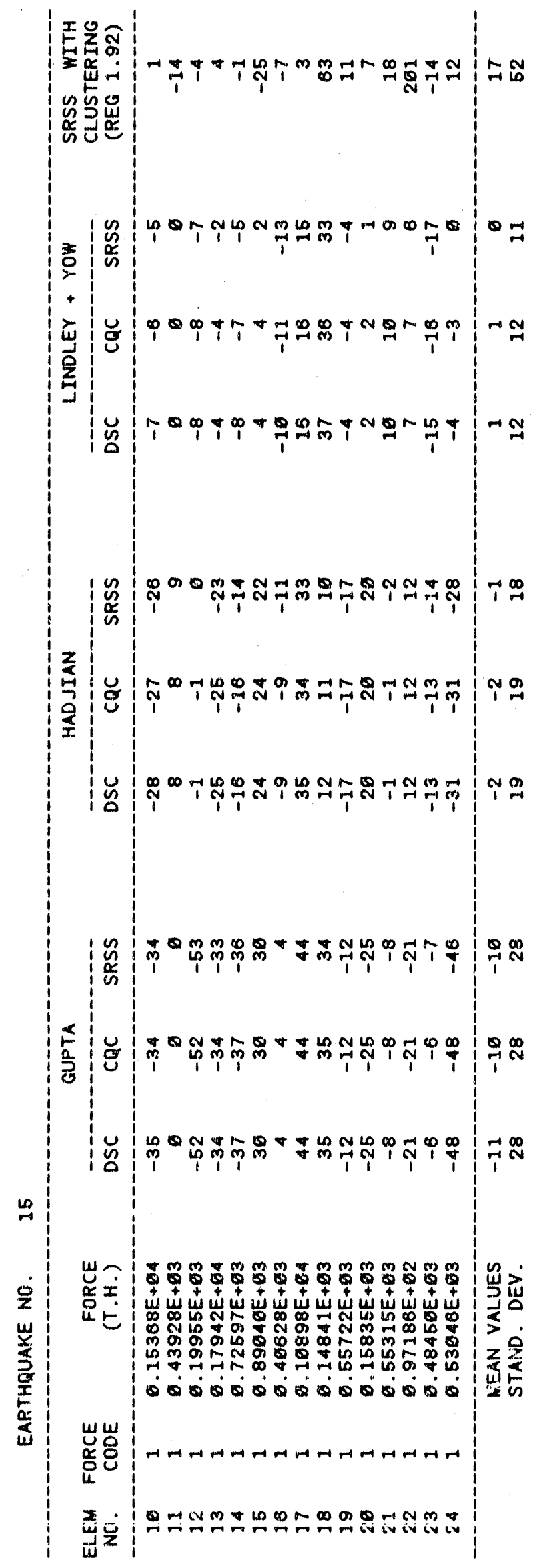

A -30 


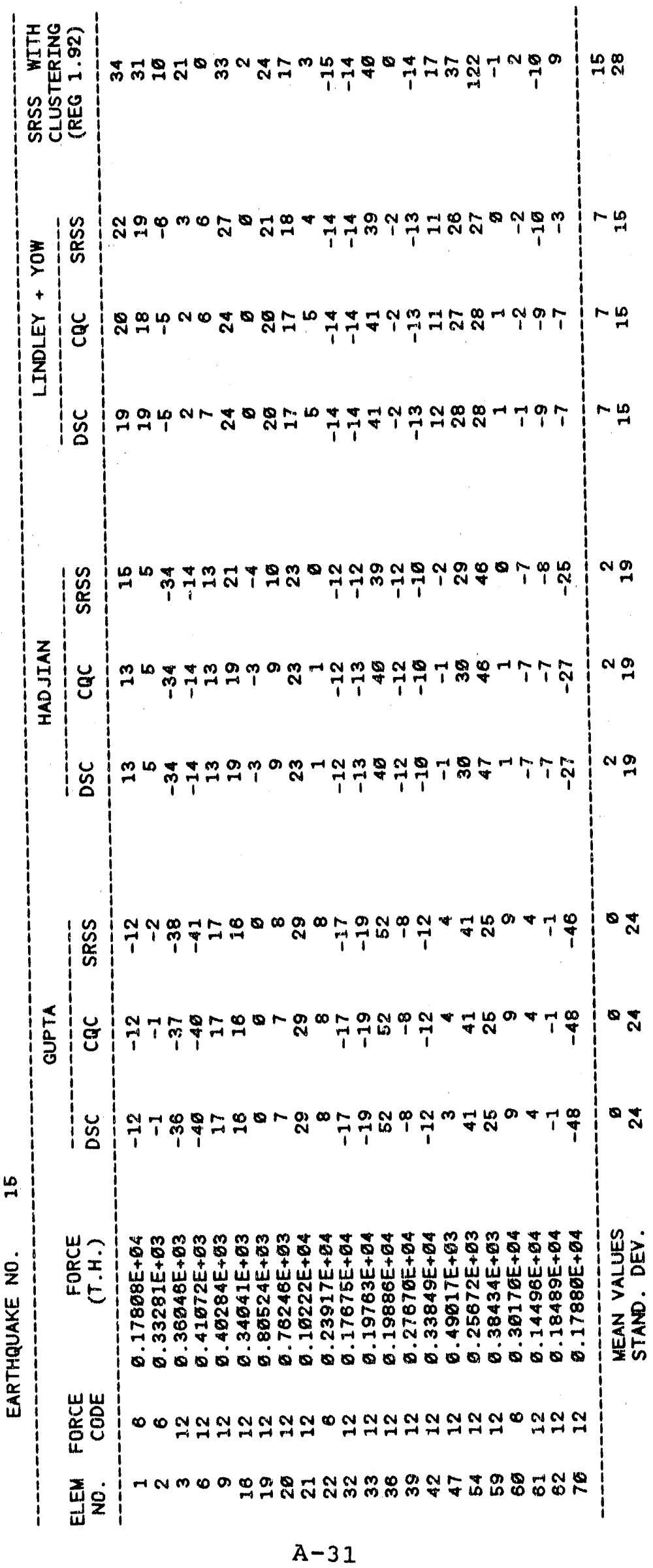




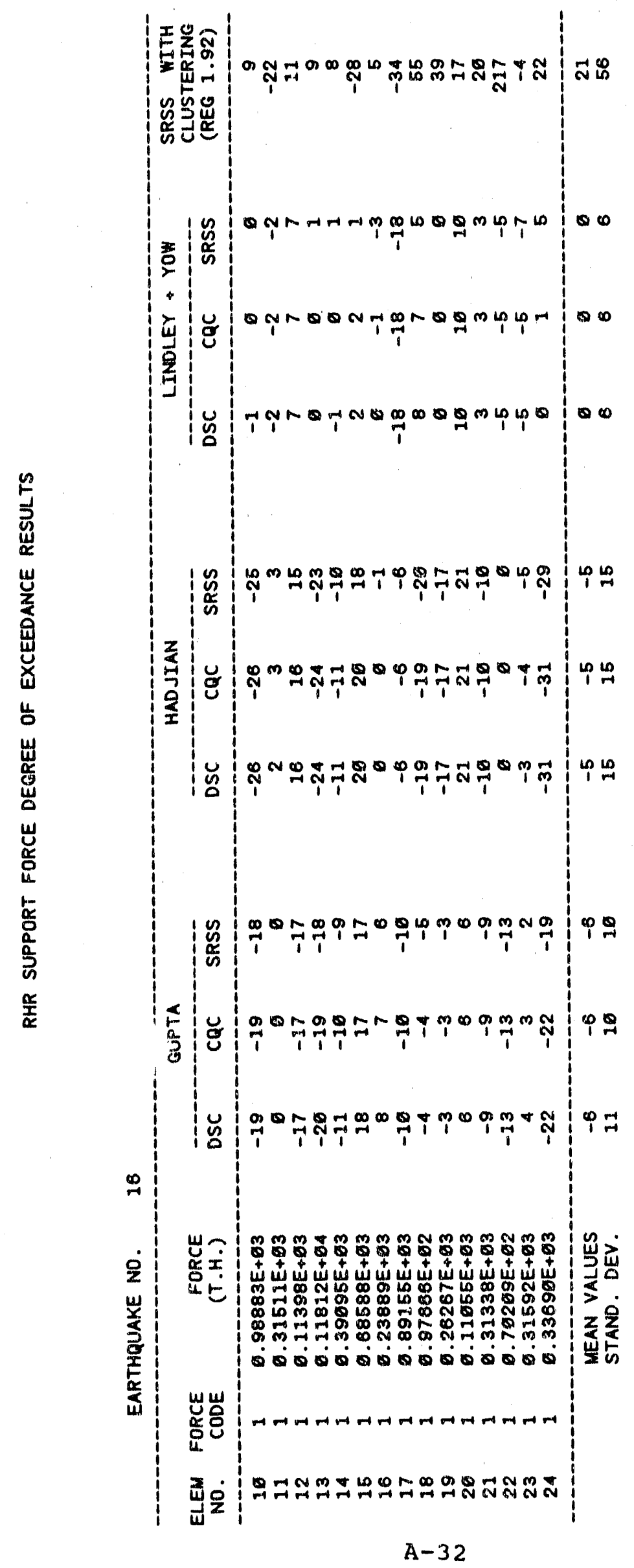




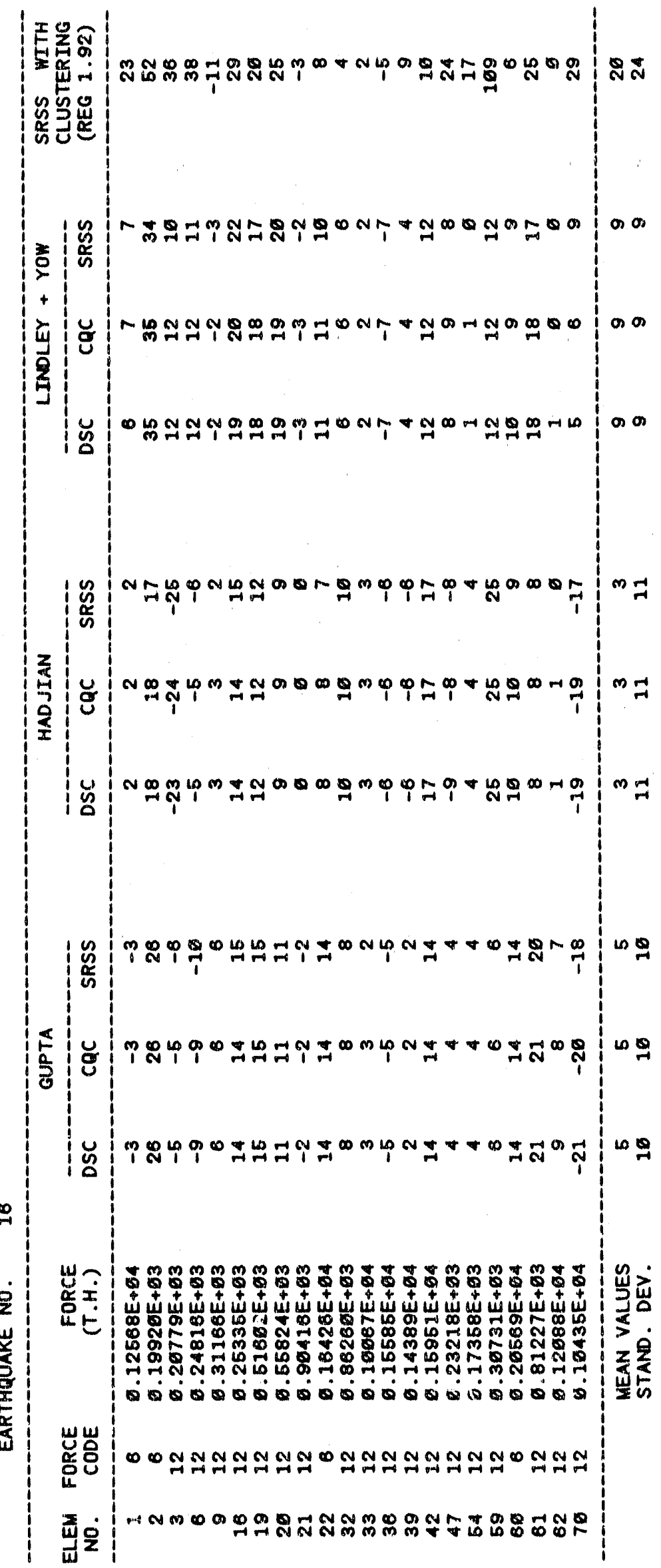




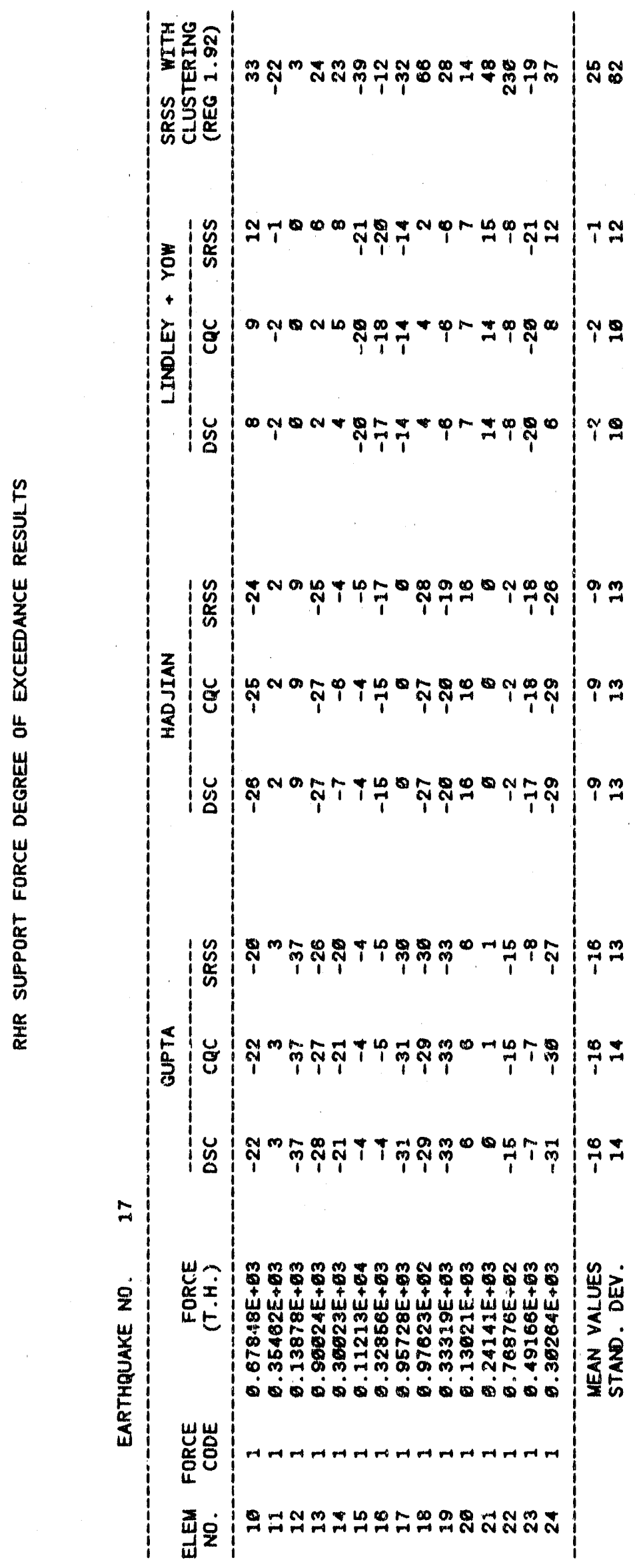

A -34 


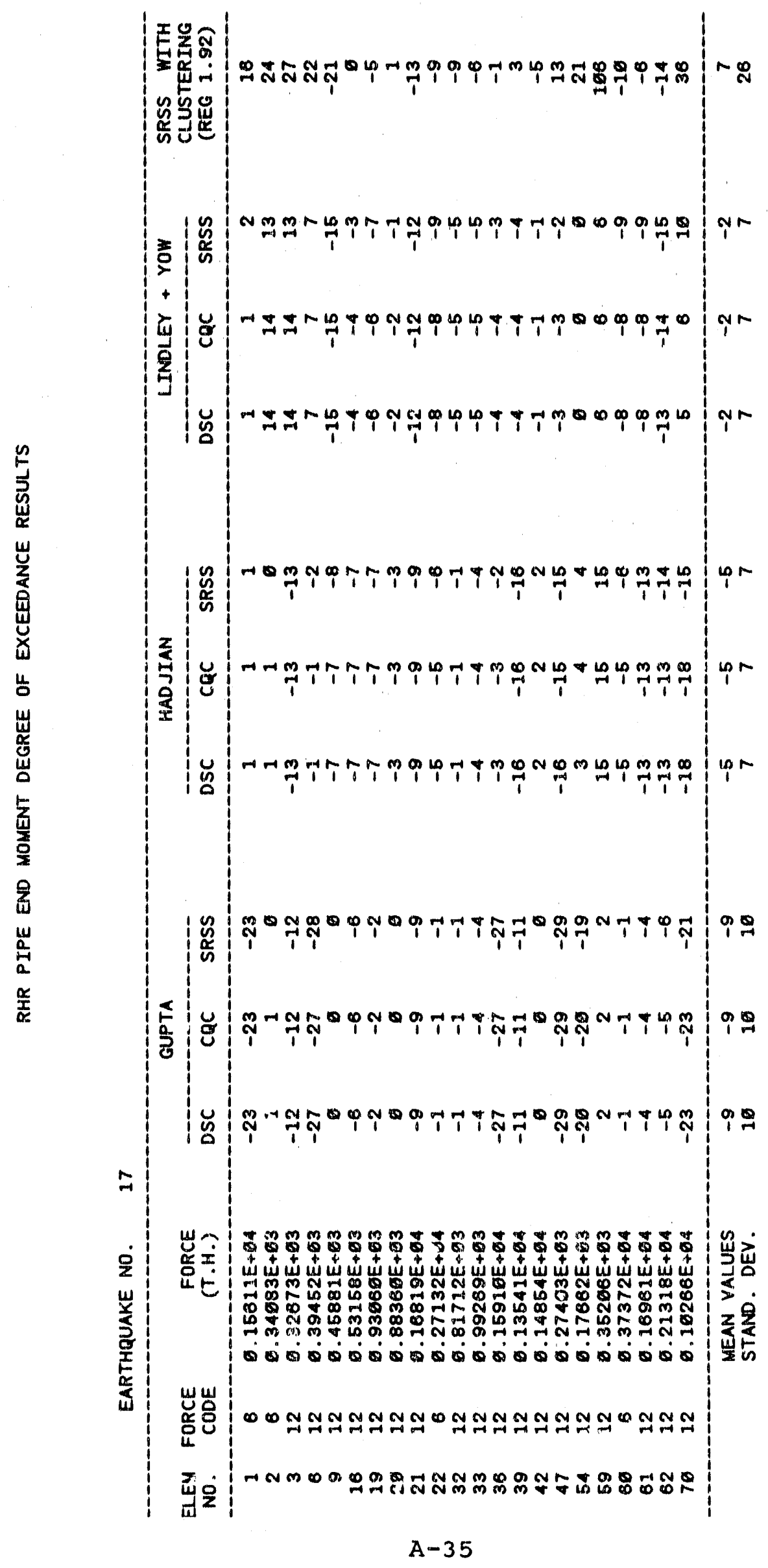




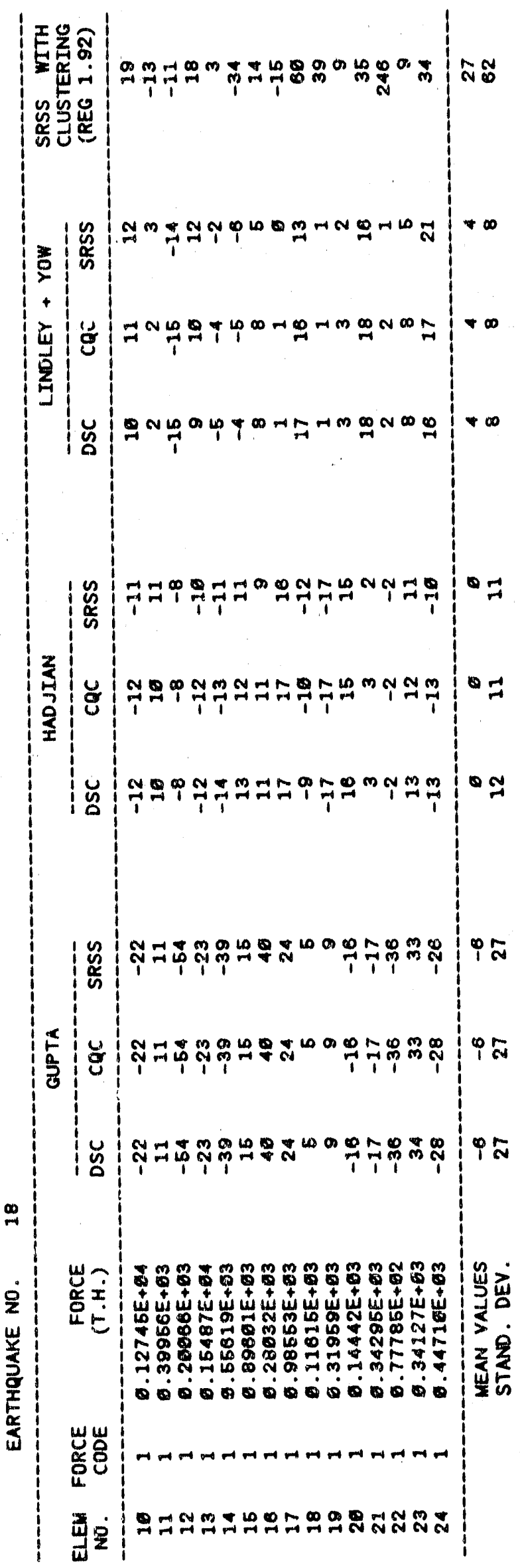

$A-36$ 


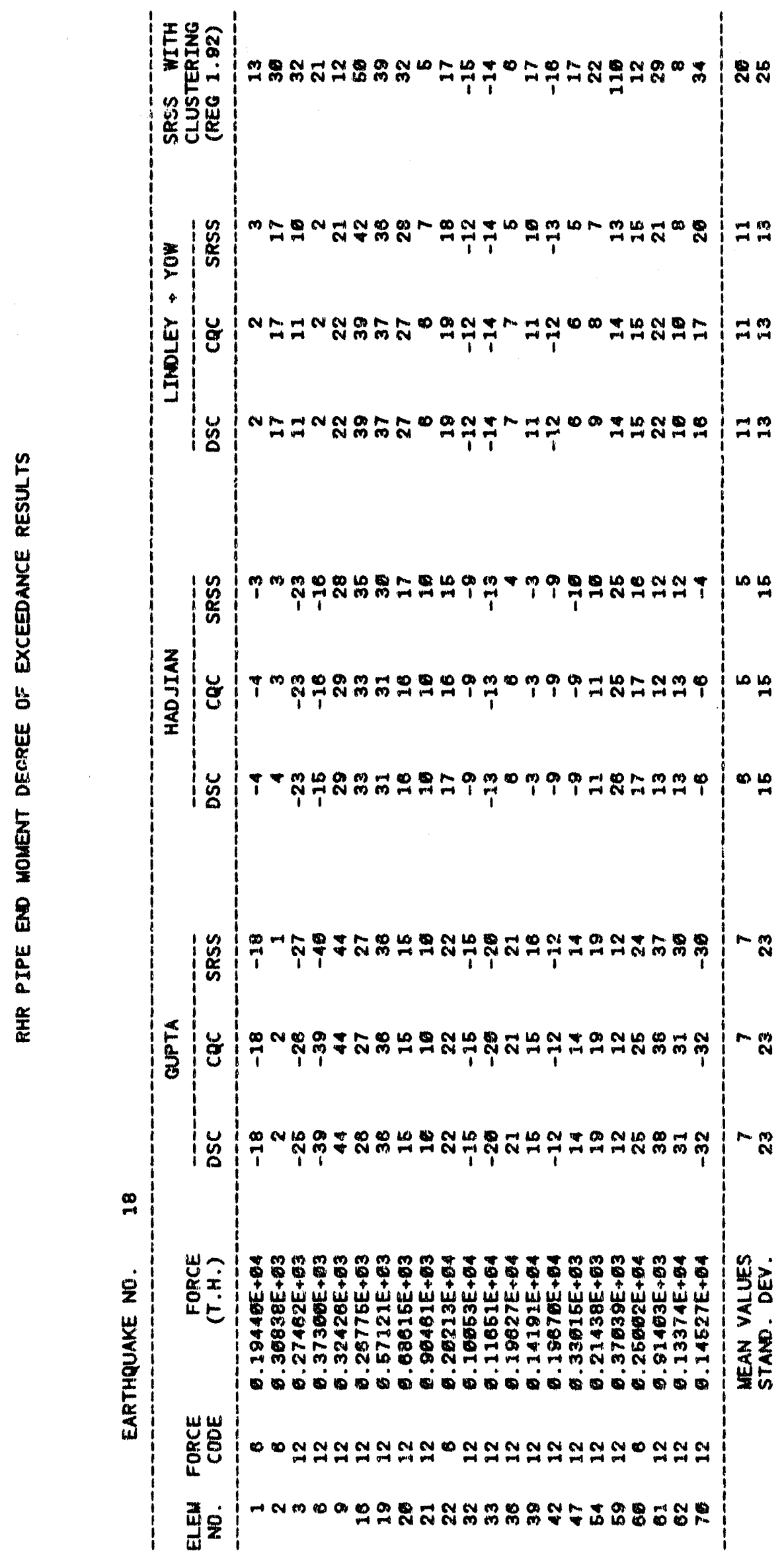




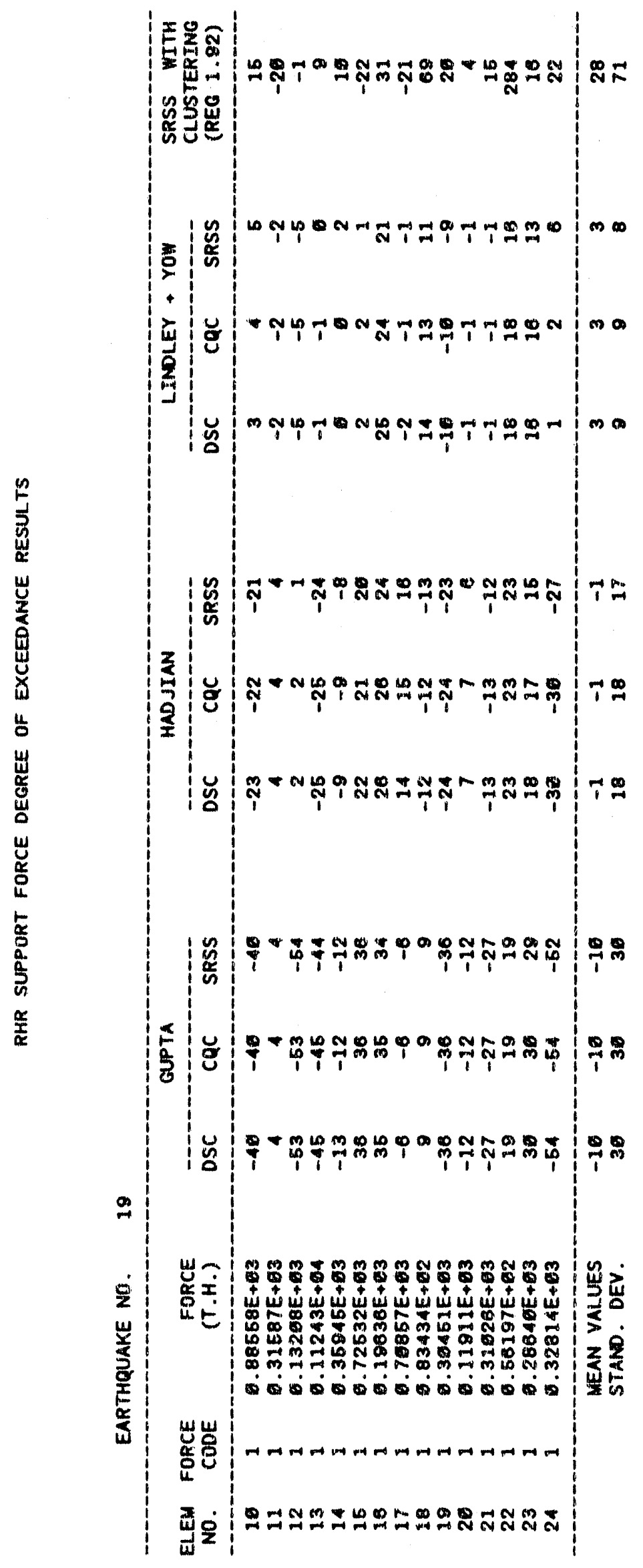

$A-38$ 


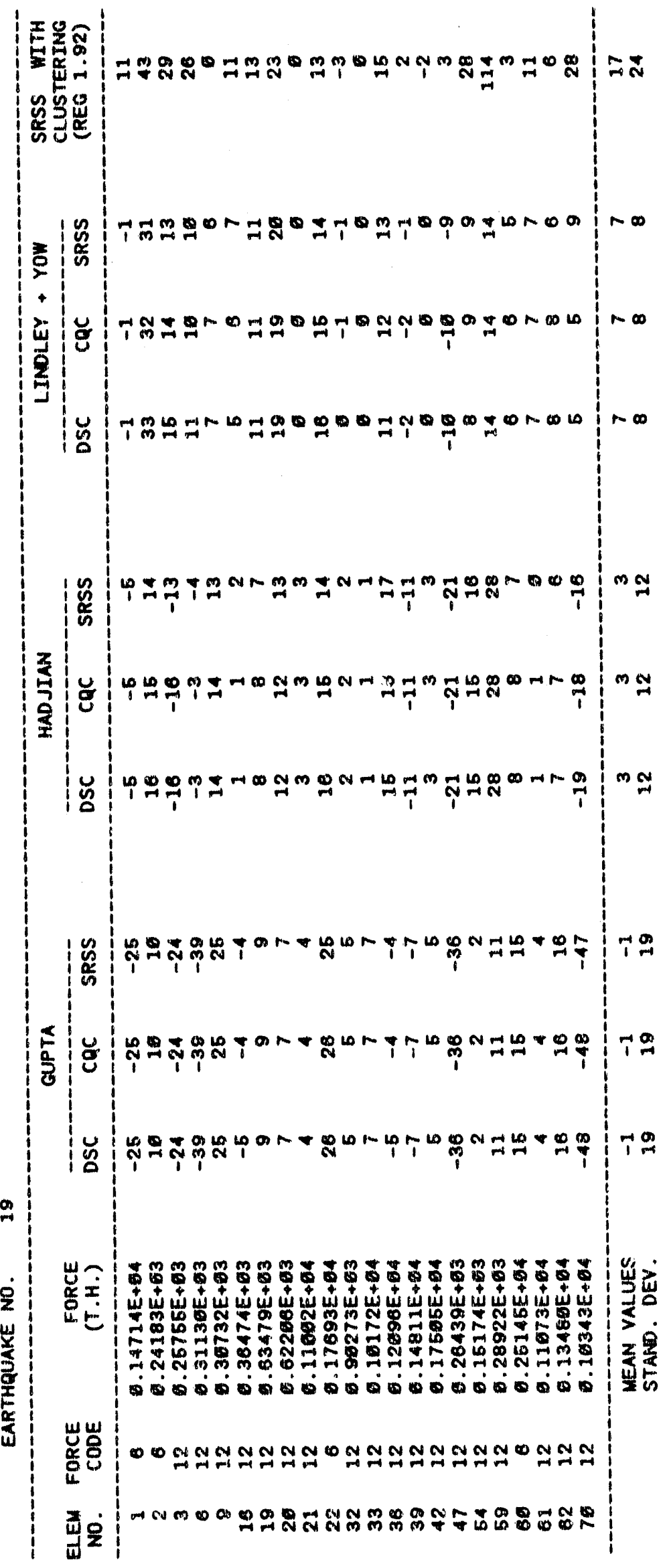

$$
A-39
$$




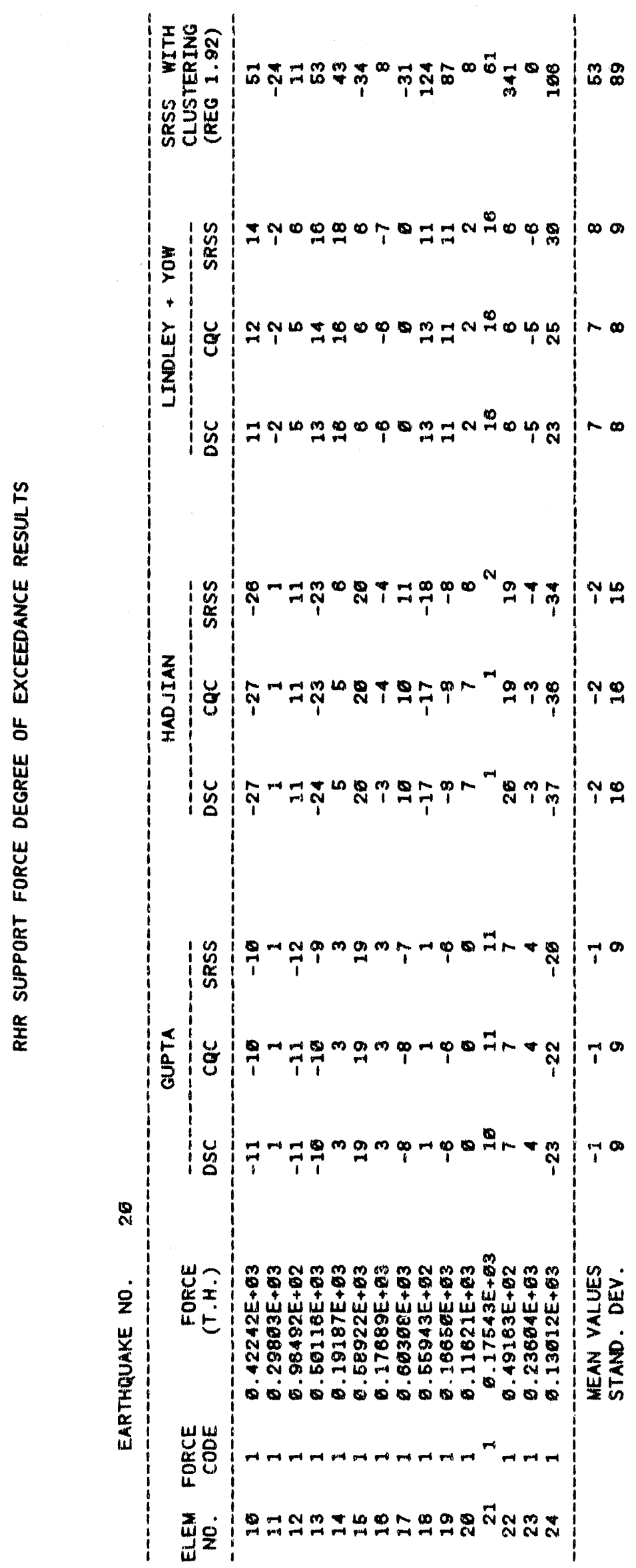

$A-40$ 


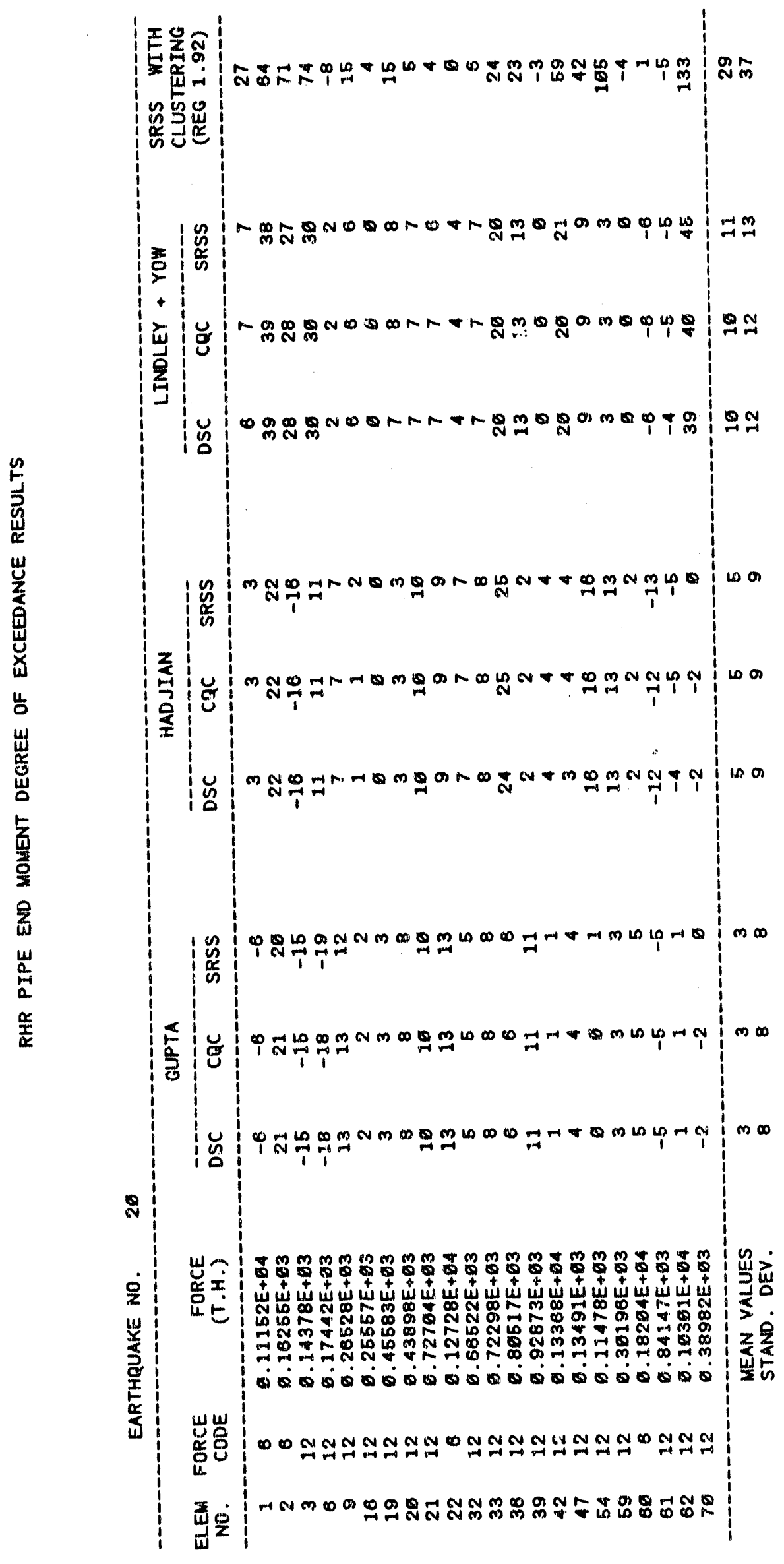

$A-41$ 


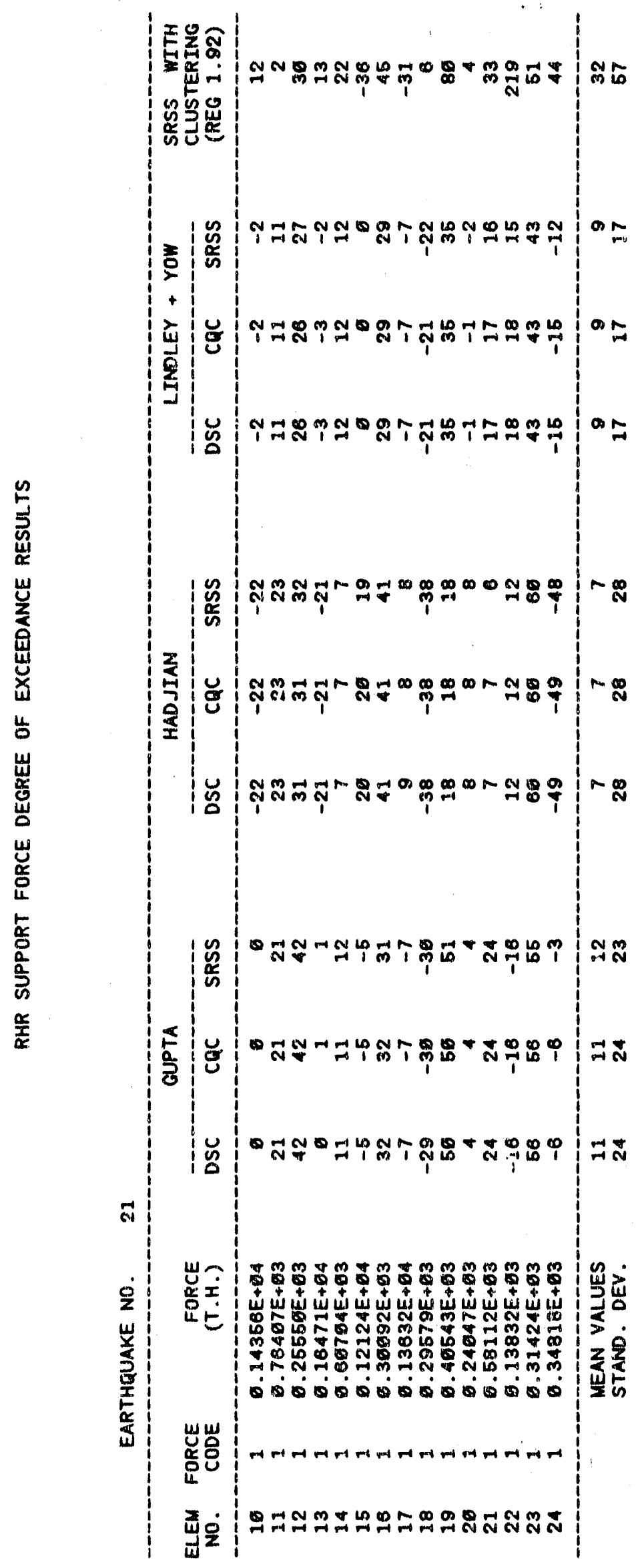

$$
A-42
$$




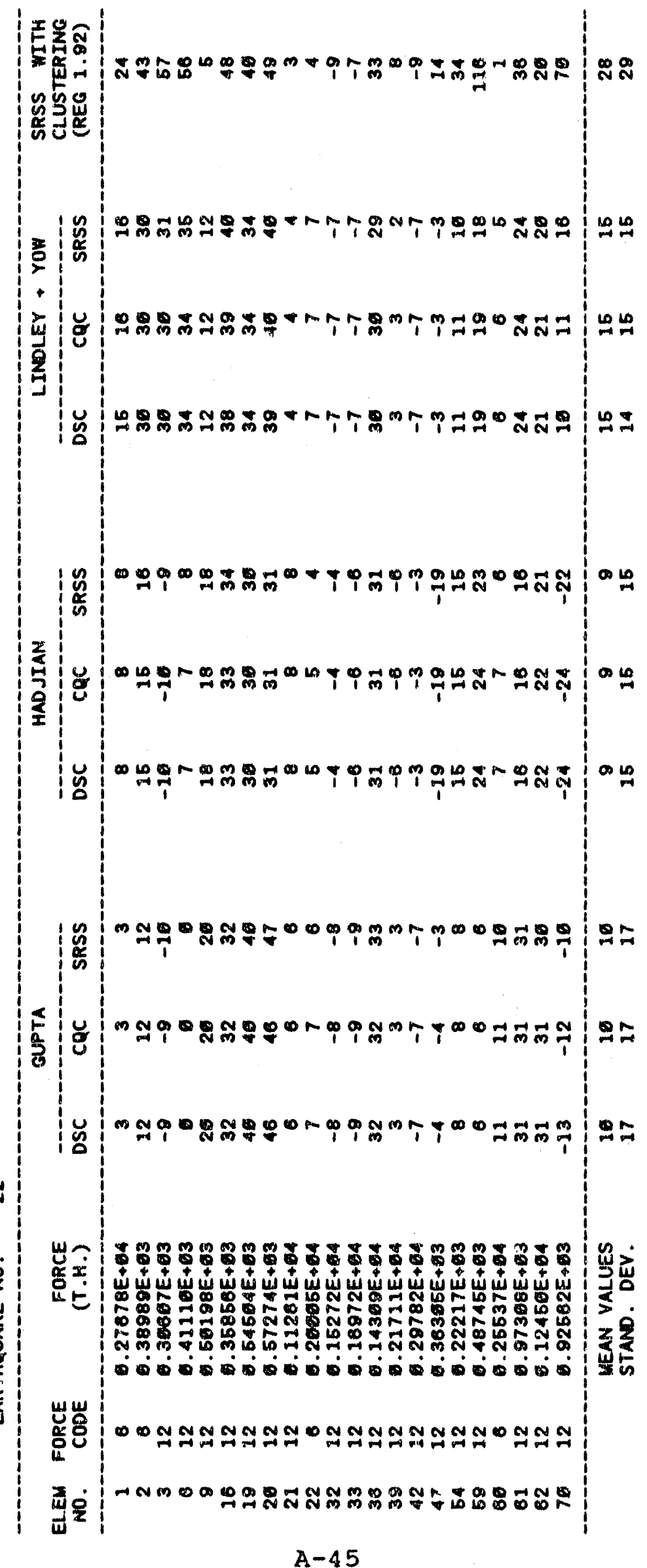




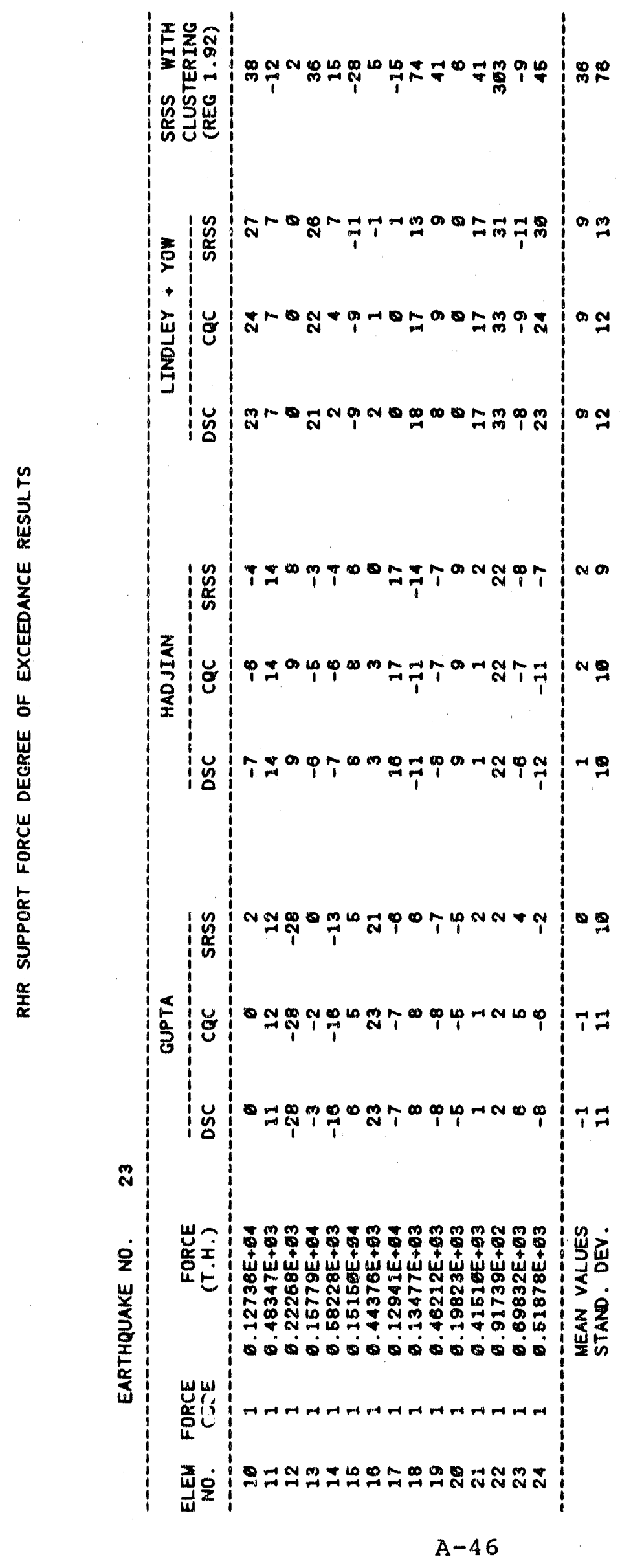




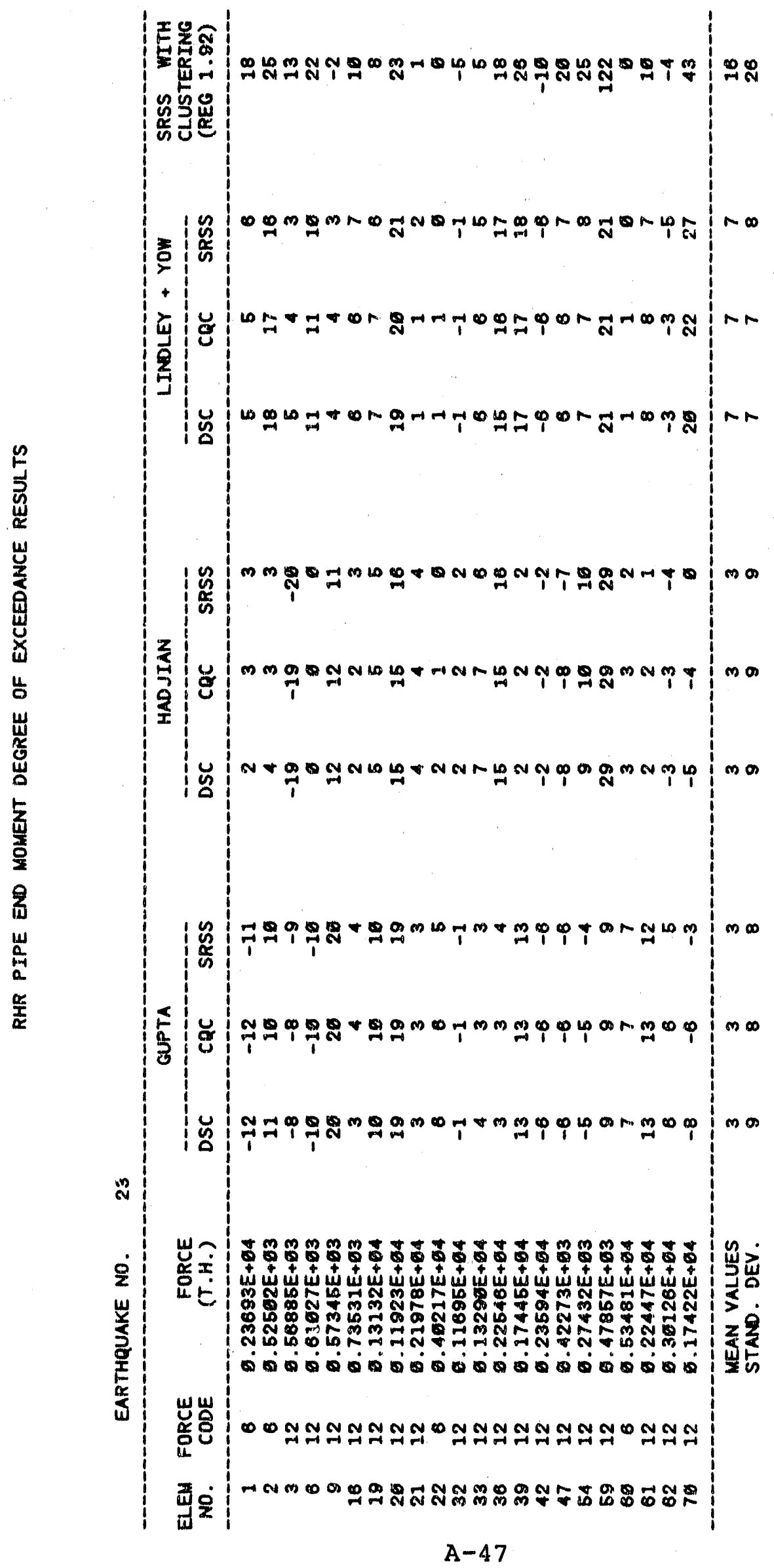




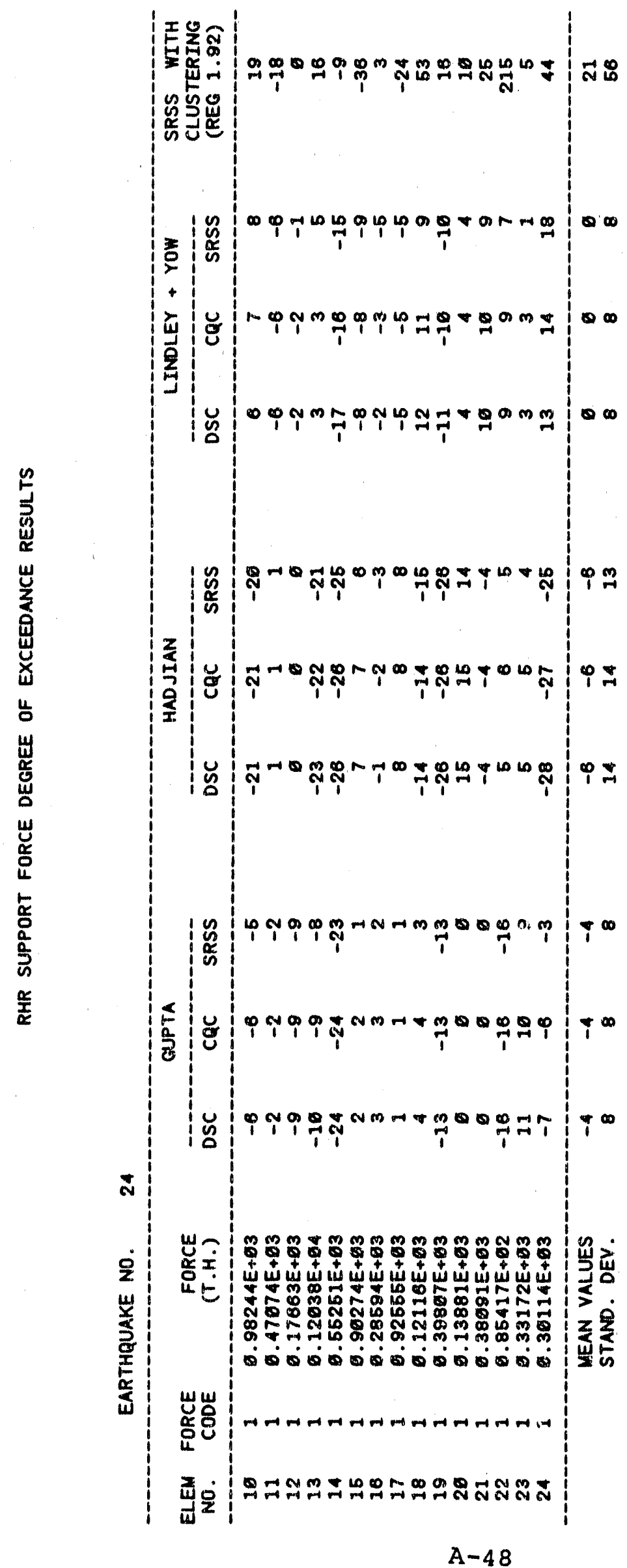




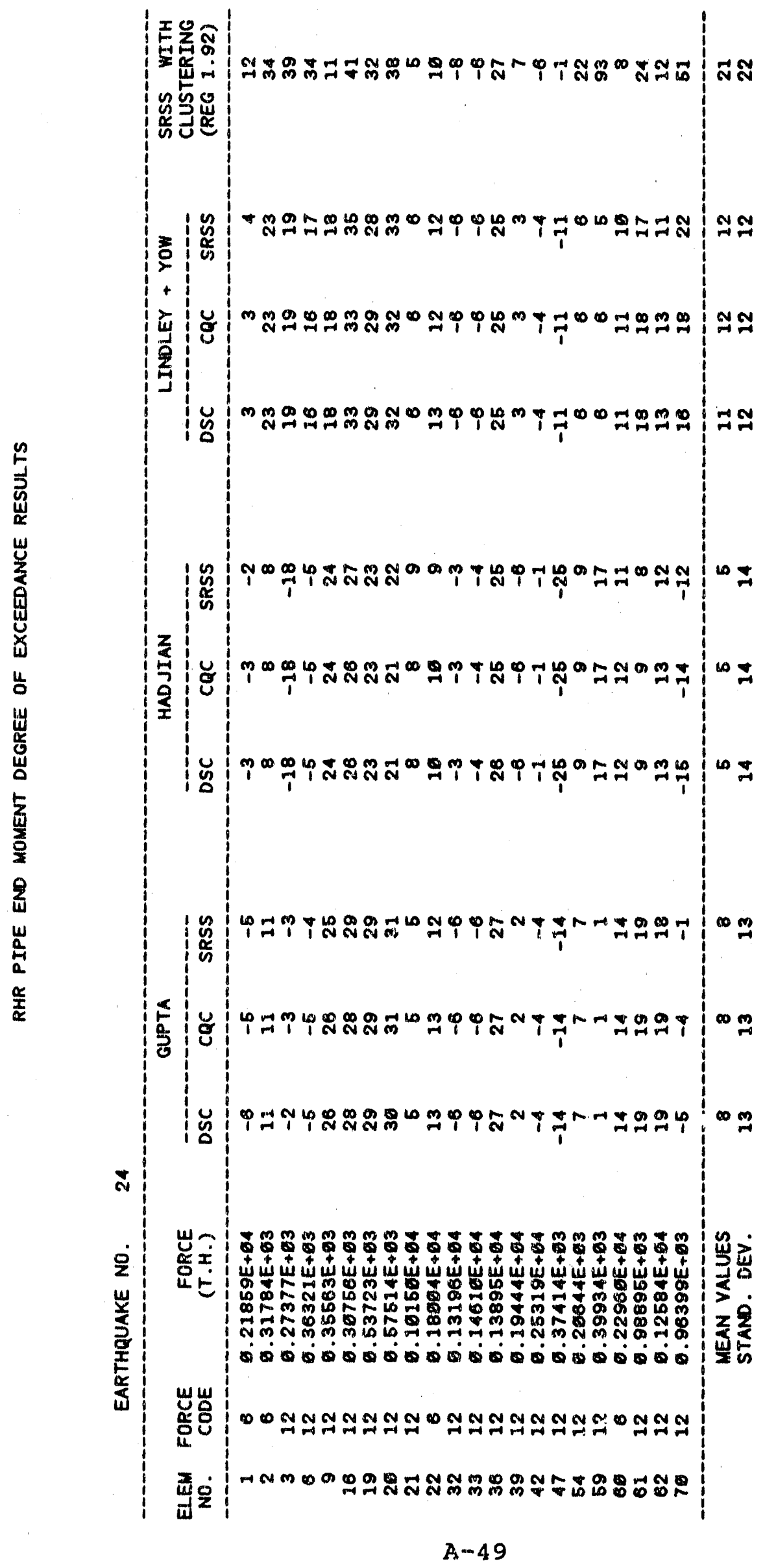




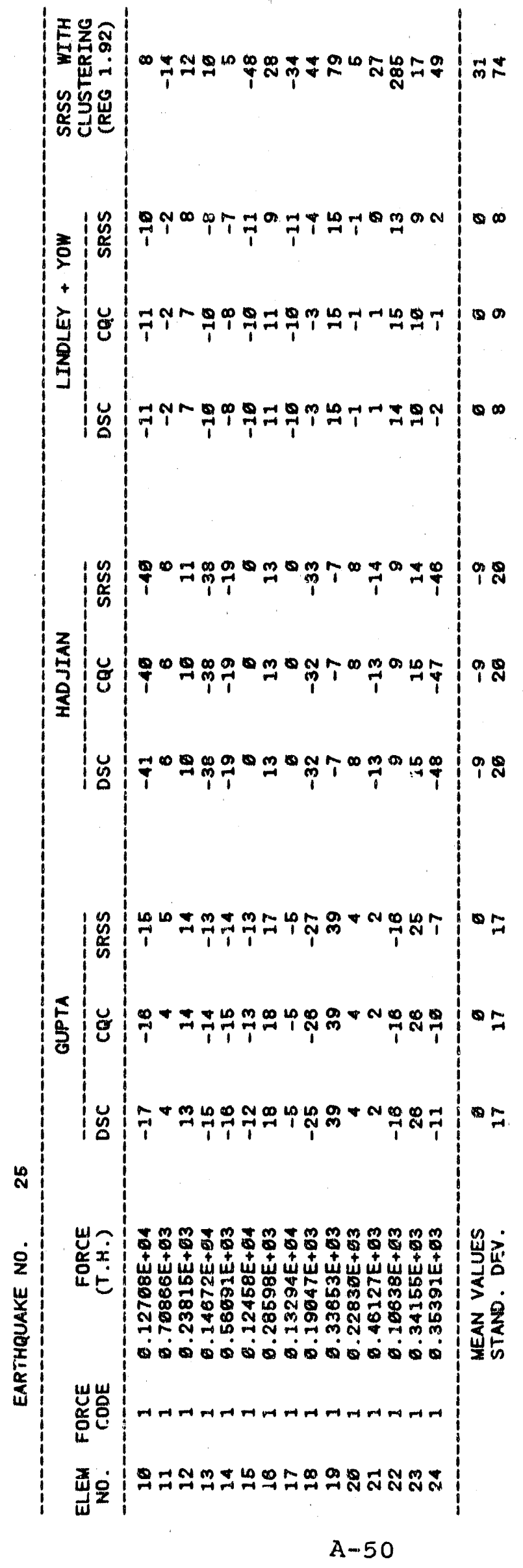




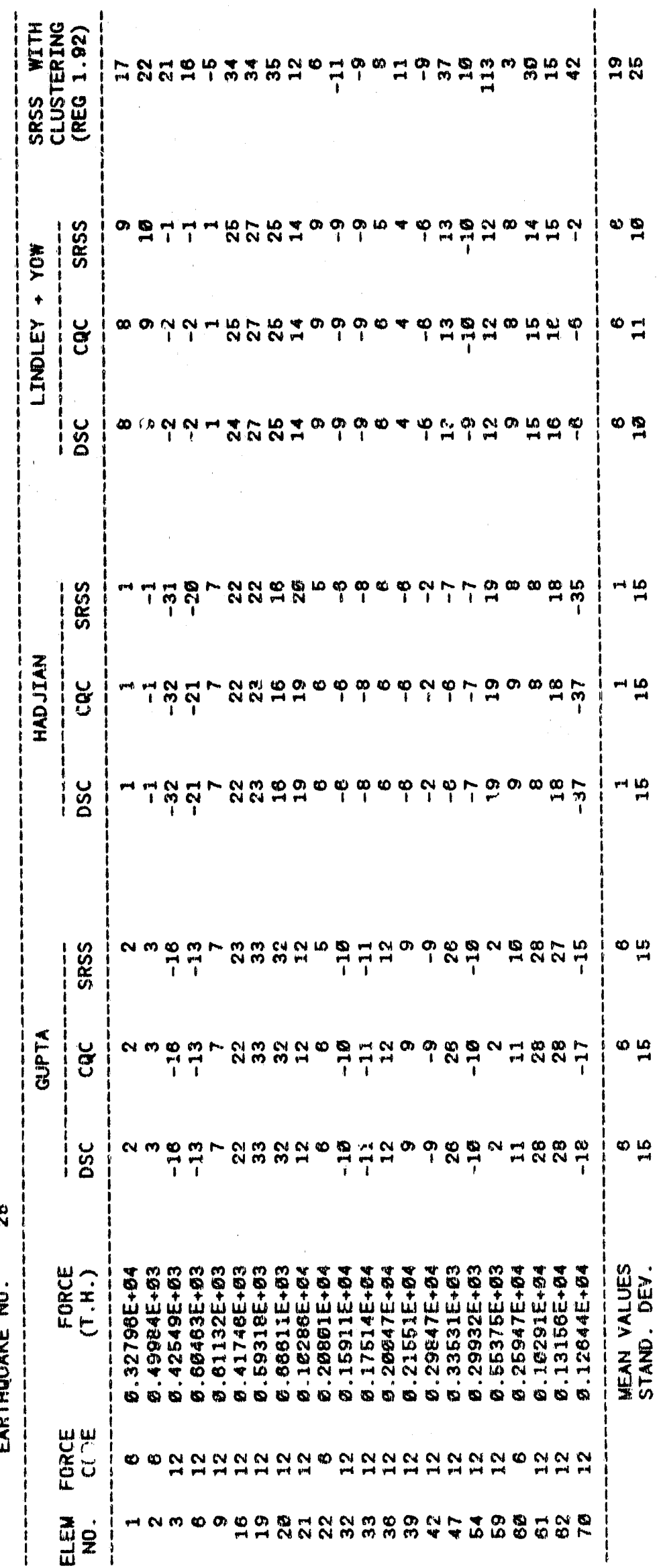

$A-51$ 


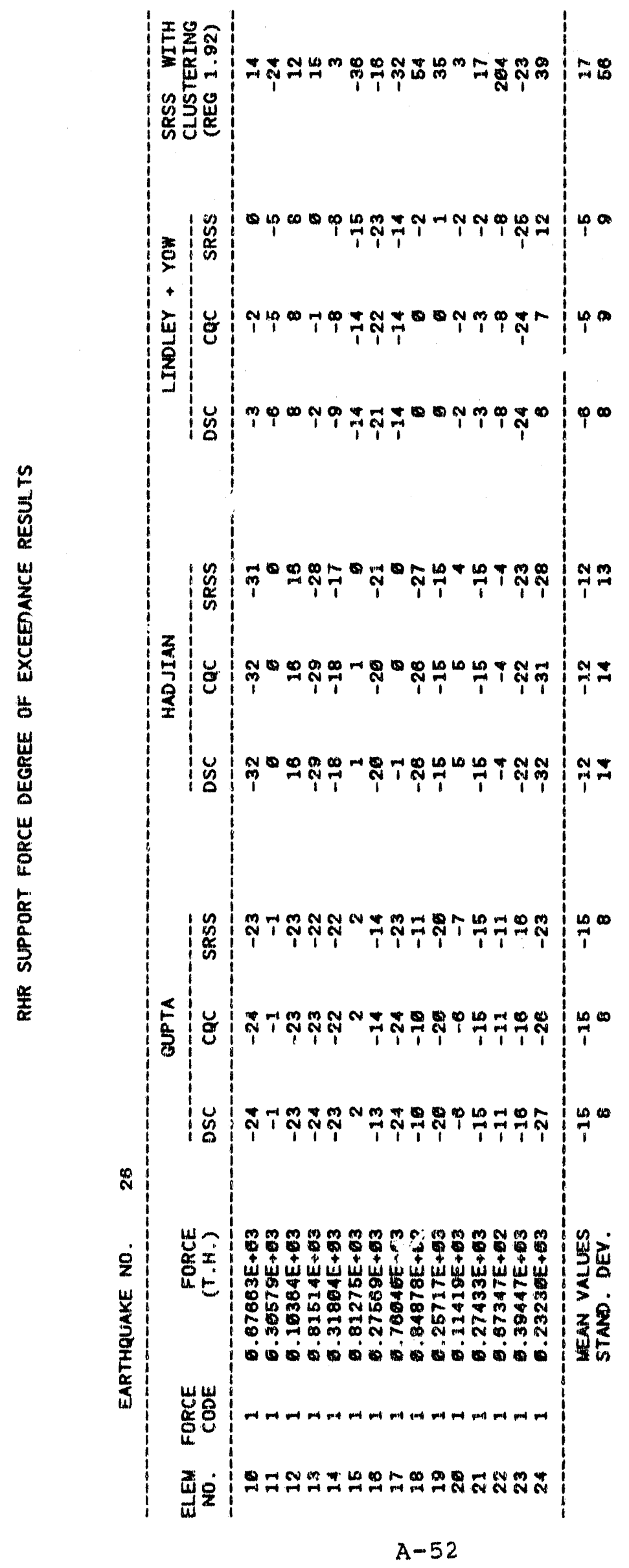




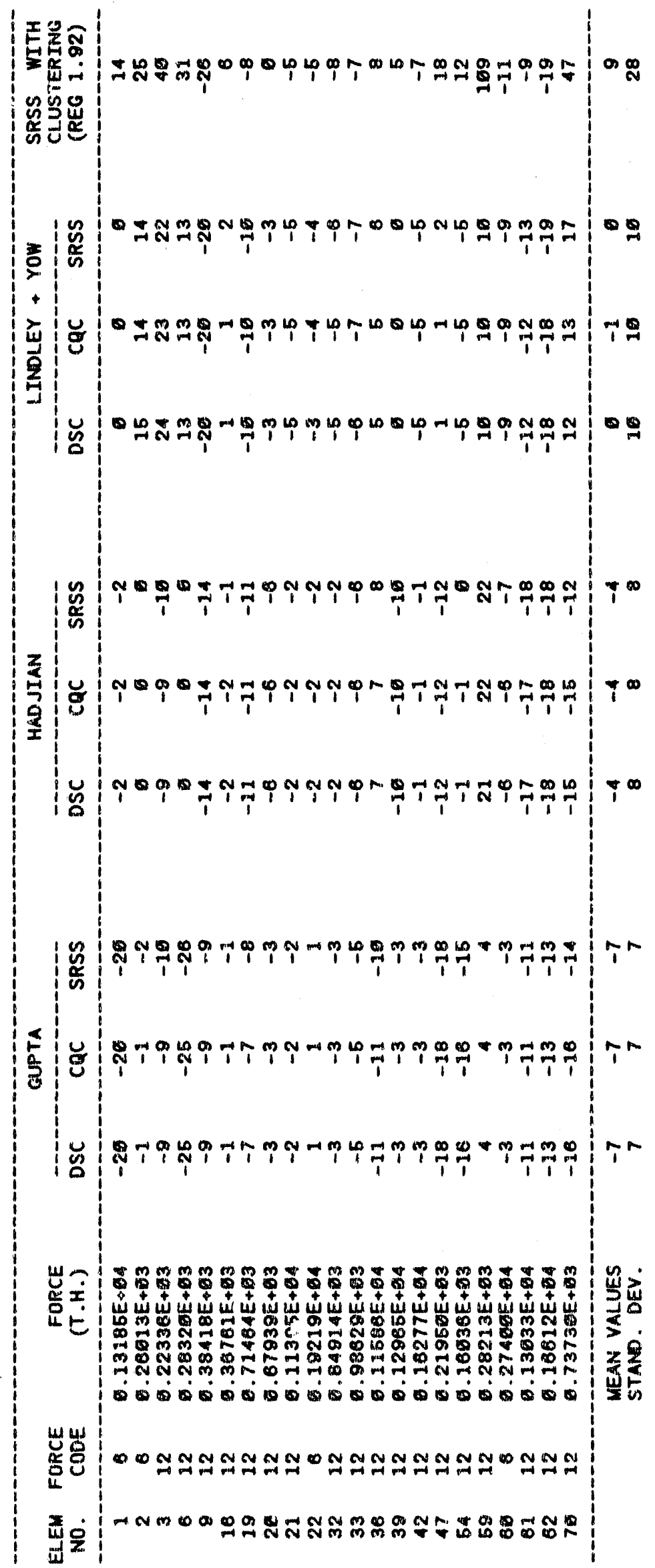

$$
\text { A }-53
$$




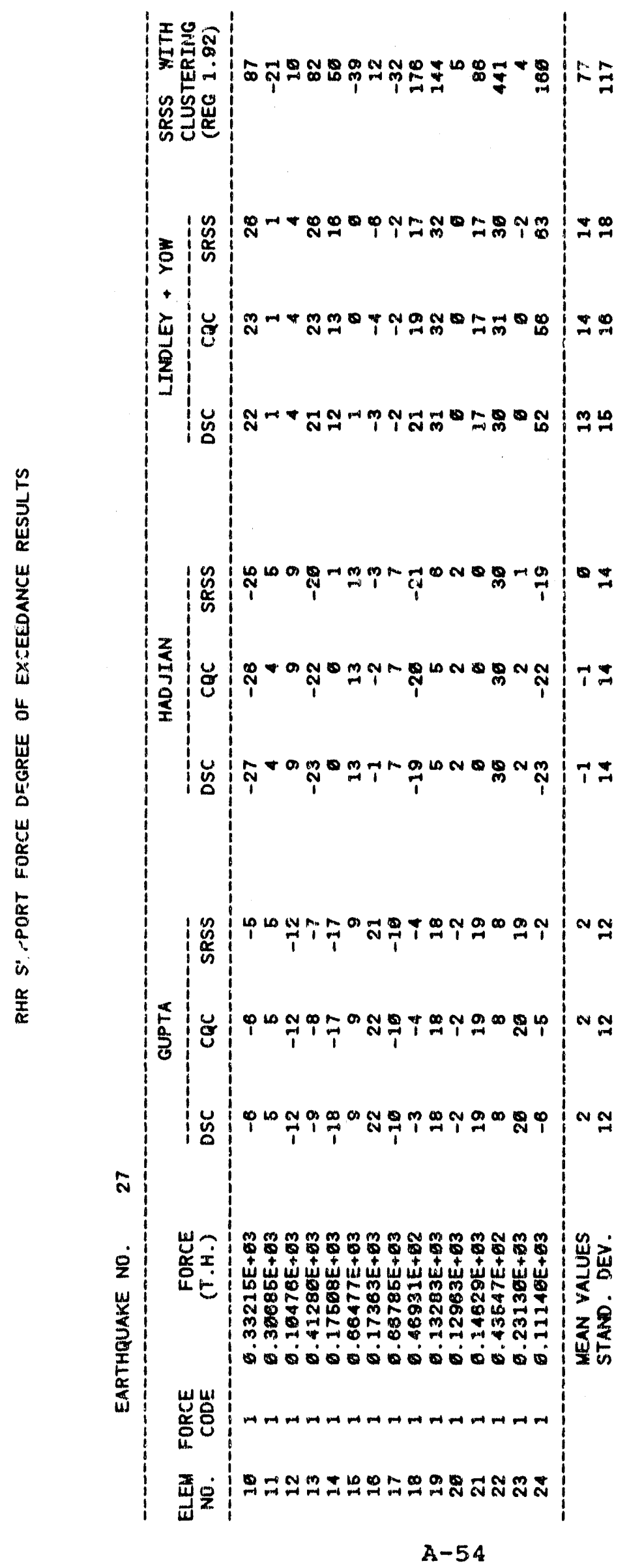




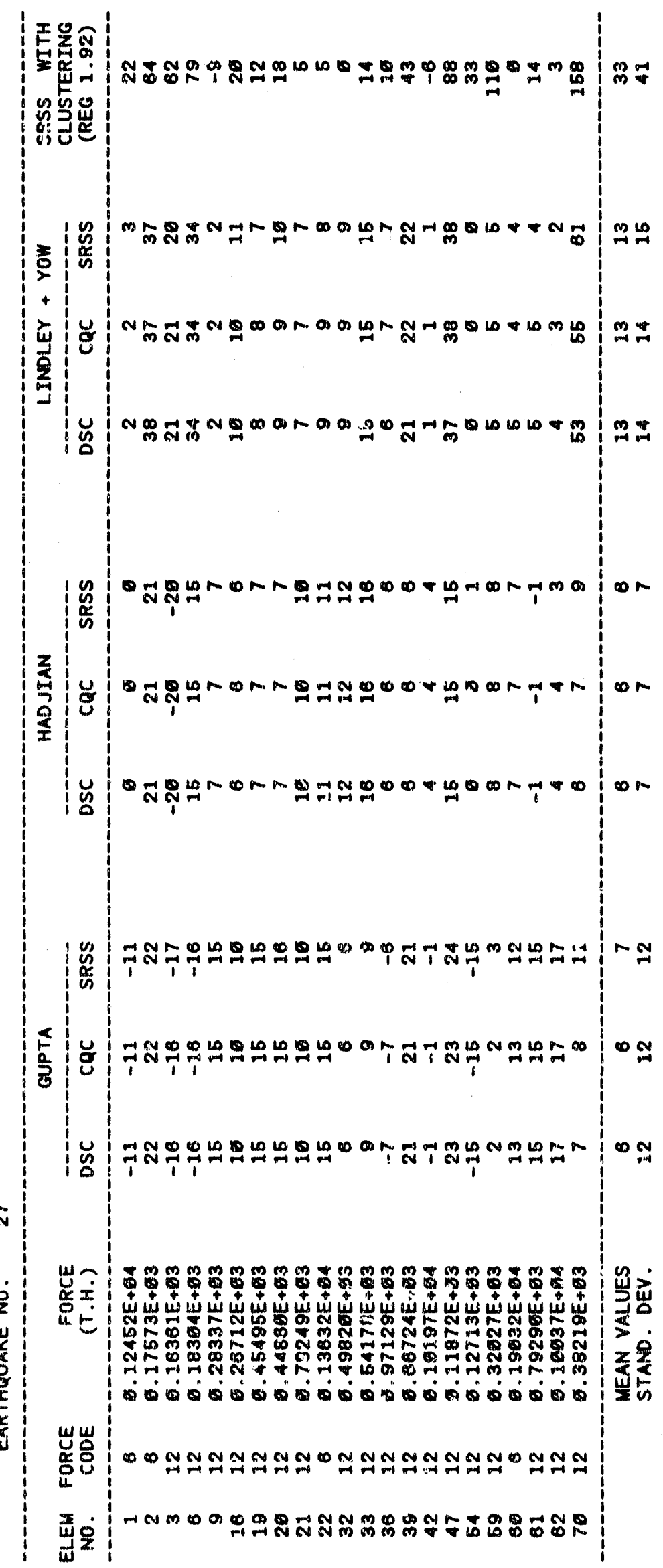




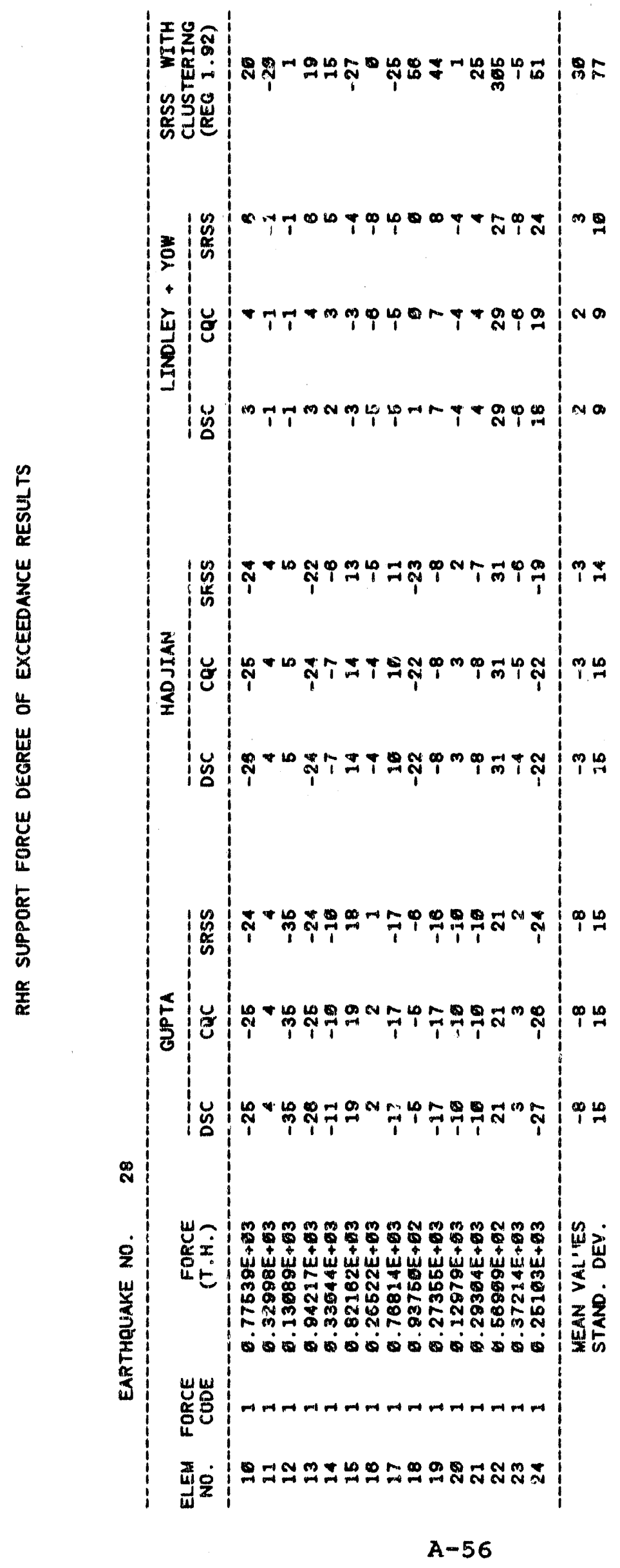




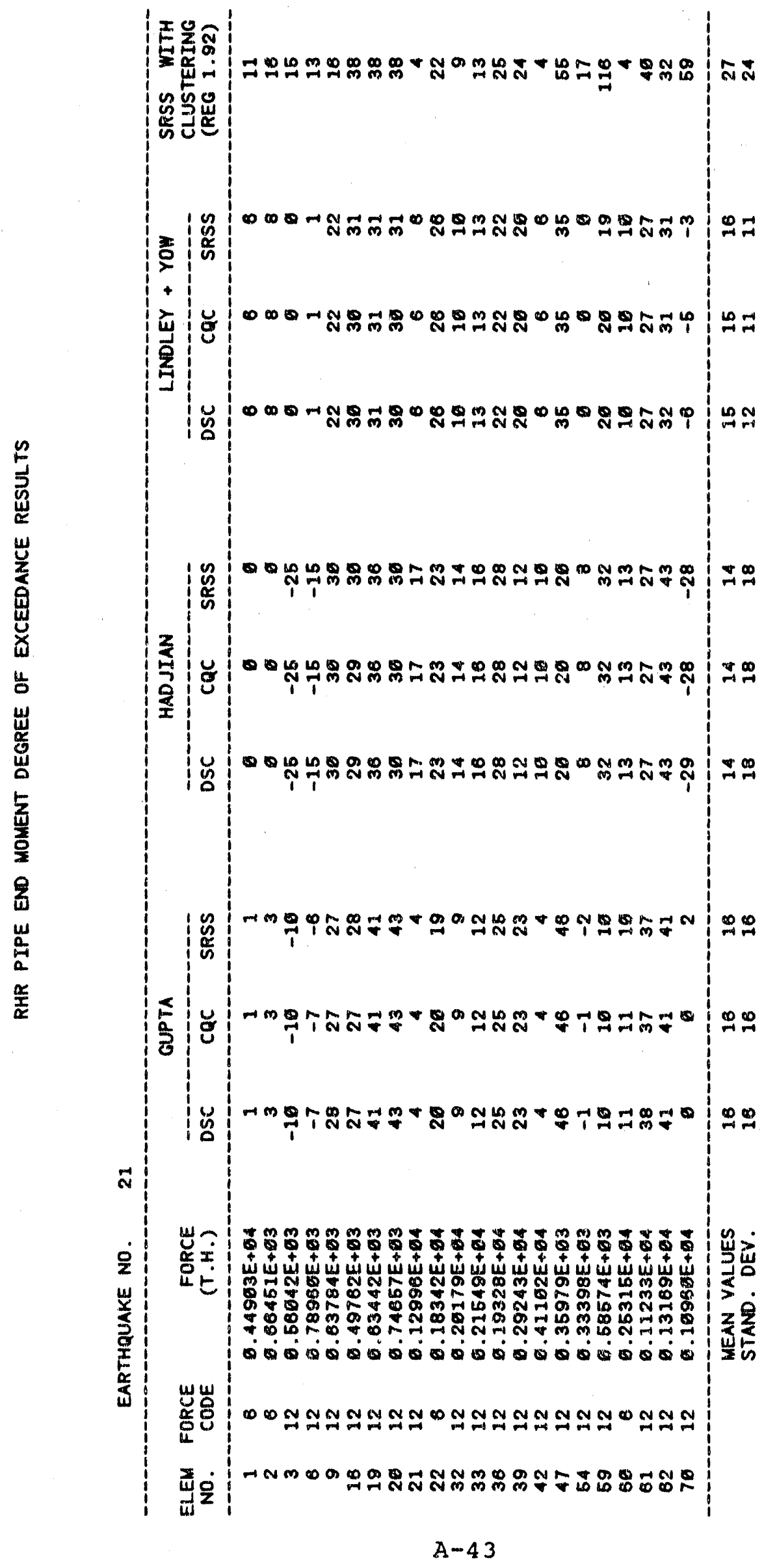




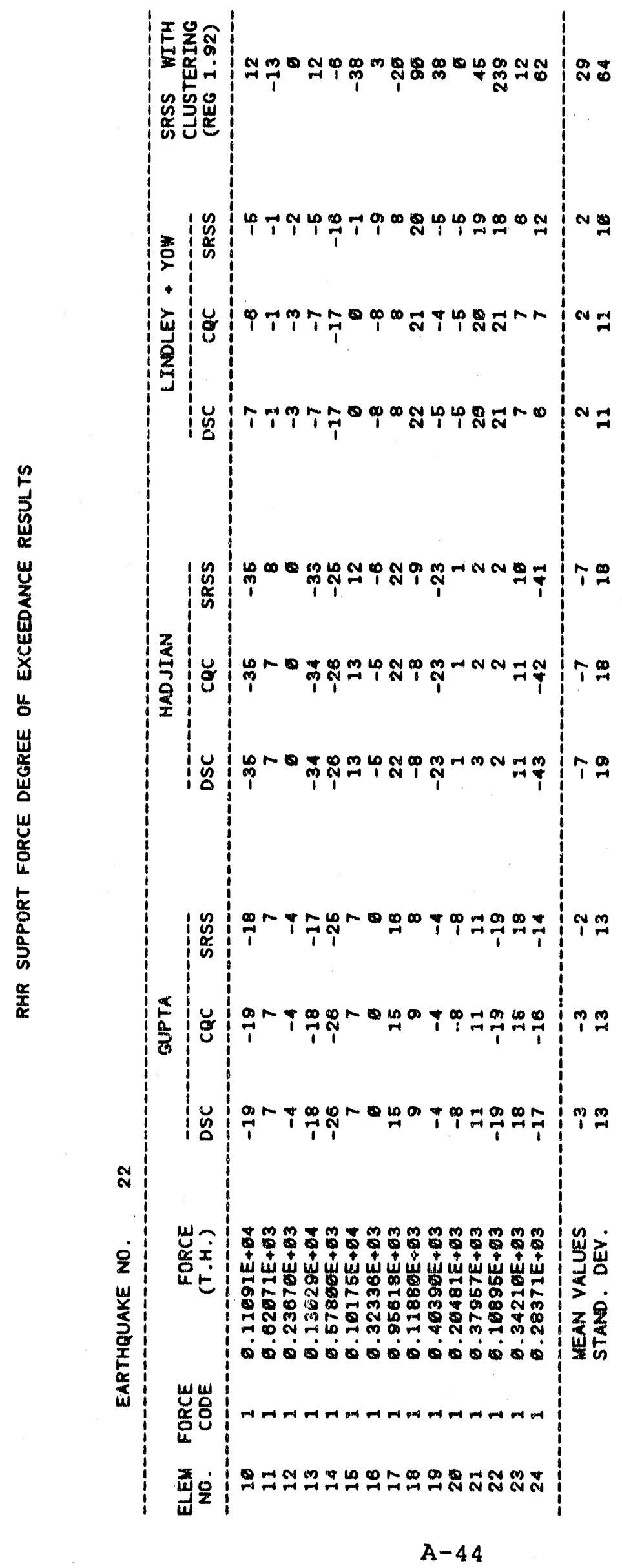




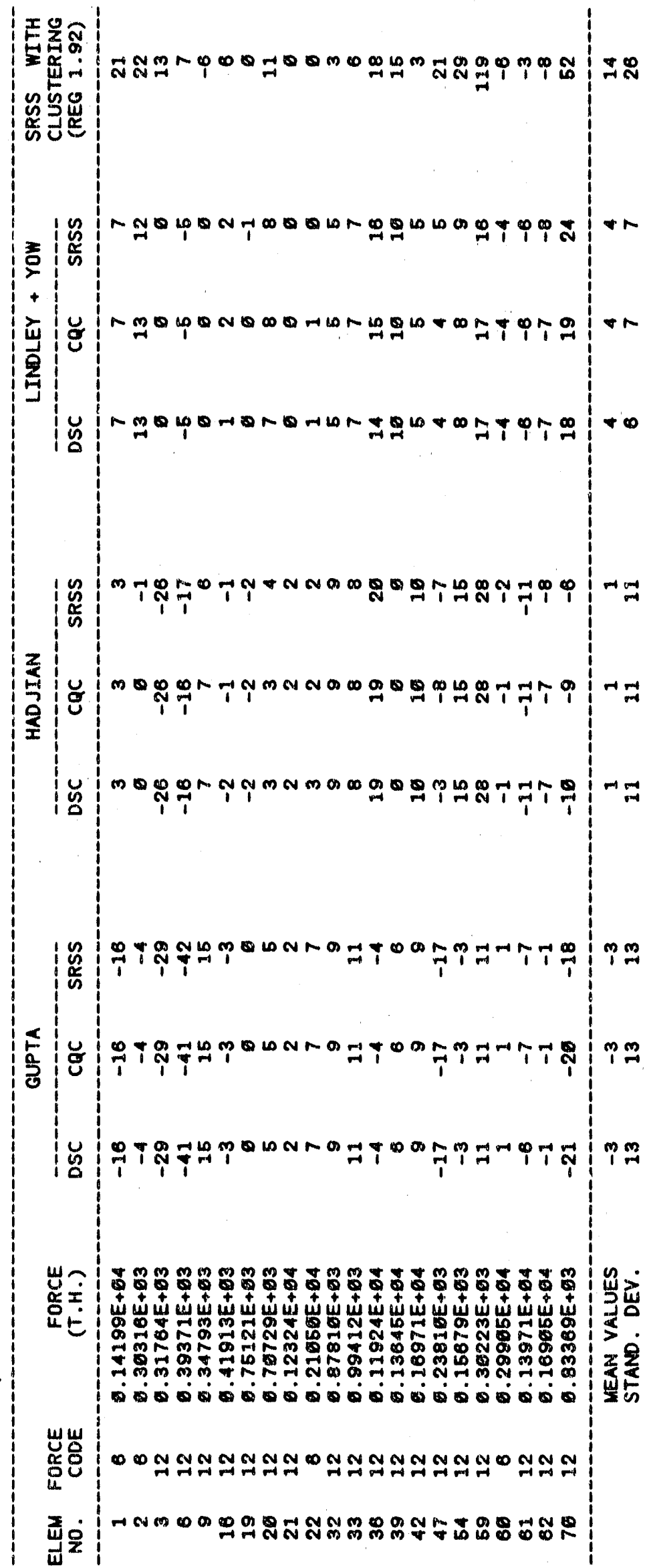

$$
\text { A-57 }
$$




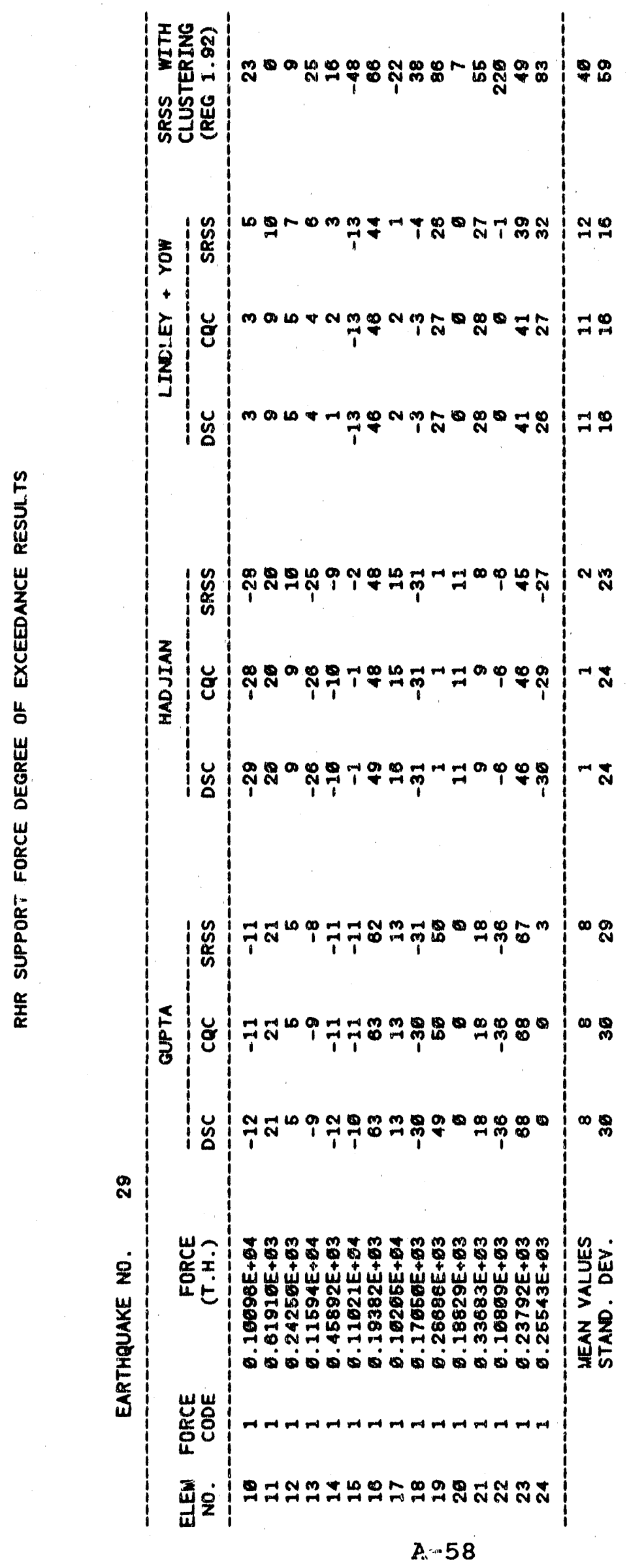




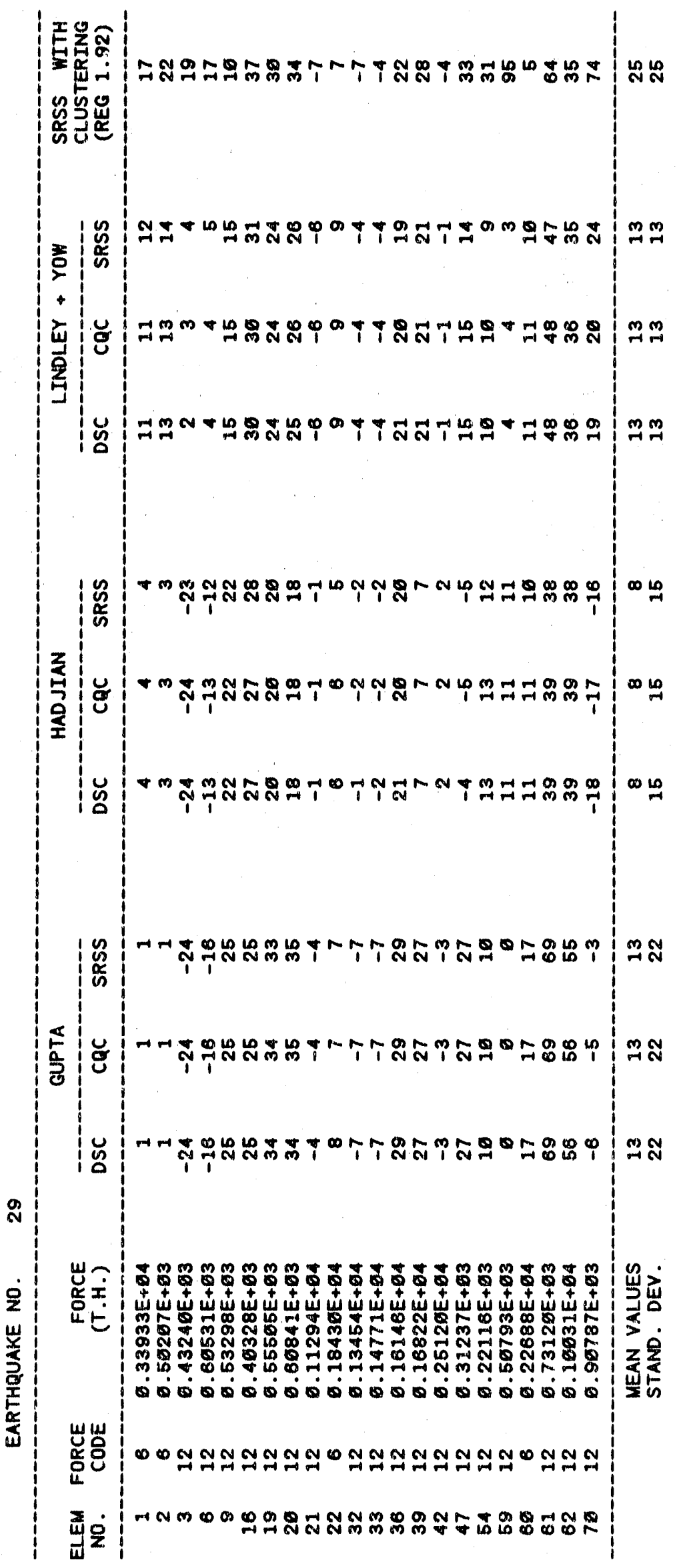

$$
\text { A-59 }
$$




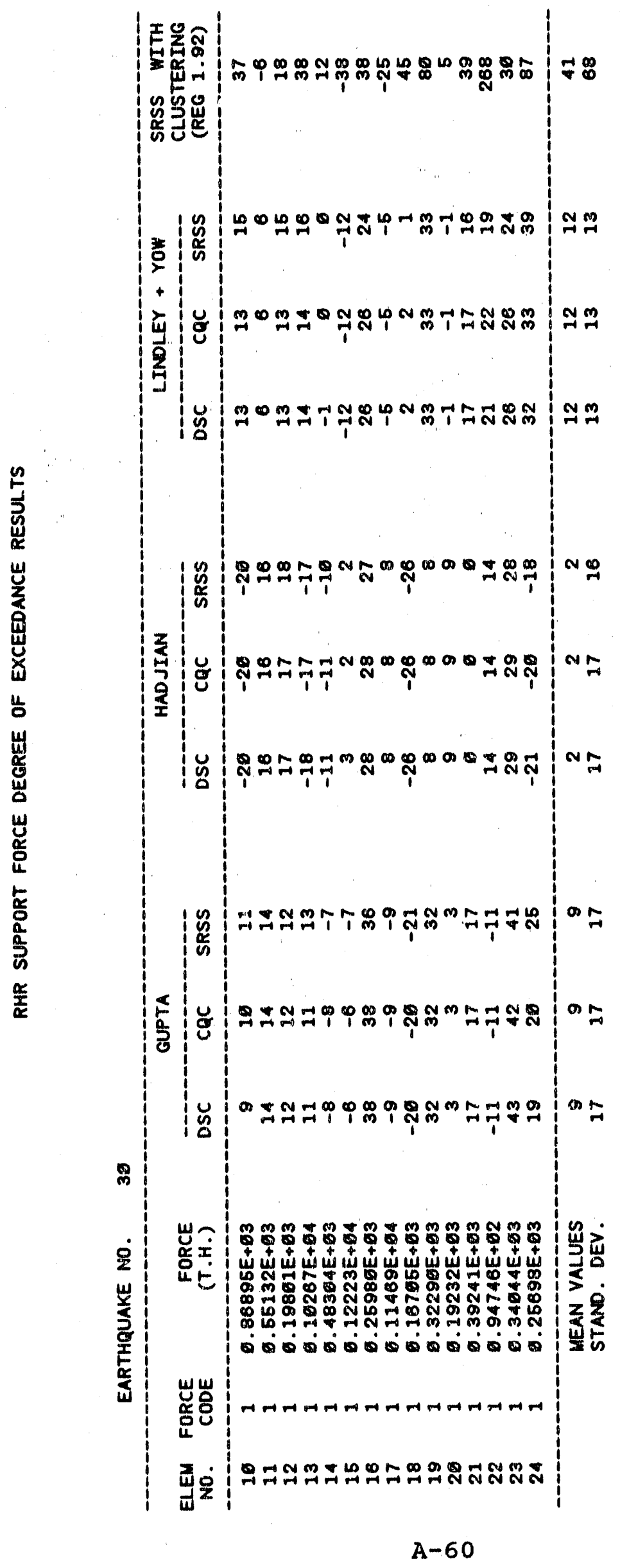




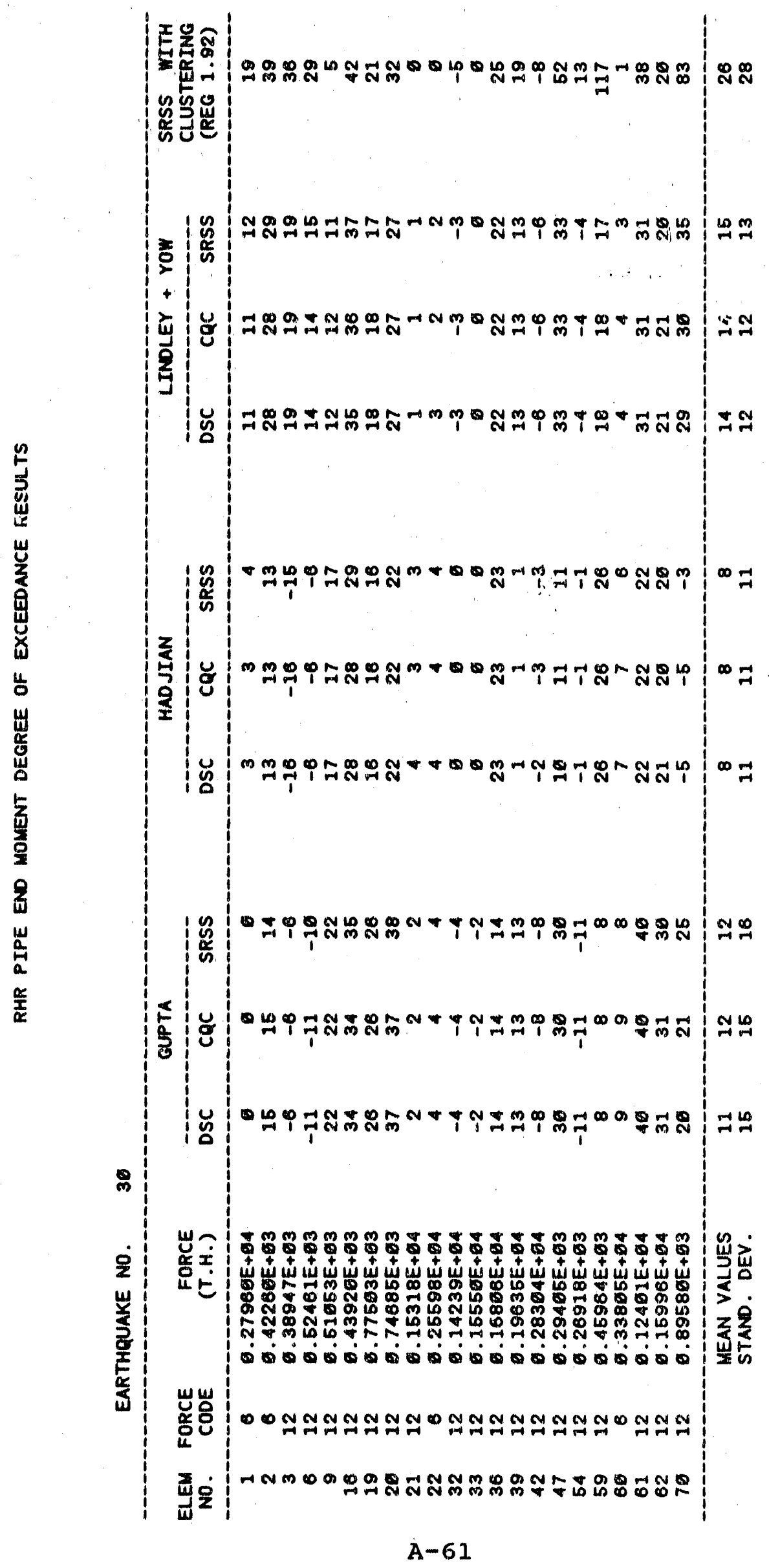




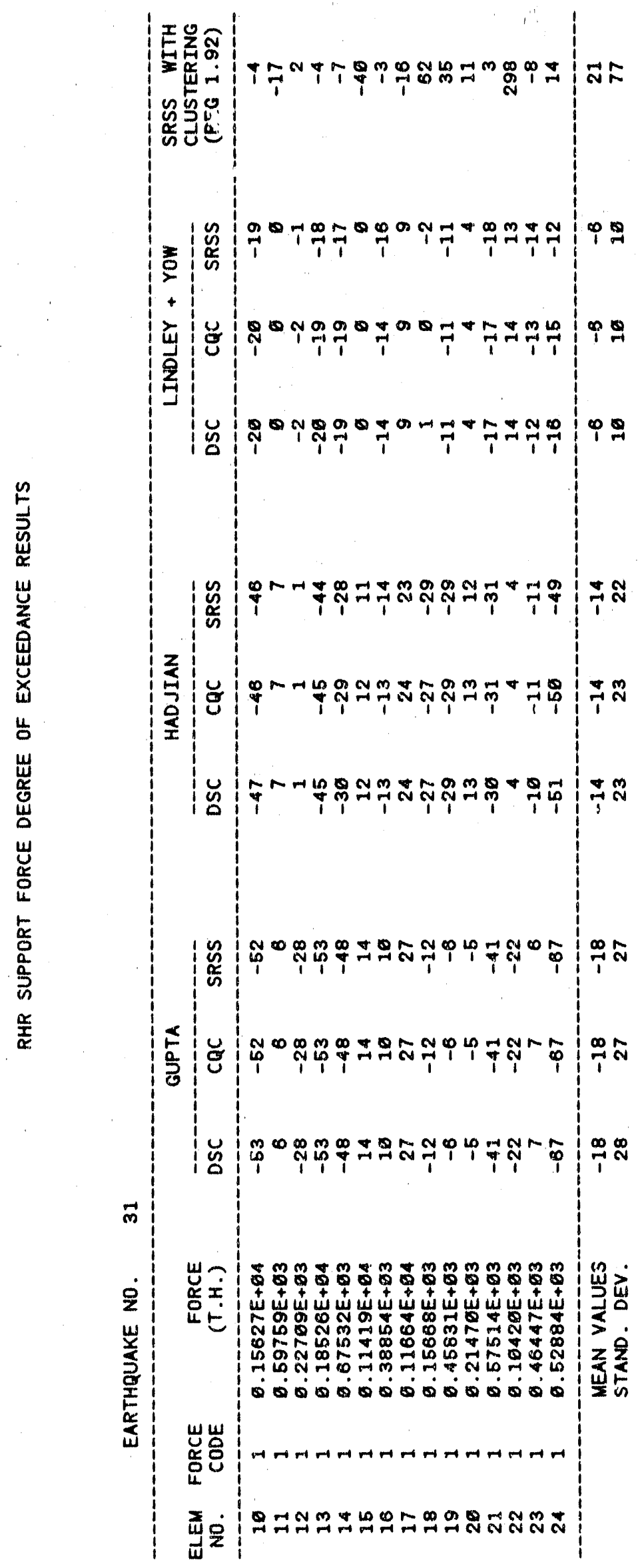

A-62 


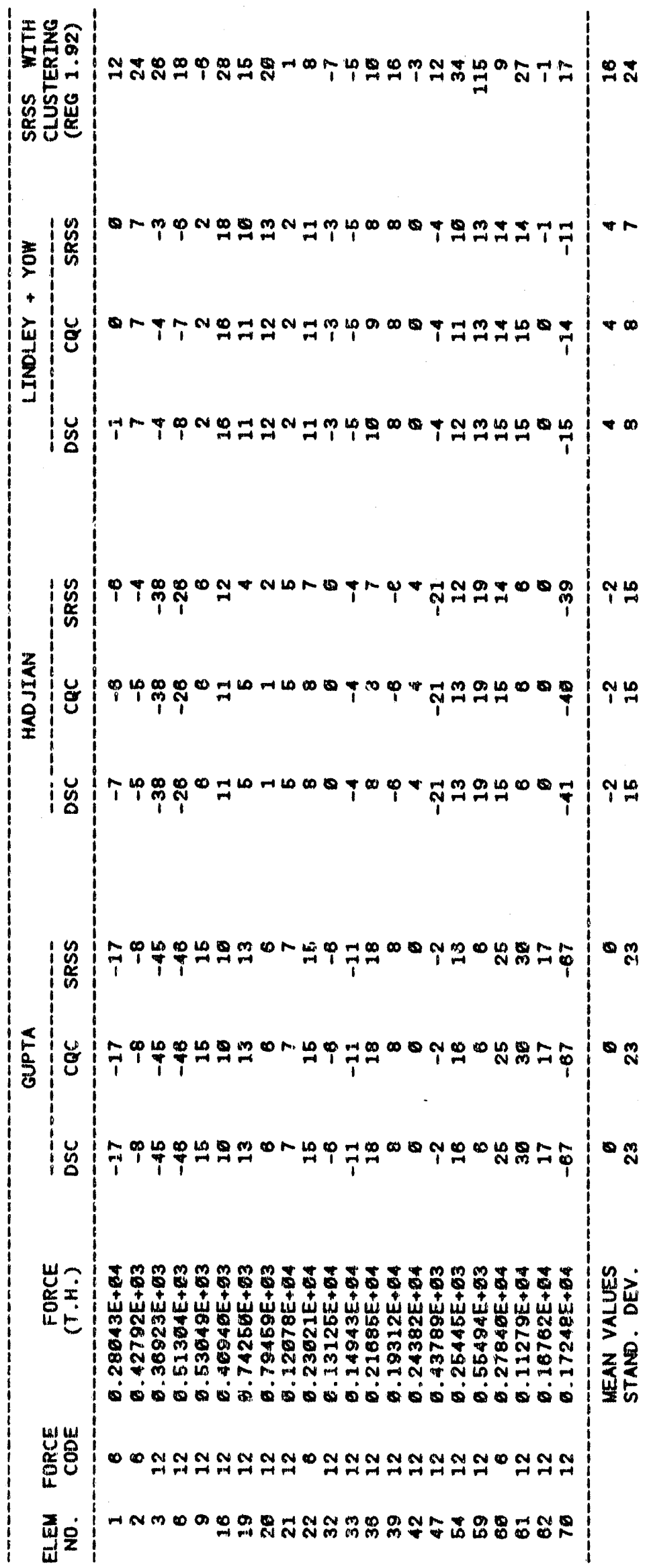

$$
\text { A }-63
$$




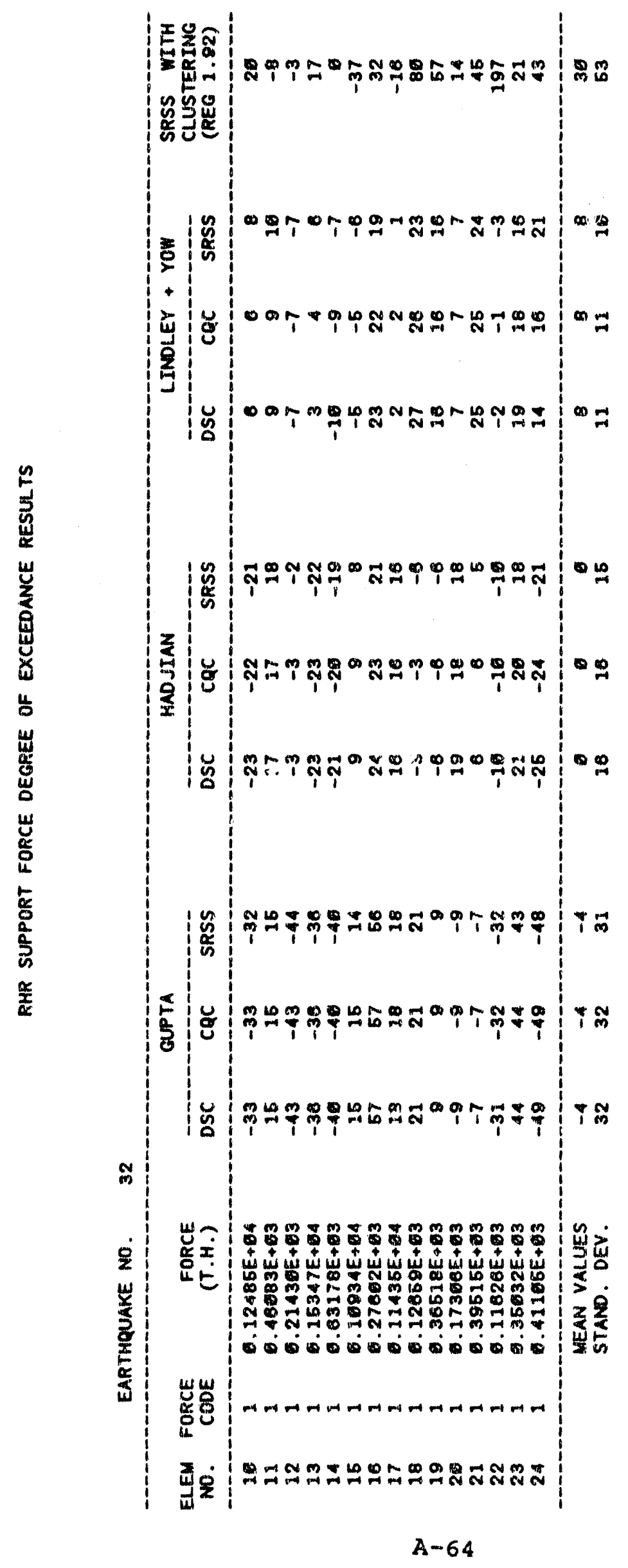




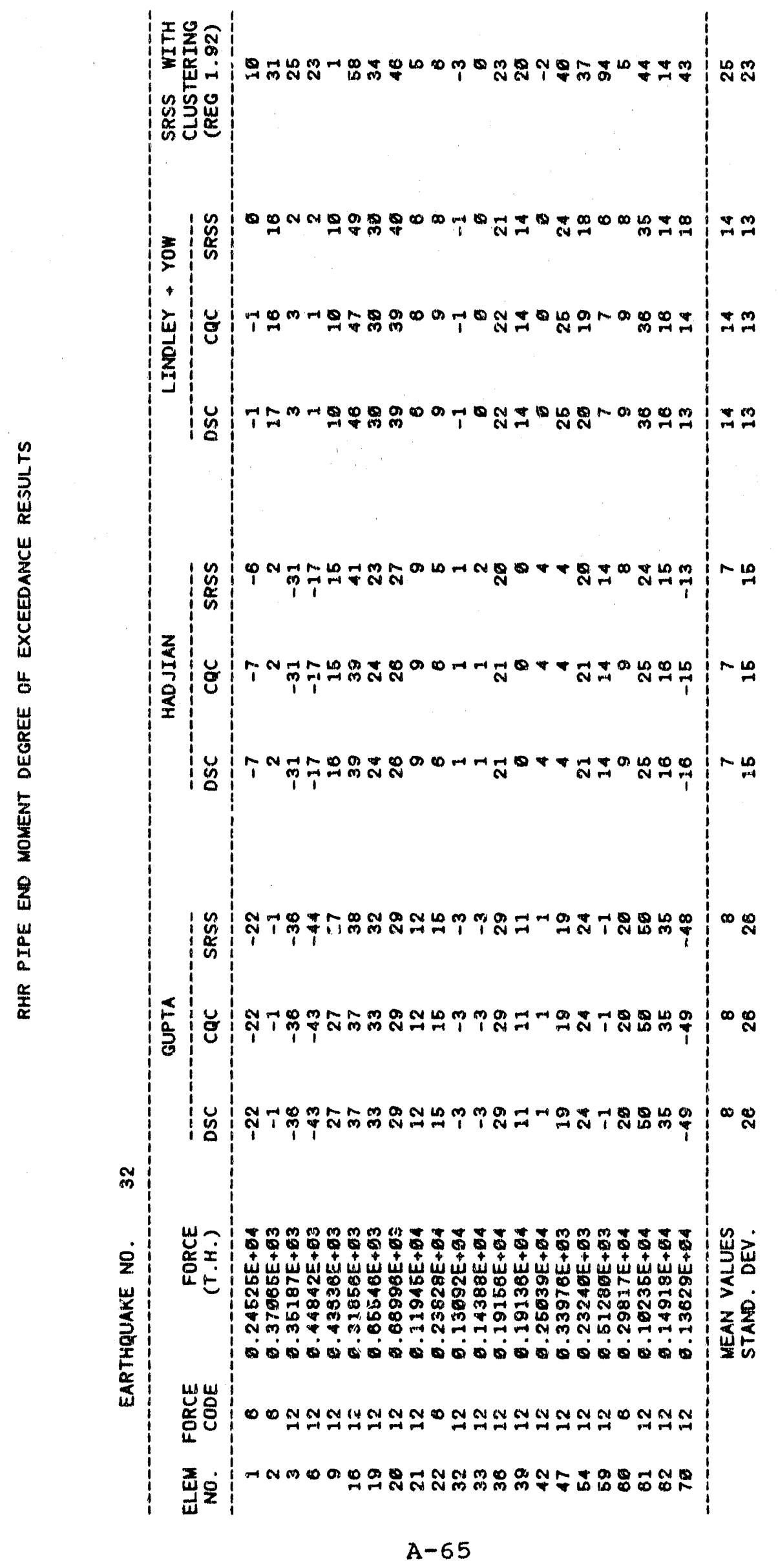




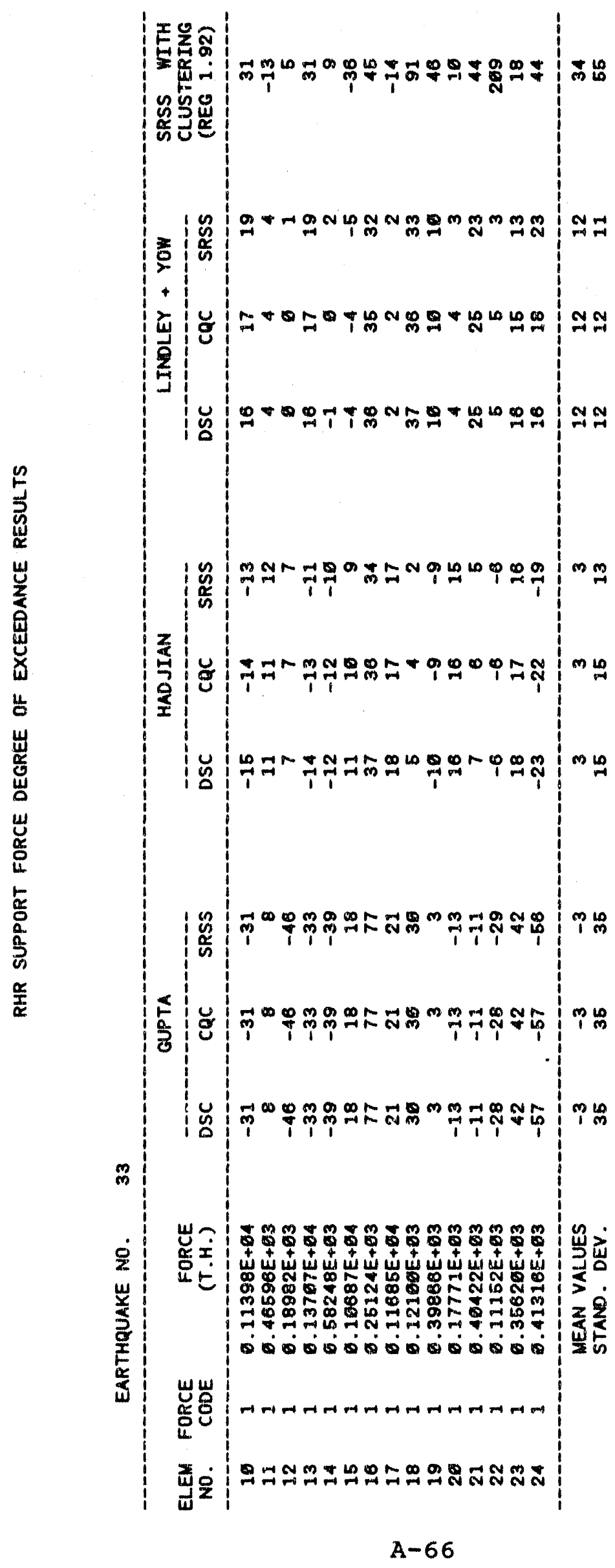




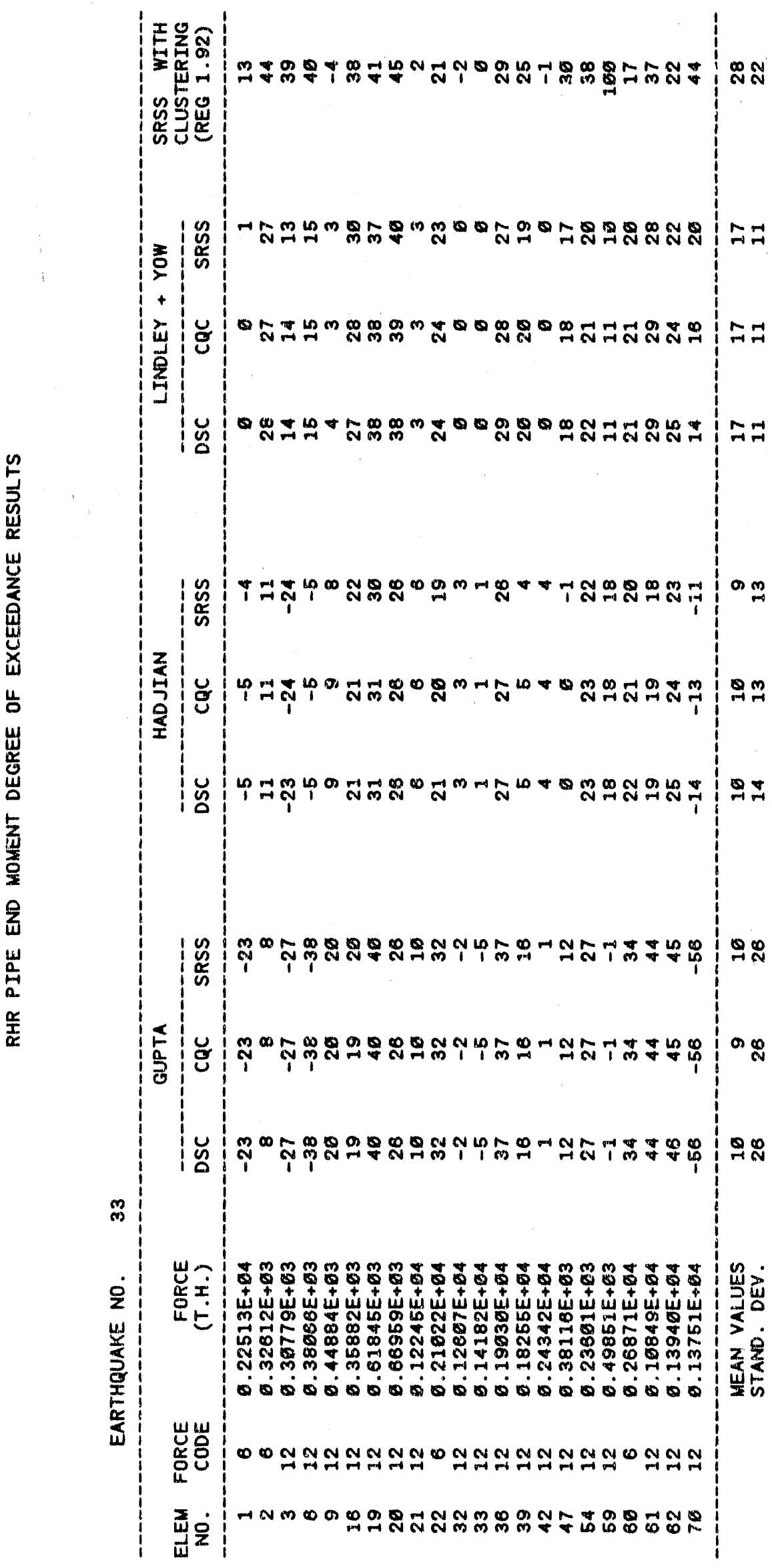

A-67 


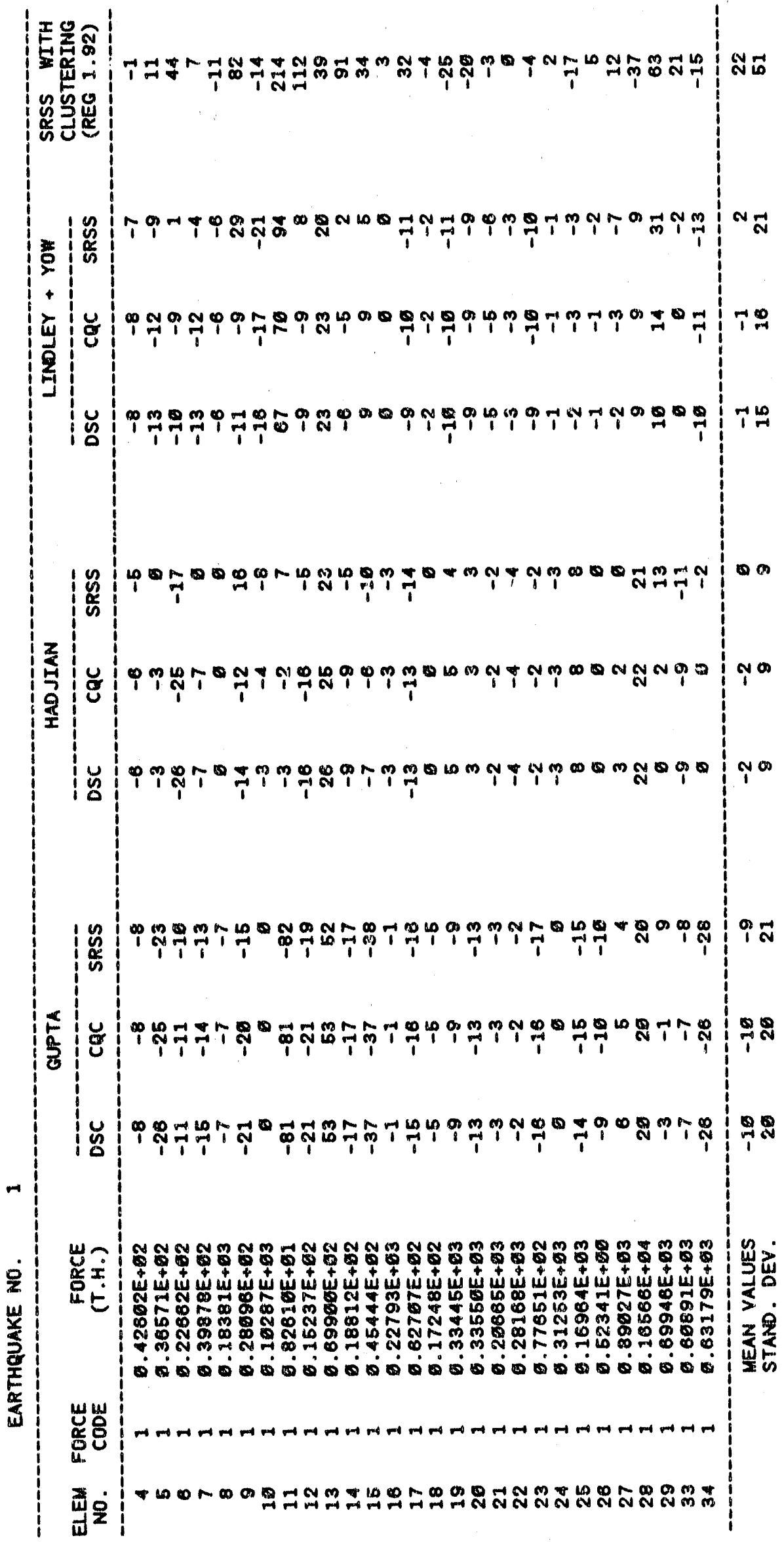




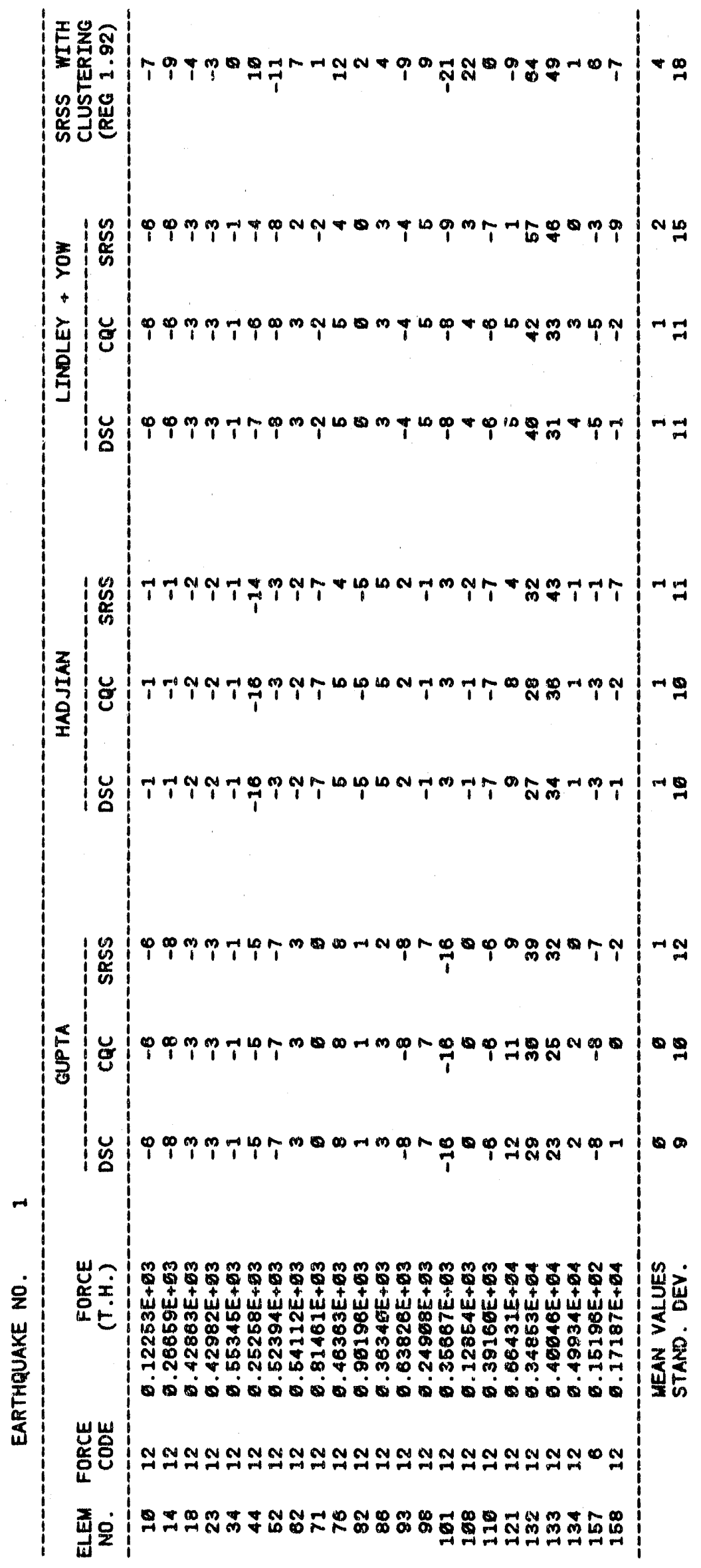




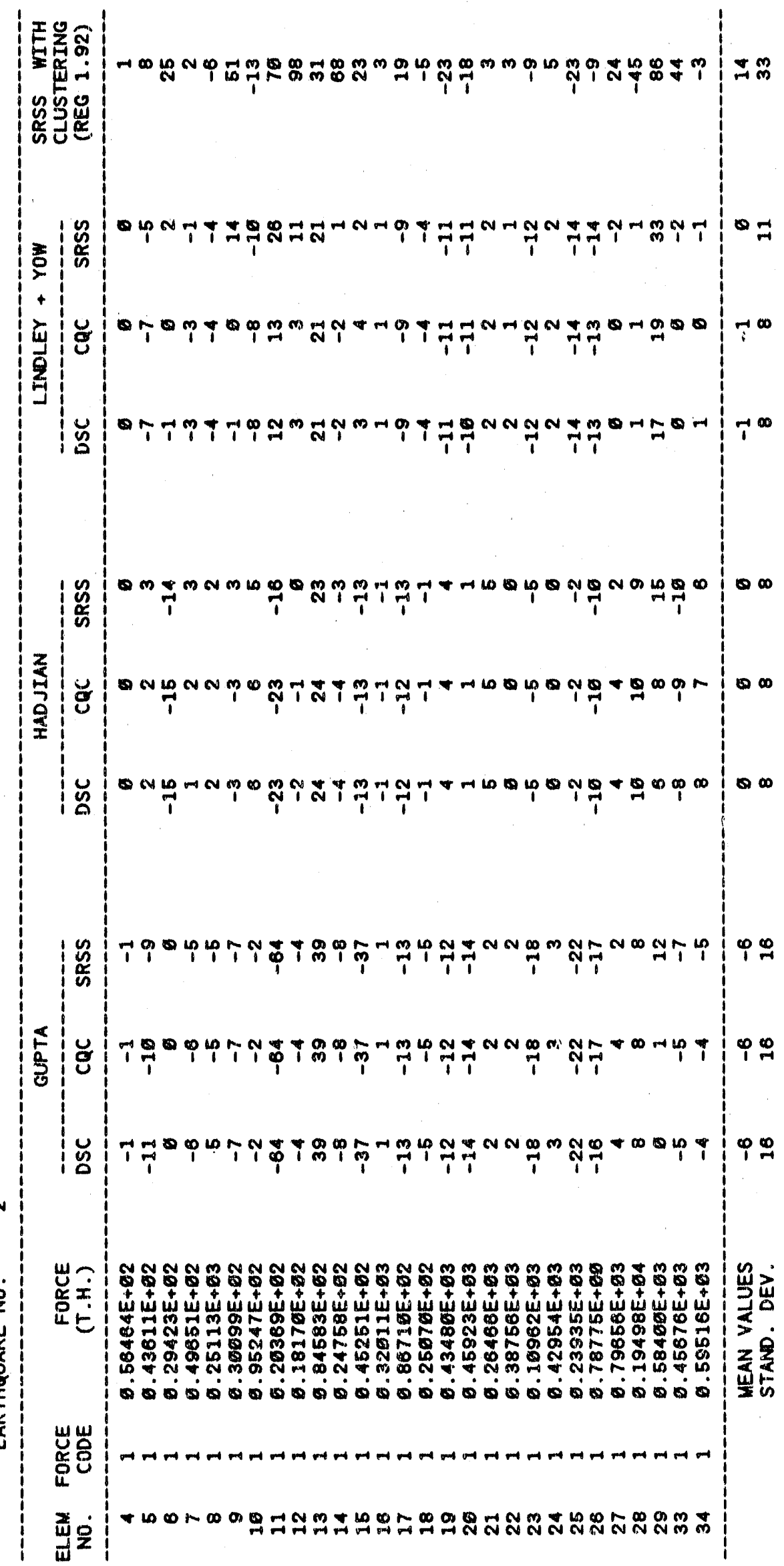




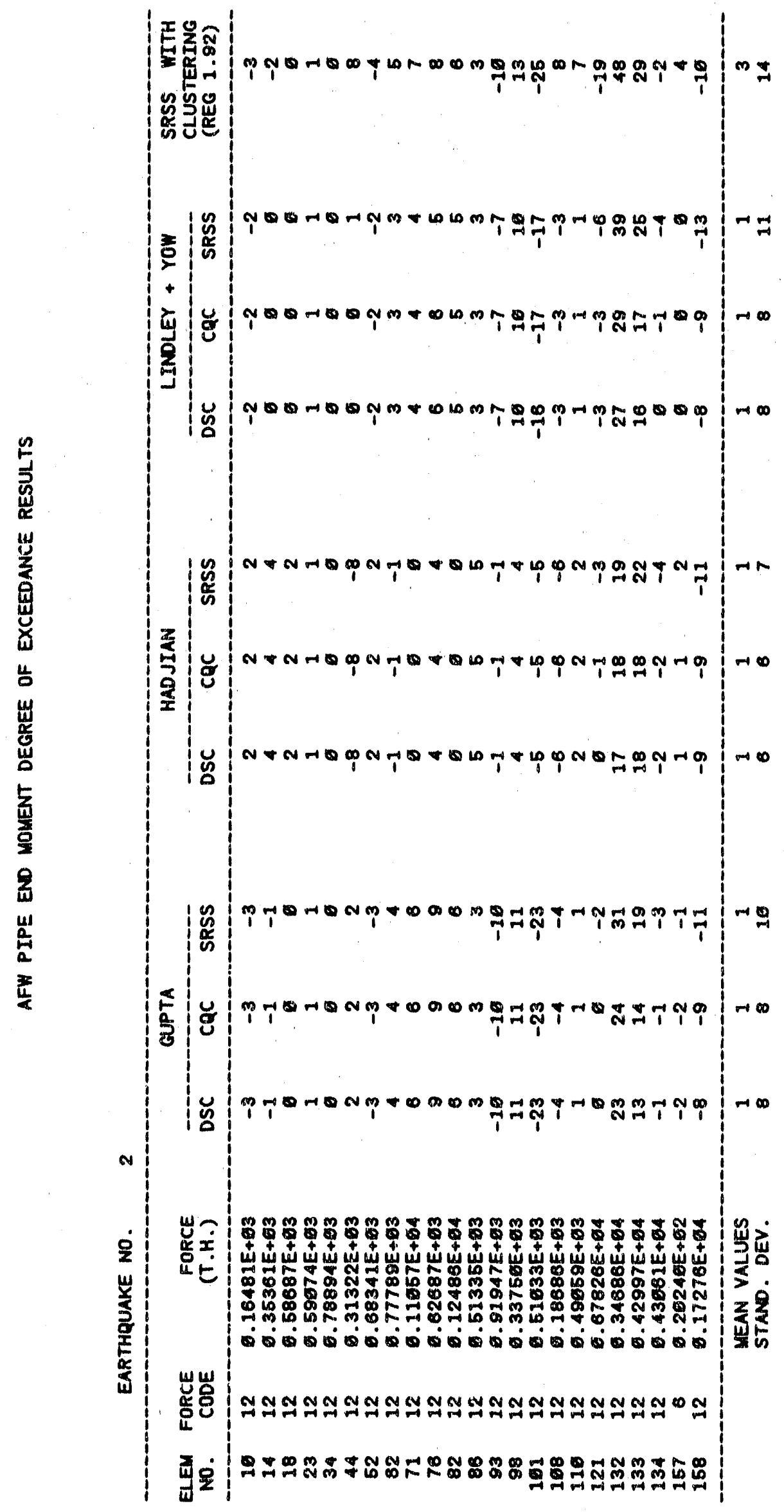




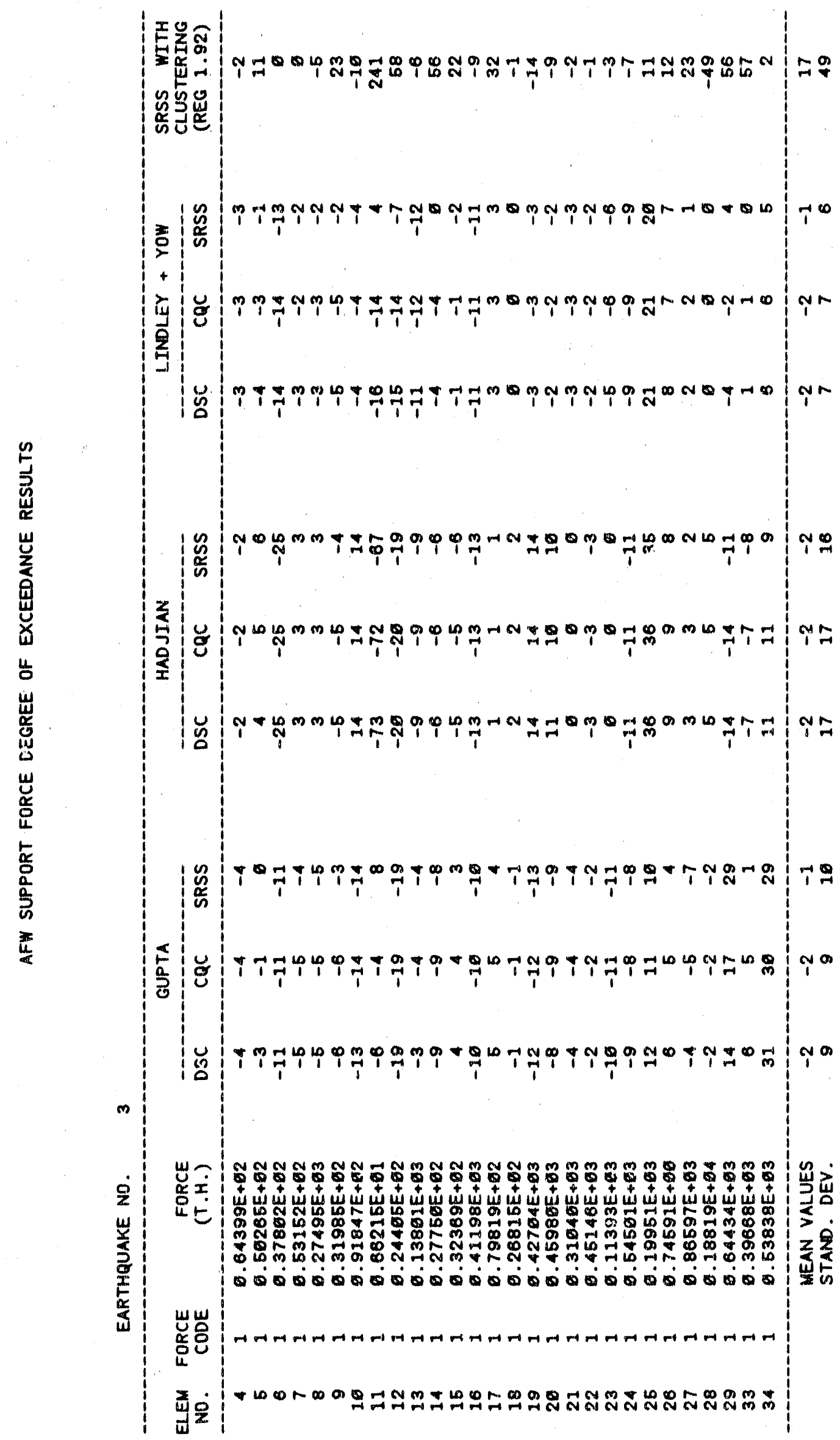




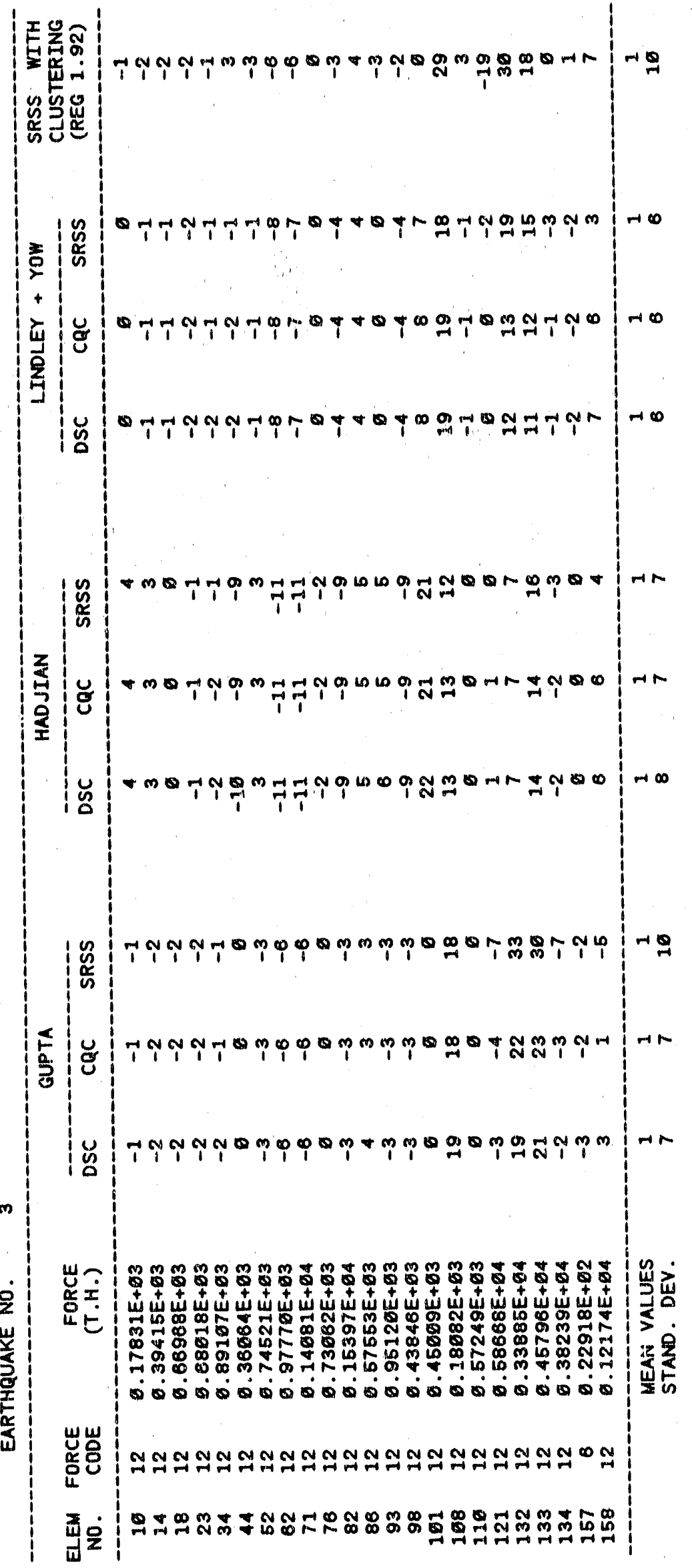




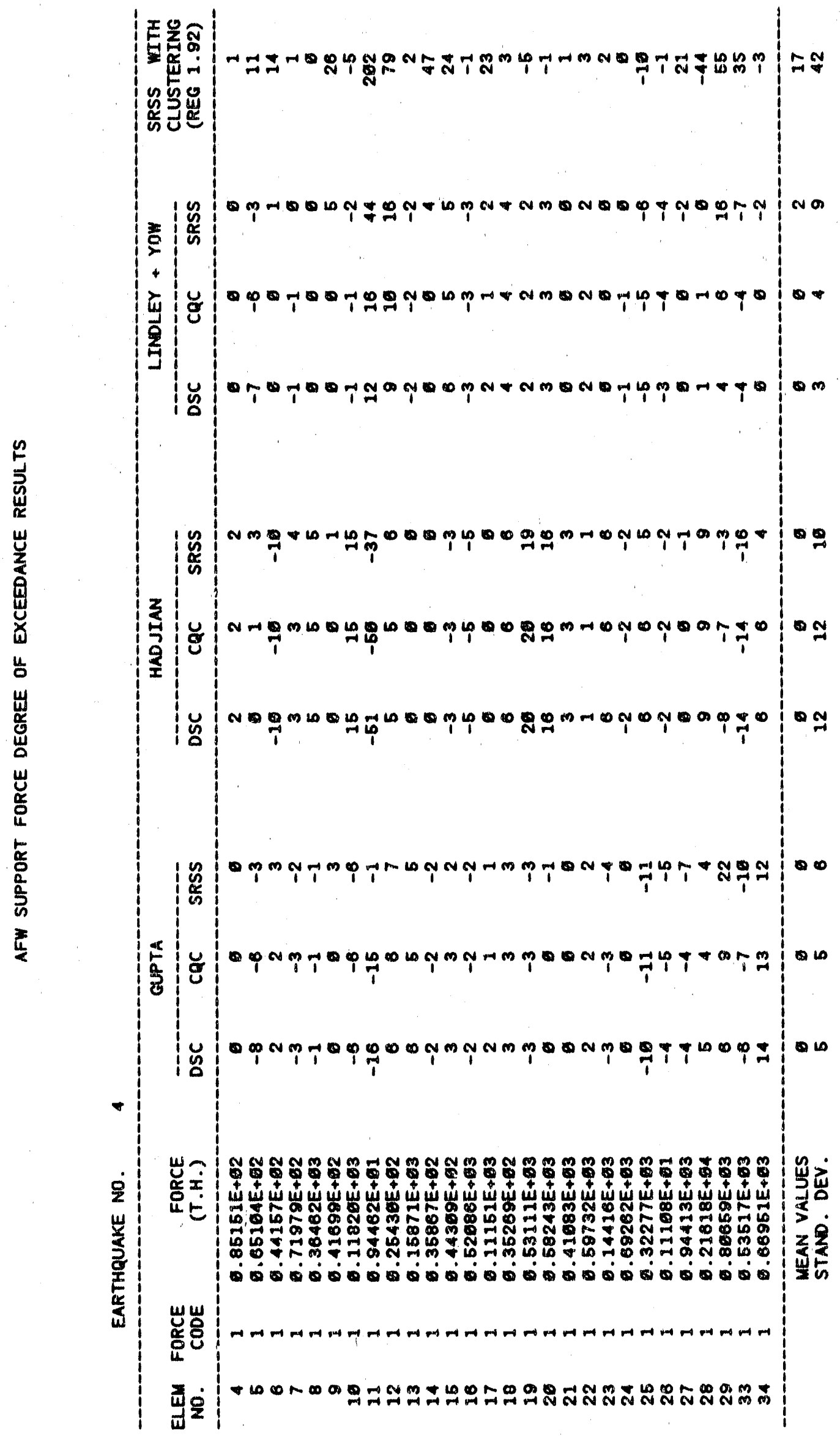




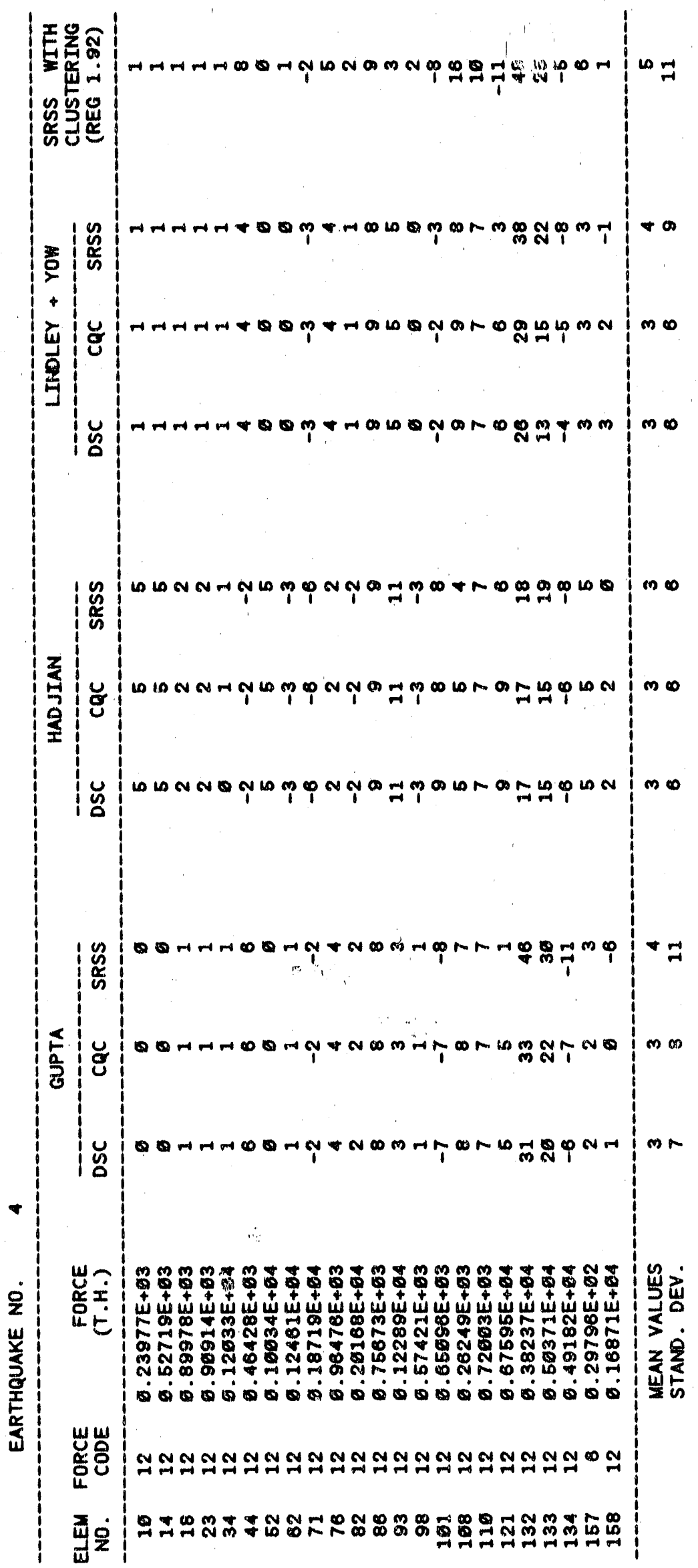

A-75 


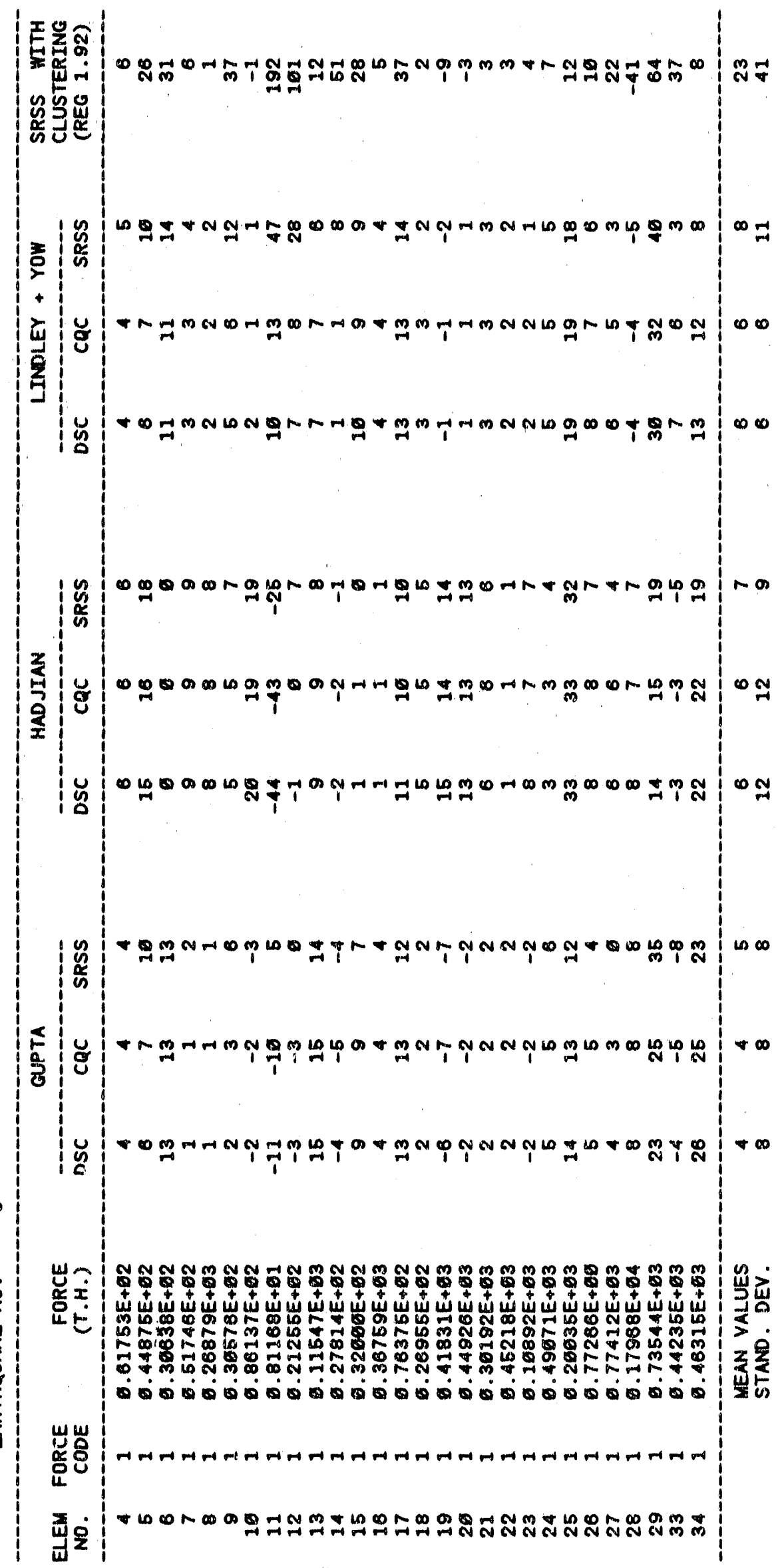

$$
\text { A }-76
$$




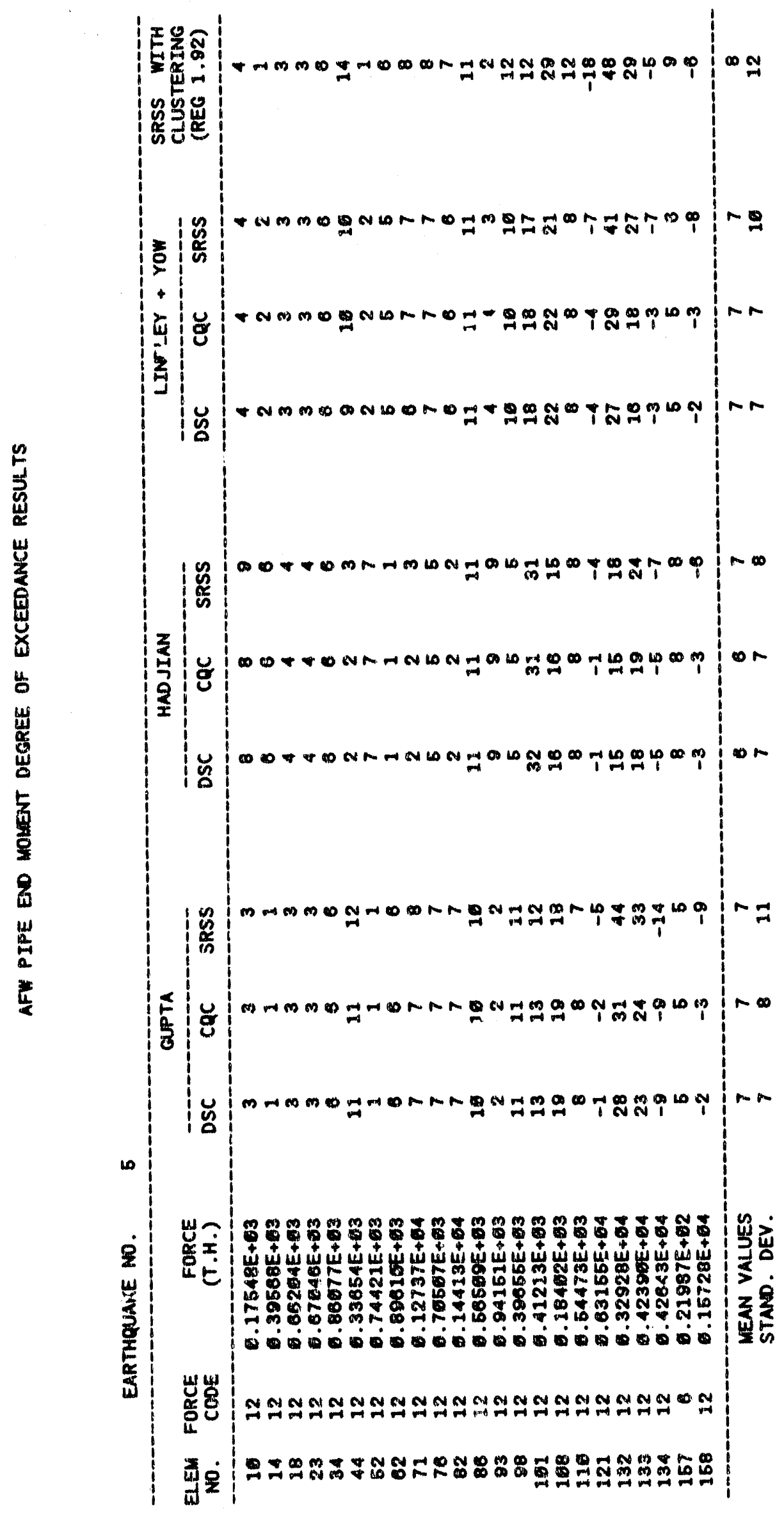




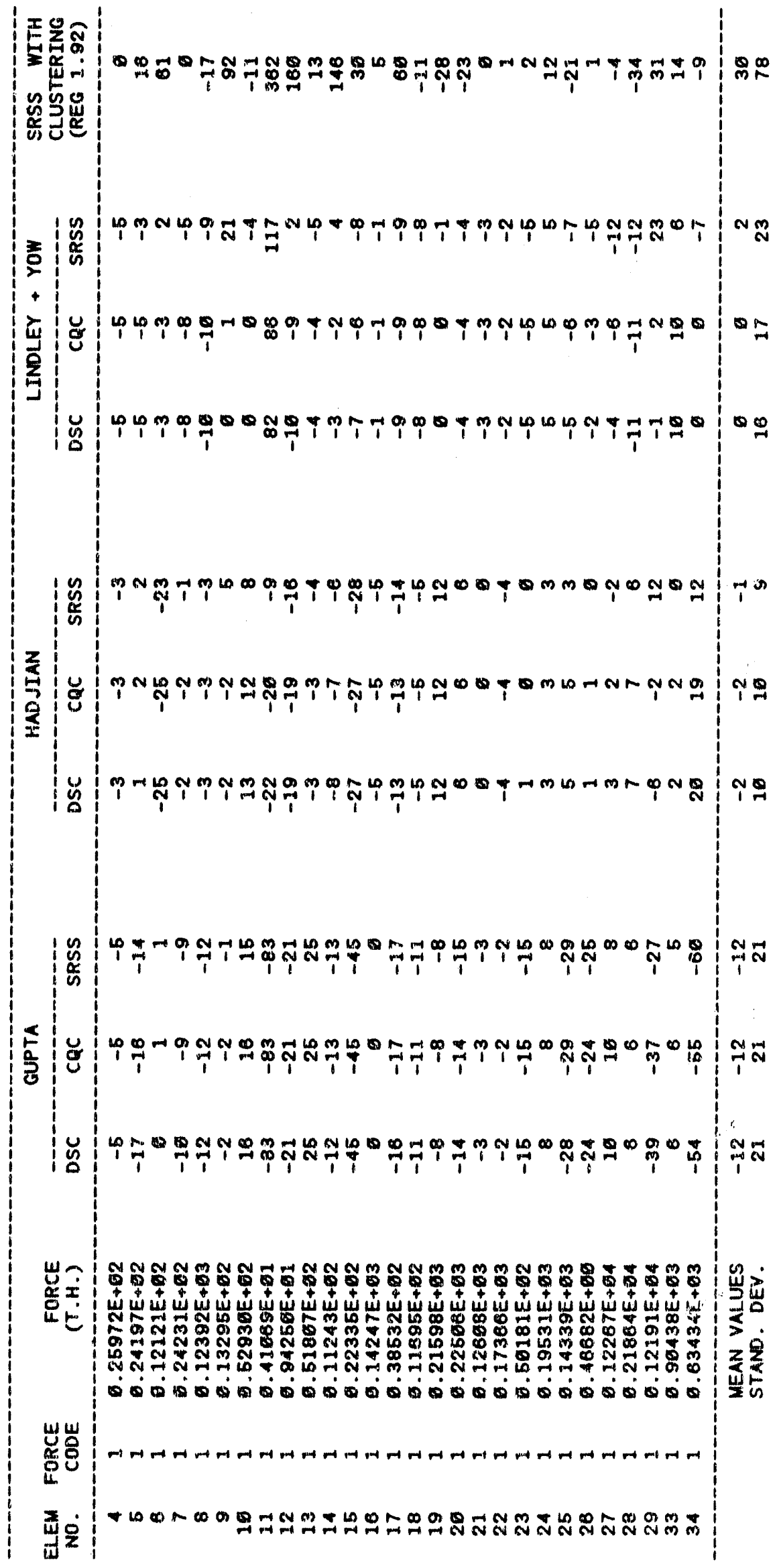




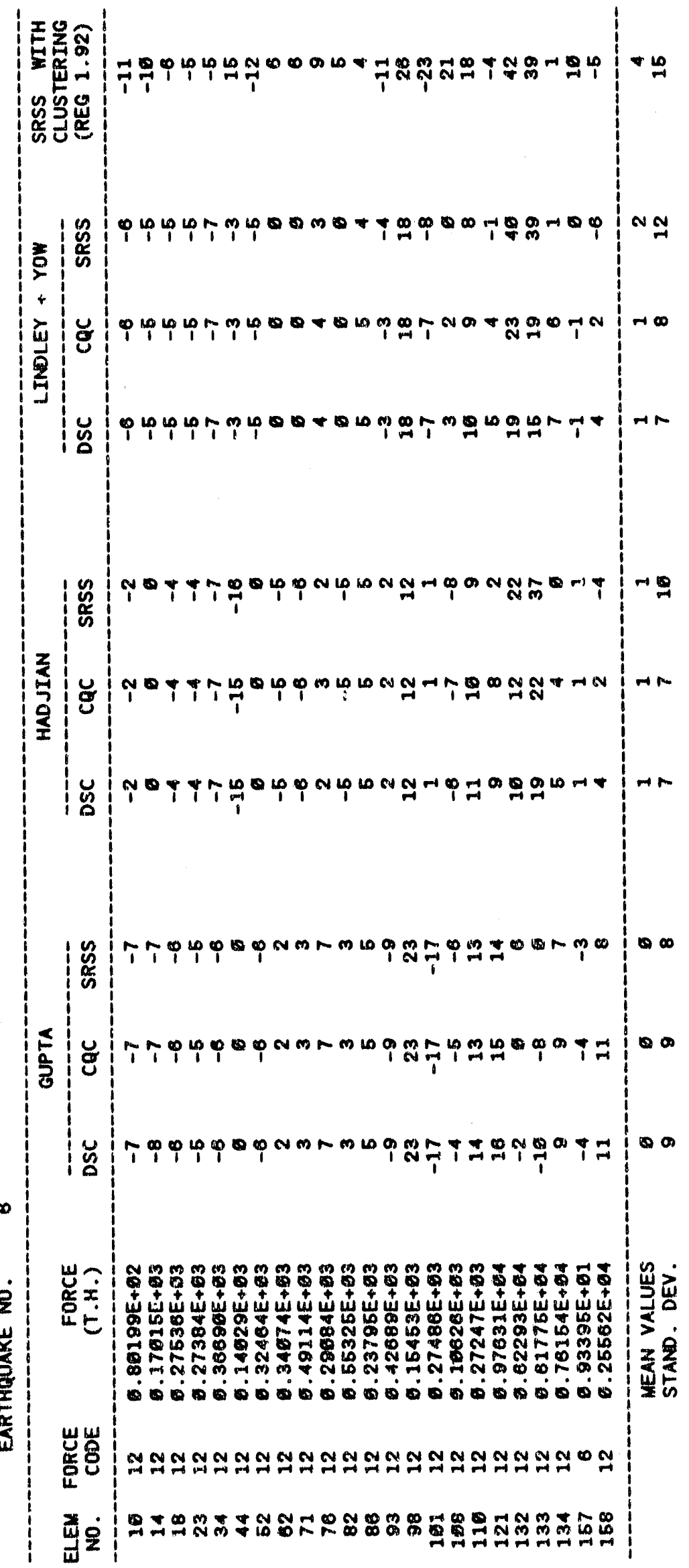

$$
\text { n-73 }
$$




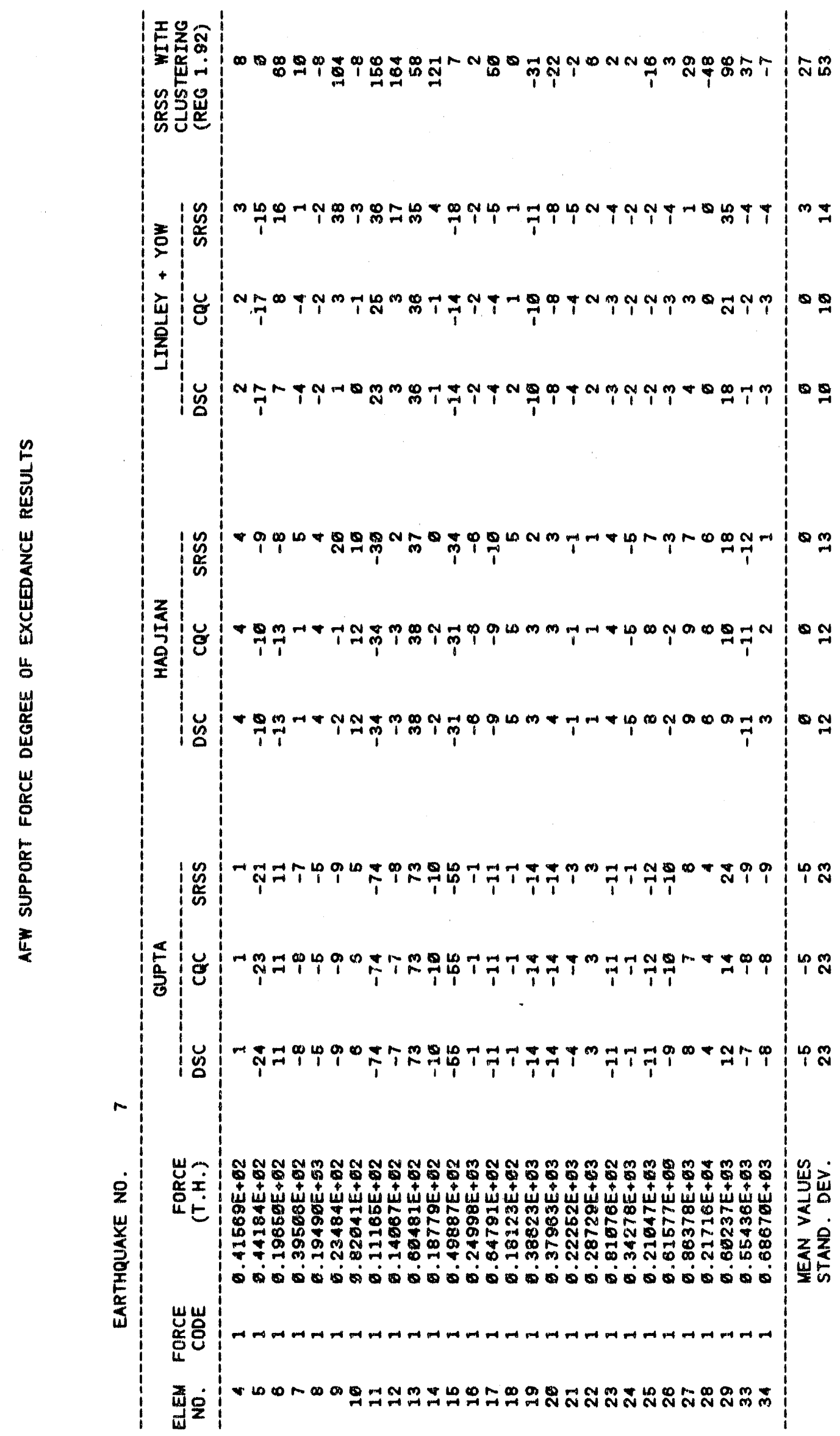




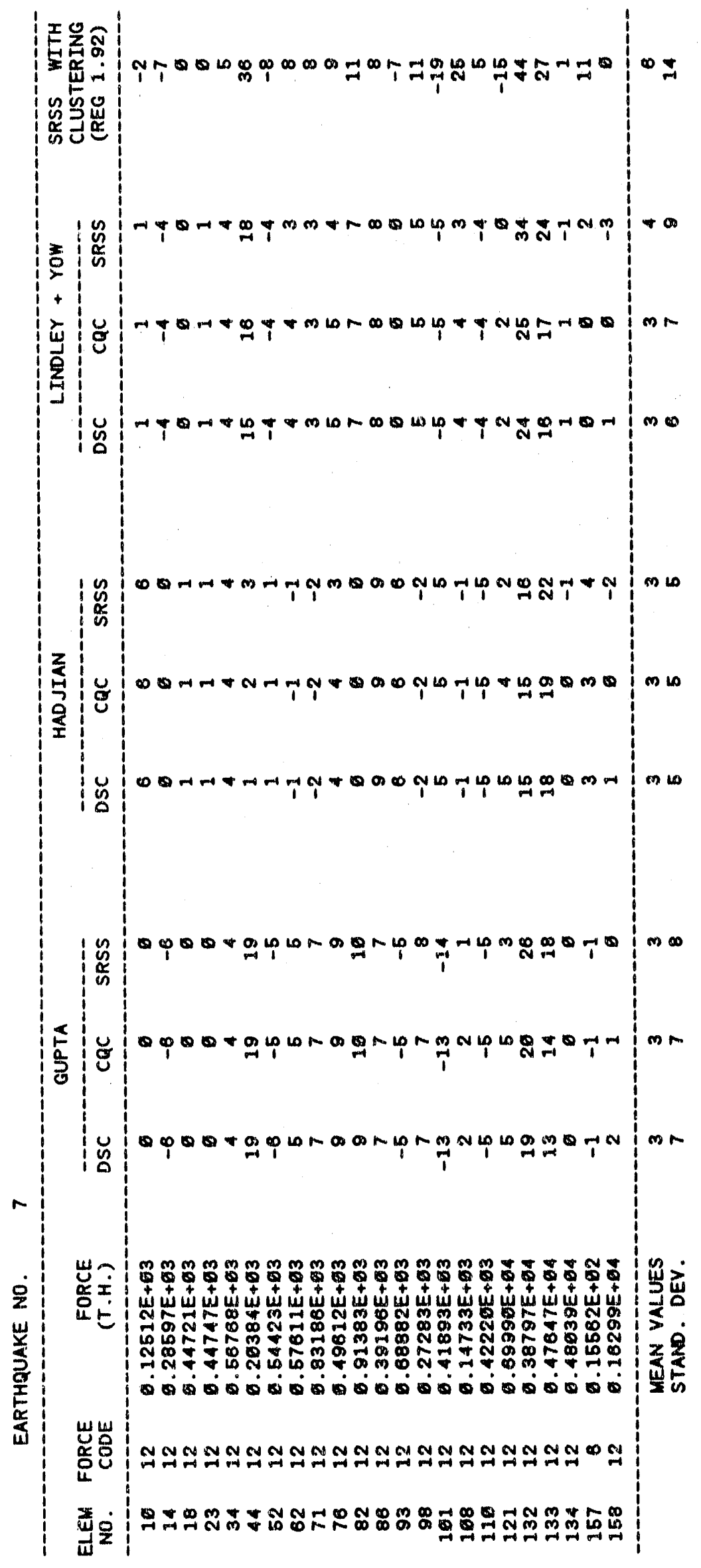




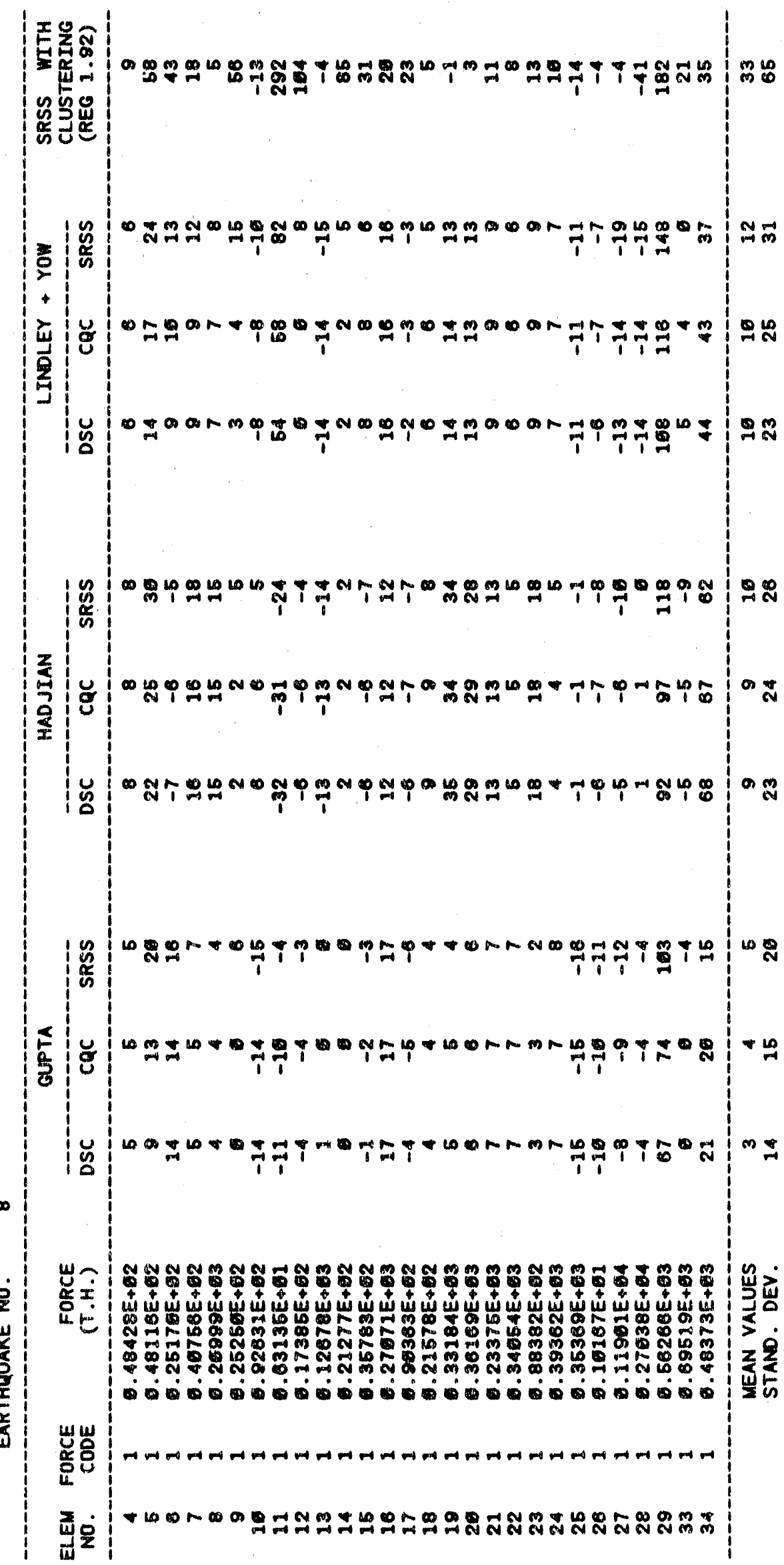




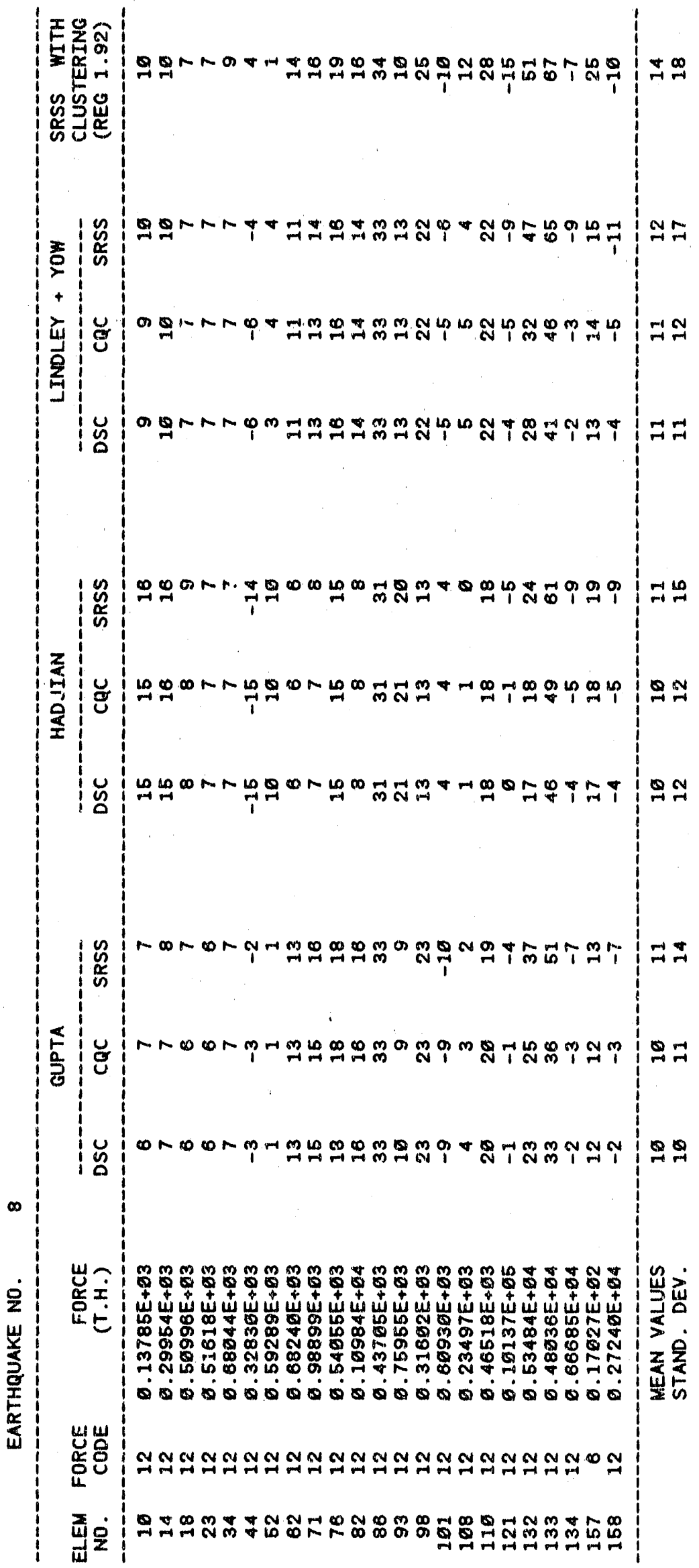




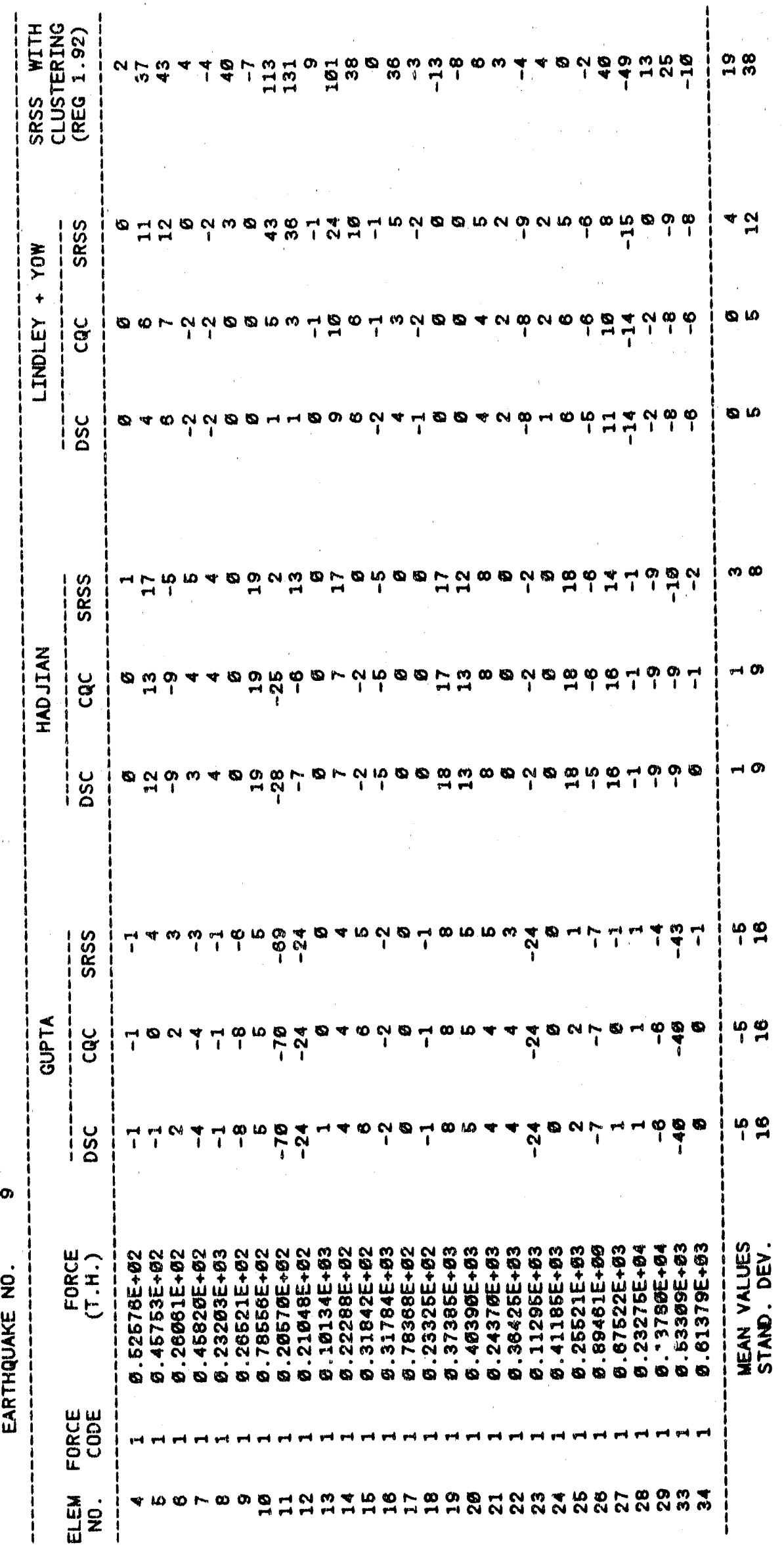




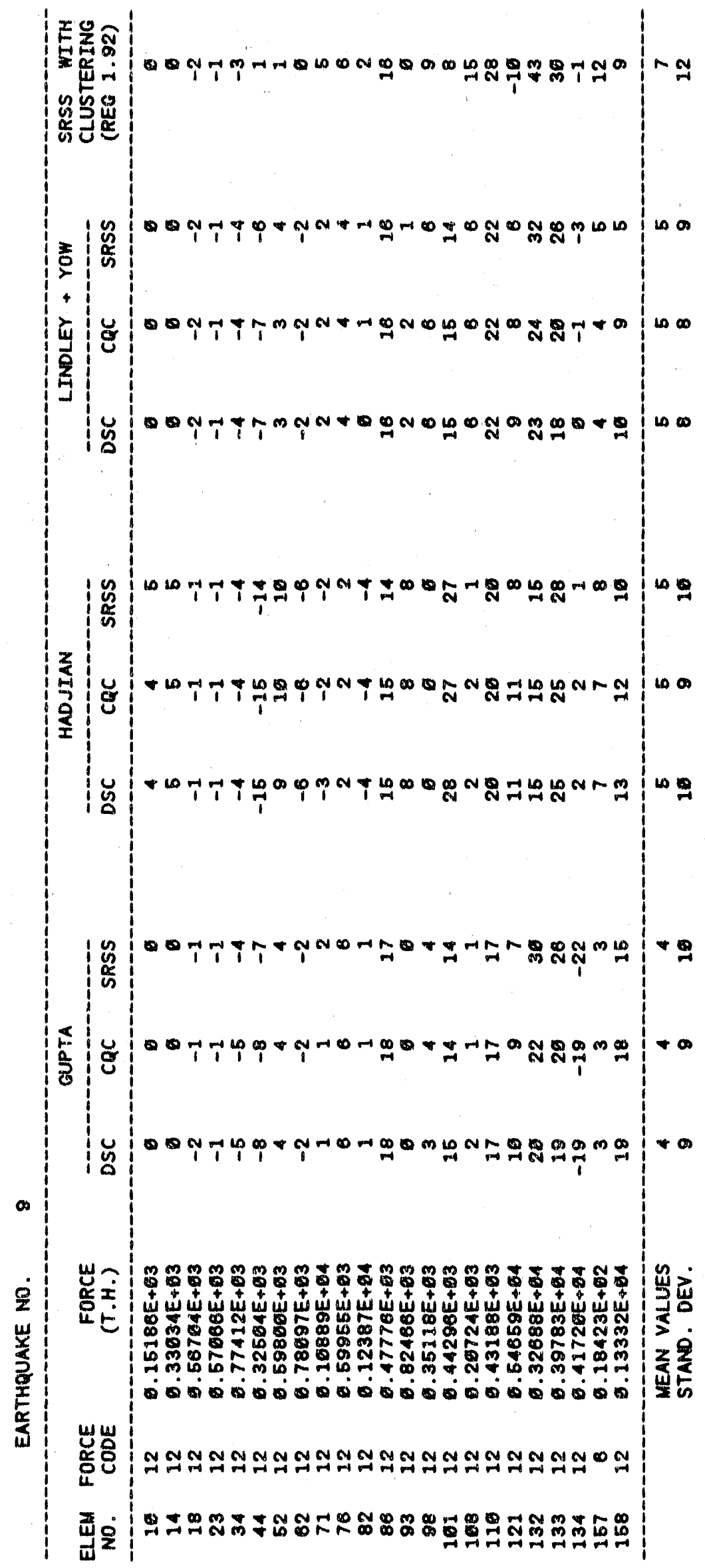

$$
\text { A }-85
$$




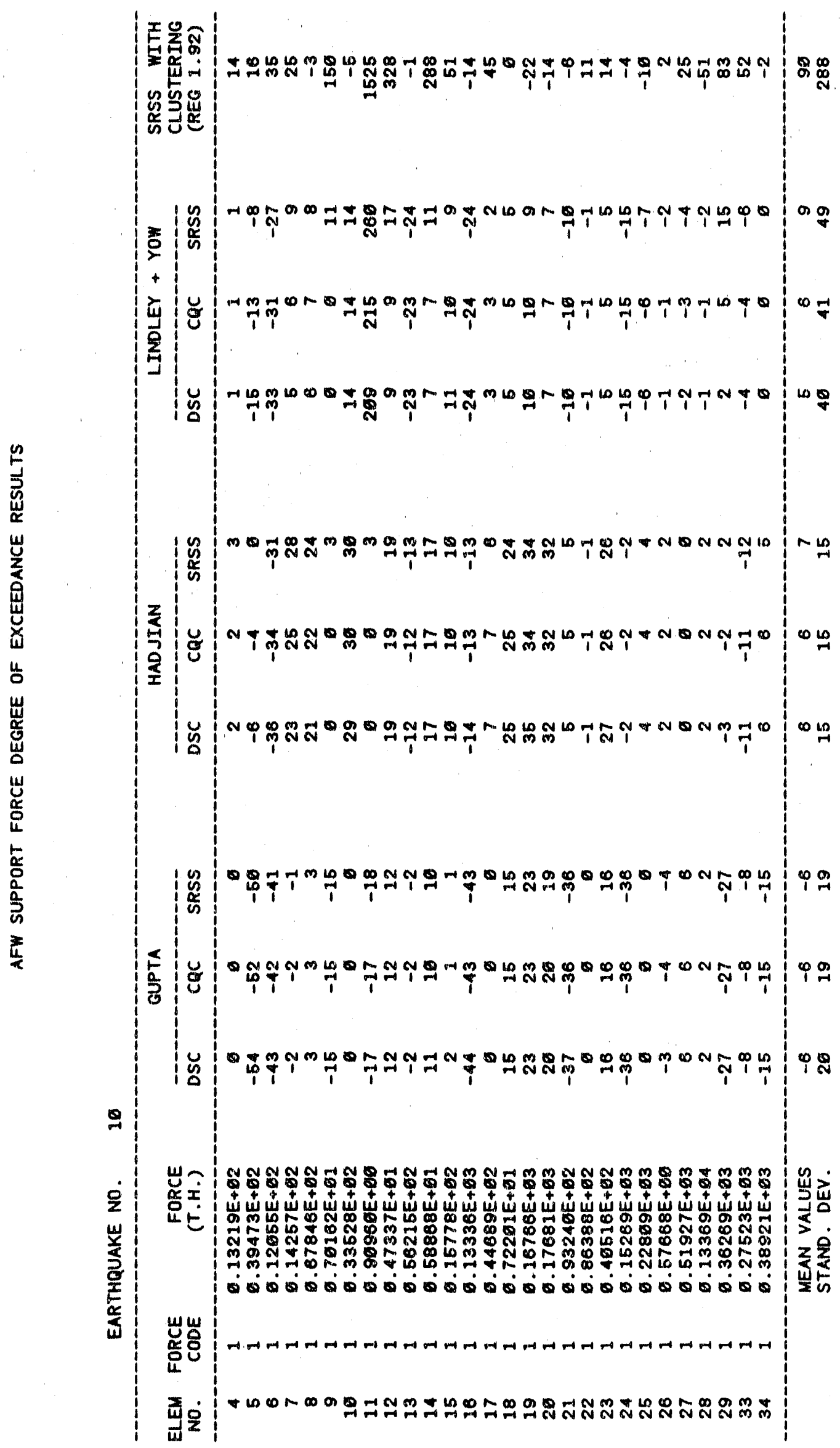




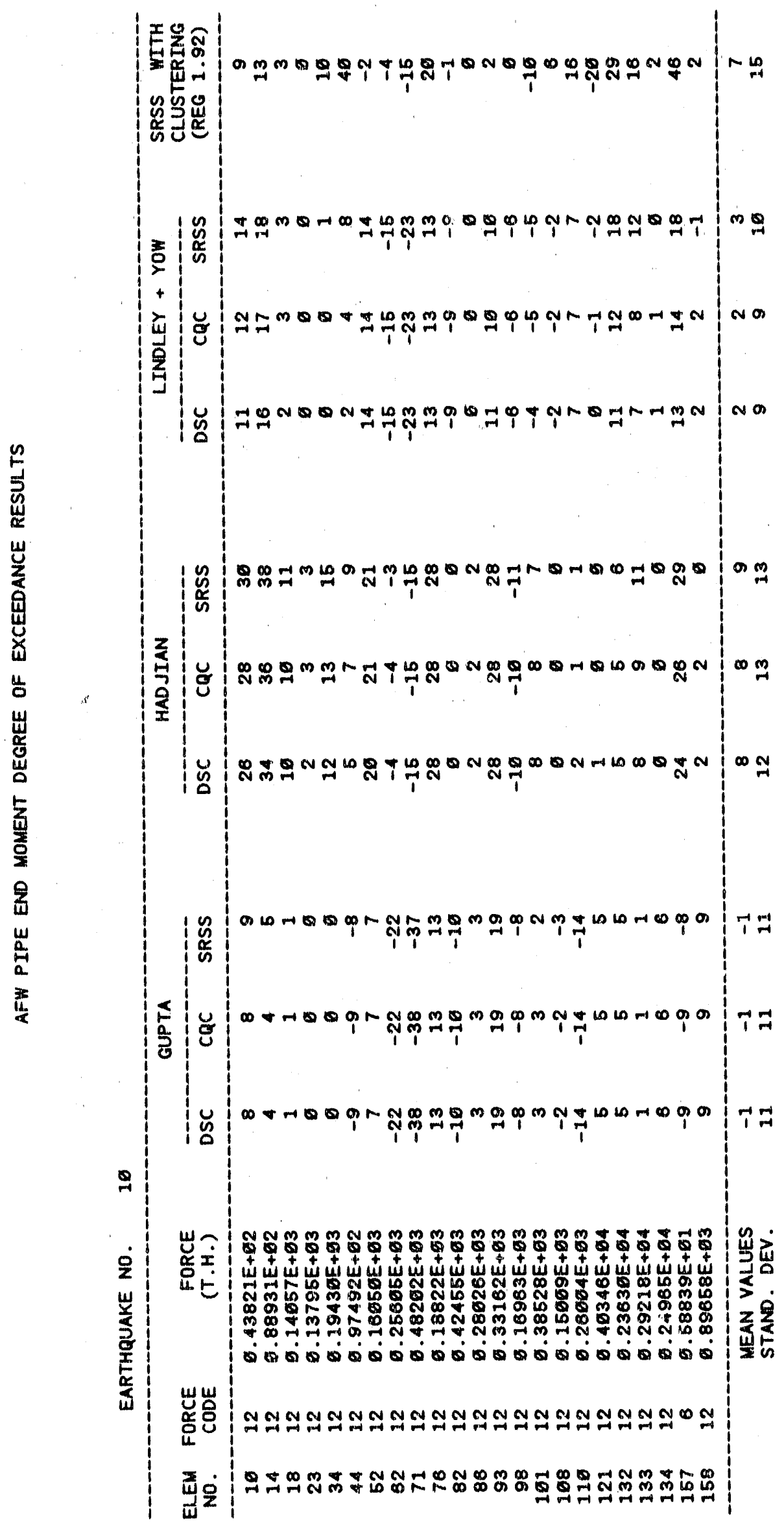

A-87 


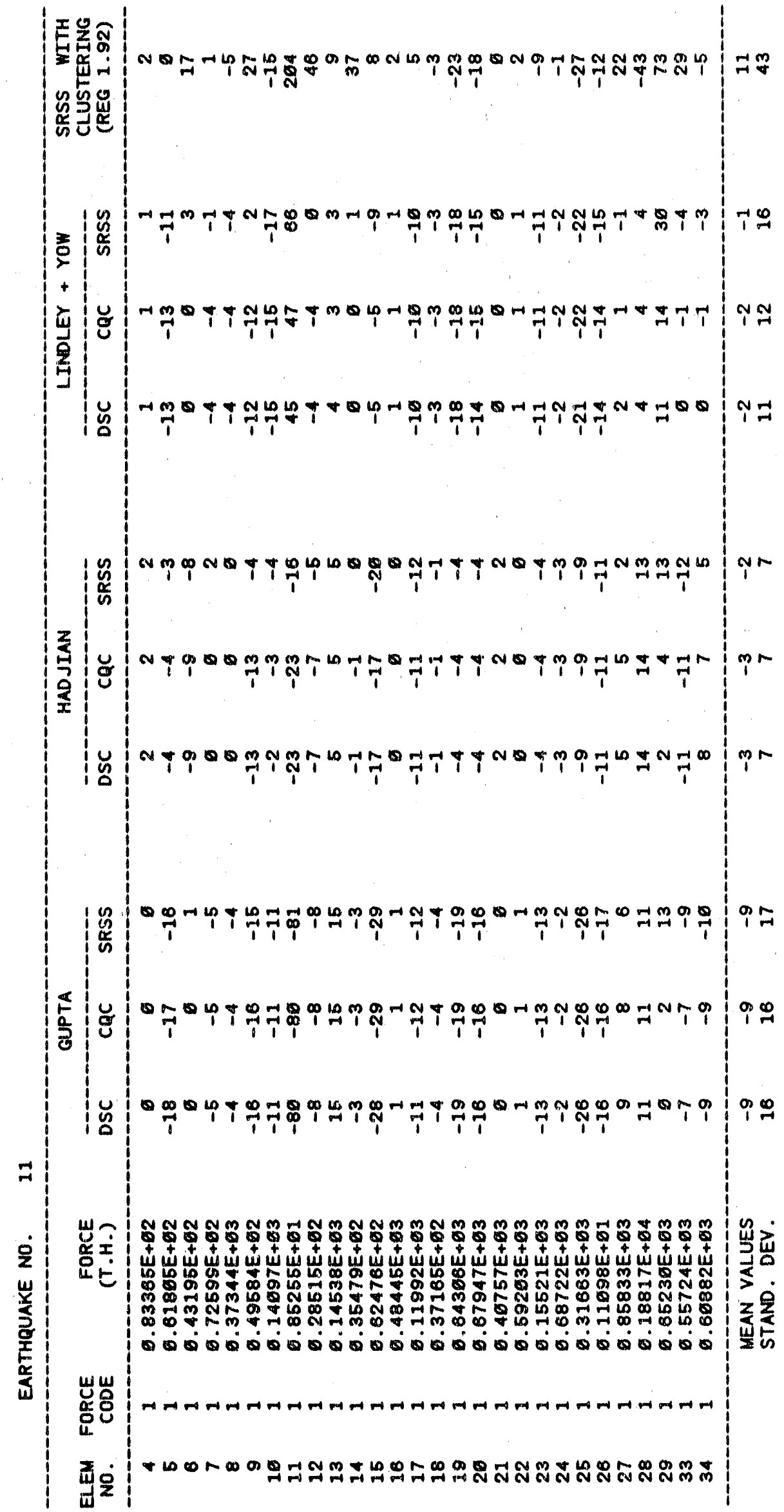




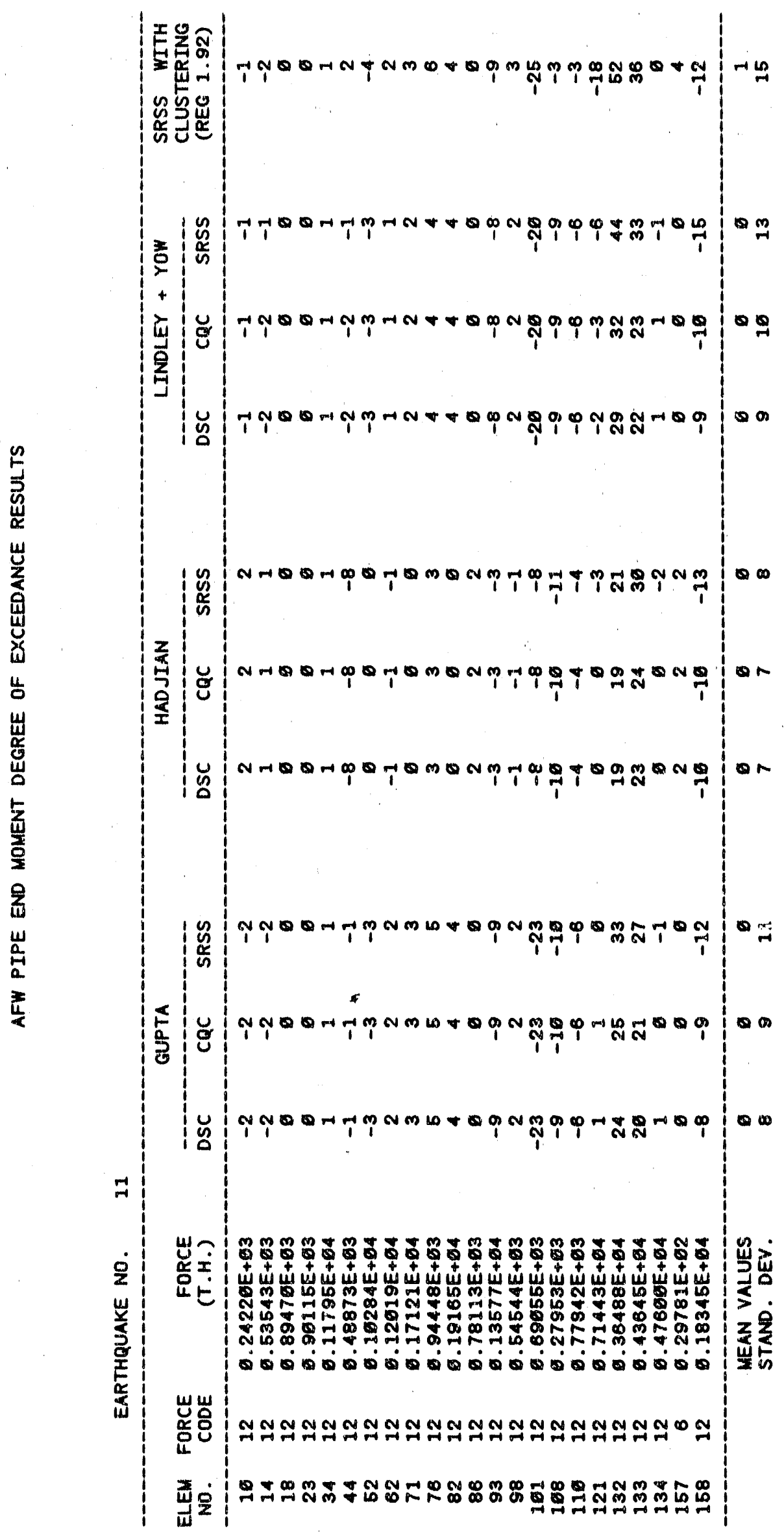

A-89 


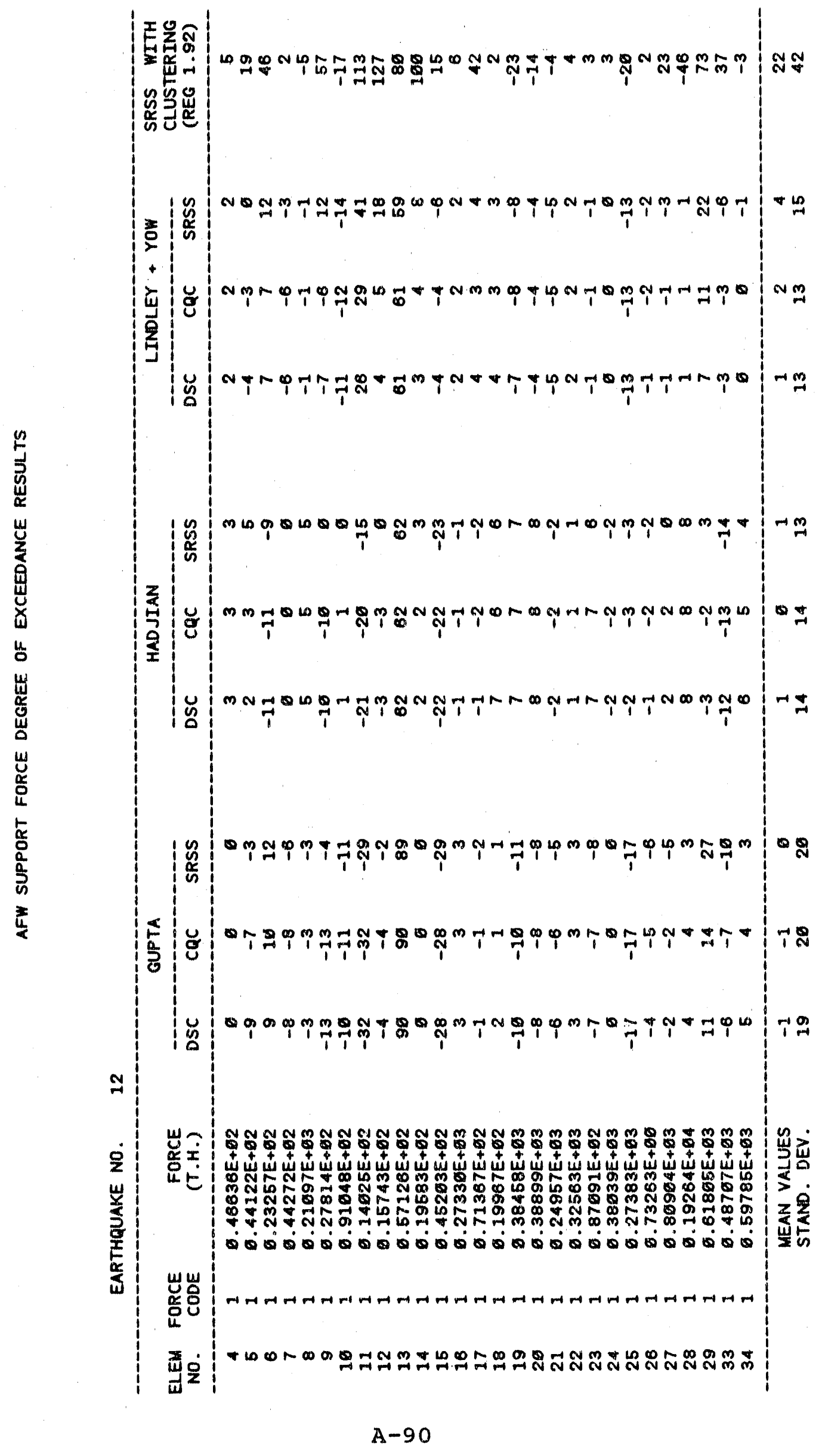




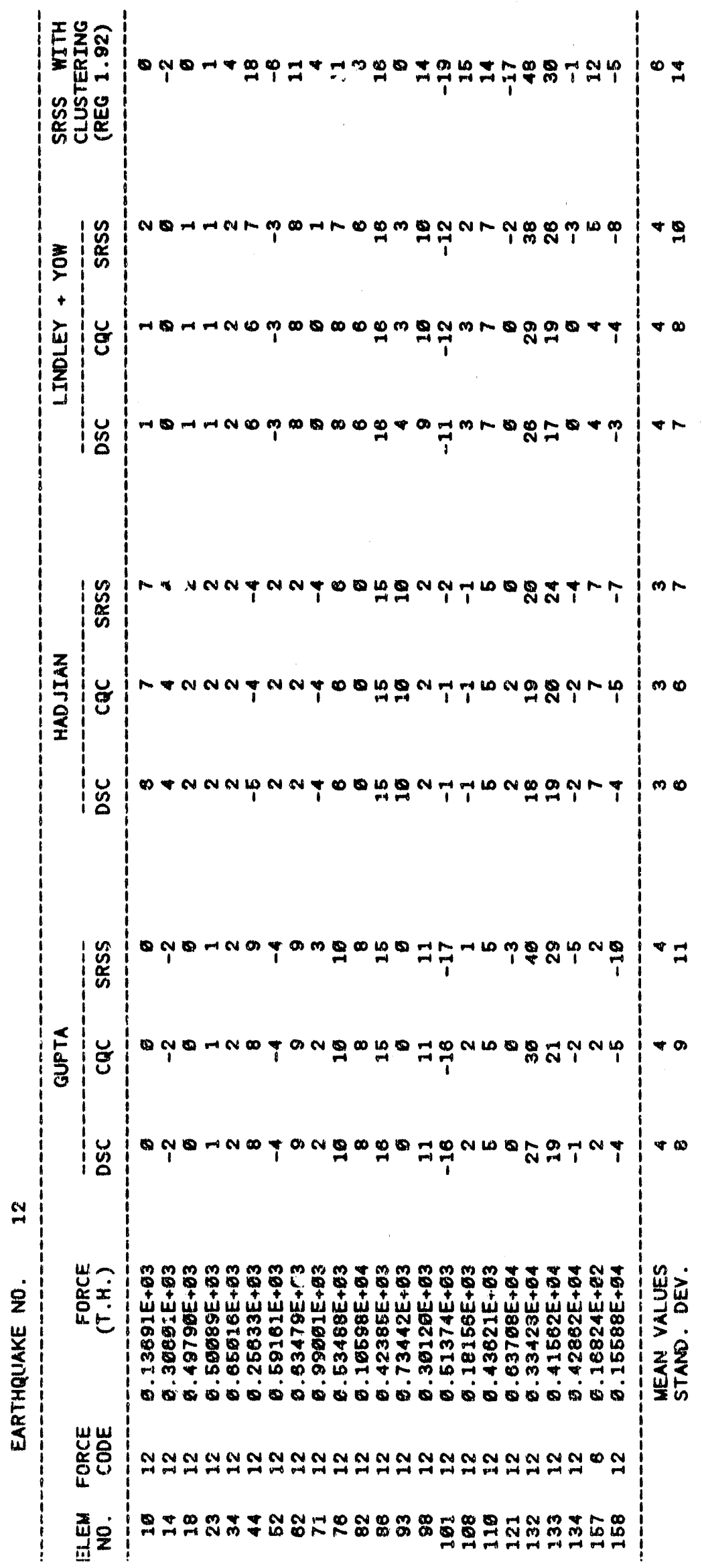




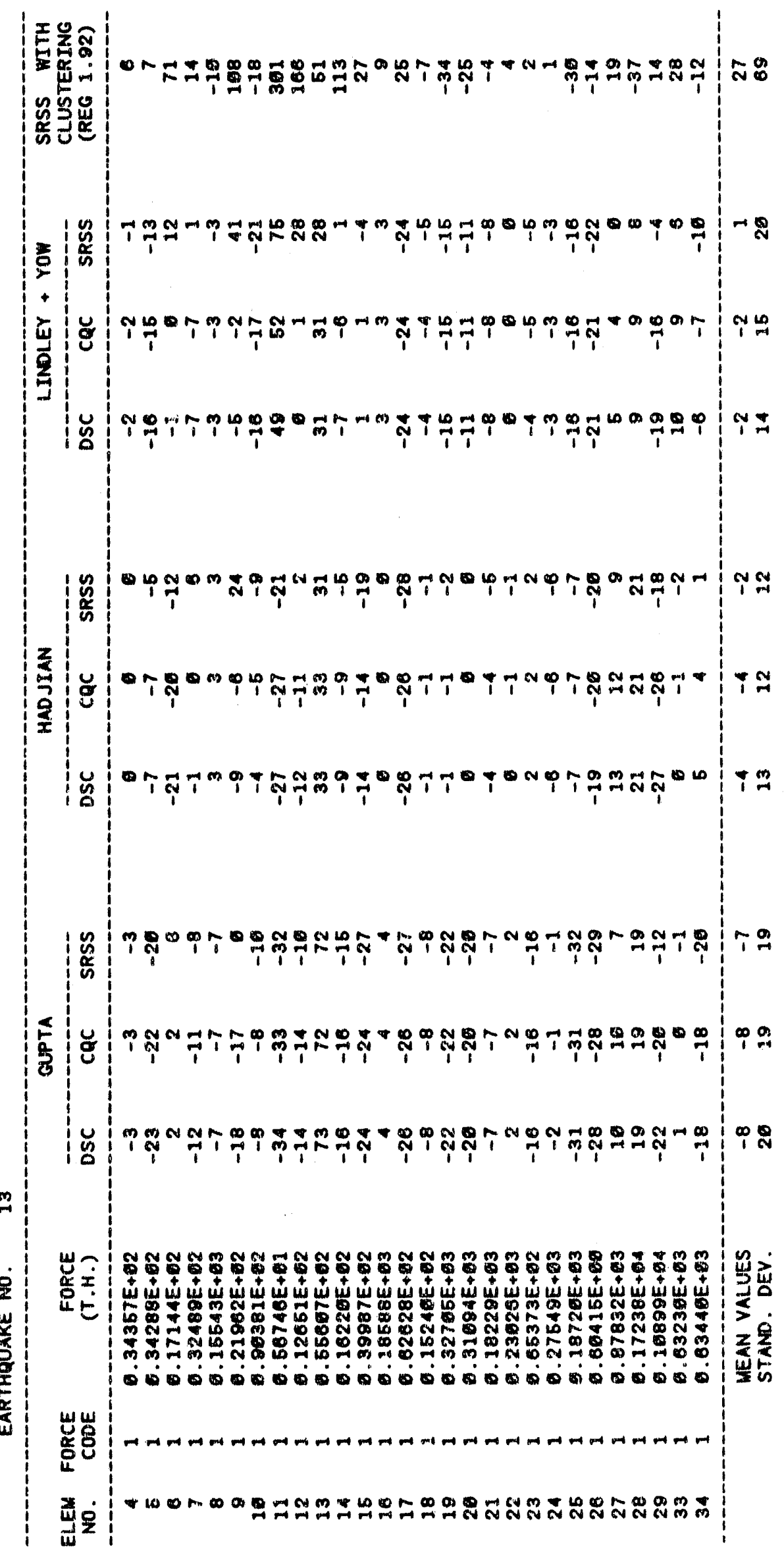




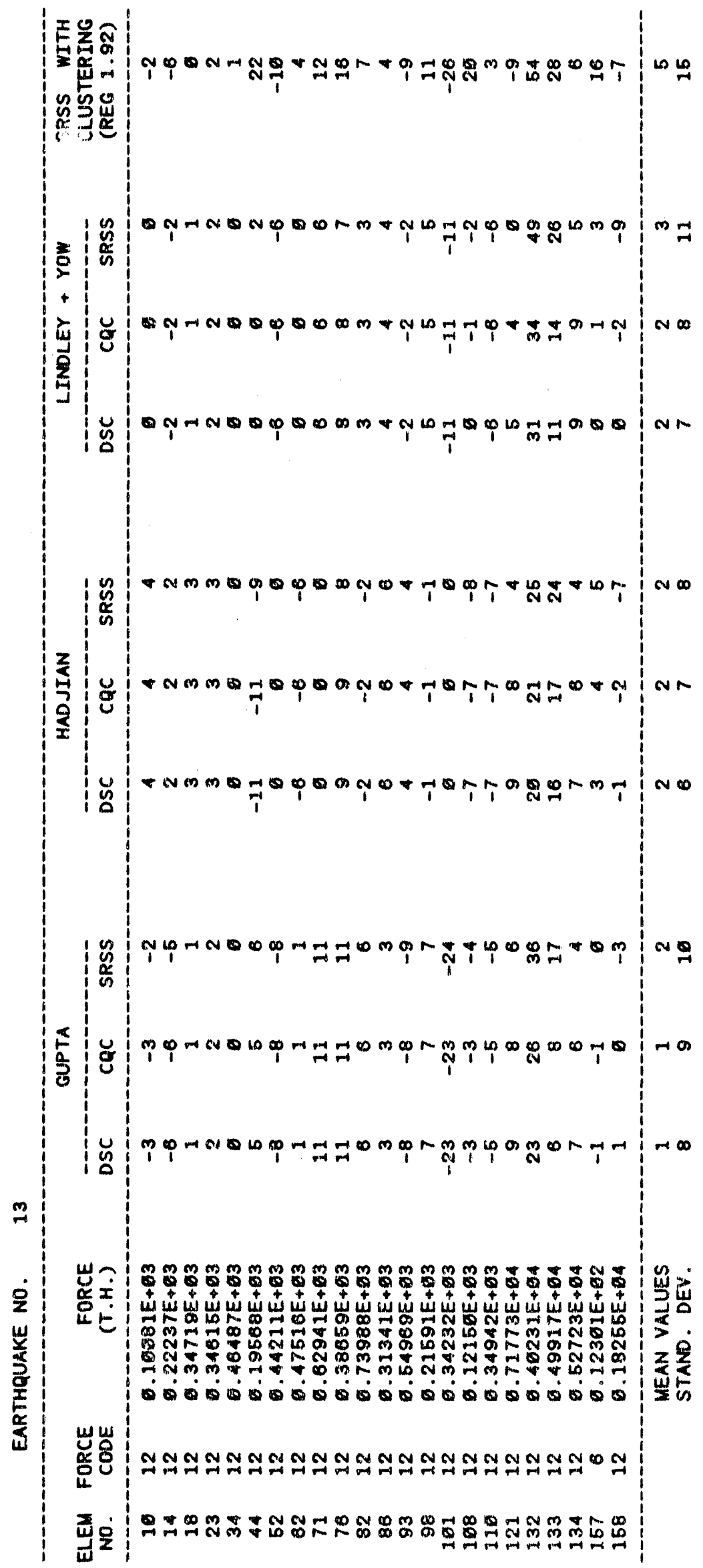

A-93 


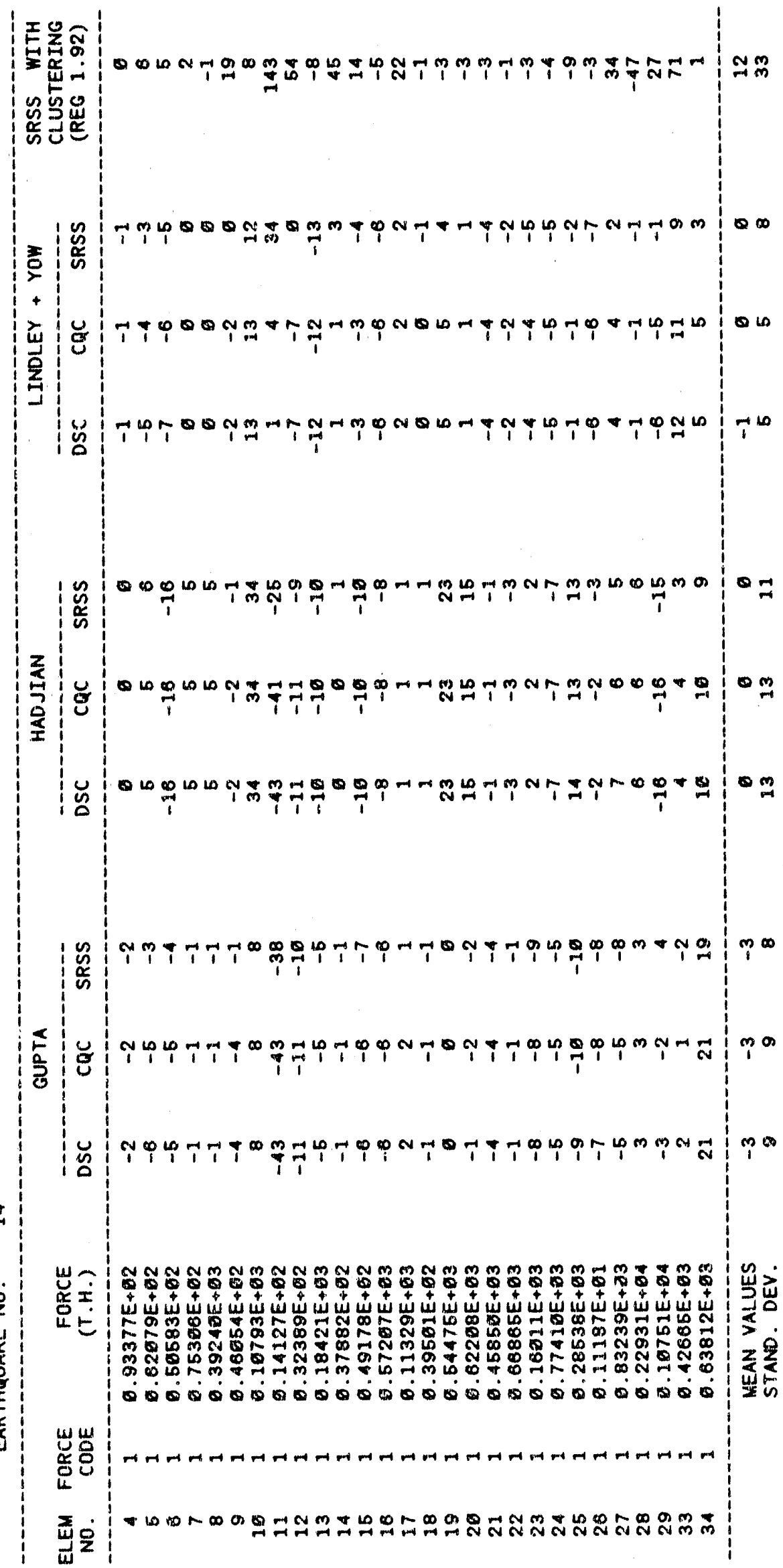

$$
\text { A-94 }
$$




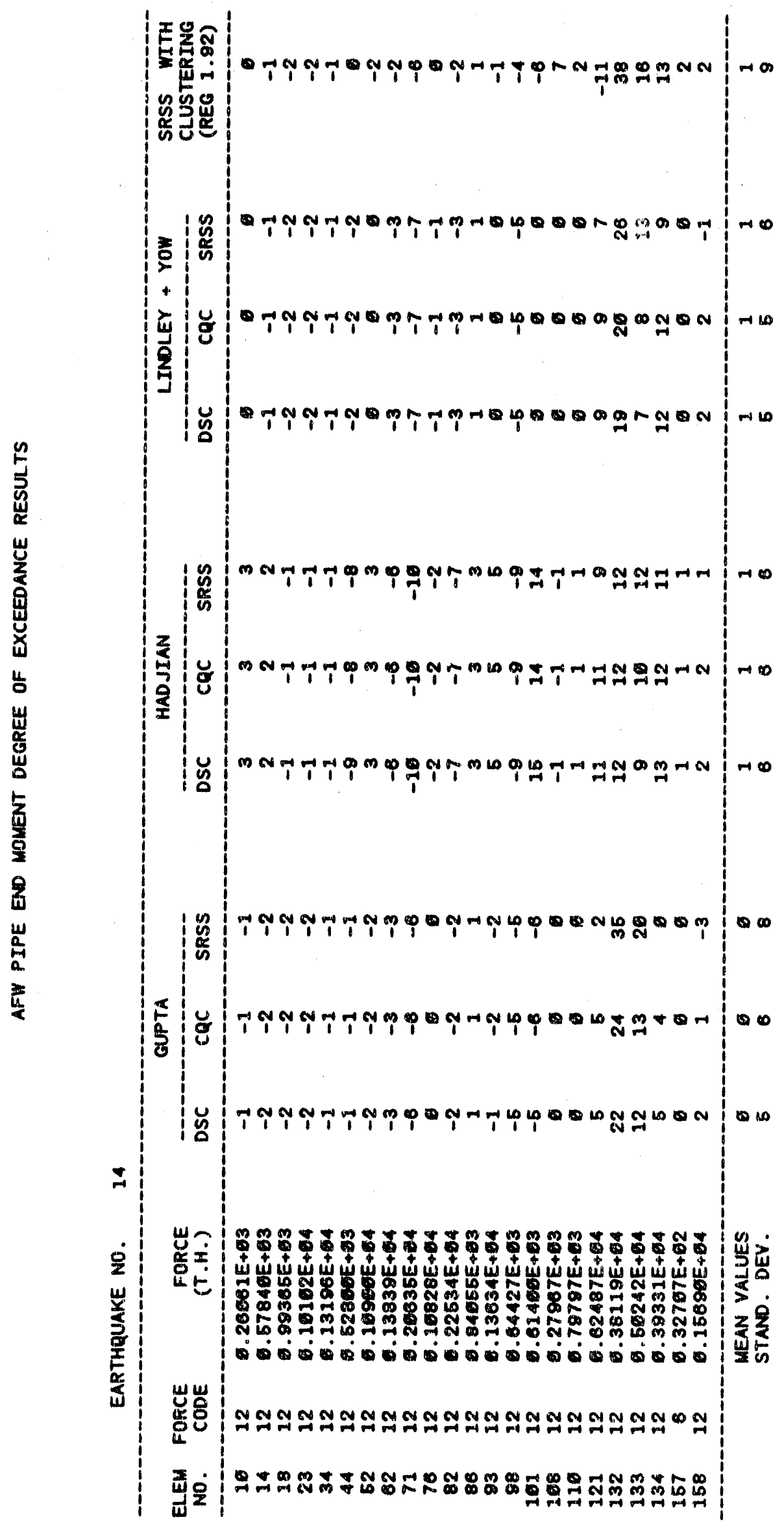




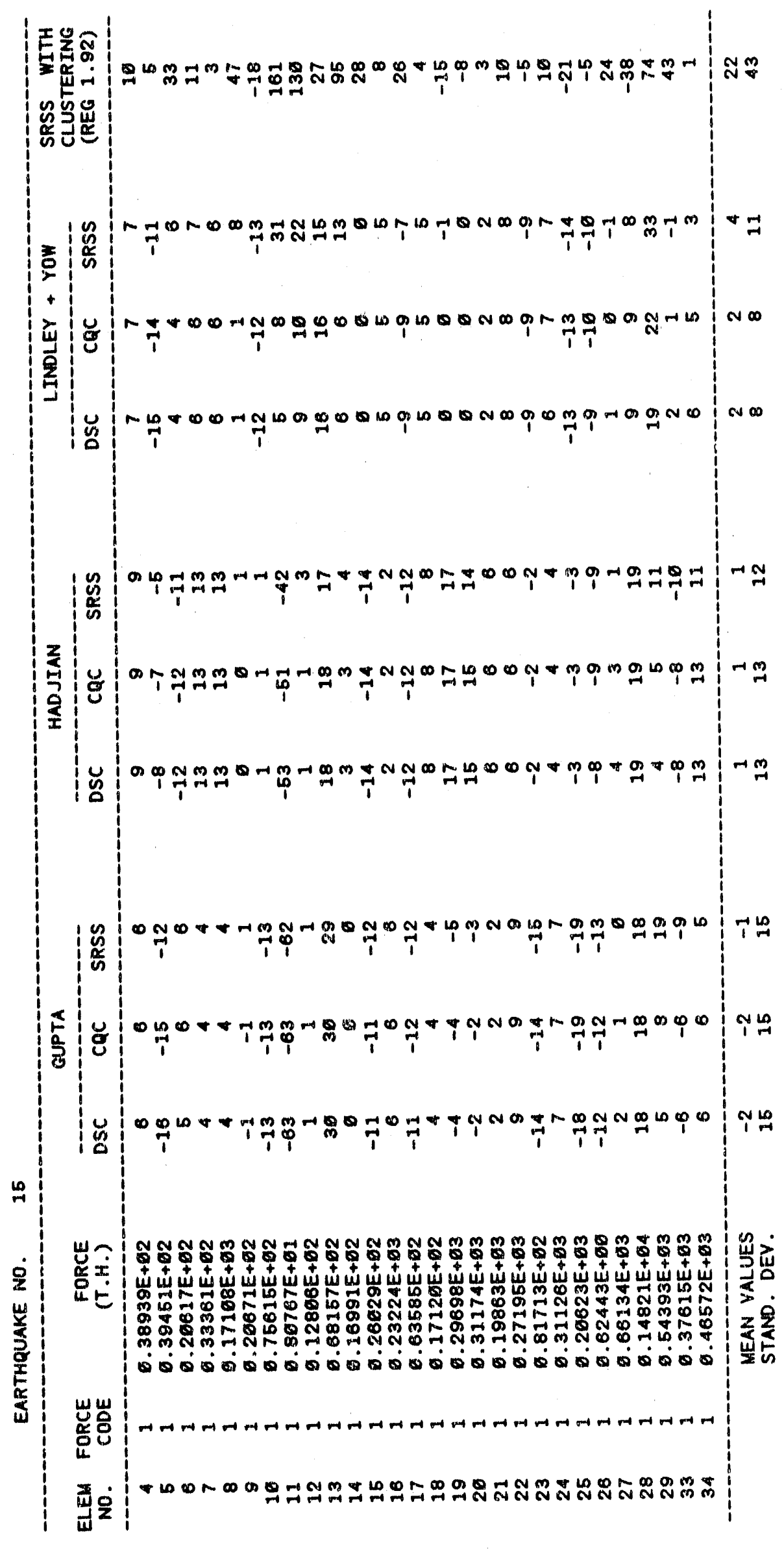




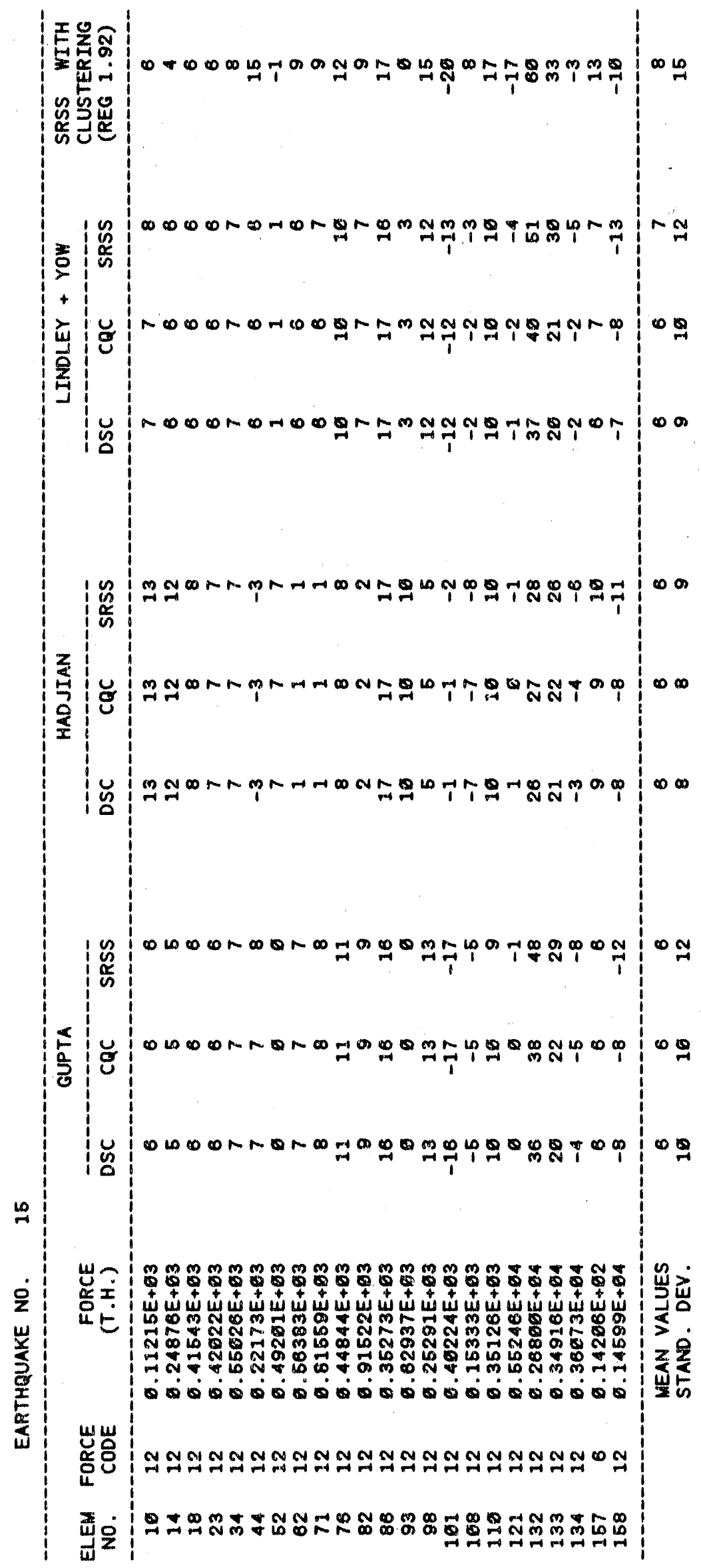




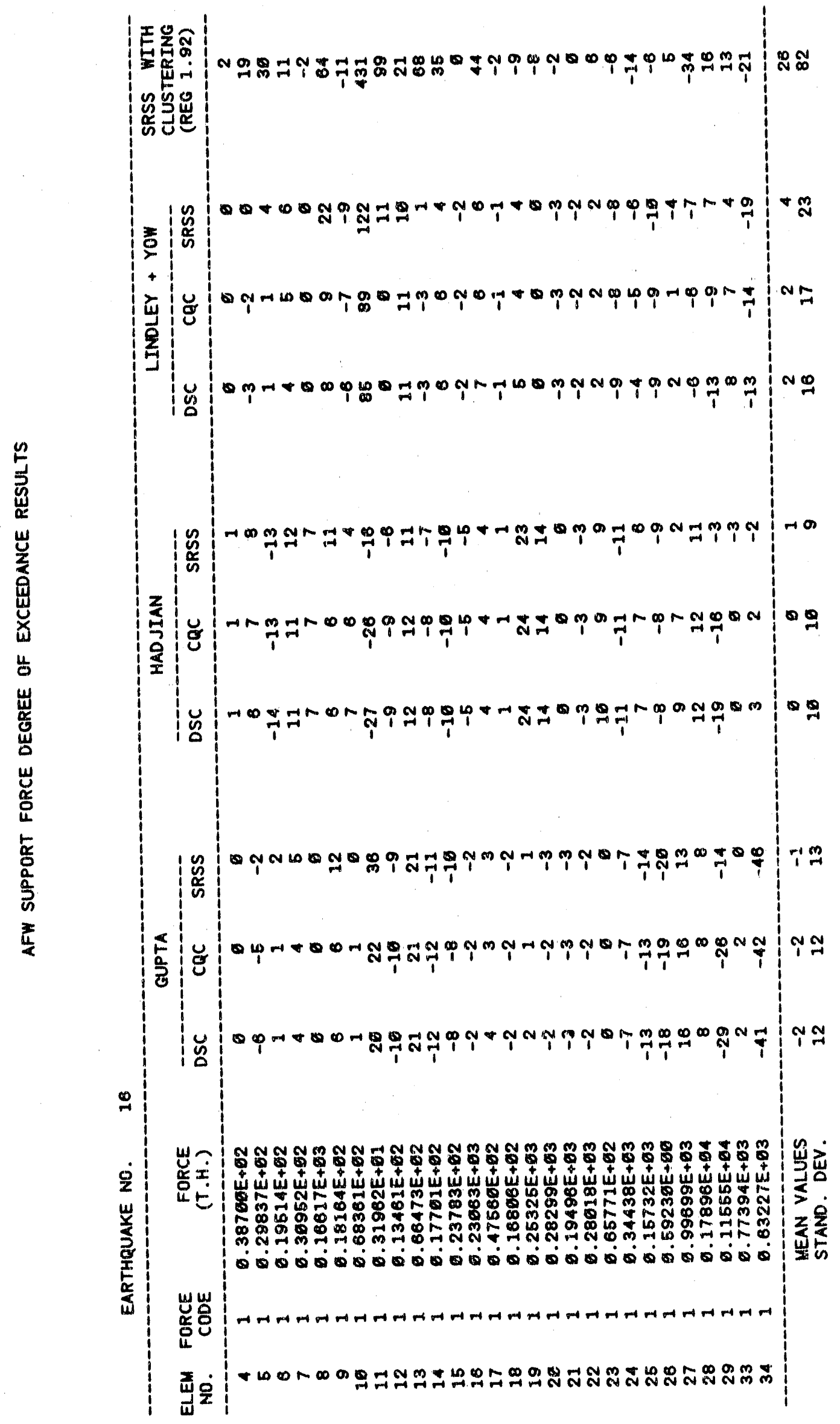




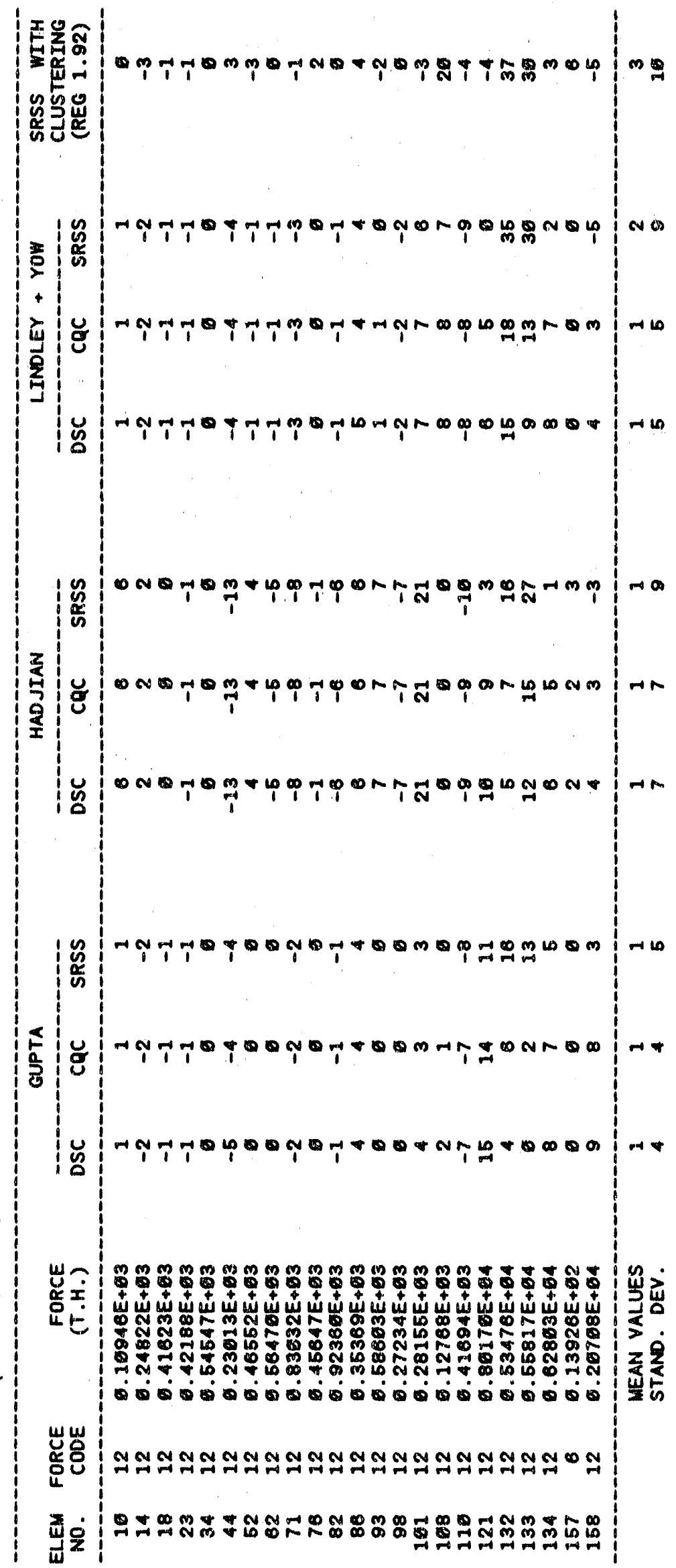

A-99 


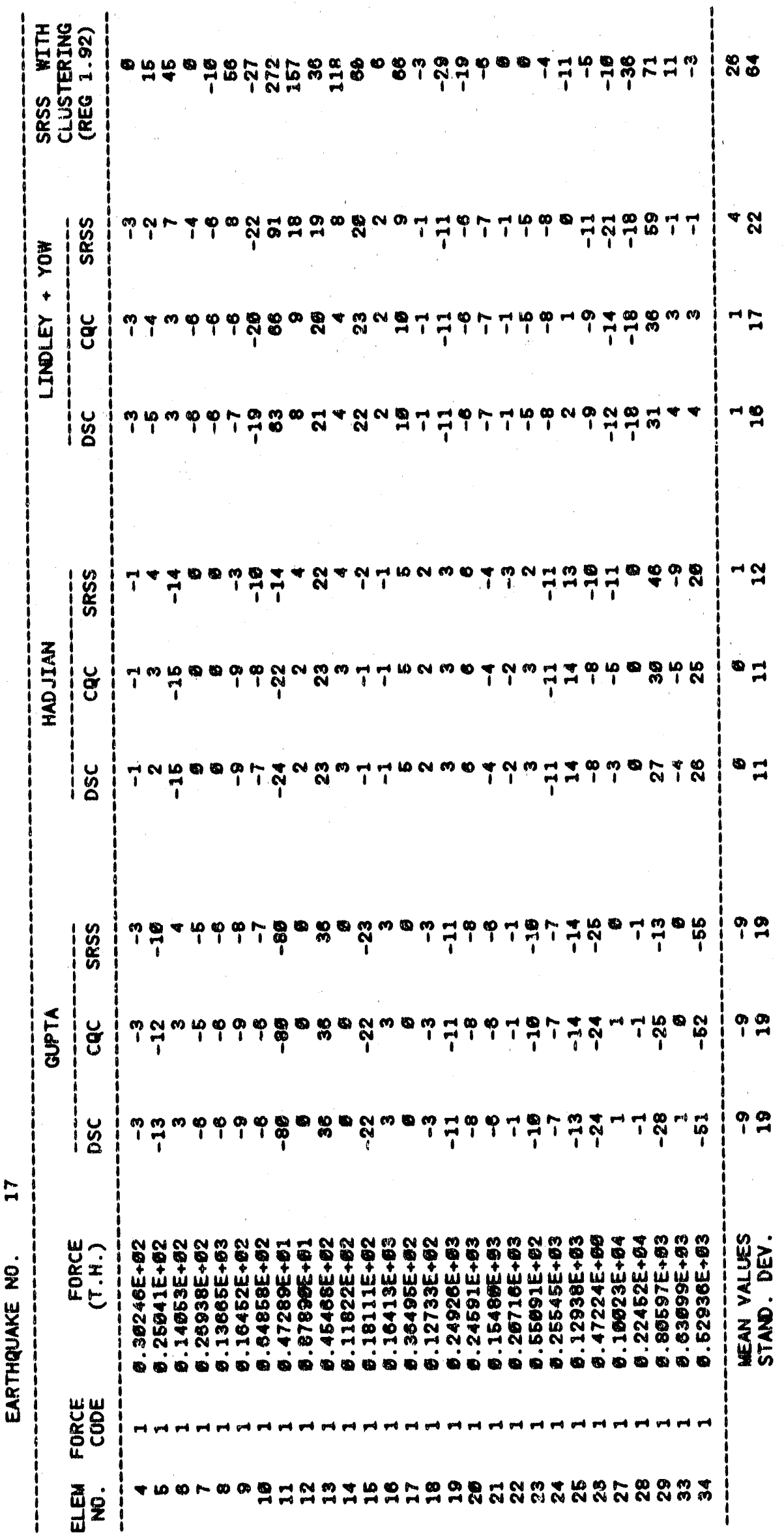

$$
A-100
$$




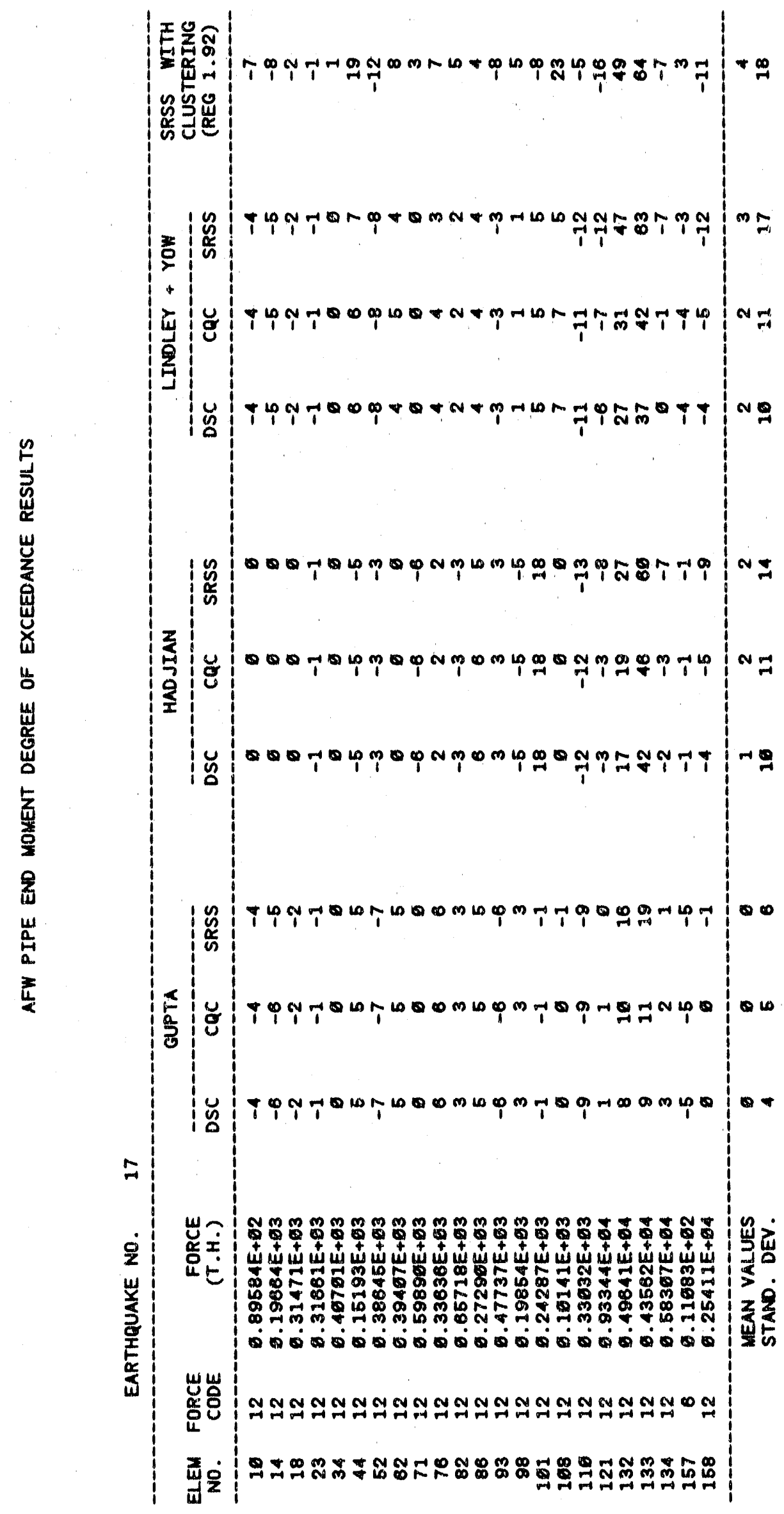




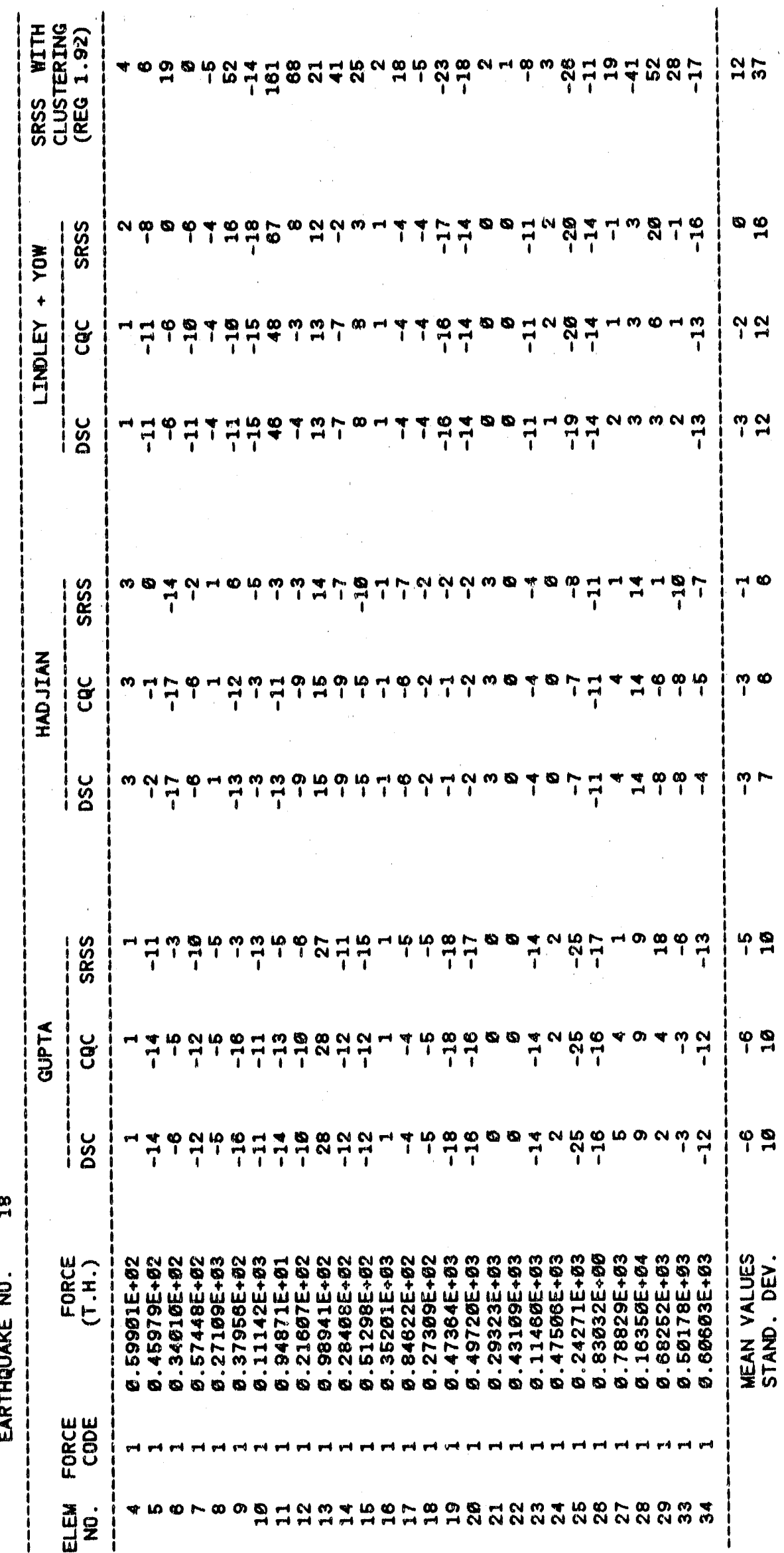

$$
\text { A-102 }
$$




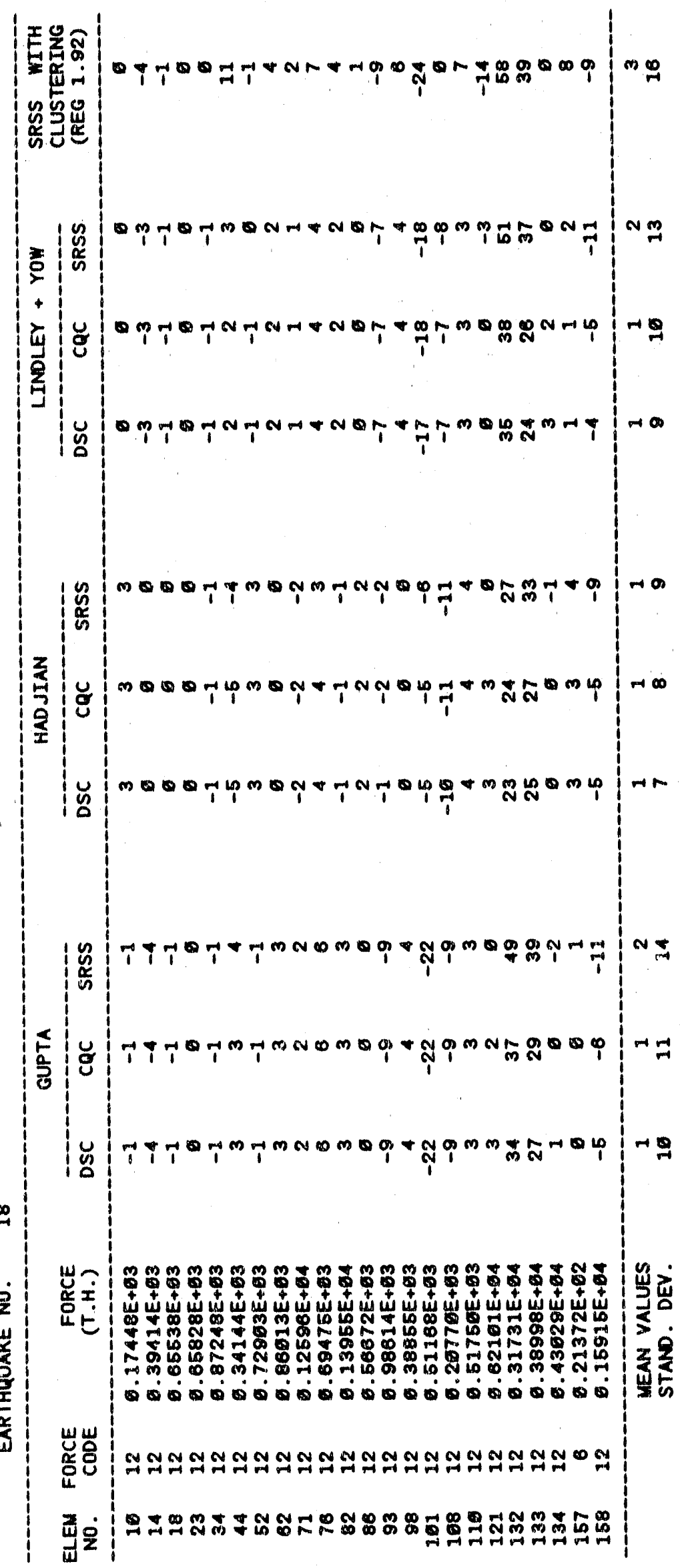

$$
A-103
$$




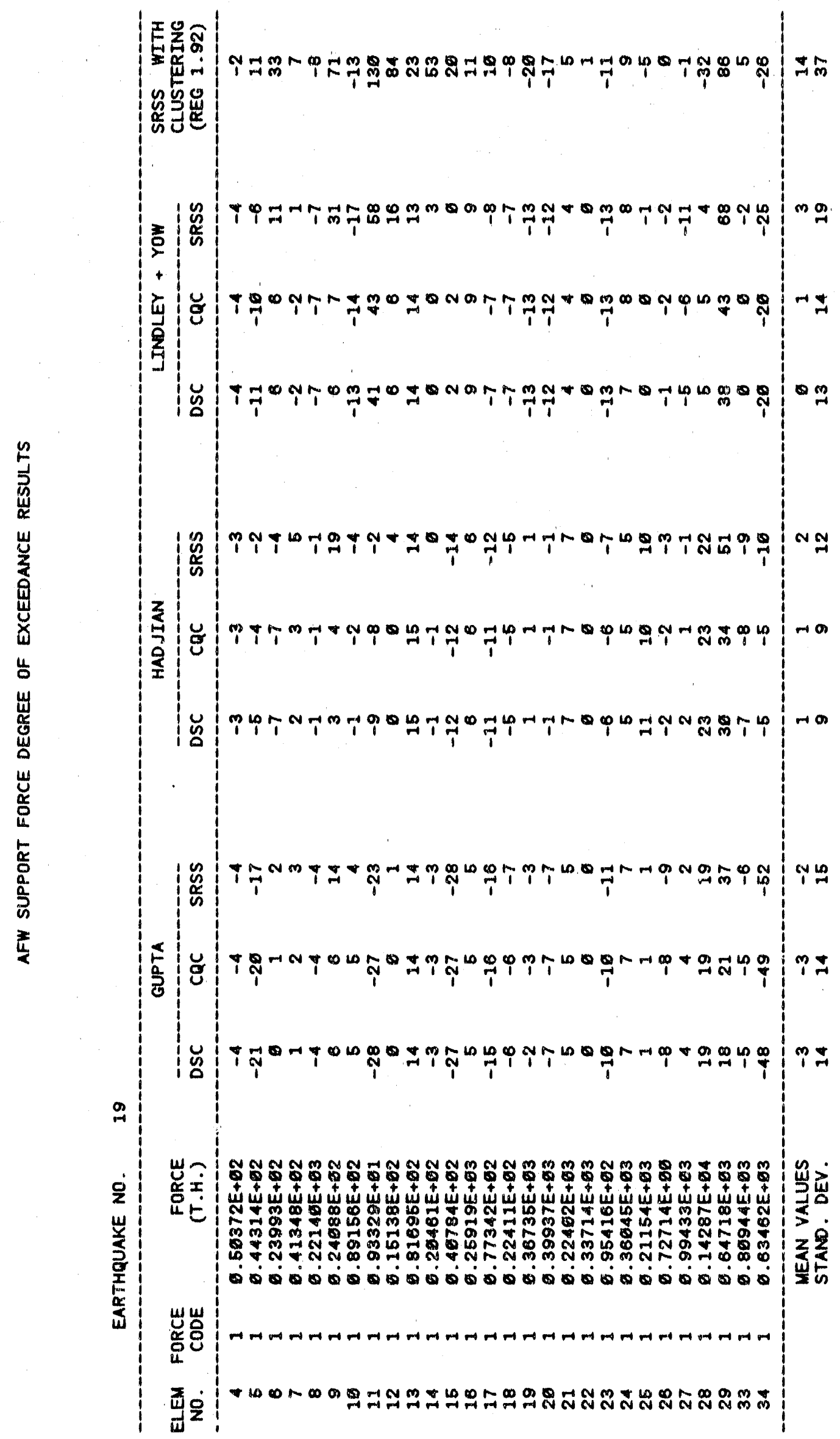




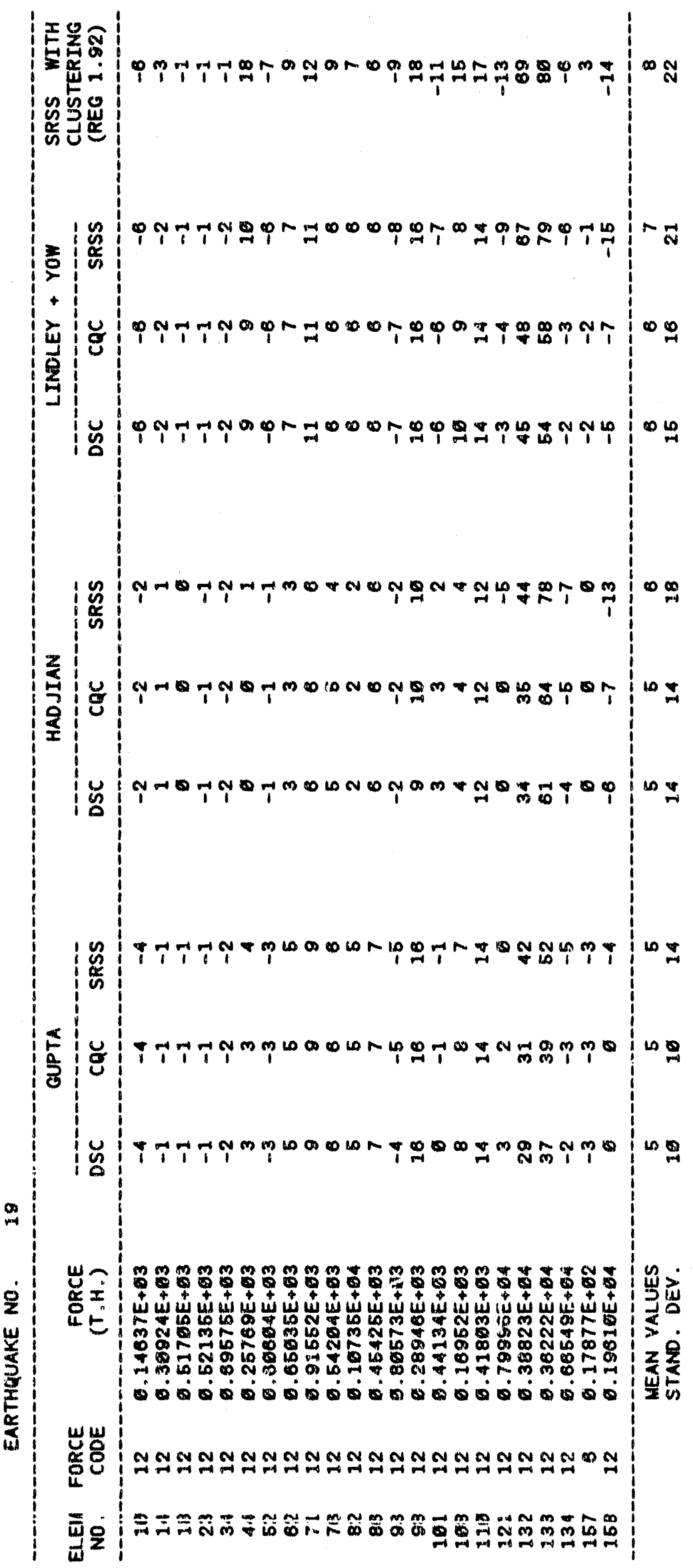




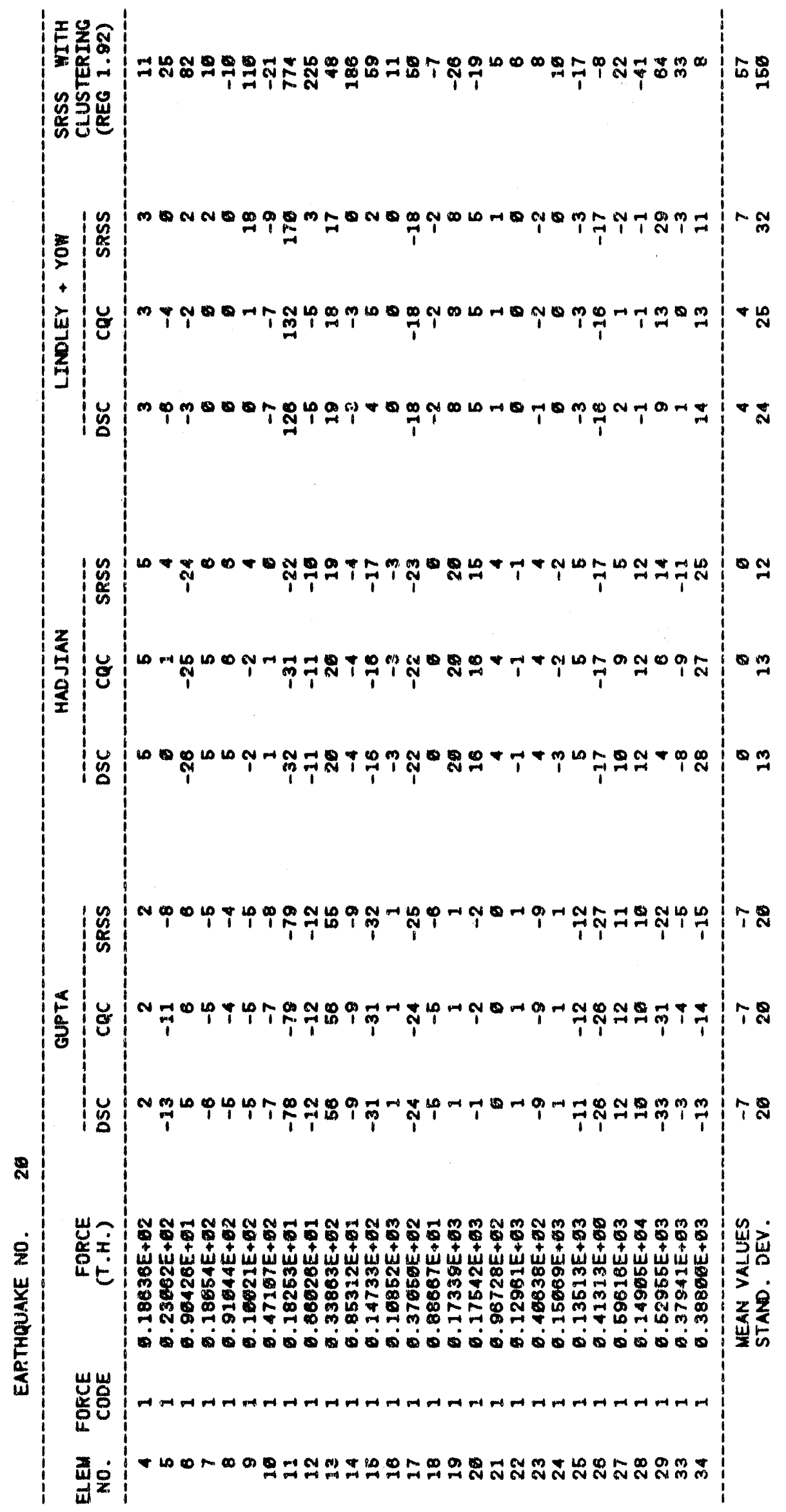




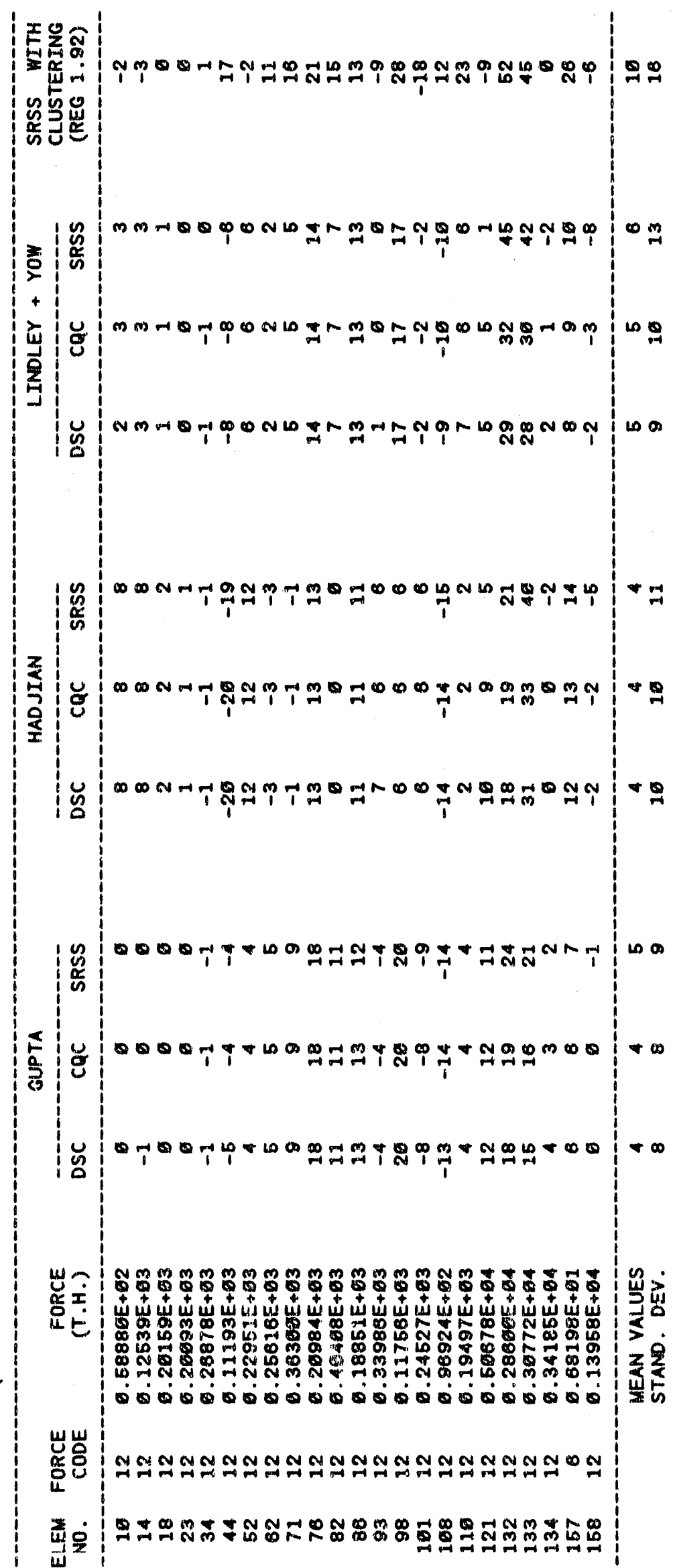




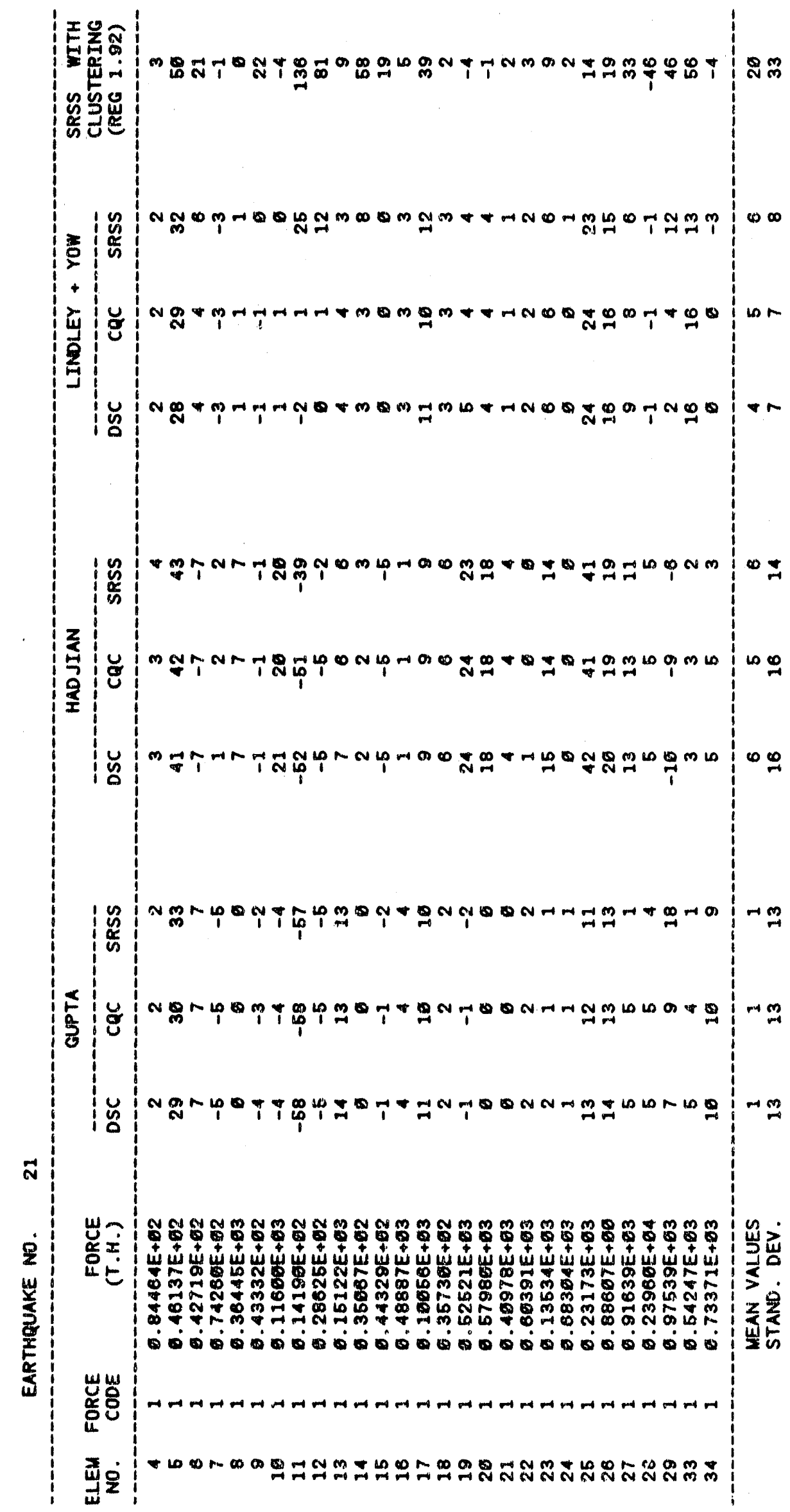




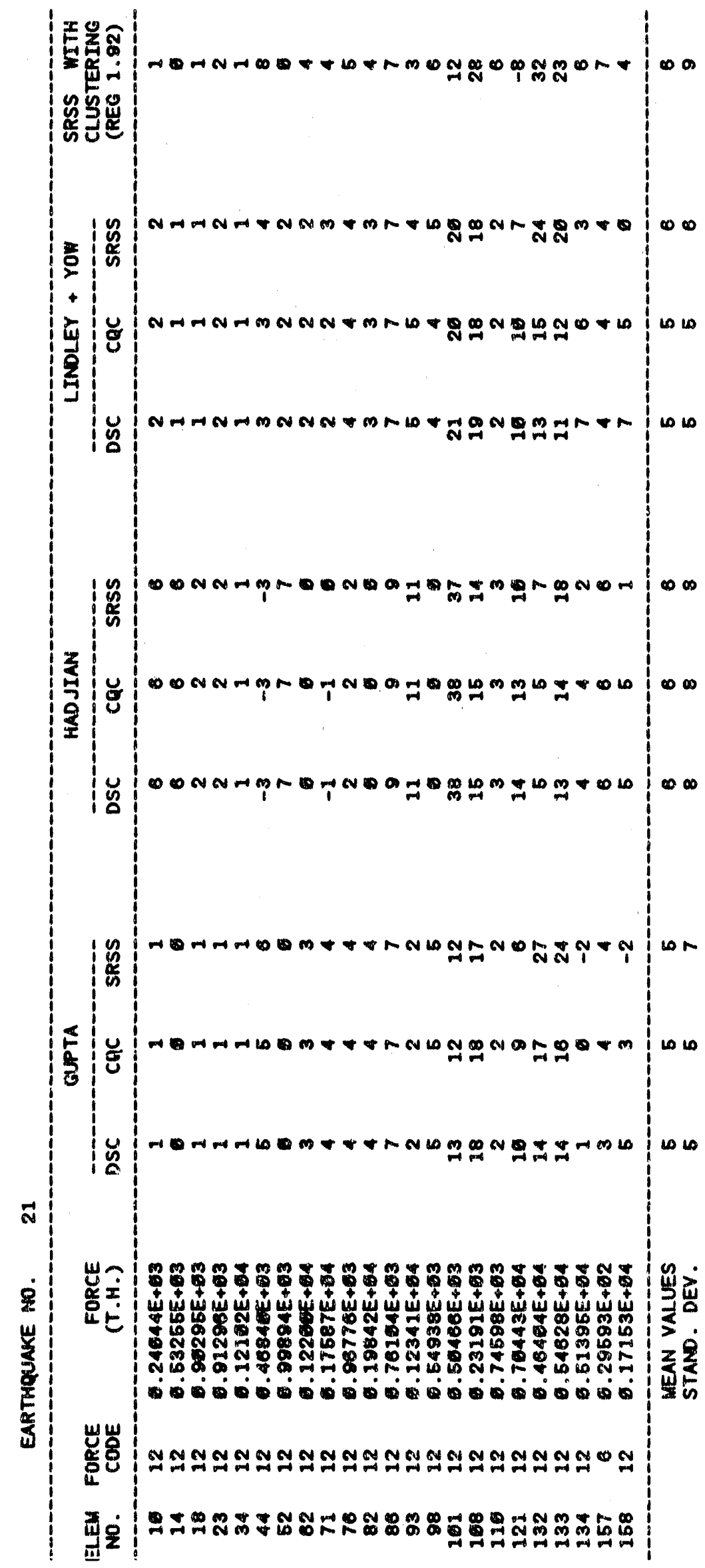




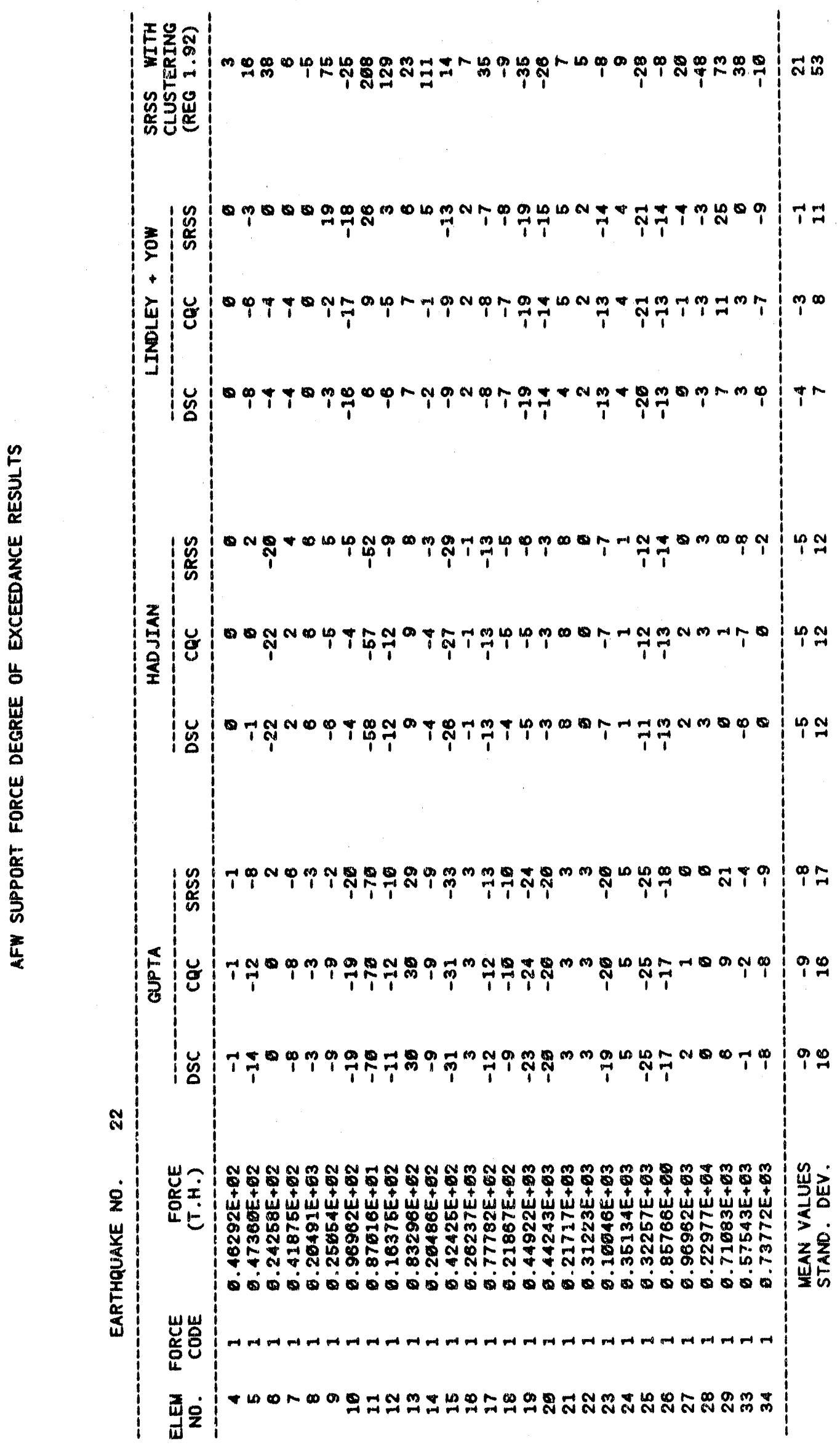

A-110 


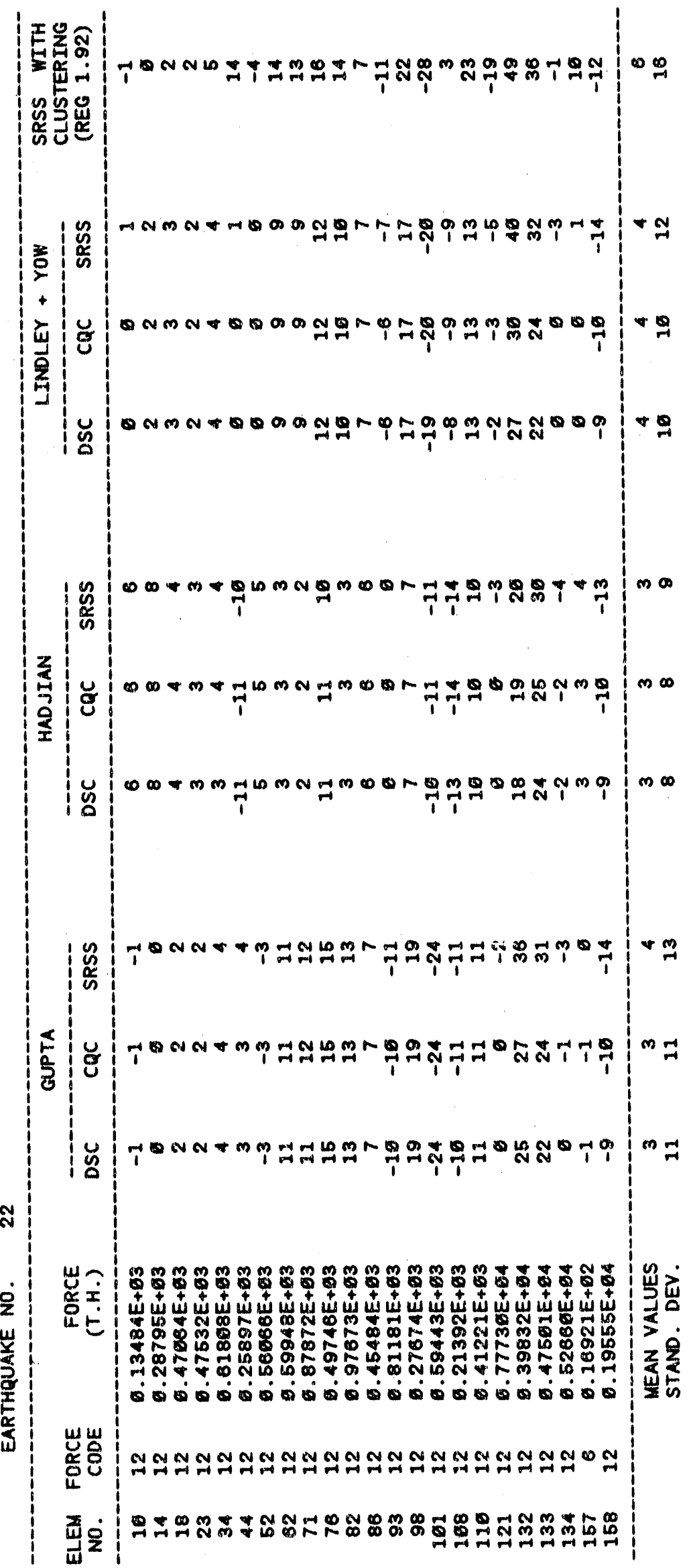




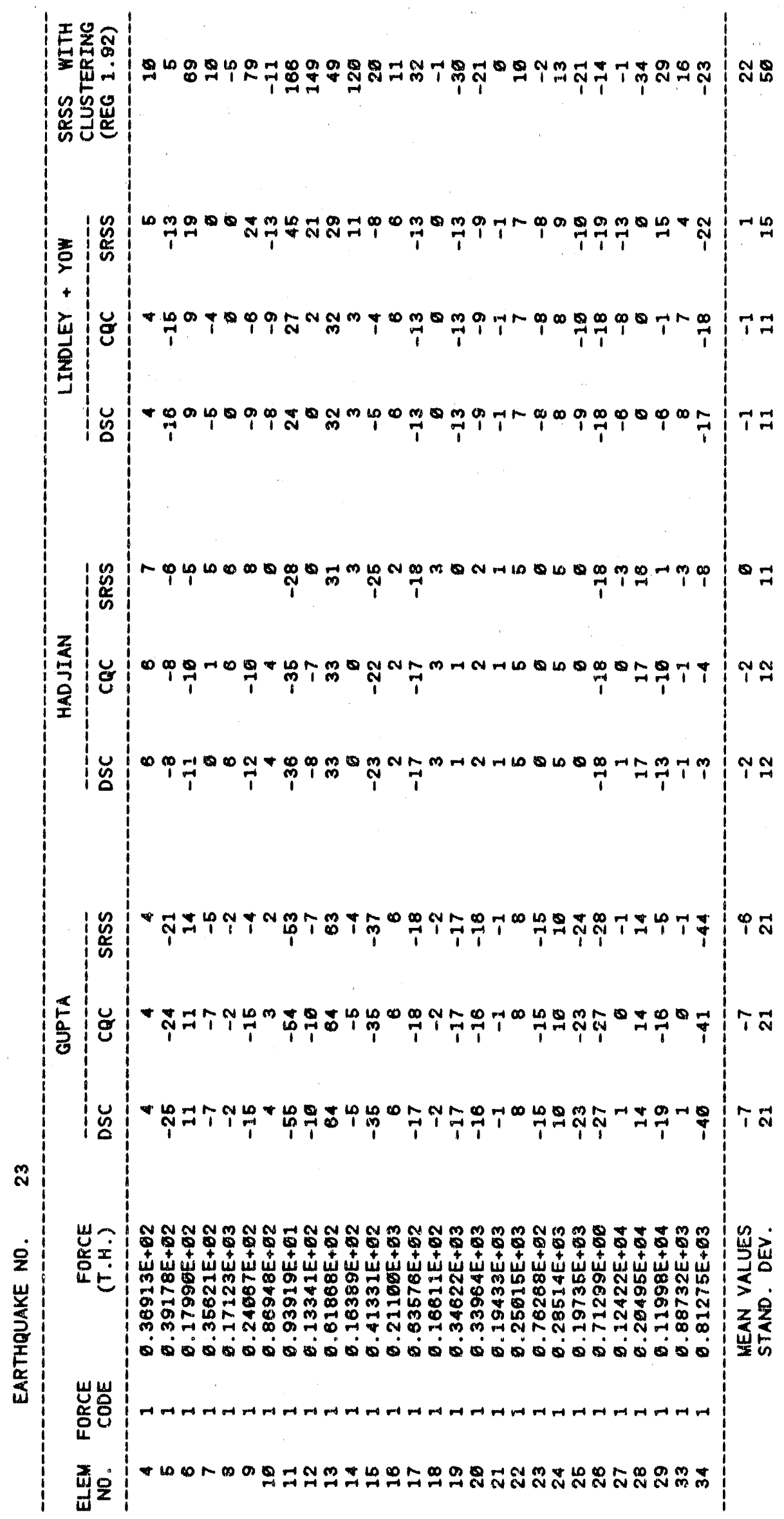




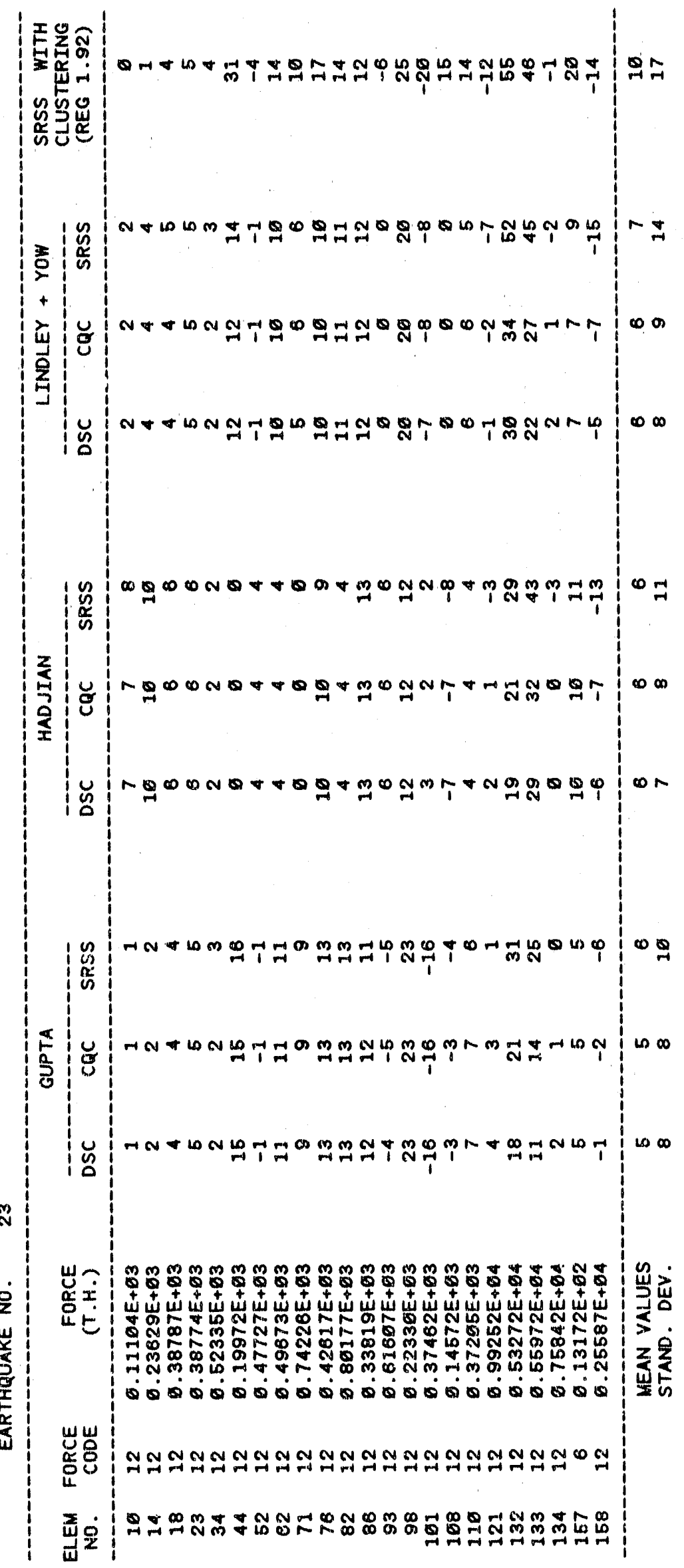




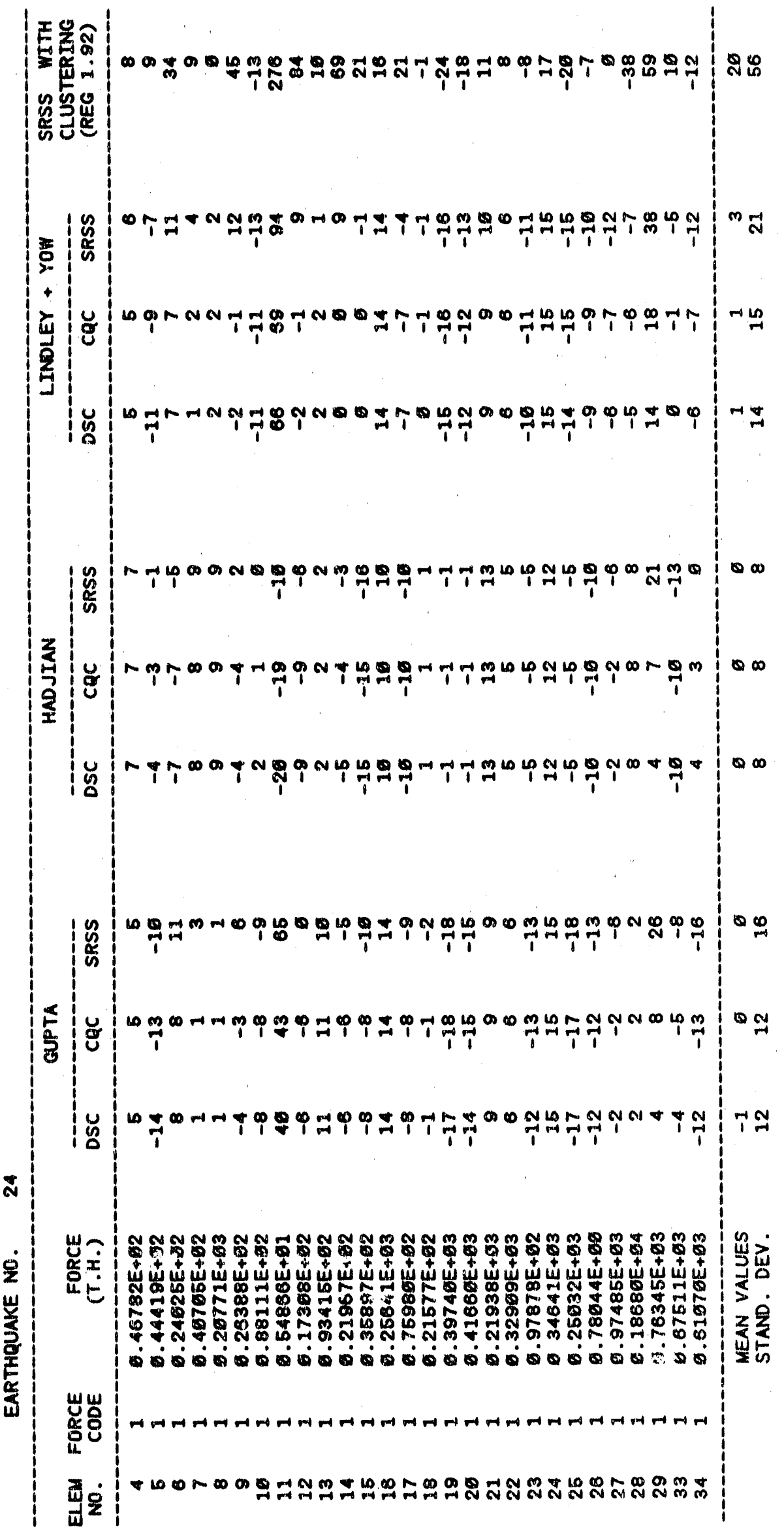




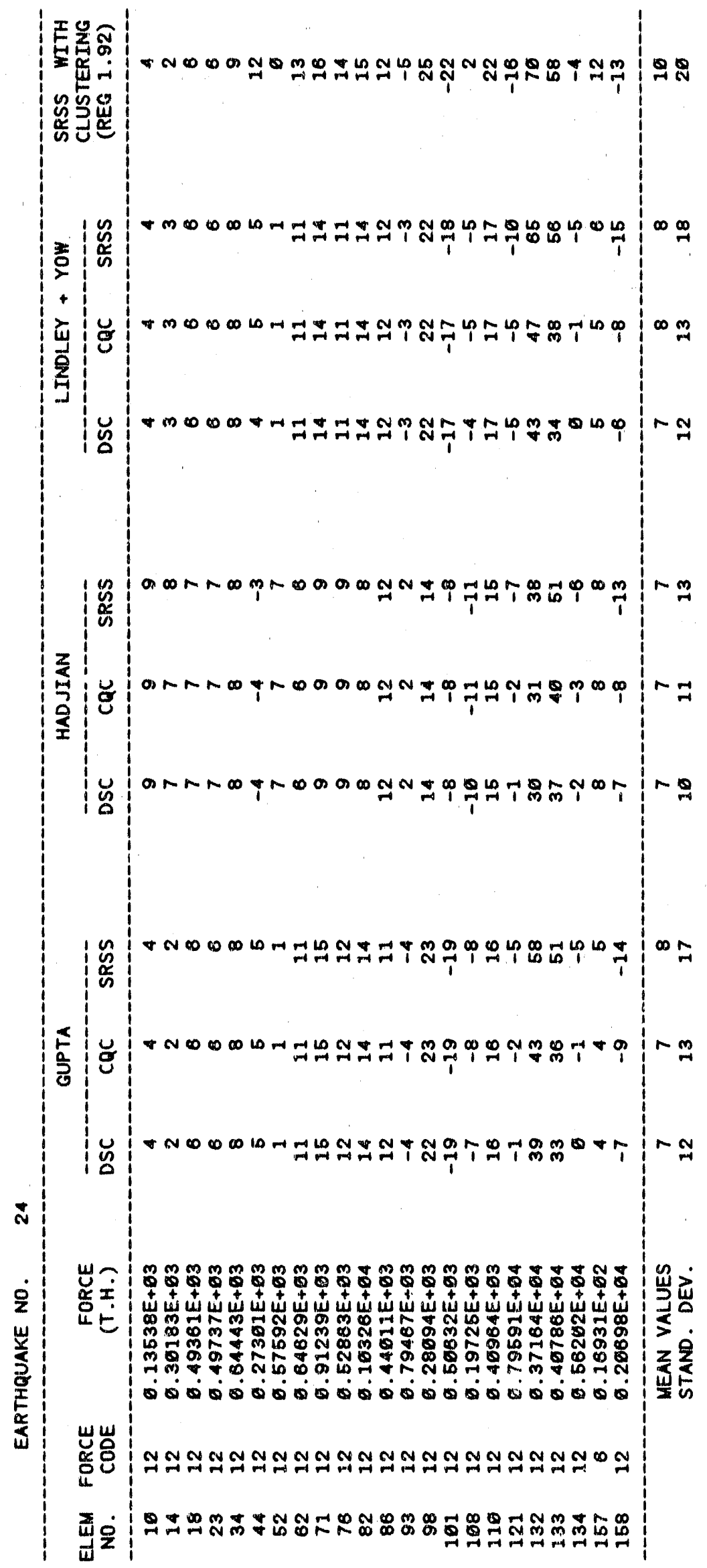




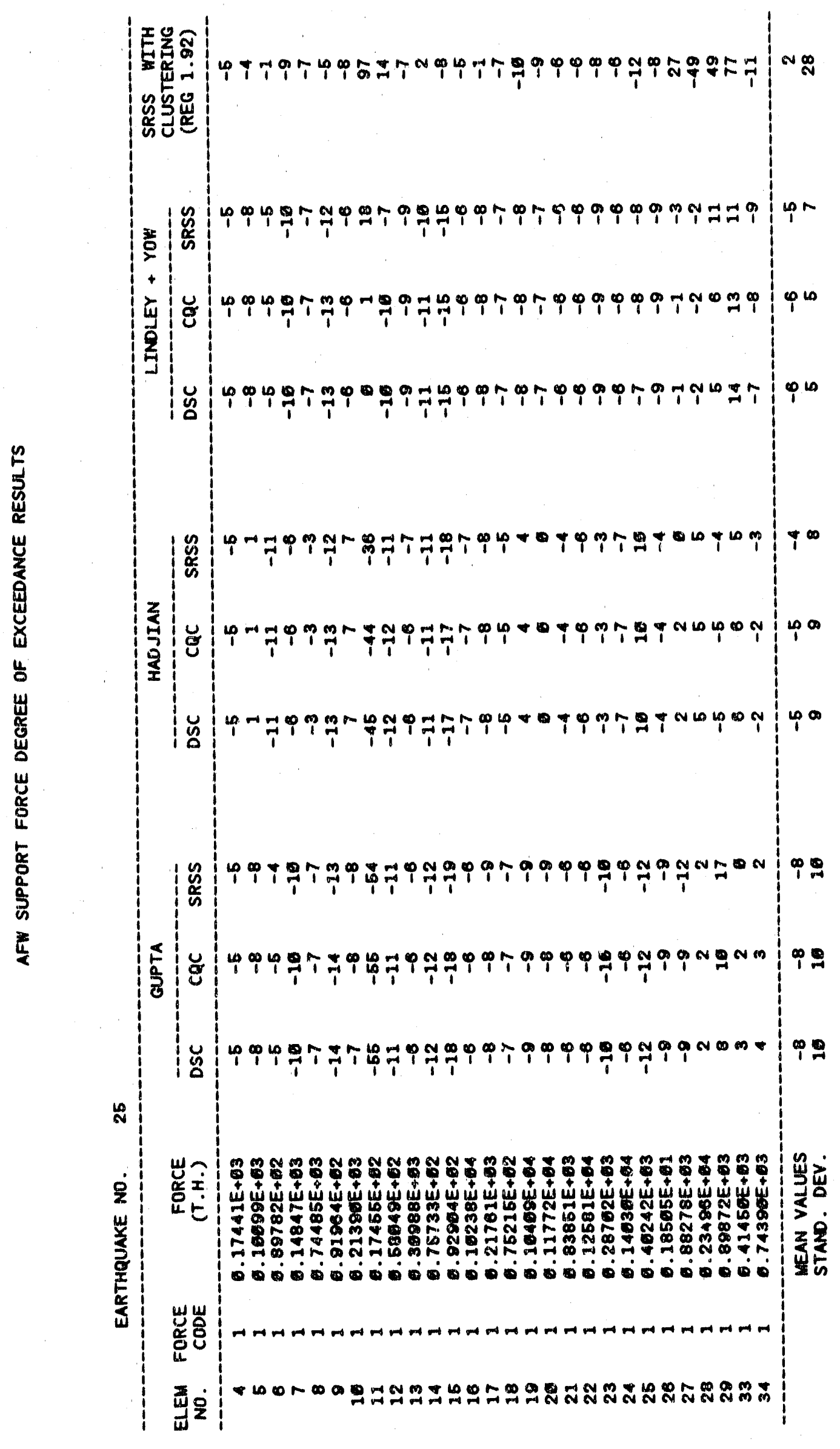




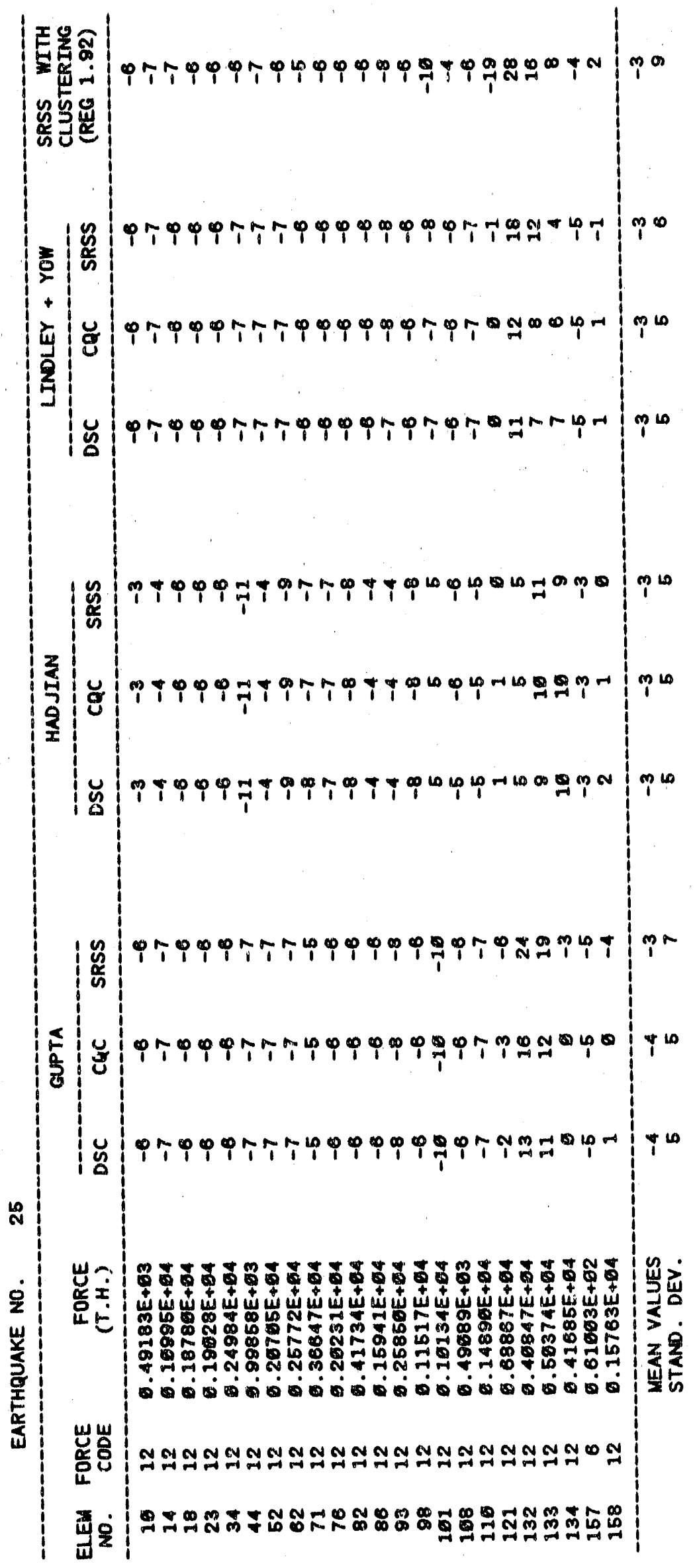




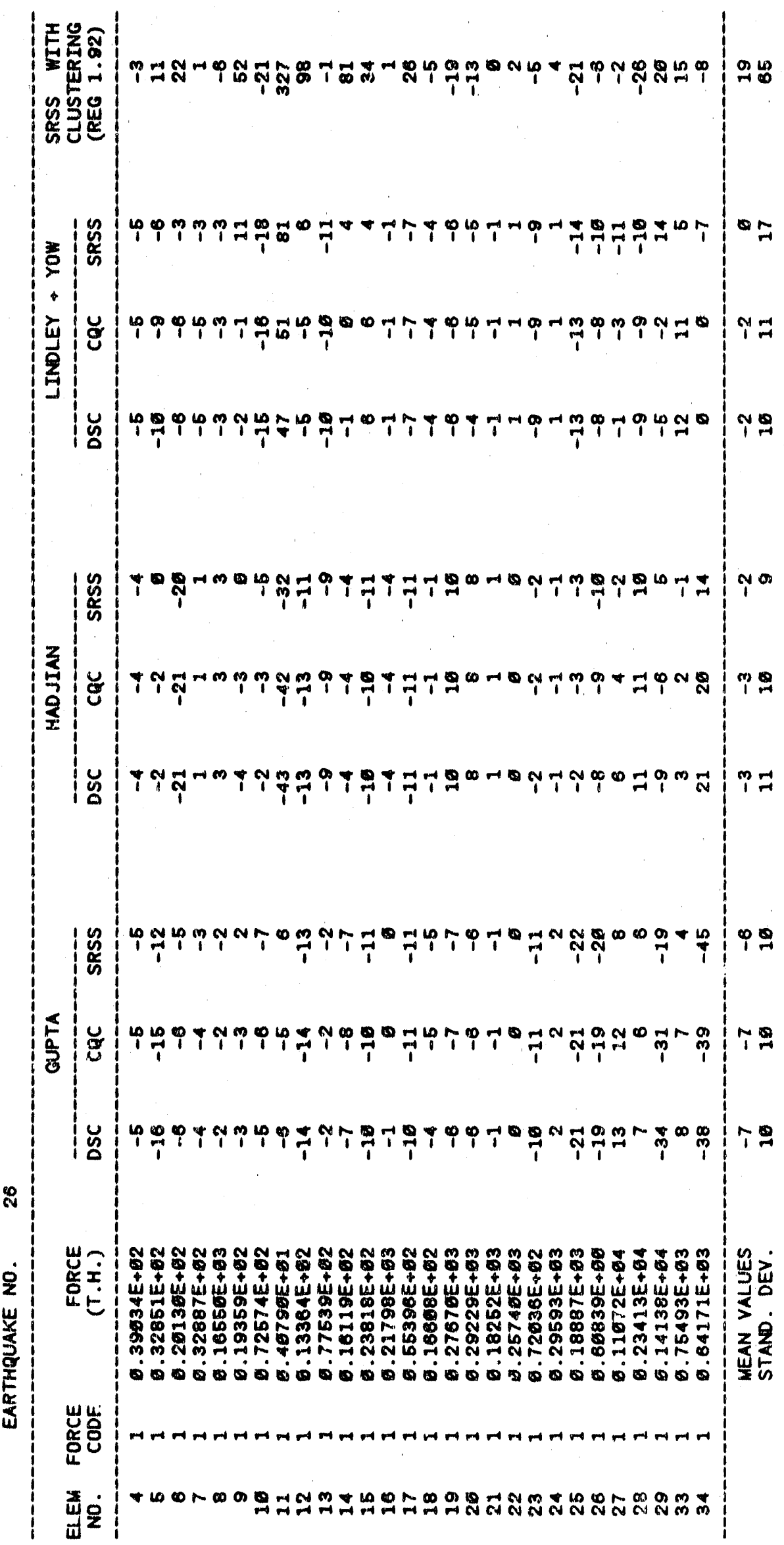




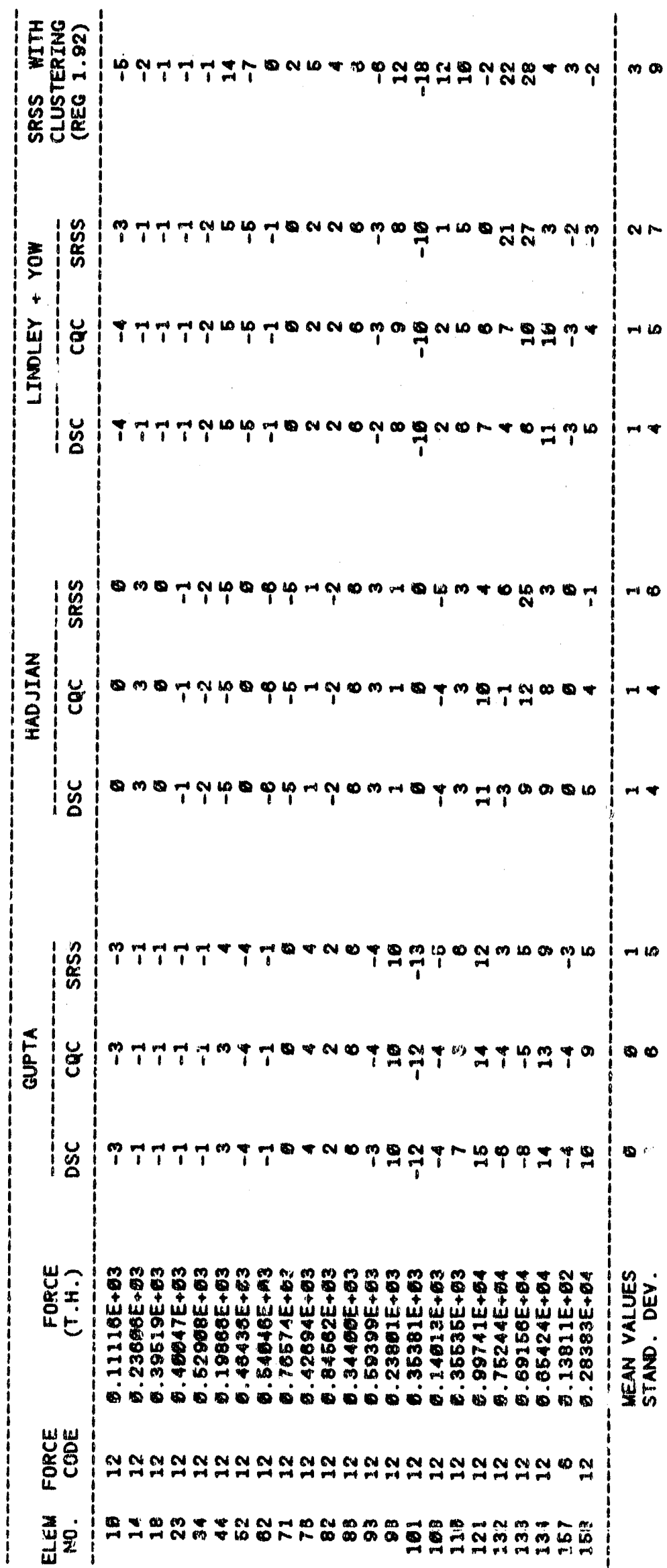

A-119 


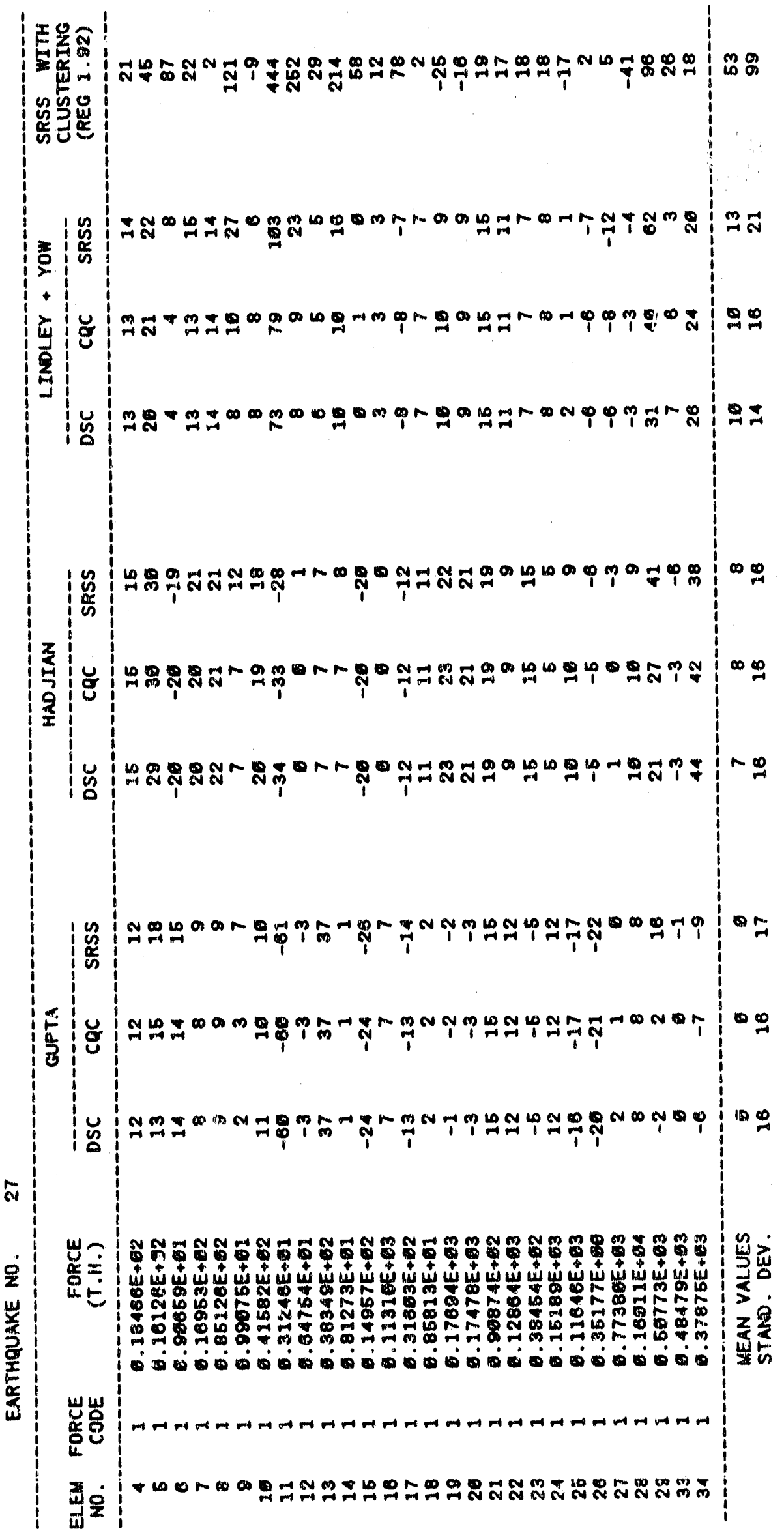


N

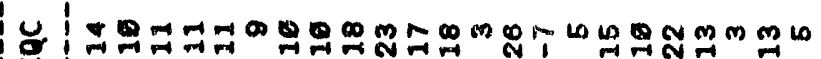




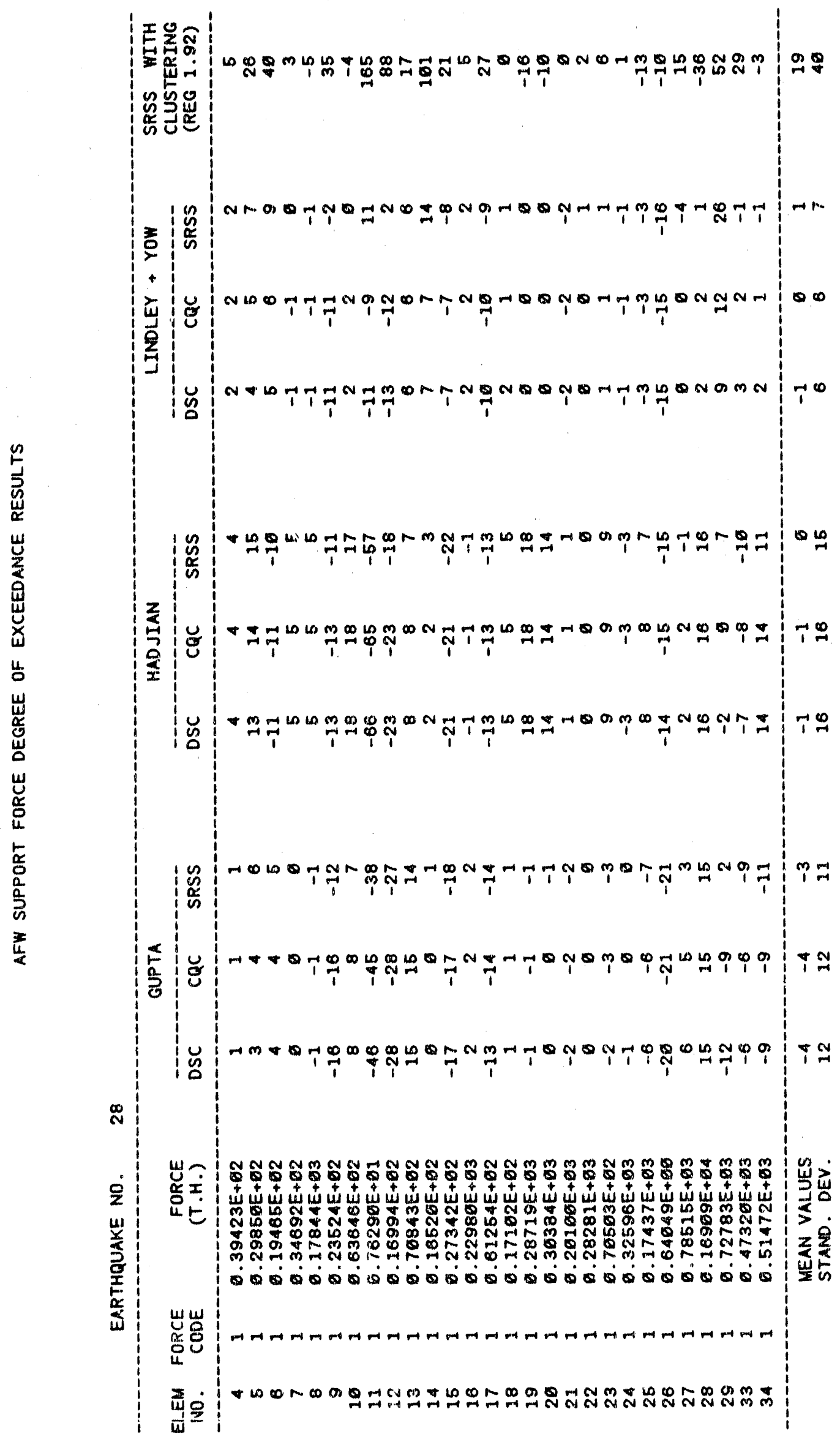




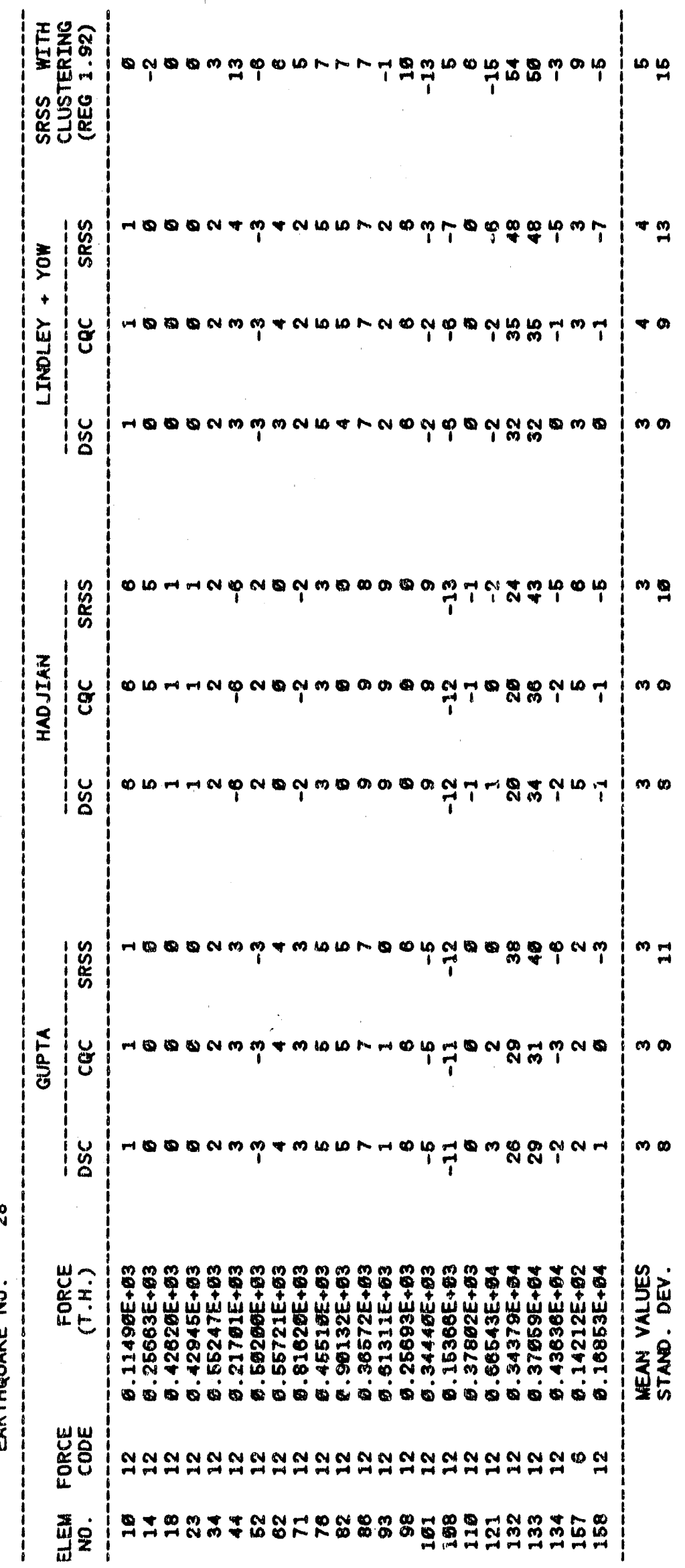




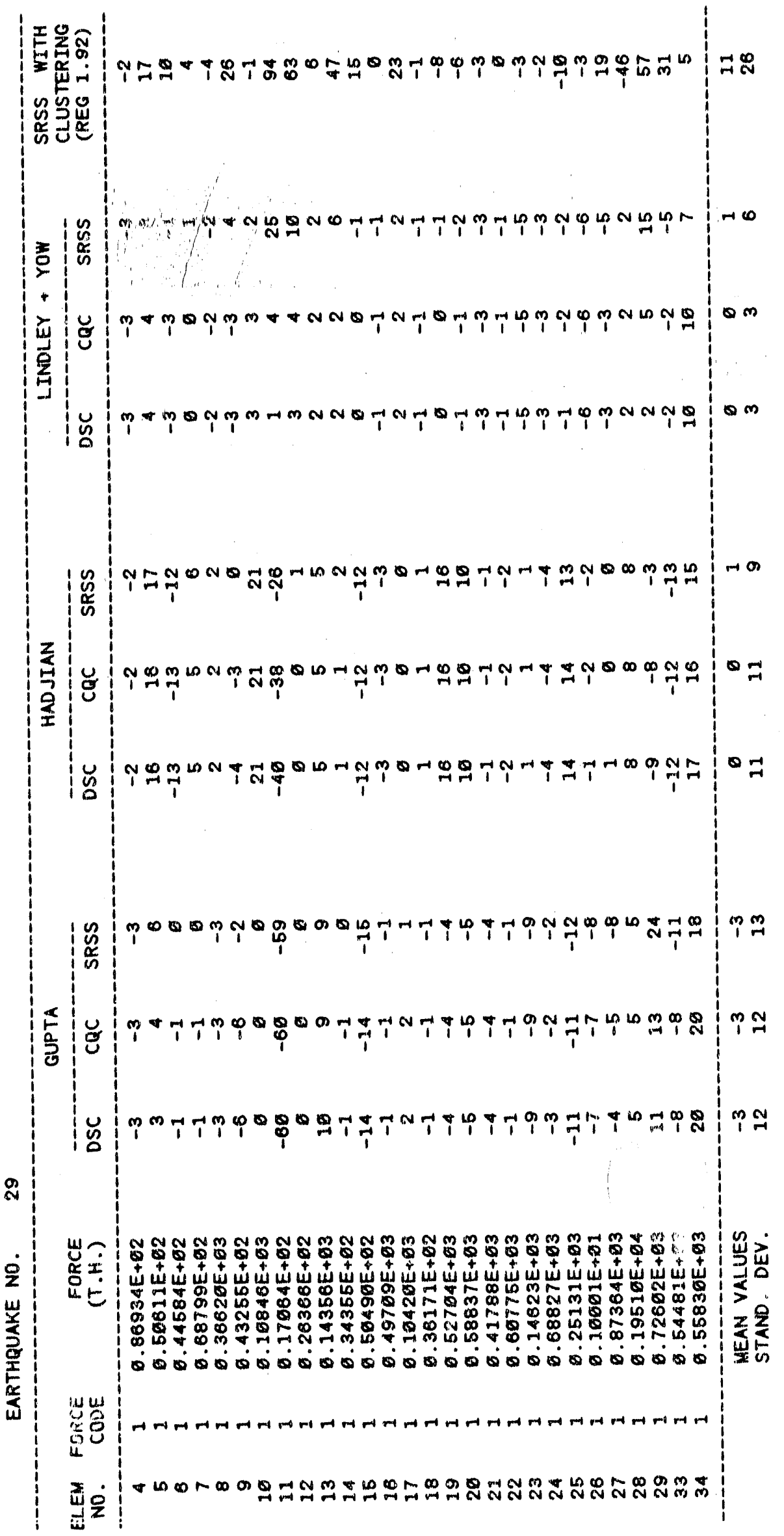




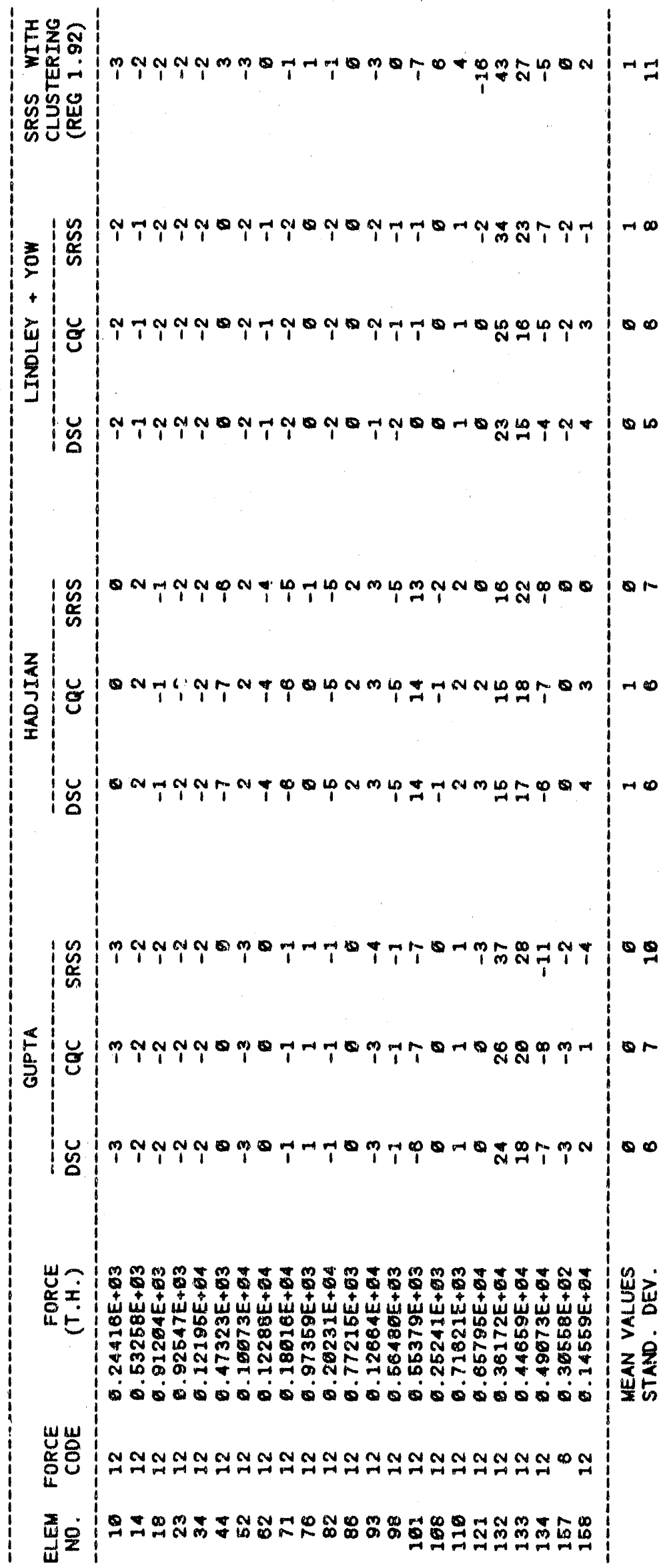




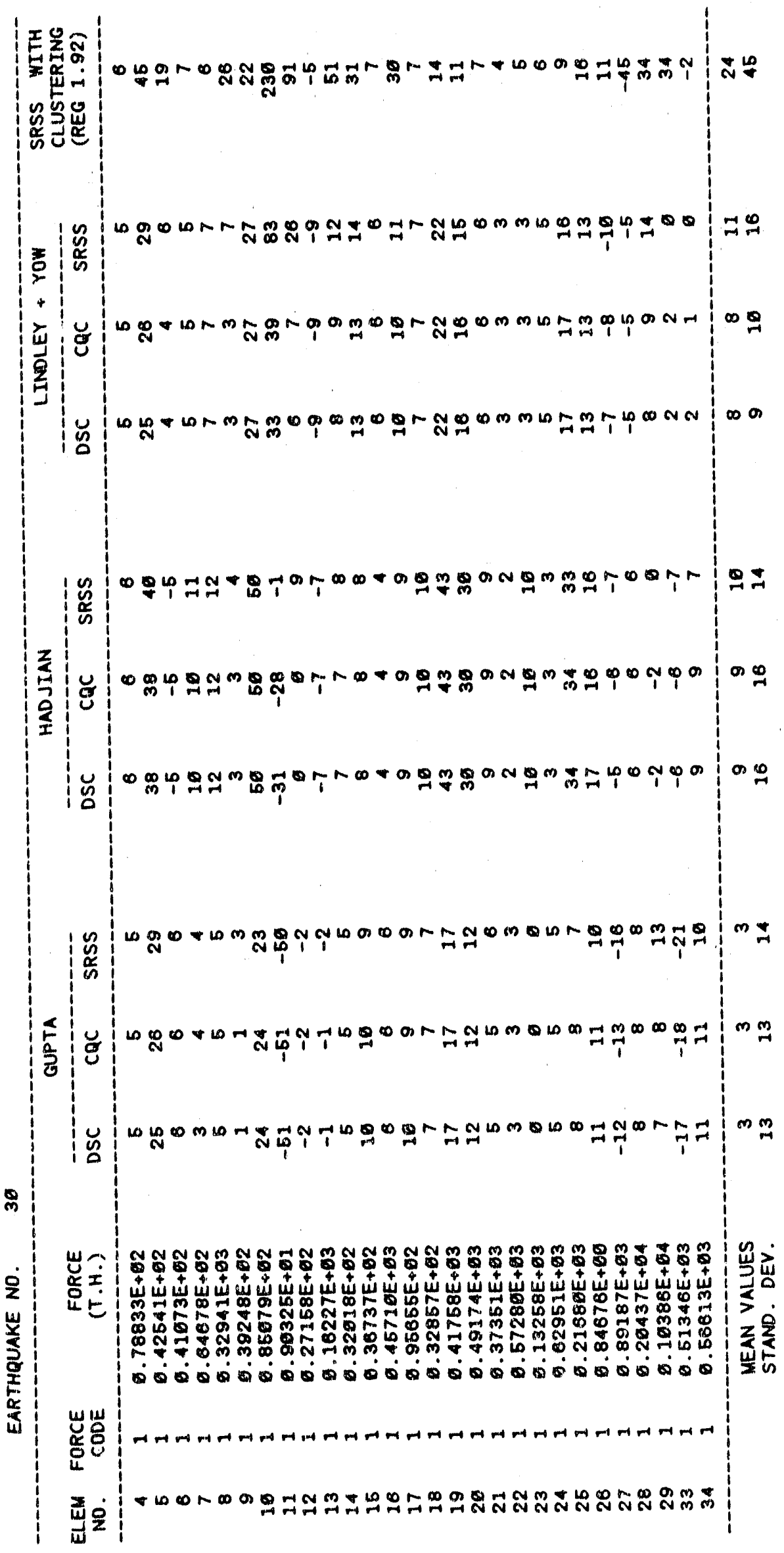




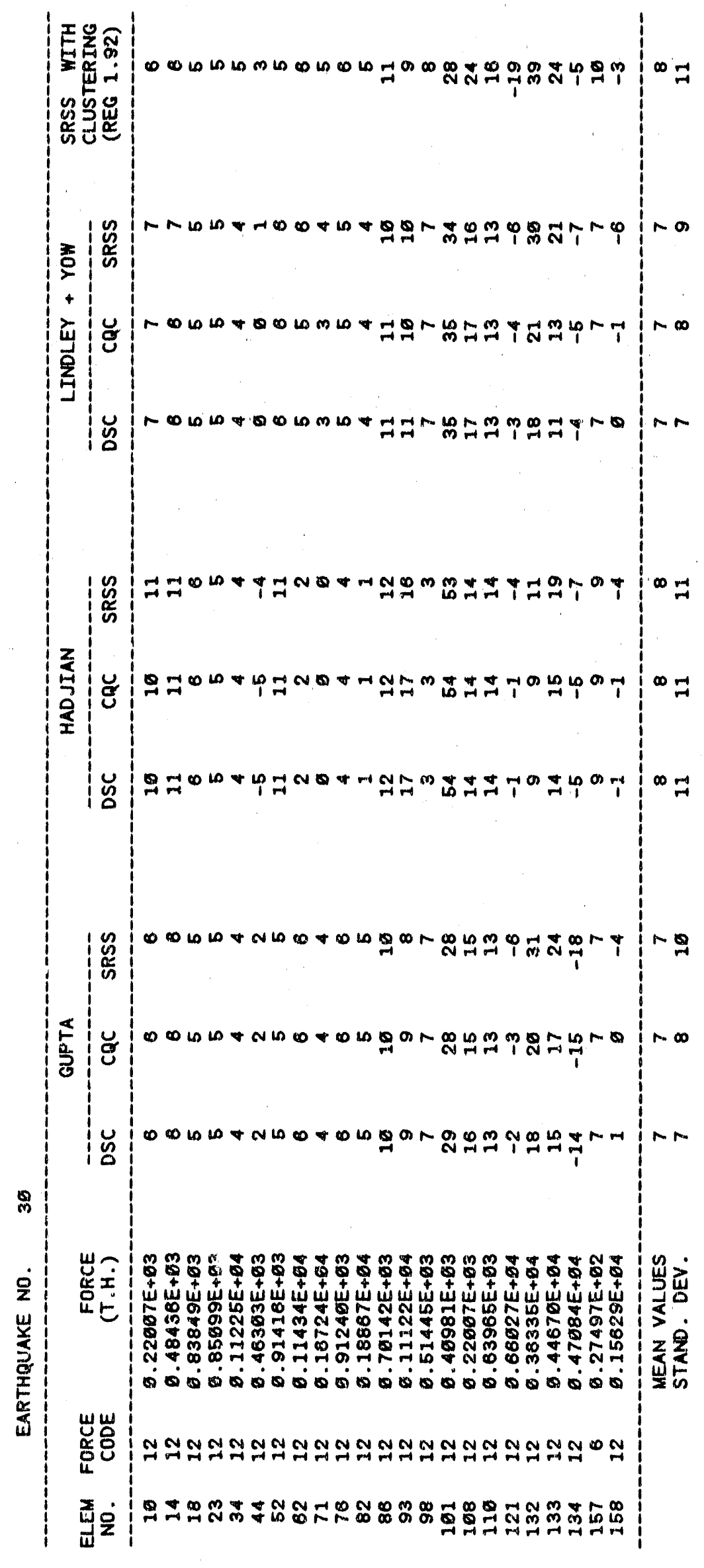




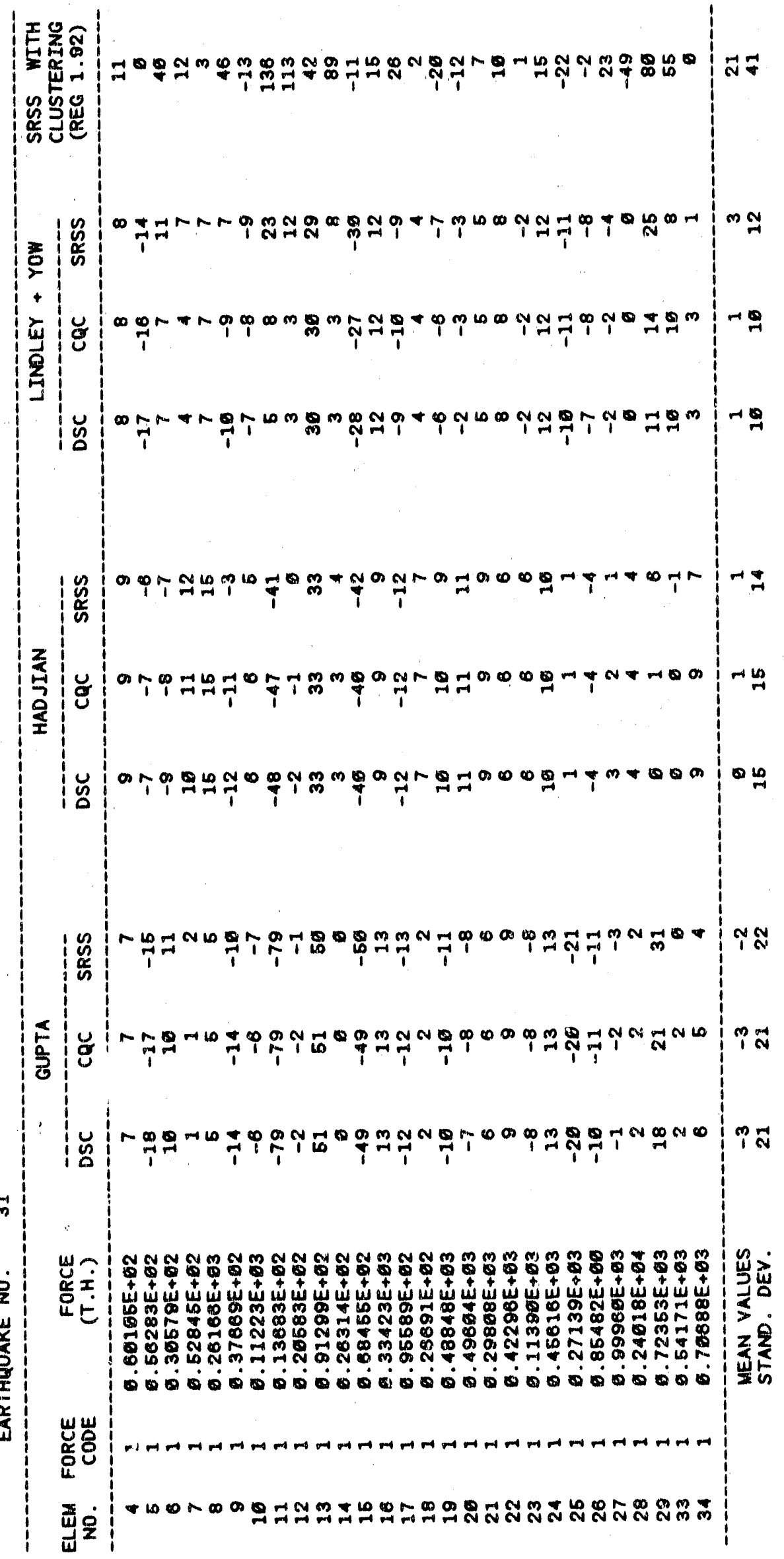




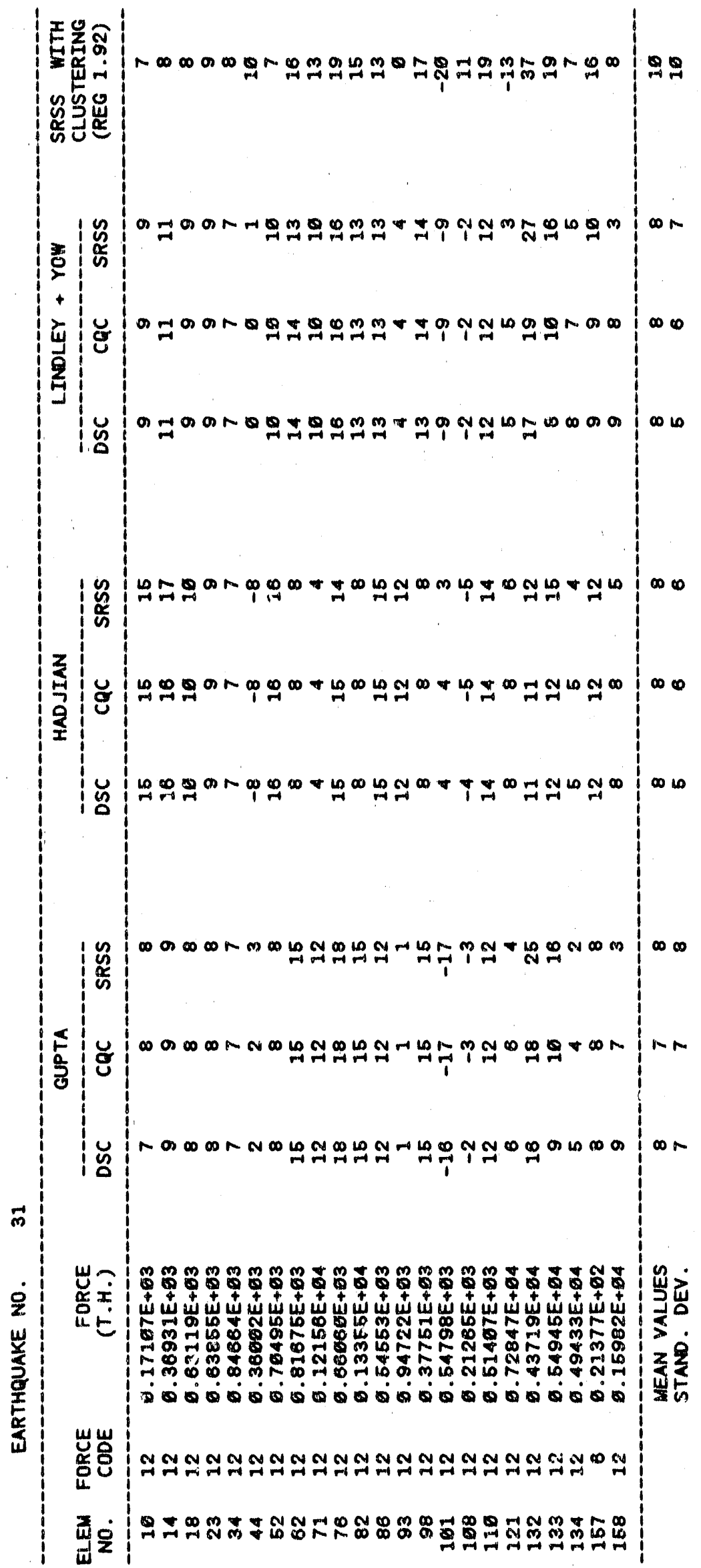




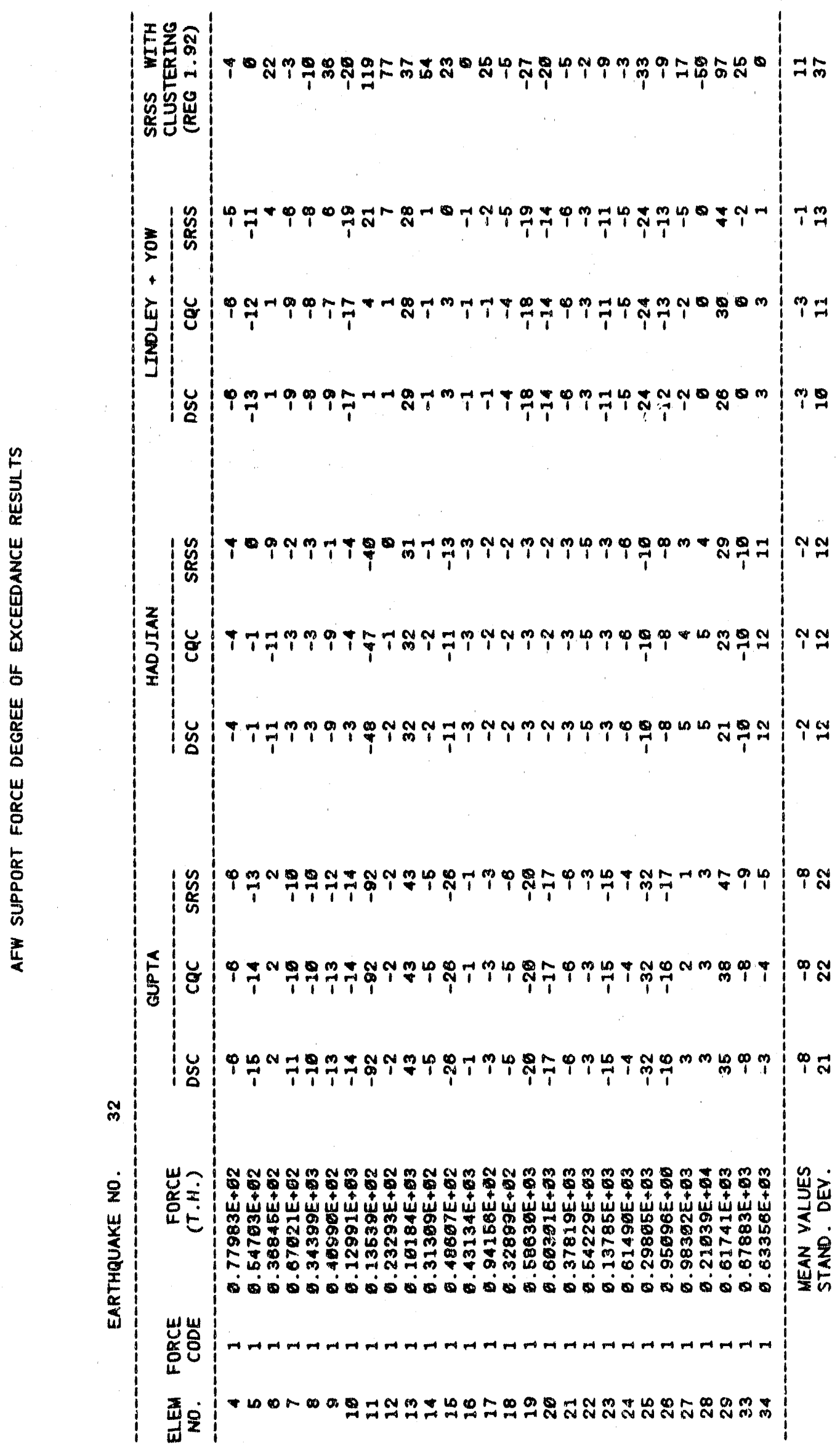




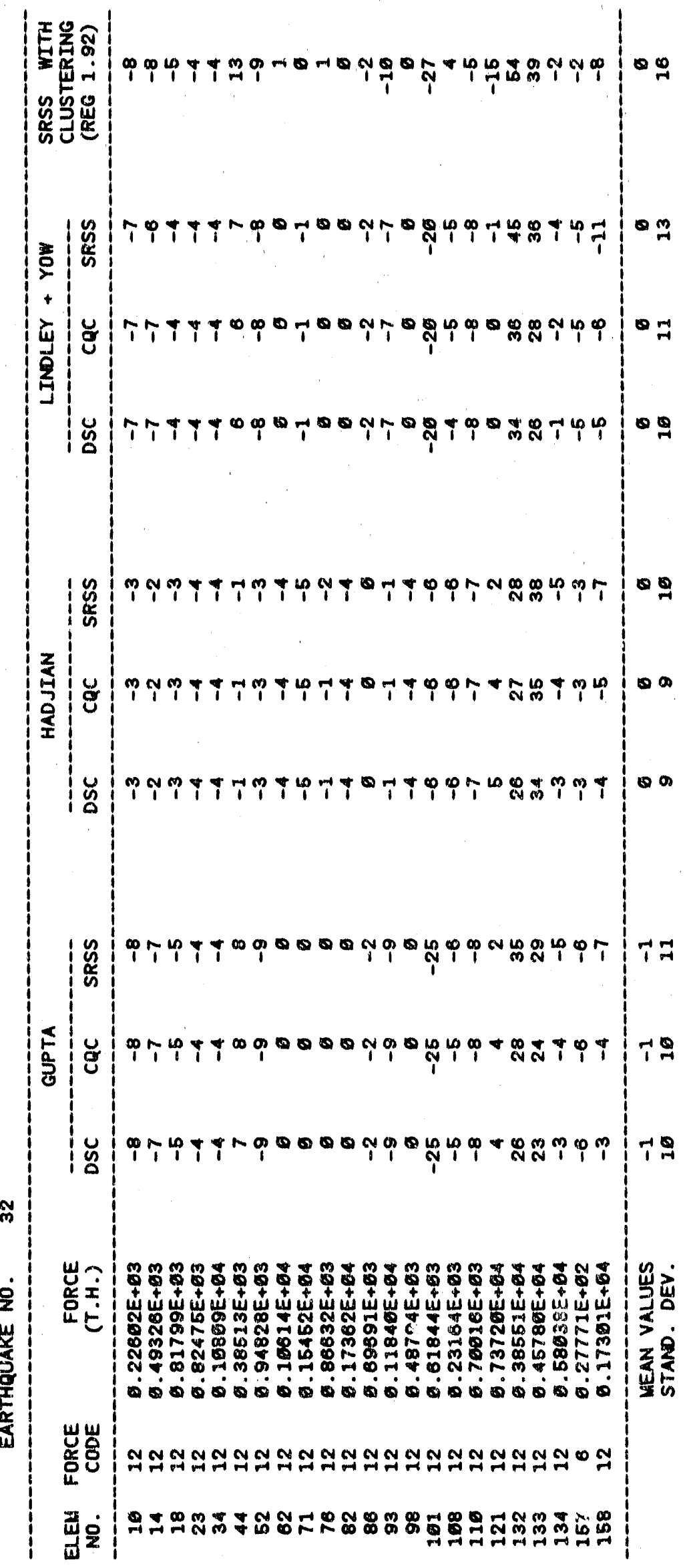




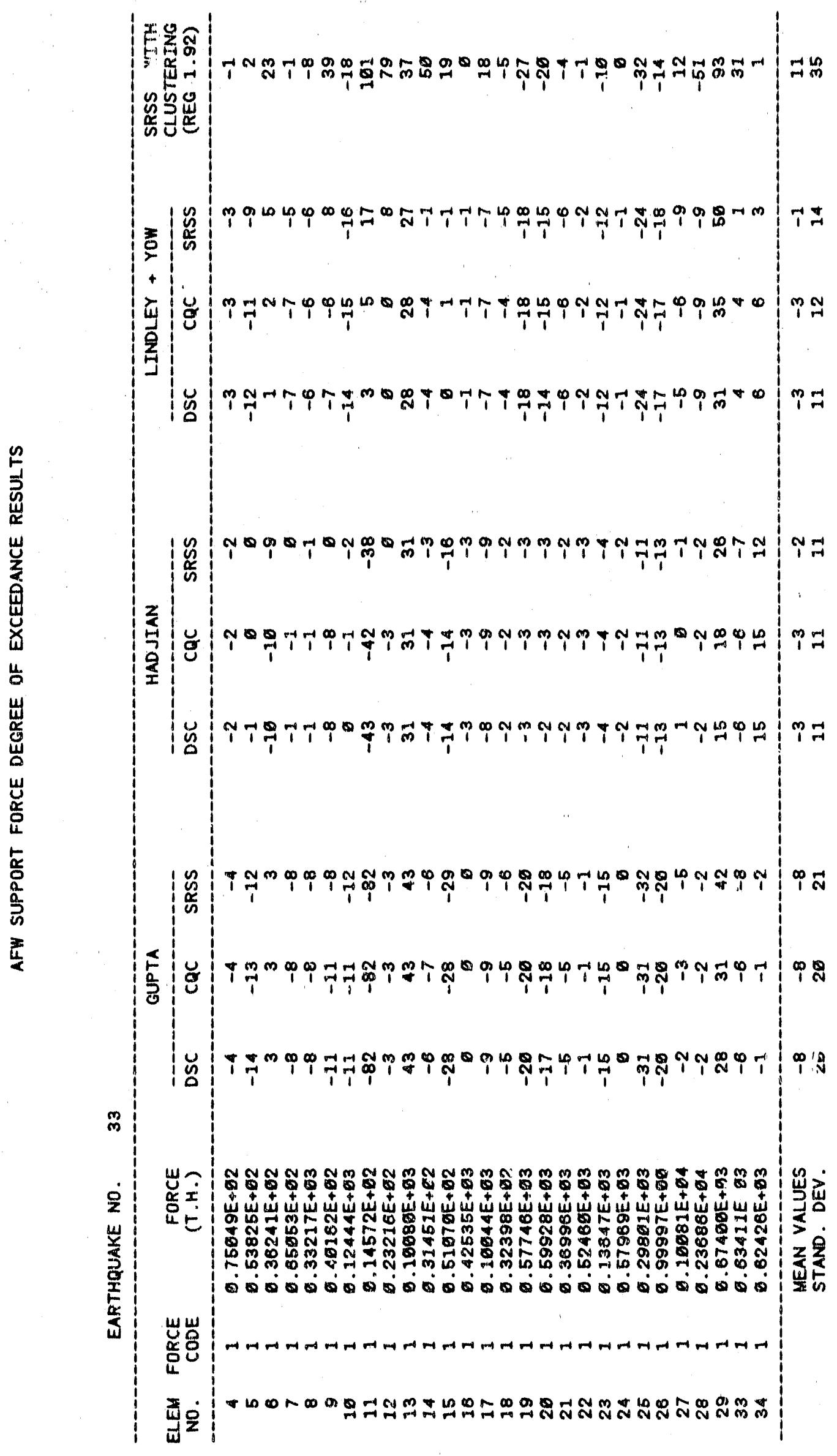

$A-132$ 


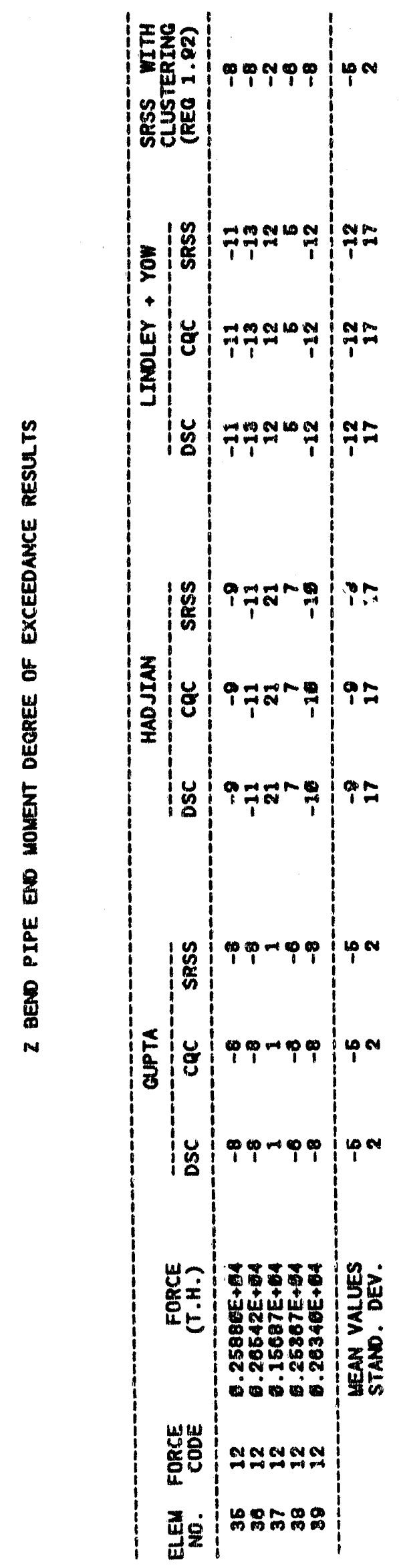

A -135 


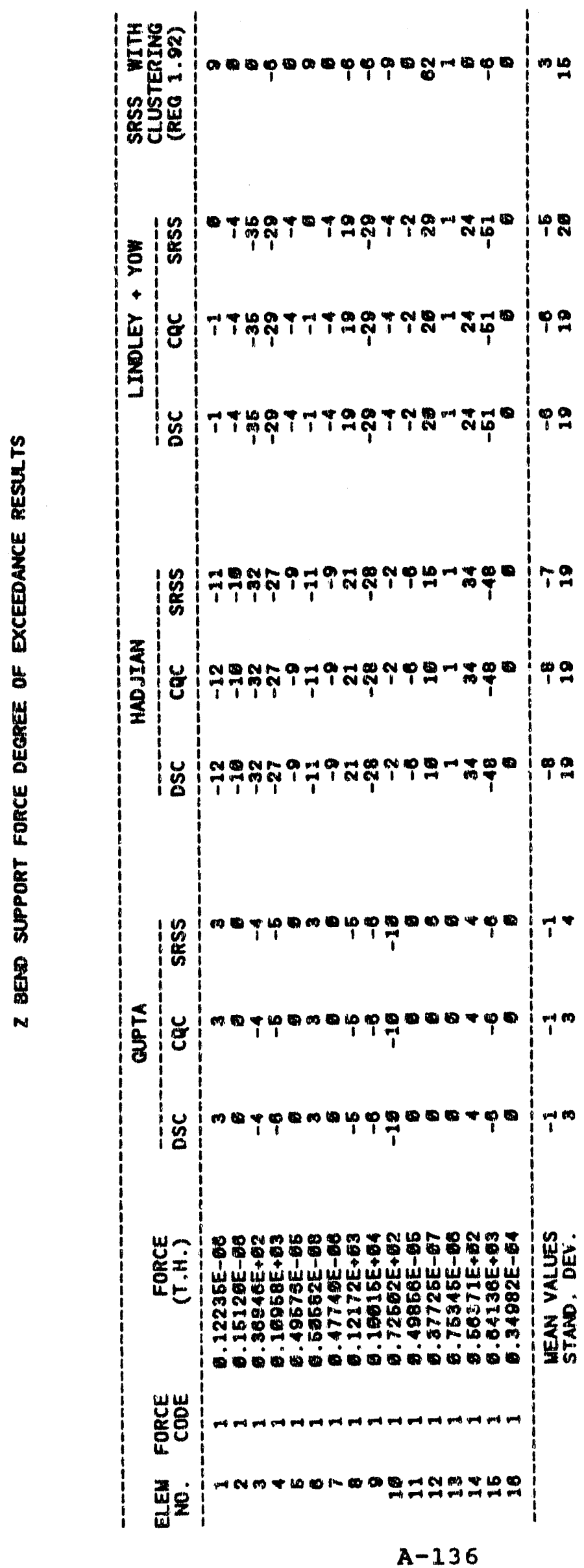




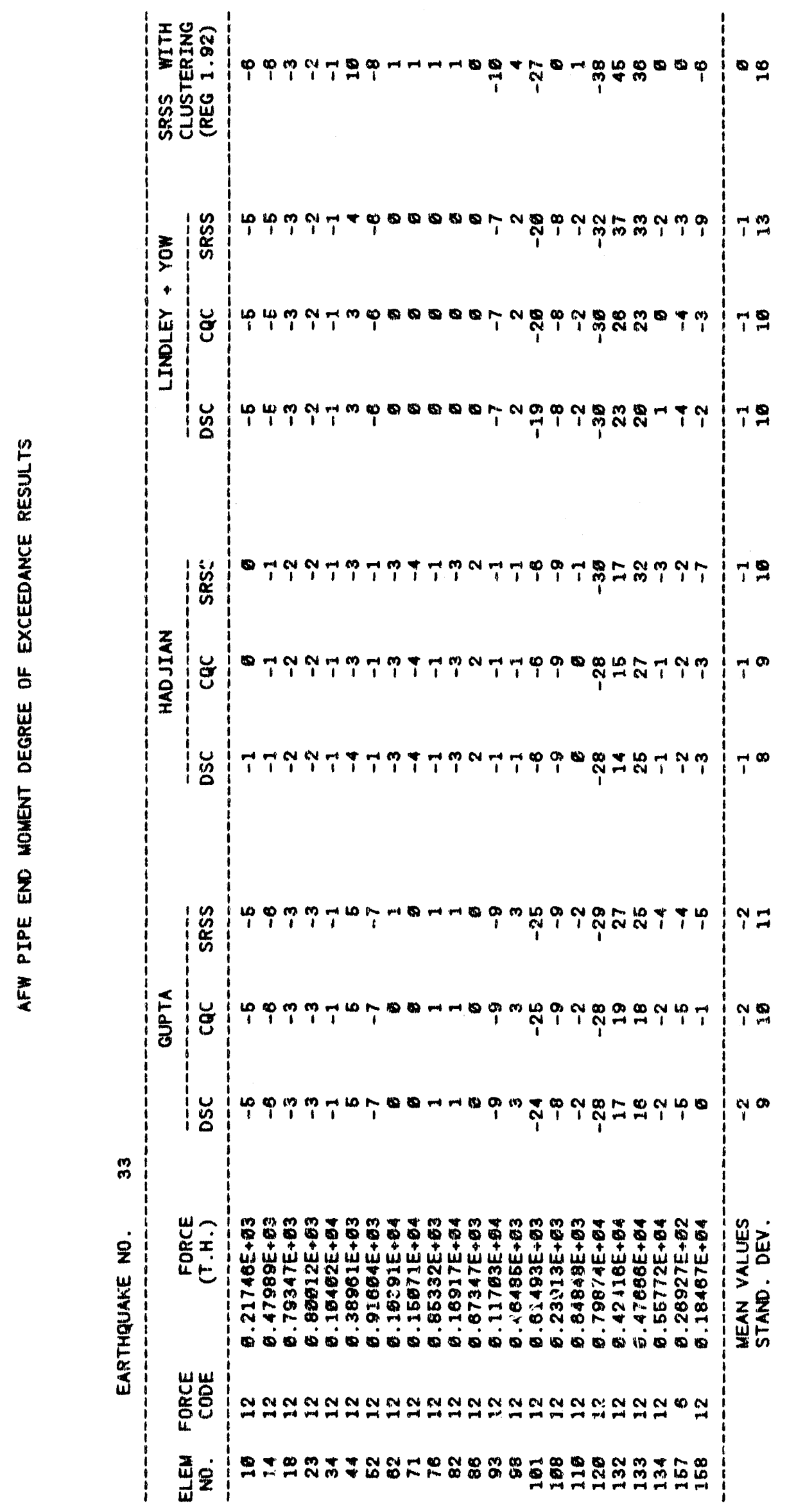

$A-133$ 


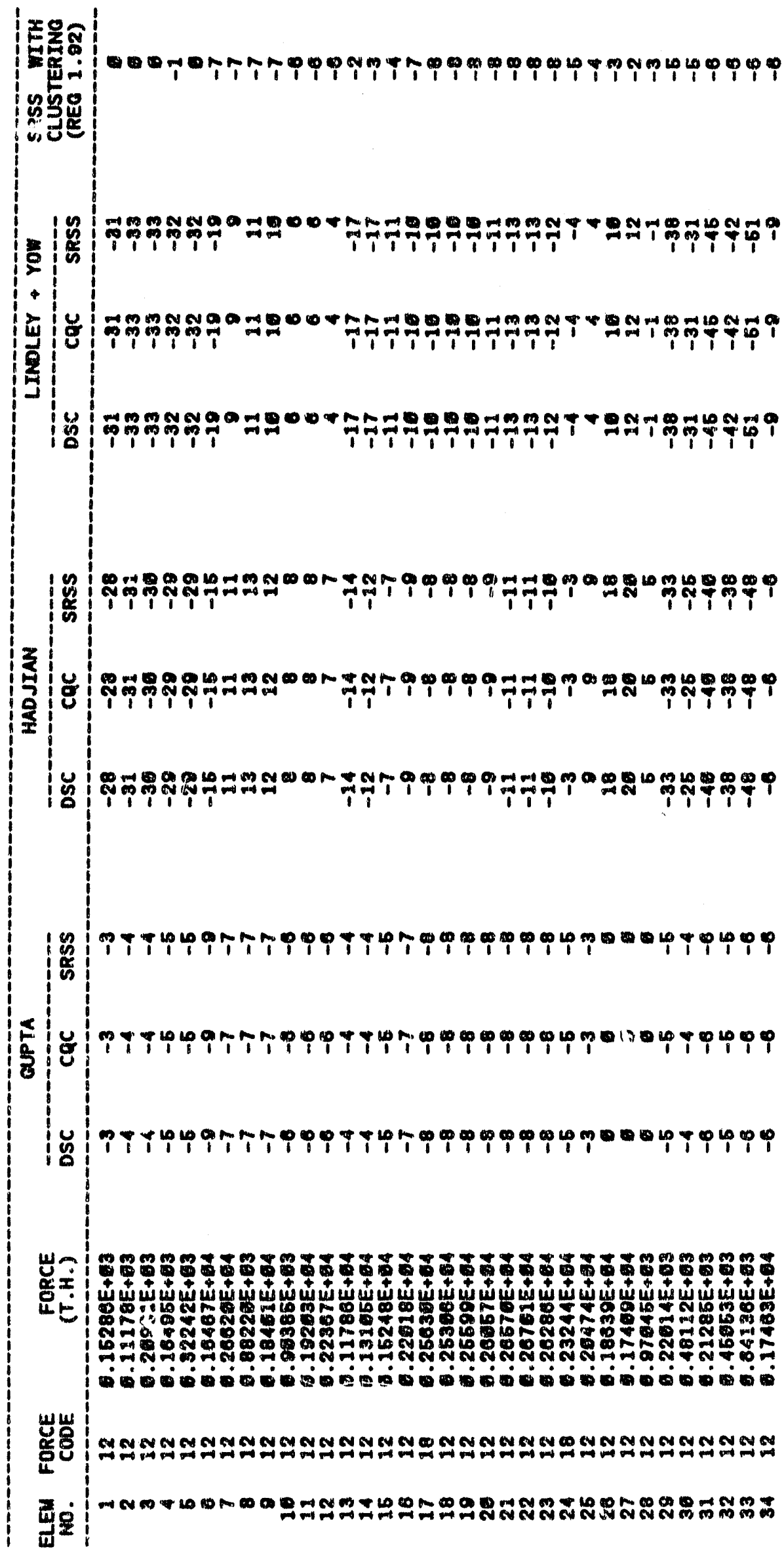

A- 134 


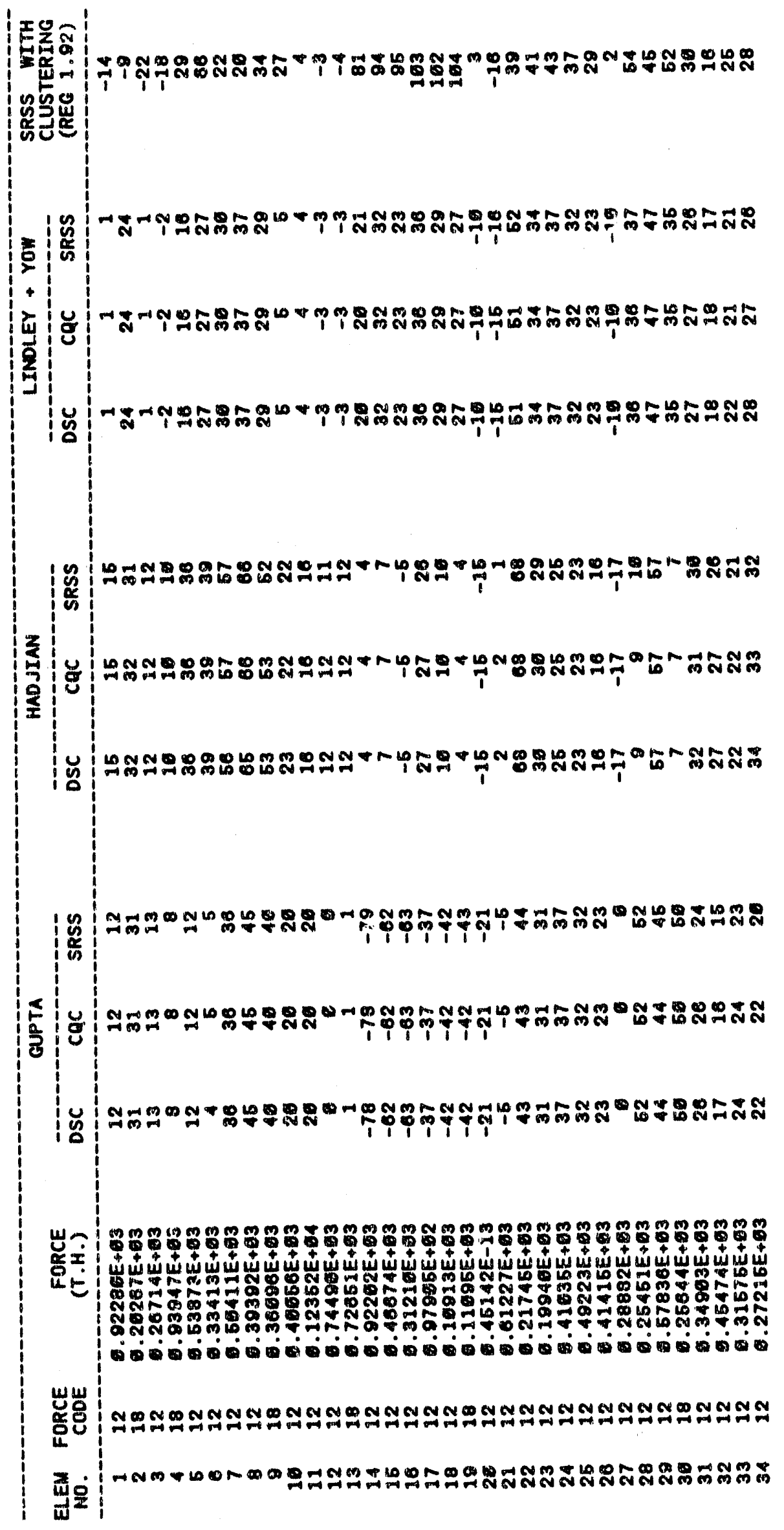




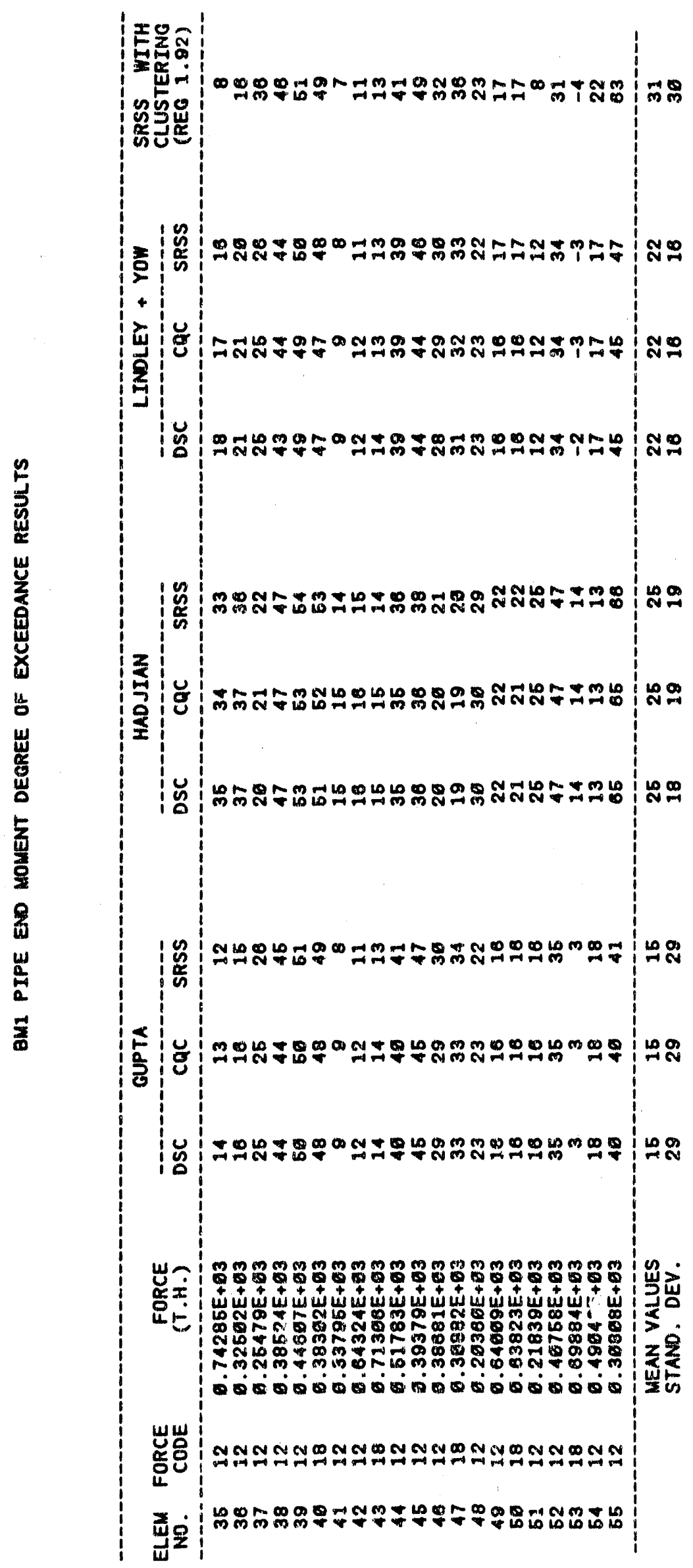

A -138 


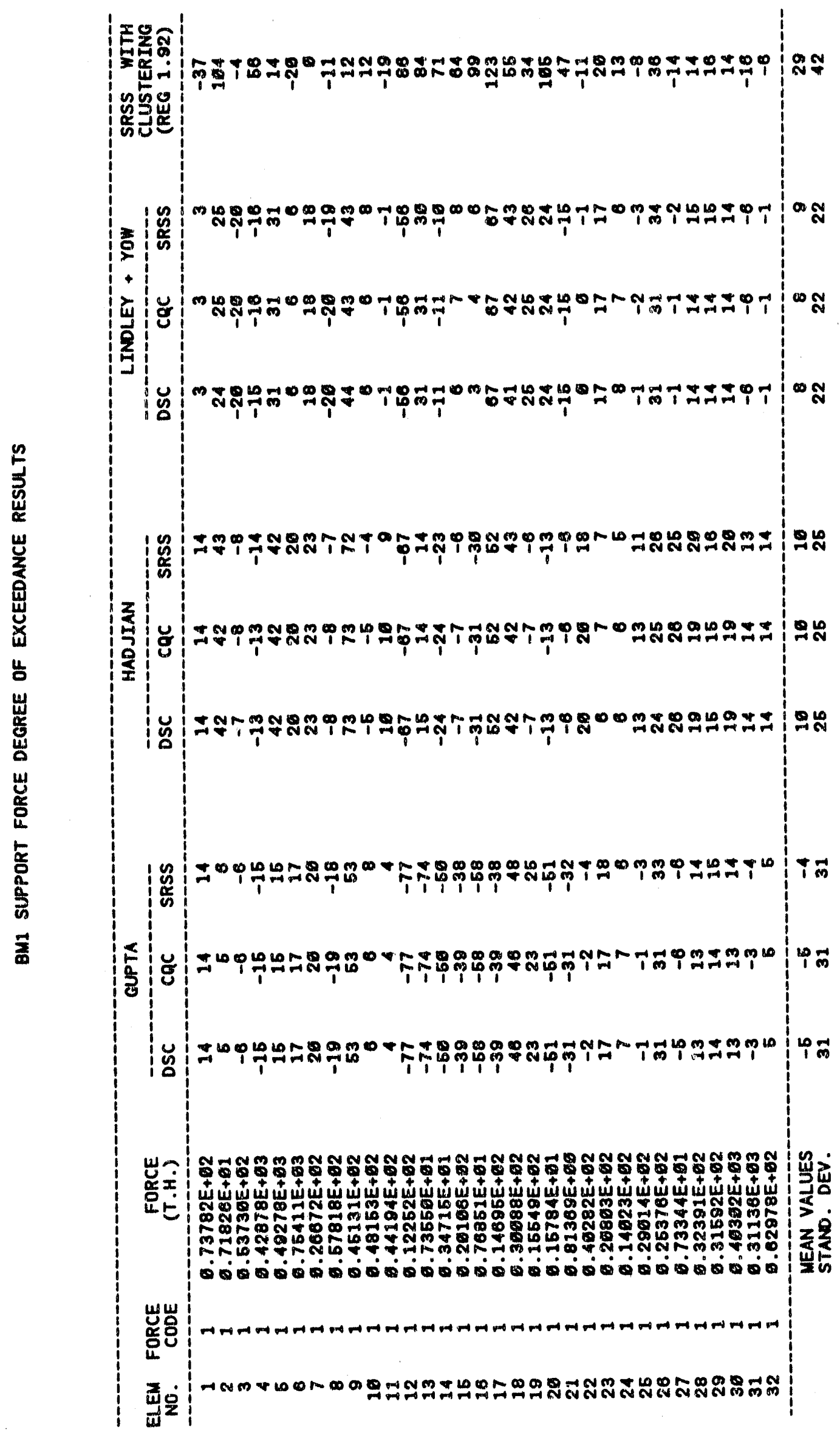

A- 139 


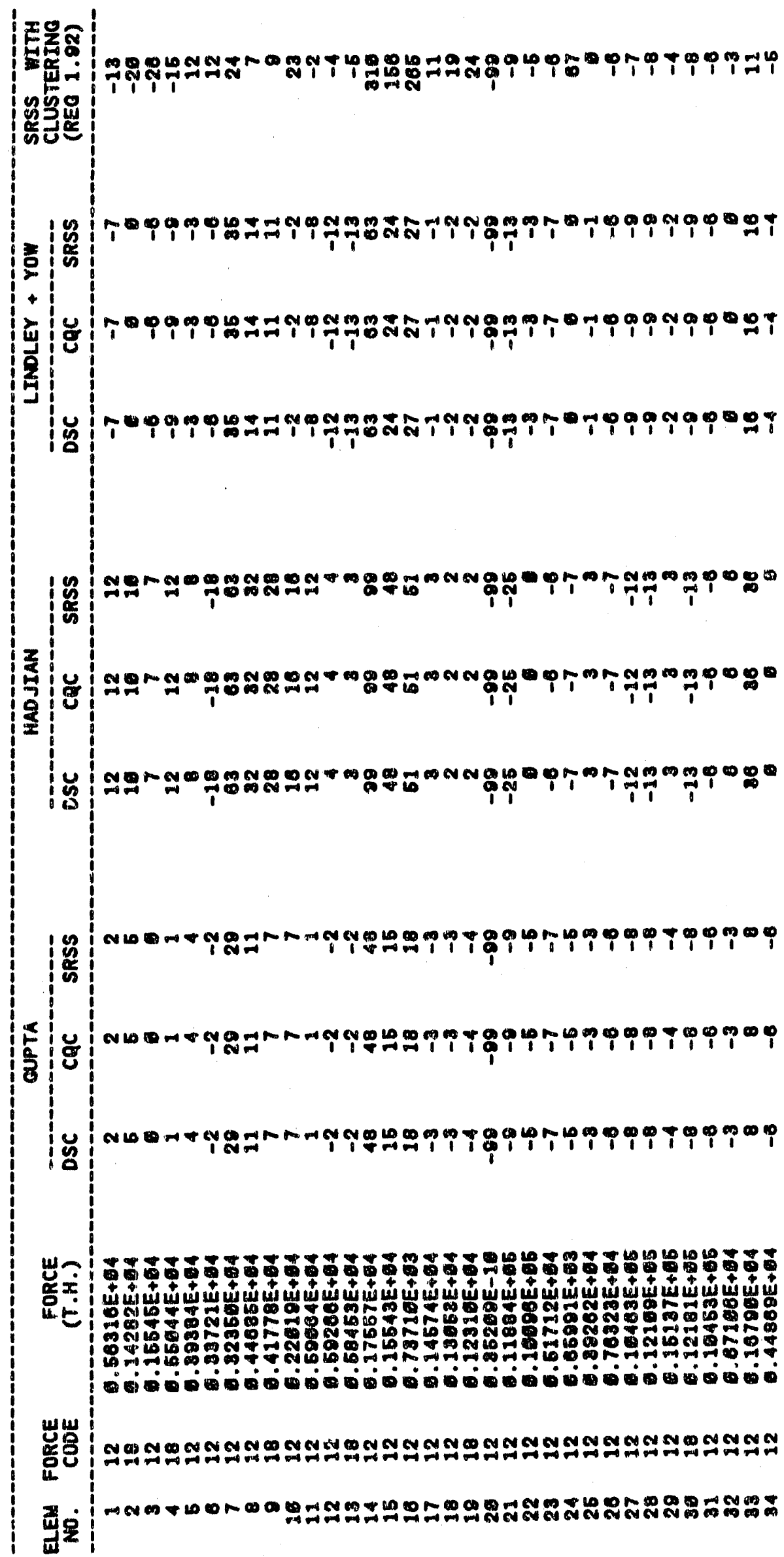




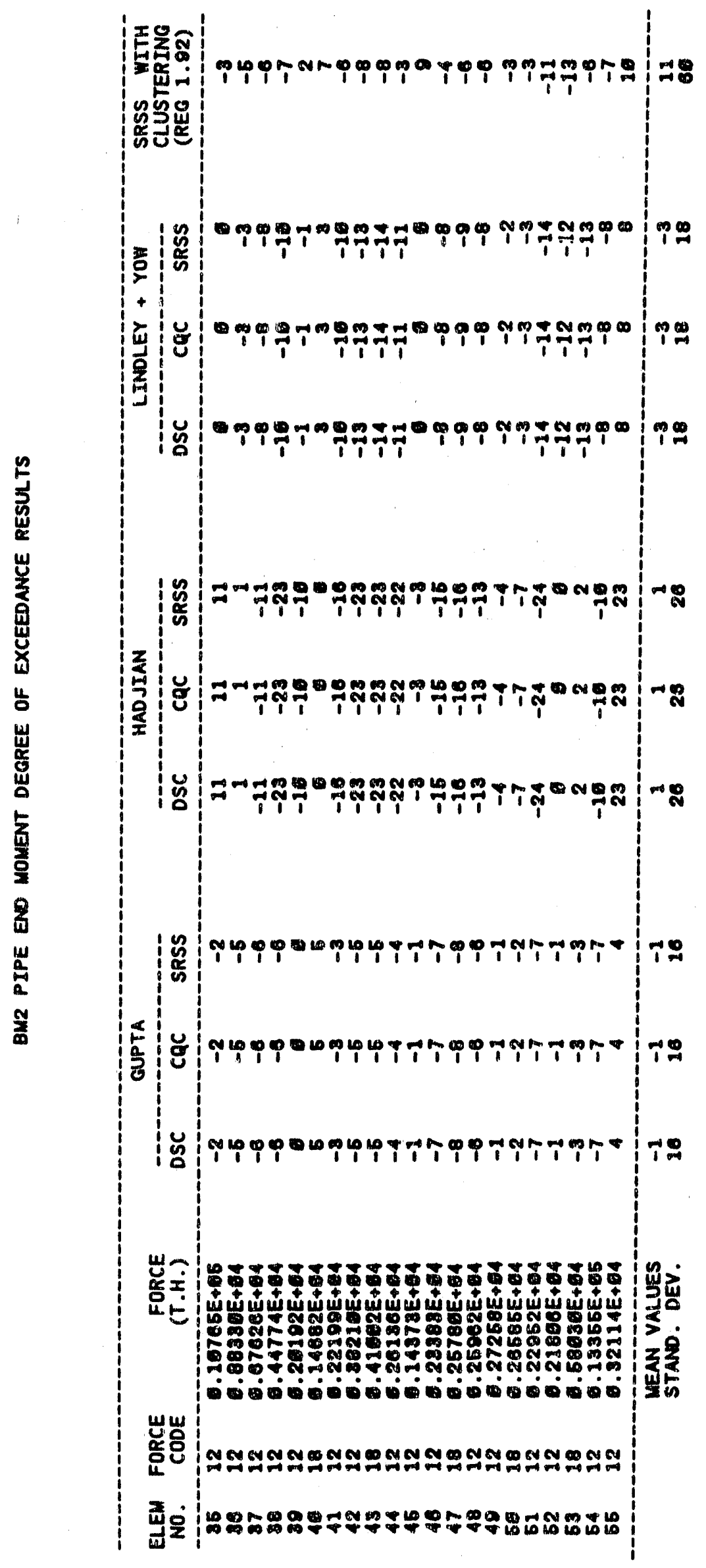

$A-141$ 


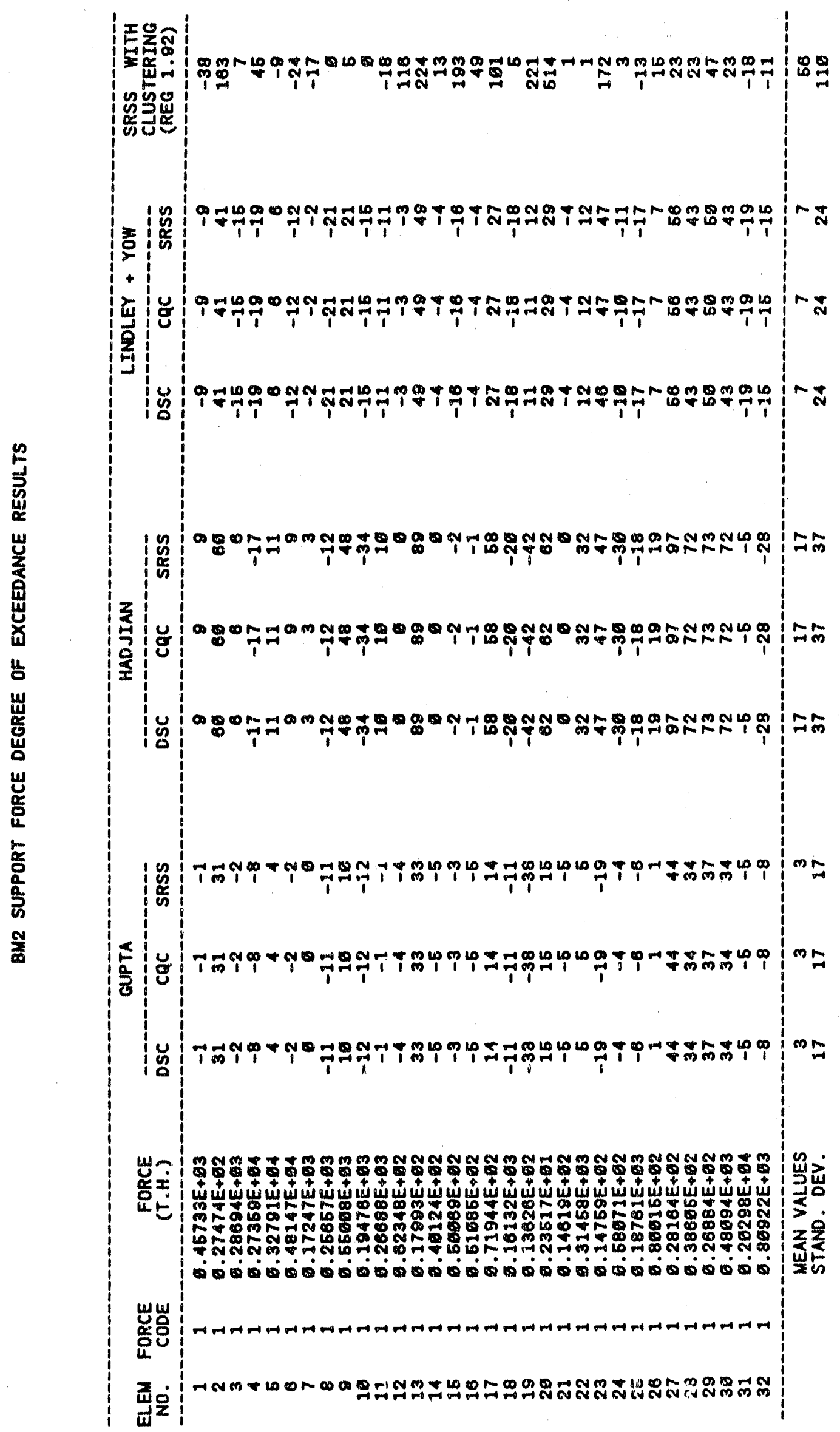

A-142 


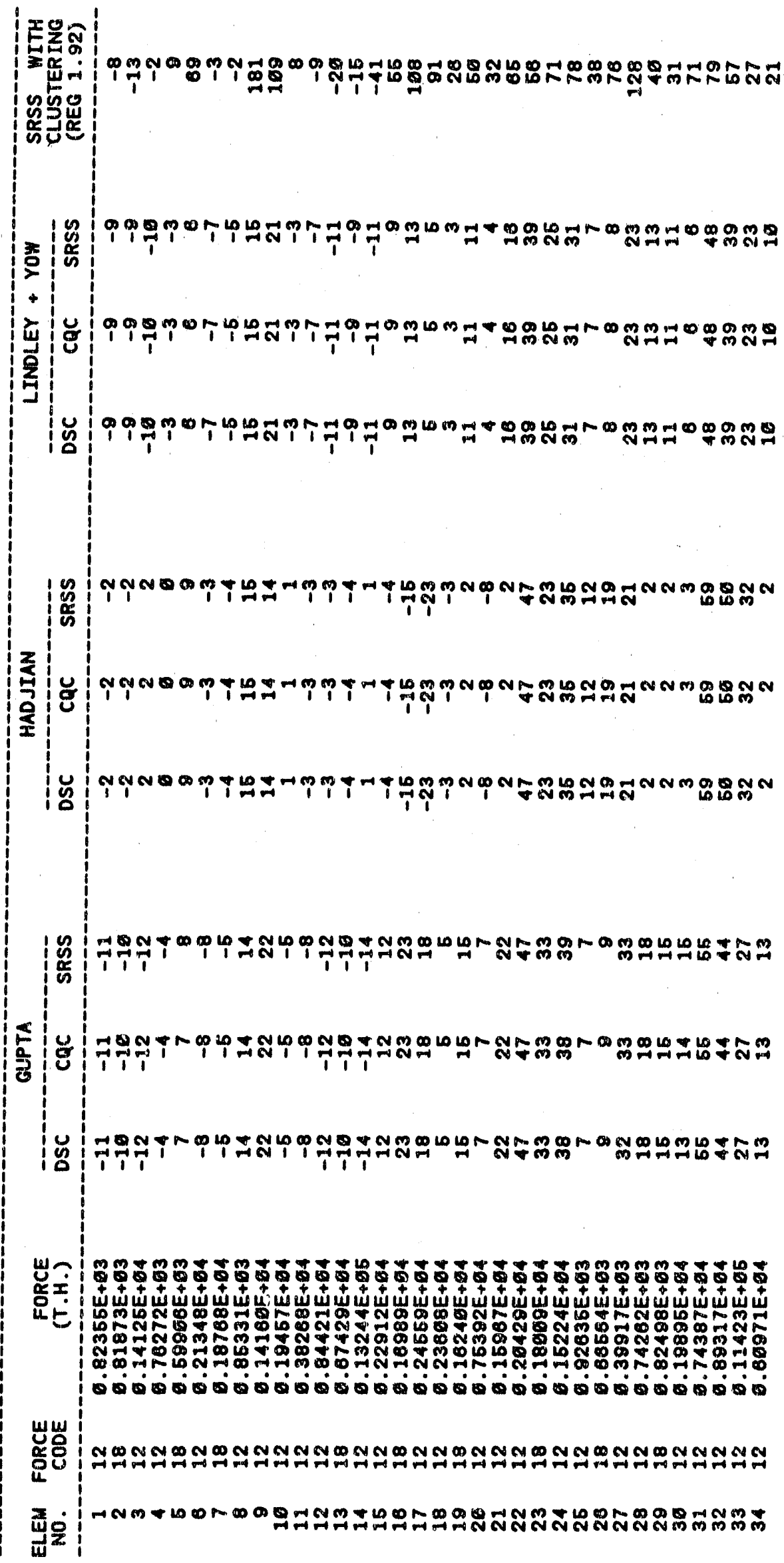




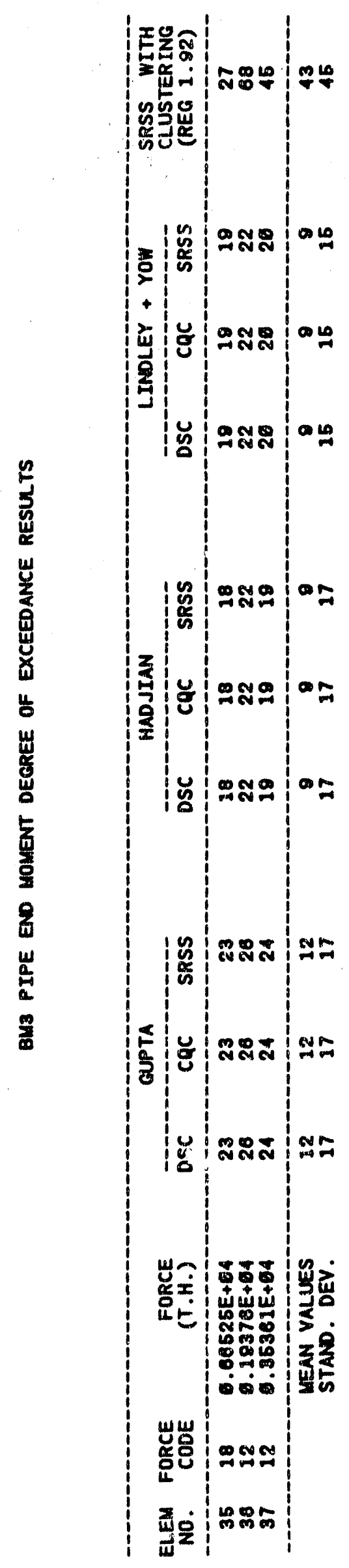




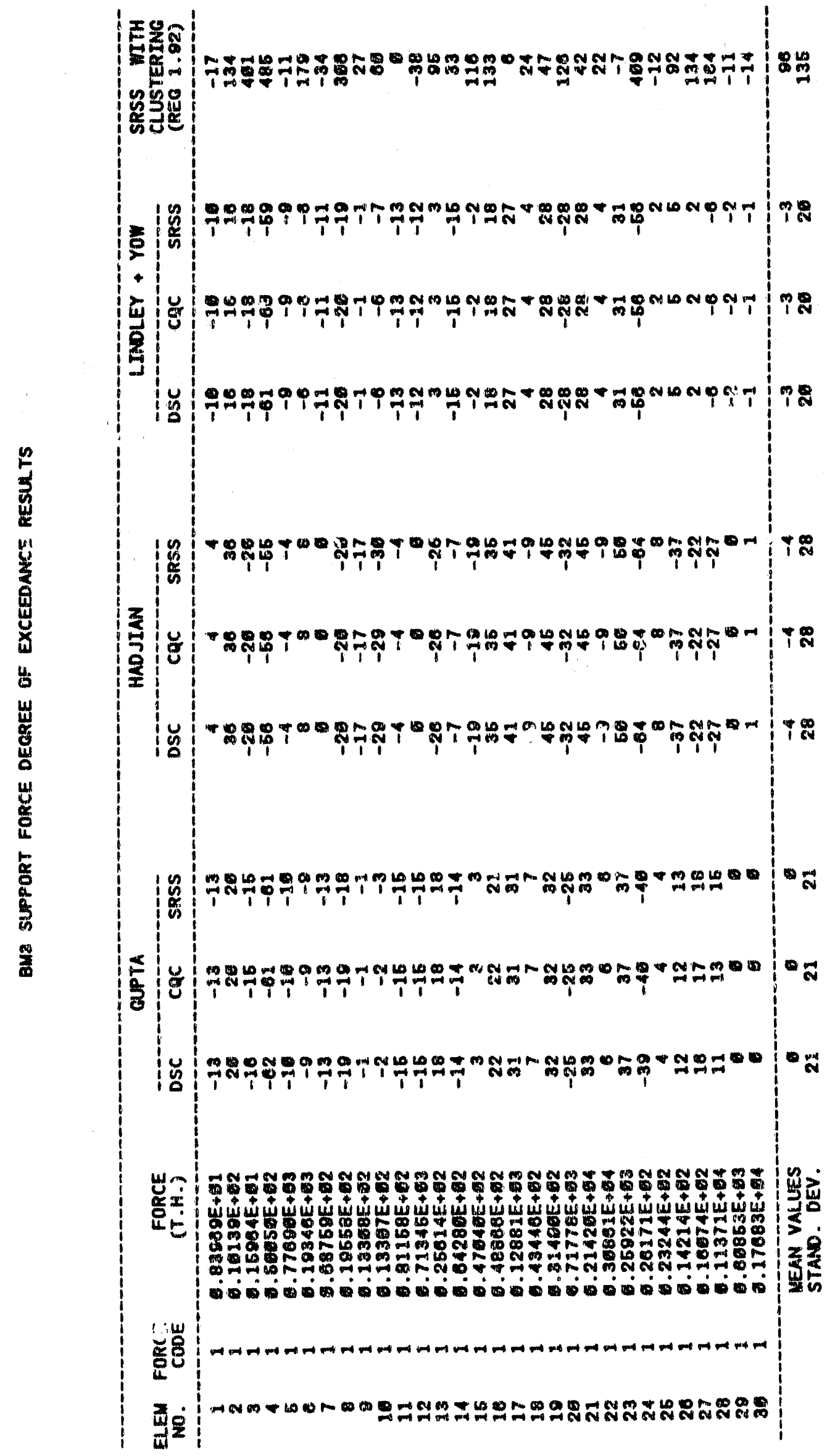


APPENDIX II

$A A-1$ 
A comparison of the Rosenblueth Double Sum Combjnation (DSC) method, the complete Quadratic Combination (CQC) or Der Kiureghian double sum method, SRSS and the absolute sum combination rules was made by Maison, Neuss and Kasai [19]. They analyzed the fifteen story steel moment resisting frame structure of the University of California Medical Center Health Sciences East Building located in San Francisco. Two building models were formulated. The first was the "regular" building in which the cienters of stiffness and mass were coincident. The second wat an irregular building with mass offset from the stiffness center of the building. The regular building did not have interaction between modes with closely spaced frequencies. Therefore, as one would expect the two double sum methods and the SRSS rules gave comparable results, which were also very close to the time history results for the regular building; the absolute sum rule overestimated the response values significantly. In the irregular building, the modes in the two orthogonal directior.s became coupled leading to interacting modes with closely spaced frequencies. Three ground motions were used: San Fernando (Pacoima Dam, SOOE, 1971), Imperial Valley (El Centro, SOOE, 1940), and San Fernando (Orion Blvd., Noow, 1971). The double sum calculations were performed using the modal correlation coefficient from the Rosenblueth-Elorduy equation (DSC), and from the Der Kiureghian equation (CQC). In the former, the effective duration s was taken to be 10 seconds.

A statistical summary of errors is given in Table 1. The earthquake motion was applied in the east and west direction. The response in the north-south direction, and the rotational torque response was generated due to the eccentricity between the mass and the stiffness centers. The parallel east-west responsive values form the two double sum calculations are comparable; the sRSS values have relatively higher errors; the exrors from the absolute sum calculations are the highest. Similar conclusions can be made about the torsional response, except that the absolute sum values now have much higher errors. All the combination rules have the highest errors in the orthogonal north-south response. The double sum method using the Rosenblueth-Elorduy modal correlation coefficient gives the best results. The results from the SRSS and the absolute sum combination rules are literally unacceptable. The orthogonal north-south response values form the san FernandoPacoina Dam excitation are shown in Figures 1,2 and 3. The order of accuracy between different combination rules observed from the figure is the same as that concluded on the basis of Table 1 .

In this example, the response results developed in the time history and the two double s'm response spectrum solutions are less than the results developed in the JRSS response spectrum solution. This occurred kecause in reality the significant modes have opposite signs in the response computation. For another problem, the opposite could be the case when the significant modes have the same sign in the response computation. 
Table 1. Error in Response Spectrum Results with Respect to time History Results.

\begin{tabular}{|c|c|c|c|c|c|}
\hline \multirow[b]{3}{*}{$\begin{array}{l}\text { Response } \\
\text { Quantity }\end{array}$} & \multirow[b]{3}{*}{ Description } & \multicolumn{4}{|c|}{ * Error in Results } \\
\hline & & \multicolumn{2}{|c|}{ Double Sum } & \multirow[t]{2}{*}{ SRSS } & \multirow[t]{2}{*}{ Abs. Sum } \\
\hline & & $\begin{array}{l}\text { Rosenblueth- } \\
\text { Elorduy } \\
\text { (DSC) }\end{array}$ & $\begin{array}{l}\text { Der } \\
\text { Kiureghian } \\
\text { (COC) }\end{array}$ & & \\
\hline \multicolumn{6}{|c|}{ Parallel $(E-W)$ Respanse } \\
\hline Deflection & $\begin{array}{l}\text { Average Error } \\
\text { Maximum Error }\end{array}$ & $\begin{array}{r}7 \\
19\end{array}$ & $\begin{array}{r}6 \\
17\end{array}$ & $\begin{array}{l}18 \\
26\end{array}$ & $\begin{array}{l}27 \\
67\end{array}$ \\
\hline Shear & $\begin{array}{l}\text { Average Error } \\
\text { Maximum Error }\end{array}$ & $\begin{array}{r}8 \\
20\end{array}$ & $\begin{array}{r}8 \\
19\end{array}$ & $\begin{array}{l}22 \\
35\end{array}$ & $\begin{array}{r}49 \\
122\end{array}$ \\
\hline $\begin{array}{l}\text { Overturning } \\
\text { Moment }\end{array}$ & $\begin{array}{l}\text { Average Error } \\
\text { Maximum Error }\end{array}$ & $\begin{array}{r}6 \\
18\end{array}$ & $\begin{array}{r}7 \\
18\end{array}$ & $\begin{array}{l}25 \\
34\end{array}$ & $\begin{array}{r}39 \\
120\end{array}$ \\
\hline \multicolumn{6}{|c|}{ Orthogonal $(\mathrm{N}-\mathrm{S})$ Response } \\
\hline Deflection & $\begin{array}{l}\text { Average Error } \\
\text { Maximun Error }\end{array}$ & $\begin{array}{l}18 \\
33\end{array}$ & $\begin{array}{l}32 \\
67\end{array}$ & $\begin{array}{l}251 \\
350\end{array}$ & $\begin{array}{l}491 \\
800\end{array}$ \\
\hline binar & $\begin{array}{l}\text { Average Error } \\
\text { Maximum Error }\end{array}$ & $\begin{array}{l}17 \\
31\end{array}$ & $\begin{array}{l}24 \\
55\end{array}$ & $\begin{array}{l}217 \\
307\end{array}$ & $\begin{array}{l}528 \\
661\end{array}$ \\
\hline $\begin{array}{l}\text { Overturning } \\
\text { Moment }\end{array}$ & $\begin{array}{l}\text { Average Error } \\
\text { Maximum Error }\end{array}$ & $\begin{array}{l}16 \\
25\end{array}$ & $\begin{array}{l}25 \\
51\end{array}$ & $\begin{array}{l}218 \\
299\end{array}$ & $\begin{array}{l}520 \\
658\end{array}$ \\
\hline \multicolumn{6}{|c|}{ Torsional Response } \\
\hline Torques & $\begin{array}{l}\text { Average Error } \\
\text { Maximum Error }\end{array}$ & $\begin{array}{r}9 \\
27\end{array}$ & $\begin{array}{r}7 \\
26\end{array}$ & $\begin{array}{l}13 \\
40\end{array}$ & $\begin{array}{l}137 \\
288\end{array}$ \\
\hline
\end{tabular}




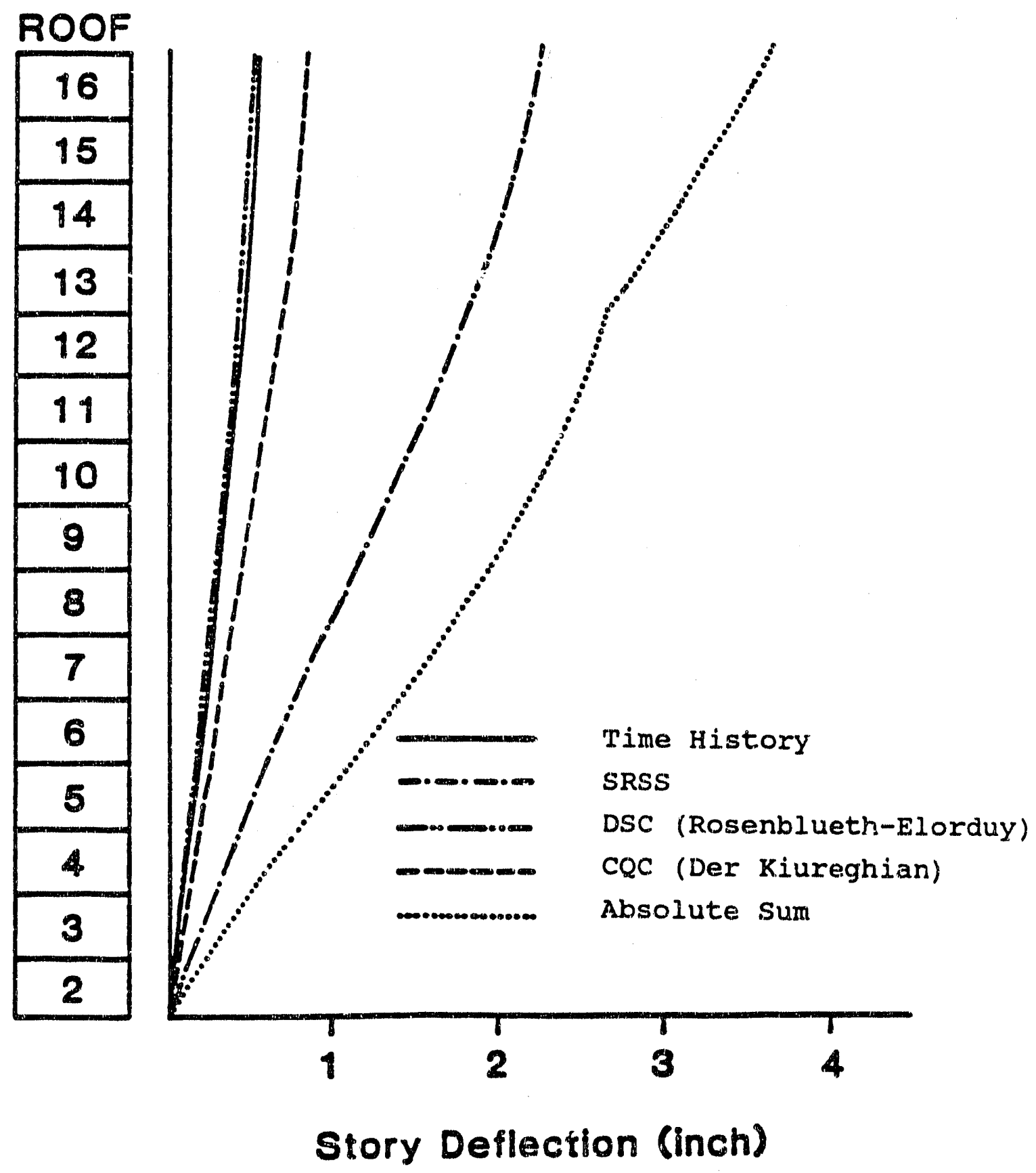

Figure 1 Comparison of Modal Combination Rules Story Deflections

$A A-3$ 


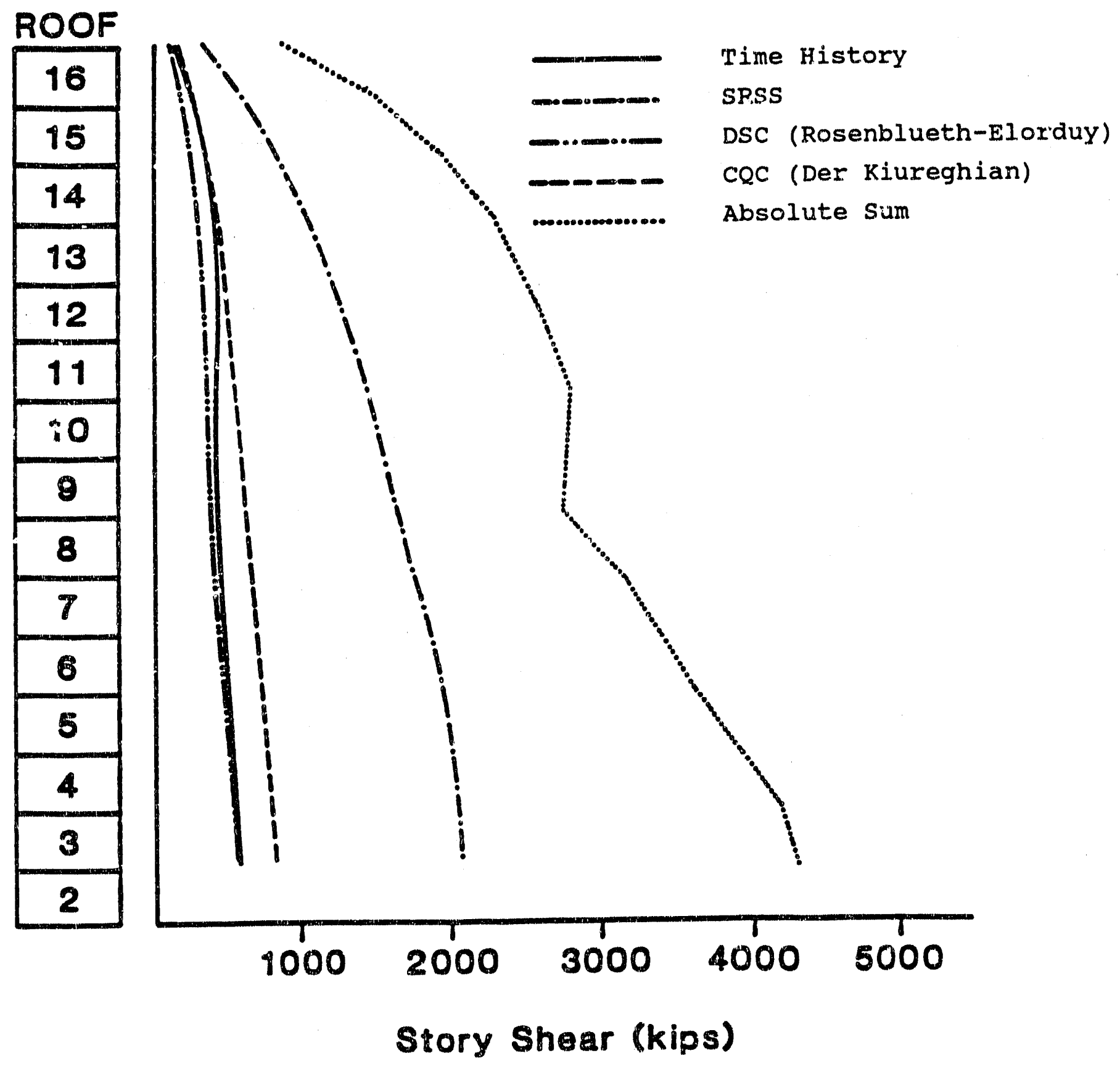

Figure 2 Comparison of Modal Combination Rules
Story Shears

$A A-4$ 


ROOF
\begin{tabular}{|c|}
\hline 16 \\
\hline 15 \\
\hline 14 \\
\hline 13 \\
\hline 12 \\
\hline 11 \\
\hline 10 \\
\hline 9 \\
\hline 8 \\
\hline 7 \\
\hline 6 \\
\hline 5 \\
\hline 4 \\
\hline 3 \\
\hline 2 \\
\hline
\end{tabular}

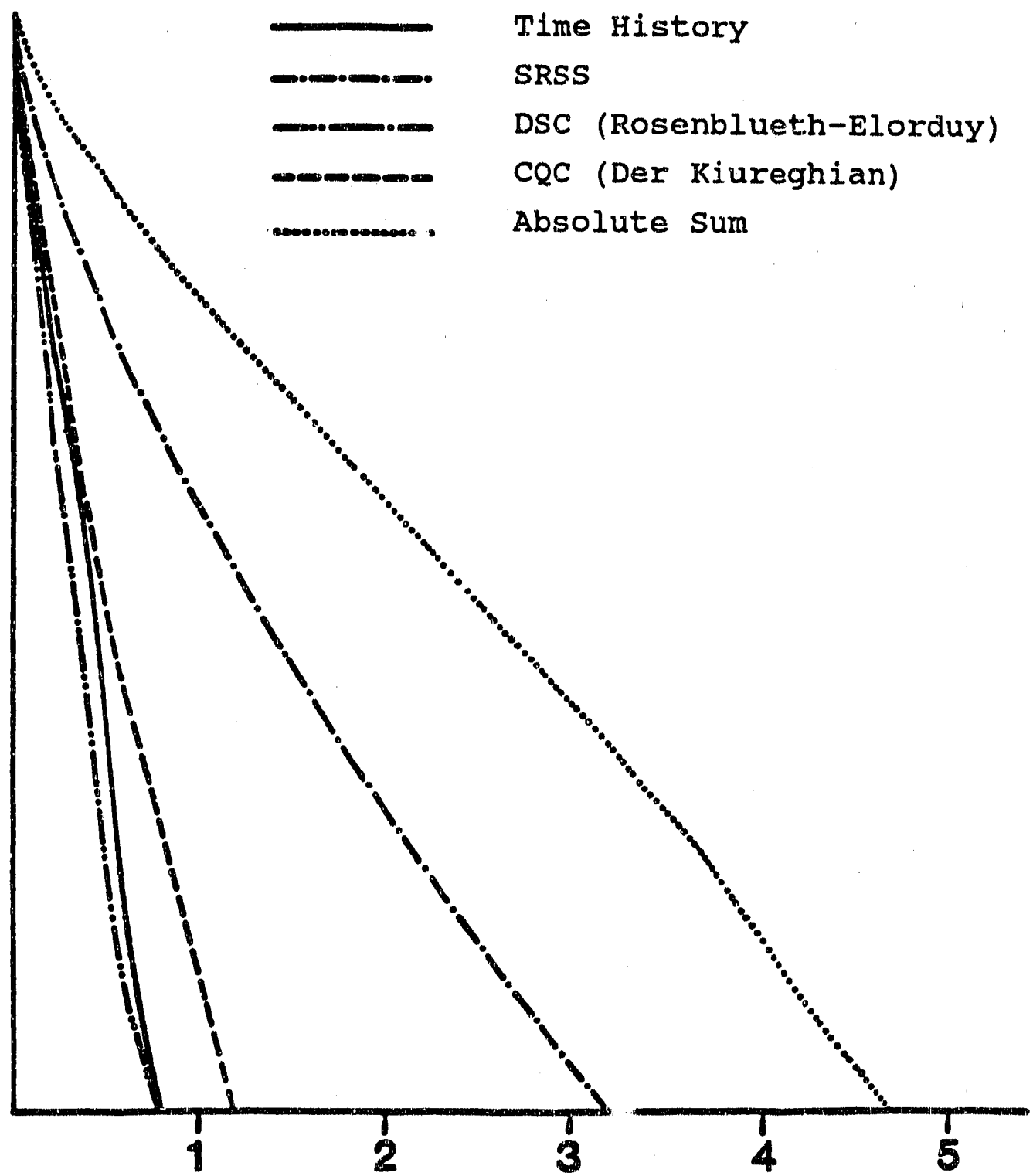

Story Overturning Moment $\left(\times 10^{6} \mathrm{kip}\right.$-inch $)$

Figure 3 Comparison of Modal. Combination Rules story Overturning Moments

$\mathrm{AA}-5$ 
Alternate Modal Combination Methods in Response Spectrum Analysis

5. AUTHORIS

P. Bezler, J.R. Curreri, Y.K. Wang, A.K. Gupta

\begin{tabular}{|c|c|}
\hline DATE REPORT & UBLISHED \\
\hline NONTH & YEAA \\
\hline October & 1990 \\
\hline $\begin{array}{l}\text { 4. FINOR GPANT NUM } \\
\text { A-395: }\end{array}$ & \\
\hline 6. TYPE OF REPORT & " \\
\hline Technical & Report \\
\hline 7. PERIOD COVERED & \\
\hline $\begin{array}{l}\text { October } 1 \\
\text { September }\end{array}$ & $\begin{array}{r}1988- \\
30,199\end{array}$ \\
\hline
\end{tabular}

8. PERFORMING ORGANIZA ION - NAME AND ADDRESS (II NRC. provide Division, Orlice or Region, U.S. Nuclear Regularory Cuminission, and mailing address; il contractor, provide name ond mailing address,

Brookhaven National Laboratory

Upton, NY 11973

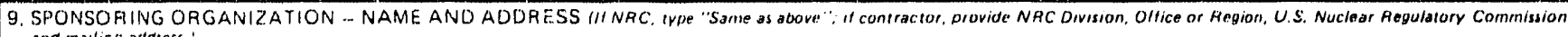

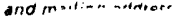

Division of Engineering

Office of Nuclear Regulatory Research

U.S. Nuclear Regulatory Commission

Washington. $D C \quad 20555$

10. SUPPLEMENTARY NOTES

11. ABSTRACT 1200 vooros or lessi

This technical report was prepared to document the results of an investigation of alternate methods to combine between modal response components when using the response spectrum method of analysis. These methods axe mathematically based to properly account for the combination between rigid and flexible modal responses as well as closely spaced modes. The methods are those advanced by Gupta, Hadjian and LindleyYow to address rigid response modes and the Double sum Combination (DSC) method and the Complete Quadratic combination (CQC) method to account for closely spaced modes. A direct comparison between these methods as well as the sRSS procedure is made by using them to predict the response of six piping systems. The results provided by each method are compared to the corresponding time history estimates of results as well as to each other. The degree of conservatisin associated with each method is characterized.

Nuclear Power Plants -- Pipes; Pipes -- Seismic Effects; Mathematical Models; Reactor Safety; Pipes -- Response Functions;

Reactor Components - - Seismic Effects; Frequency Analysis; 

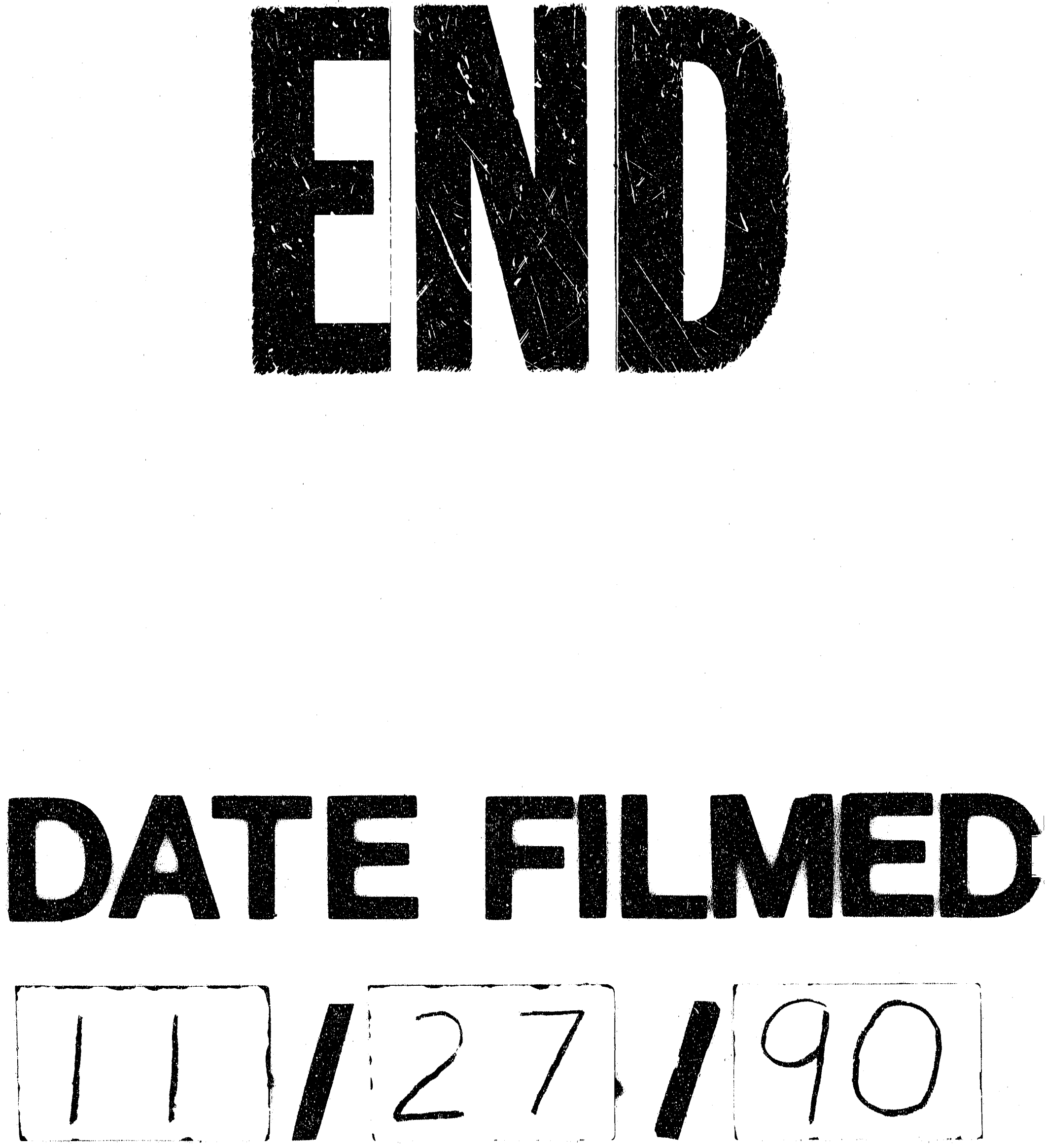
